University of Massachusetts Amherst

ScholarWorks@UMass Amherst

Doctoral Dissertations 1896 - February 2014

$1-1-1993$

\title{
The morphology of liquid crystalline polymers and their blends/
}

Kevin R. Schaffer

University of Massachusetts Amherst

Follow this and additional works at: https://scholarworks.umass.edu/dissertations_1

\section{Recommended Citation}

Schaffer, Kevin R., "The morphology of liquid crystalline polymers and their blends/" (1993). Doctoral Dissertations 1896 - February 2014. 815.

https://doi.org/10.7275/vfb6-zm68 https://scholarworks.umass.edu/dissertations_1/815

This Open Access Dissertation is brought to you for free and open access by ScholarWorks@UMass Amherst. It has been accepted for inclusion in Doctoral Dissertations 1896 - February 2014 by an authorized administrator of ScholarWorks@UMass Amherst. For more information, please contact scholarworks@library.umass.edu. 


\section{THE MORPHOLOGY OF LIQUID CRYSTALLINE POLYMERS AND THEIR BLENDS}

A Dissertation Presented

by

KEVIN R. SCHAFFER

Submitted to the Graduate School of the University of Massachusetts in partial fulfillment of the requirements for the degree of

DOCTOR OF PHILOSOPHY

May 1993

Polymer Science and Engineering 
CCopyright by KEVIN ROMAN SCHAFFER, 1993

All Rights Reserved 


\section{THE MORPHOLOGY OF LIQUID CRYSTALLINE POLYMERS AND THEIR BLENDS}

A Dissertation Presented

by

KEVIN R. SCHAFFER

Approved as to style and content by:
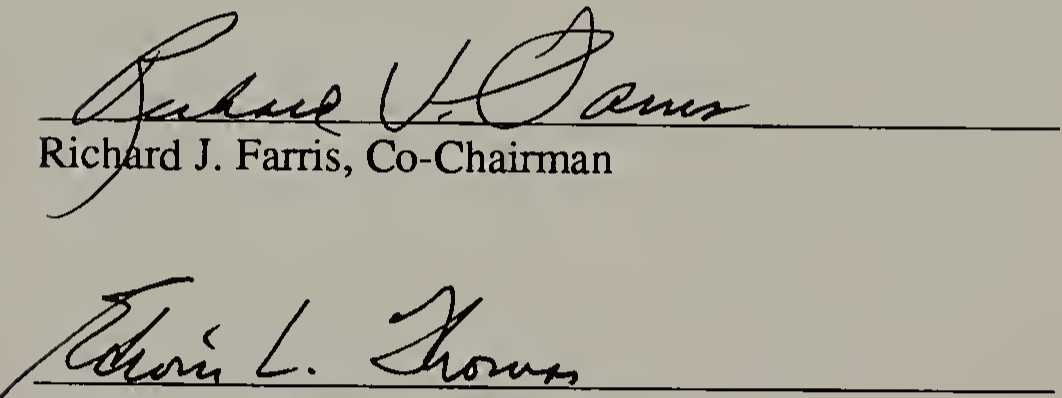

Edwin L. Thomas, Co-Chairman

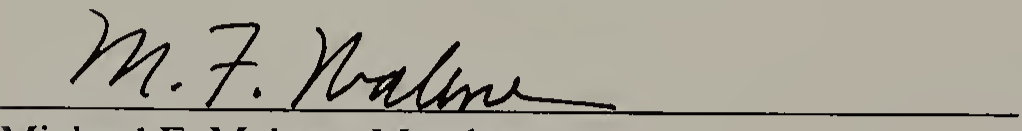

Michael F. Malone, Member

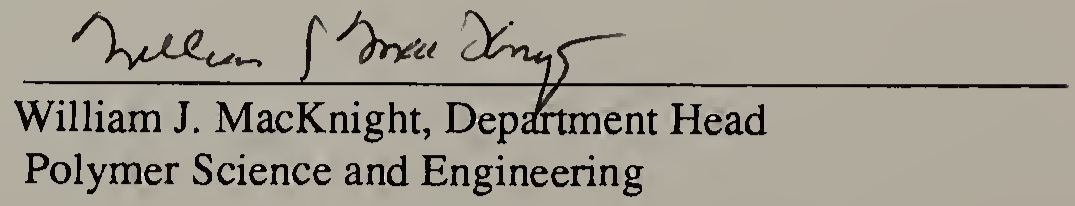


Dedicated to

Emma Schaffer 1898-1992

Albert H. Schaffer 1929-1976

LeRoy I. Braddock, Ph. D. 1925-1971 


\section{ACKNOWLEDGMENTS}

I would like to acknowledge my co-advisors, Professors Richard Farris and Edwin Thomas, for their guidance over these past few years. Their respective expertise has given me an example which I may emulate. I also feel a great pride in being associated with the Polymer and Science and Engineering Department; I cannot express enough my admiration for the students that I humbly call my colleagues. Especially, I wish to recognize the members of the groups of my advisors; I have never met a group of more dedicated, enthusiastic, intelligent and hard working persons.

I wish to express my appreciation to my wife, Mary, for without her love and support through discouraging times I would not be in the position to write these acknowledgments. Extreme thanks to my mother, Frieda, and my siblings Rich, Mark, and Cindy and their families who have given me support and understanding throughout my education. 


\section{ABSTRACT \\ THE MORPHOLOGY OF LIQUID CRYSTALLINE POLYMERS AND THEIR BLENDS \\ MAY 1993 \\ KEVIN ROMAN SCHAFFER, \\ B.S., NORTH CAROLINA STATE UNIVERSITY \\ M.S., NORTH CAROLINA STATE UNIVERSITY \\ M.S., UNIVERSITY OF MASSACHUSETTS \\ Ph. D., UNIVERSITY OF MASSACHUSETTS \\ Directed by}

Professors Richard J. Farris and Edwin L. Thomas

Nematic liquid crystals polymers (LCPs) encompass a large class of macromolecules exhibiting orientational order when in the melt or solution state. The intrinsic local orientation of these materials yields processing advantages due to lower viscosities than related isotropic (flexible coil) polymers of comparable molecular weight. Highly directional moduli and strengths in the solid state are further benefits of the ease of obtaining high orientations during processing in the melt or solution.

Blending and composite fabrication with other materials are the practical methods that must be developed to use LCPs for structural applications. A nematic liquid crystal polymer such as poly(p-phenylene benzobisthiazole) (PBZT) is a rigid rod macromolecule with its length being essentially the mesogenic unit. Such rigid rod polymers are usually lyotropic liquid crystals and are infusible in the 
pure state so that any fabrication with other materials must be done in the solid or solution state.

Thermotropic liquid crystalline polymers have molecular architecture usually consisting of shorter mesogenic units connected with flexible spacers that allow the mobility needed for the existence of flow at higher temperatures. The melt state allows the possibility of melt blending with other polymers using the liquid crystal as a processing aid or a reinforcement that forms in-situ.

The purpose of this thesis is to characterize the morphology on various length scales of polymer composite materials obtained by these disparate methods of fabrication. The principle techniques utilized are optical, transmission electron, scanning electron microscopy, and X-ray diffraction.

This work consists of an introductory and three subsequent chapters. Chapter 2 describes the morphology of composite films fabricated by attempting to infiltrate isotropic materials (glass or epoxy) into an existing nano-scale fibrillar network that naturally forms when PBZT is coagulated. The PBZT film that was formed by extrusion through a counter-rotating die.

In Chapter 3, the morphology of fibers formed from spinning dopes composed of mechanically mixed PBZT and flexible polymer solutions is discussed. In Chapter 4 the morphology of a melt blended thermotropic liquid crystal polymer is compared with mechanical data. 


\section{TABLE OF CONTENTS}

Page

ACKNOWLEDGMENTS .

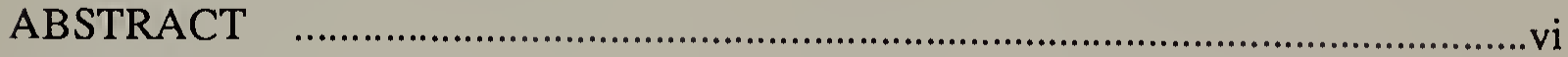

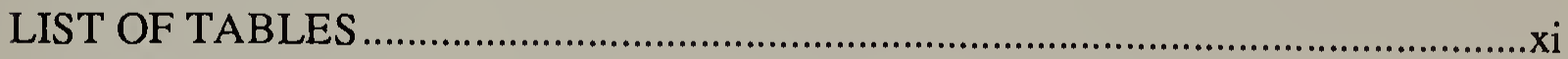

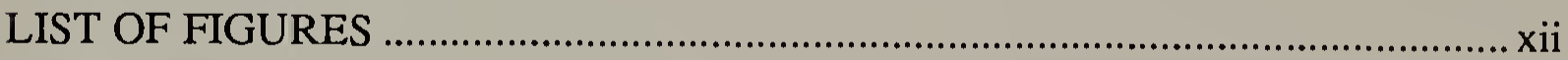

Chapter

1. OVERVIEW AND LIQUID CRYSTAL THEORY ….....................................

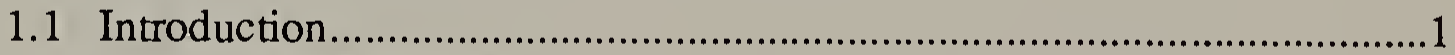

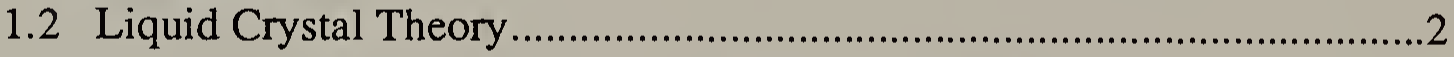

1.2.1 Anisotropic Interaction and Hard Rod Theories ................................

1.2.2 Elastic Continuum Theory of Nematics. ...........................................9

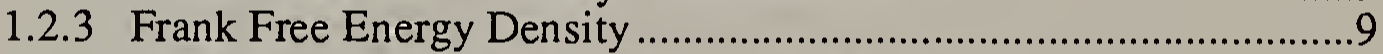

1.2.4 Surface terms in Frank continuum theory .................................12

1.2.5 The Nematic Interface ................................................................... 14

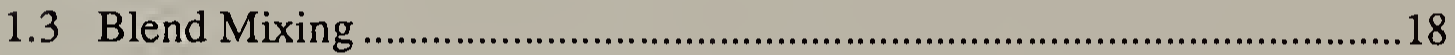

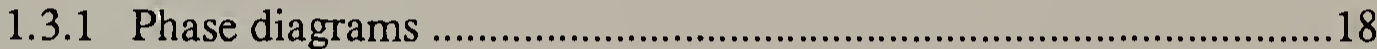

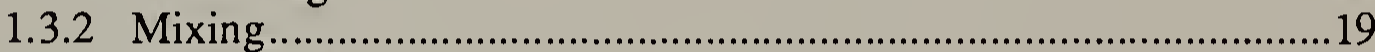

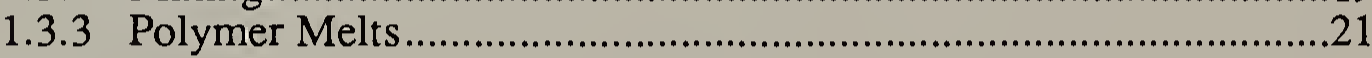

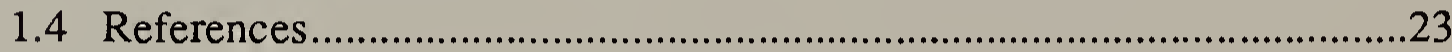

2. INFILTRATION OF PBZT FILMS WITH SOL-GEL GLASS ….......................27

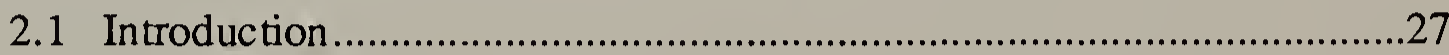

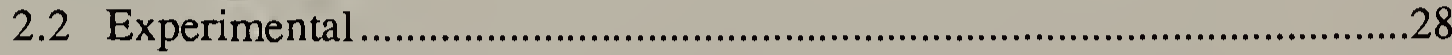

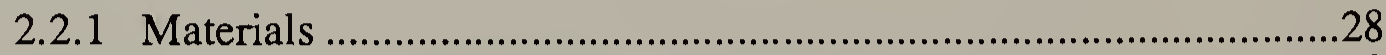

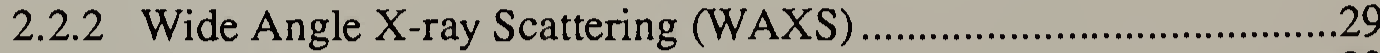

2.2.3 Microscopy ………......................................................................

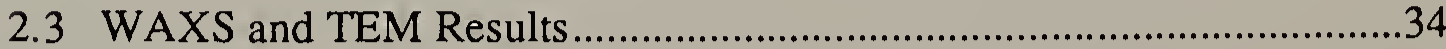

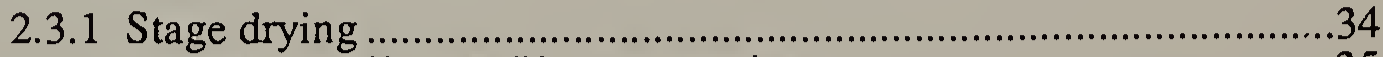

2.3.2 Annealing effect on X-ray scattering ...............................................

2.3.3 Orientation Distribution Estimation …………..............................36

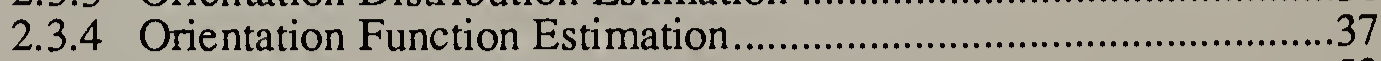

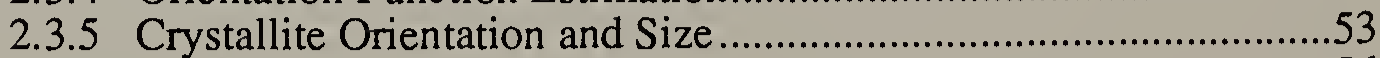

2.3.6 TEM Images ................................................................................ 


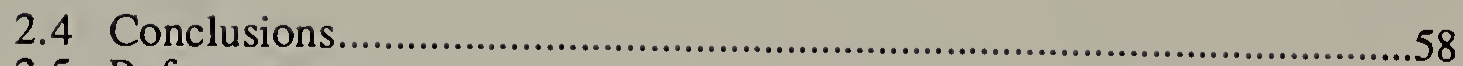

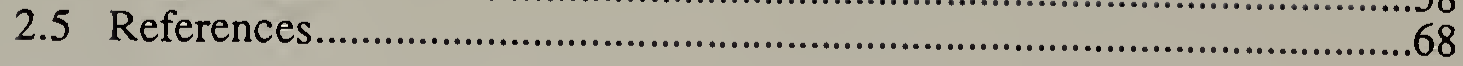

3. LIQUID CRYSTAL POLYMER FIBER BLENDS BY SOLUTION BLENDING

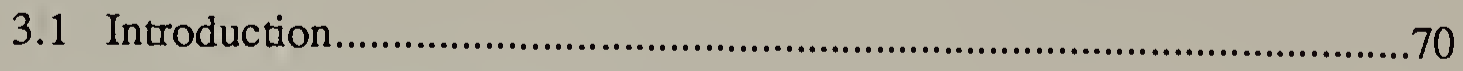

3.1.1 Liquid crystal solution blends ..................................................71

3.1.2 Rheological behavior of multi-phase systems ................................ 71

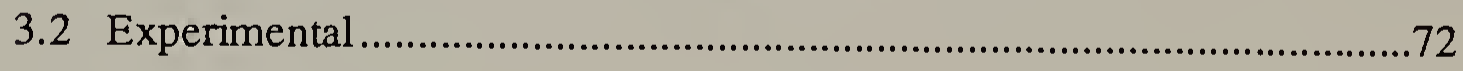

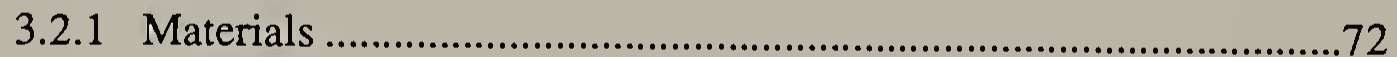

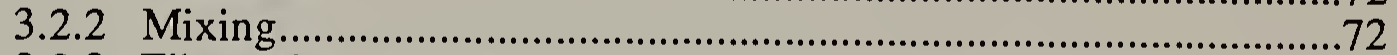

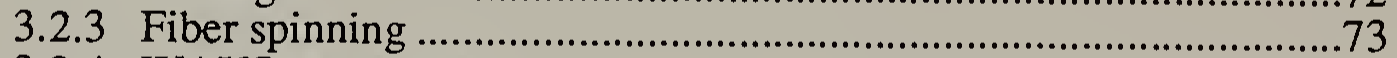

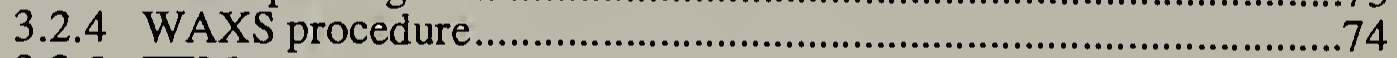

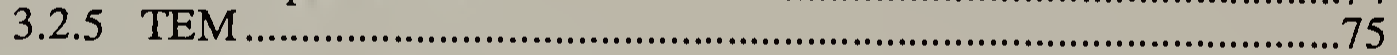

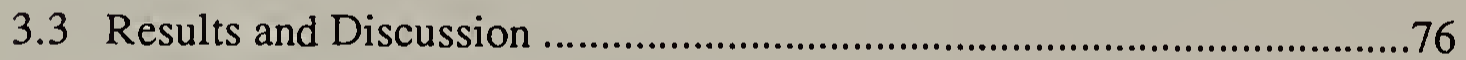

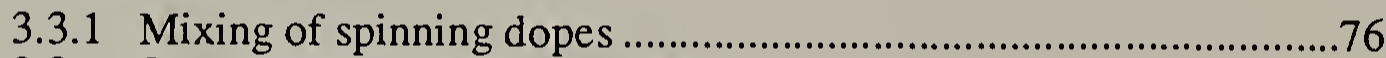

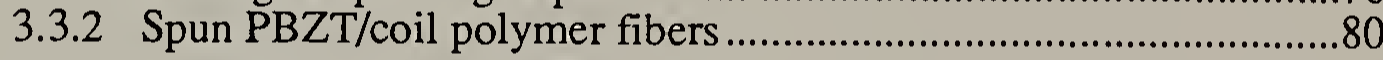

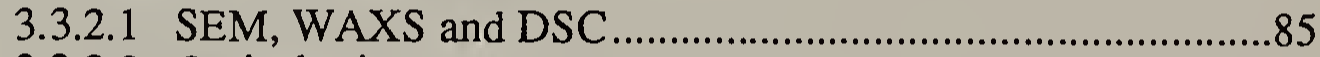

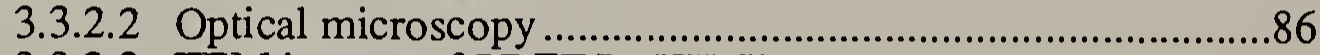

3.3.2.3 TEM images of PBZT/PEKK fibers ......................................87

3.3.3 Consolidating the composite fibers .............................................101

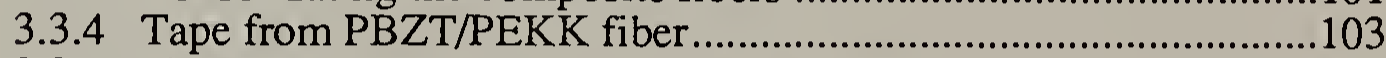

3.3.5 Morphology of PBZT/PEKK Tensile Bar ...................................105

3.3.6 PBZT/Polyamide composites ........................................................107

3.4 Composite properties ..........................................................................107

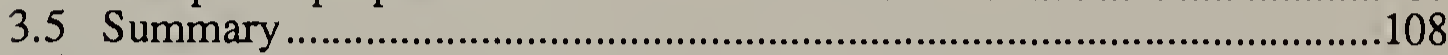

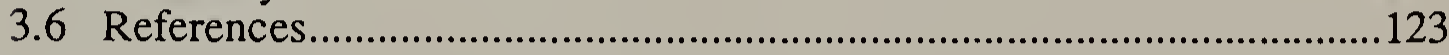

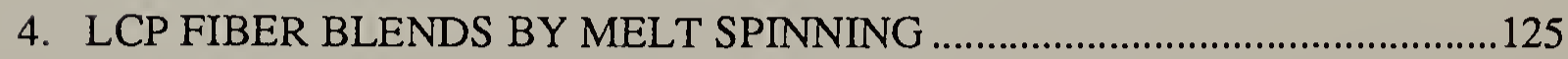

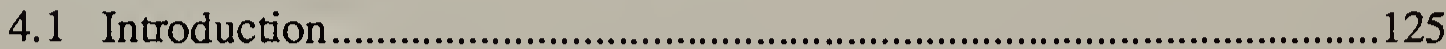

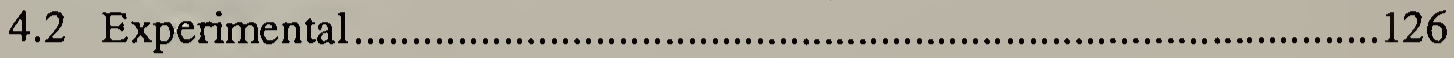

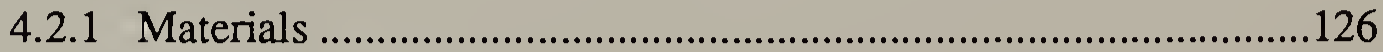

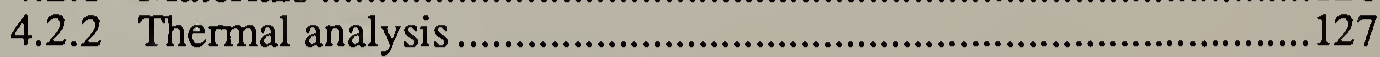

4.2.3 Fiber spinning and properties ......................................................127

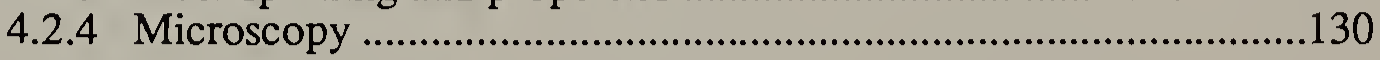

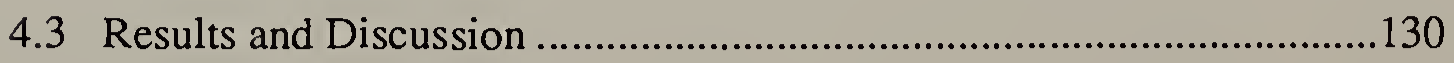

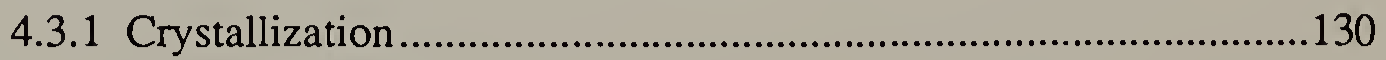


4.3.2 Tensile properties....................................................................133

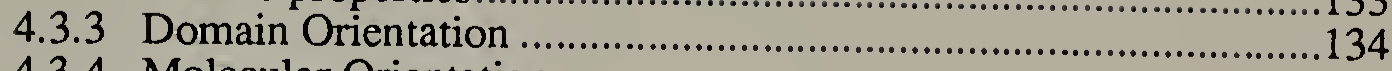

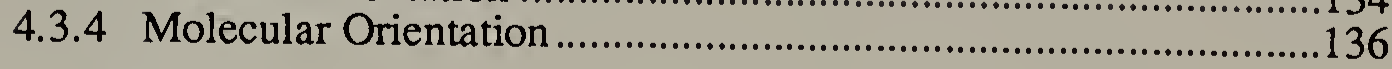

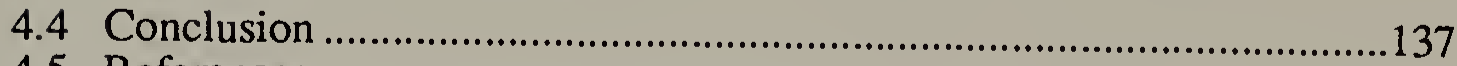

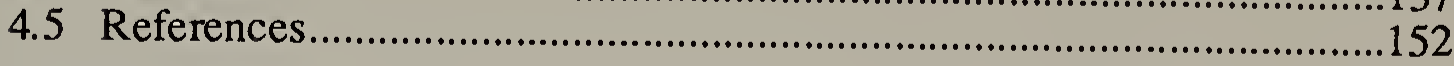

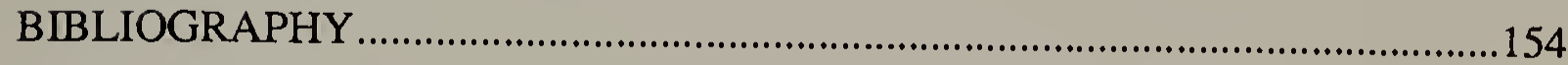




\section{LIST OF TABLES}

Table

Page

2.1 Description of PBZT/ Glass Samples ............................................... 30

2.2 Wide Angle X-ray Diffraction Data .................................................. 41

2.3 Indexing of Hot Pressed PBZT/Lead Borate Composite Film .................... 42

2.4 Orientation Function Values for Cylindrical Symmetry ........................... 51

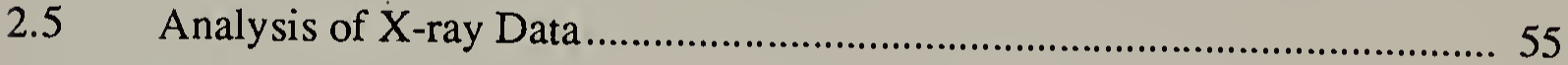

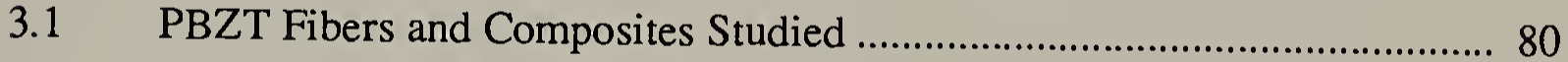

3.2 PBZT/PEKK Composite Fiber (PBZT Reflections Only) ......................... 92

3.3 PBZT/PEKK Composite Fiber (PEKK Reflections Only)......................... 92

3.4 PEKK d-spacings (Melt Drawn Fiber) ................................................. 93

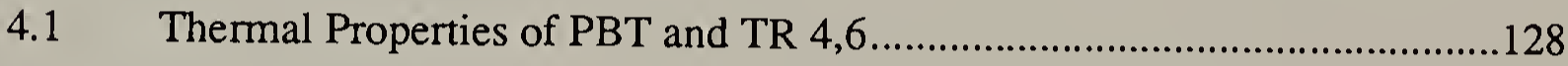

4.2 Effect of LCP Content and Annealing .................................................132 


\section{LIST OF FIGURES}

Figure

Page

1.1 (A) A rodlike particle oriented at angle $\psi$ to the preferred axis of the domain. (B) The representation of the particle as y sequences or submolecule, each parallel to the domain axis. Explicitly, $\psi$ is the angle of inclination to the $x$ axis and $x$ is the length of the molecule

1.2 The three basic deformations of a uniaxial nematic (A) splay, (B) twist, and $(\mathrm{C})$ bend

2.1 Schematic demonstrating (A) biaxial film production (B) the natural curl in film and $(C)$ the coordinate system of $X$-ray experiments

2.2 (A) SEM image of a cleaved PBZT biaxial film showing the two main layers into which the film readily divides. The fibrils tend to orient in two different directions in the two film halves. (B) SEM image of similar film in which the fracture surface indicates a twist in the fibril orientation through the film thickness

2.3 Schematic of infiltration procedure

2.4 PBZT film $X$-ray diffraction patterns with the $X$-ray beam incident from the X-direction. (A) Control (No glass), stage dried at $300^{\circ} \mathrm{C}$. (B) Silicate, Stage dried at $350^{\circ} \mathrm{C}$. (C) Silicate, Hot Pressed at $500^{\circ} \mathrm{C}$. (D) Sodium Borosilicate Stage dried at $350^{\circ} \mathrm{C}$. (E) Sodium Borosilicate Hot Pressed at $500^{\circ} \mathrm{C}$. (G) Lead Borate Stage at $300^{\circ} \mathrm{C}$. (H)Lead Borate, Heat treated at $300^{\circ} \mathrm{C}$

2.5 X-ray diffraction patterns with beam incident from the $\mathrm{x}$-direction of PBZT film (A) that splits into two layers; an outer layer (B) and an inner layer (C). $\phi$ is the azimuthal angle with the $\mathrm{z}$ axis shown in Figure 2.1

2.6 The schematic demonstrates the method of obtaining integrated intensity versus azimuthal angle plots from the X-ray diffraction patterns 
2.7 (A.) A trace of relative integrated intensity verses the azimuthal angle, $\phi$, for the $X$-ray patterns in Figure 2.5. The intensities of the two film halves were weighted so their sum will yield the trace for the full film. (B.) A comparison of the sum from $2.7 \mathrm{~A}$ and the relative intensity versus azimuthal angle, $\phi$, for the whole PBZT film

2.8 X-ray diffraction patterns from a heat treated PBZT/borosilicate glass composite film (A) split into two layers; an outer layer (B) and an inner layer $(C)$. $\phi$ is the azimuthal angle with the $z$ axis and the molecular orientation.

2.9 (A.) A trace of relative integrated intensity verses the azimuthal angle, $\phi$, for the X-ray patterns in Figure 2.8. The intensities of the two film halves were weighted so their sum will yield the trace for the full film. (B.) A comparison of the sum from 2.9A and the relative intensity versus azimuthal angle, $\phi$, for the whole PBZT film.

2.10 A schematic showing the relationship between $\phi, \beta$ and $\mathrm{r}(2 \theta)$ [Alexander, 1969]

$2.11 \mathrm{X}$-ray diffraction patterns from folded biaxially oriented $\mathrm{PBZT}$ /borosilicate film. The X-ray beam is incident from the $y$ direction. The [010] reflection has a much higher intensity due to the crystallite orientation within the $y-z$ plane

2.12 A trace of relative integrated intensity verses the azimuthal angle, $\phi$, for (A) a folded PBZT film and (B) a folded PBZT/borosilicate in which the X-ray beam is incident in the y direction (see Figure 2.1C) 50

2.13 A suggested model for the through thickness orientation distribution based upon SEM and X-ray diffraction evidence.

2.14 The method of acquiring of the relative azimuthally integrated intensity versus scattering angle, $2 \theta$, from $X$-ray diffraction patterns

2.15 A comparison of azimuthally integrated intensity versus scattering angle for PBZT films with the X-ray incident from (A) the $x$ direction and (B) the y direction. Crystallite size estimates are given in Table $2.5 \ldots . .55$ 
2.16 The schematic demonstrates the orientation of the film samples for microtoming

2.17 TEM bright field images for thin sections of (A) PBZT control film and PBZT/lead borate glass composite film that is stage dried (B) and heat pressed $(C)$ showing the increase in the number of dark lead borate domains. The respective dark field images, C, D, and $\mathrm{E}$ show the high crystallinity of some of the lead borate particles

2.18 TEM bright field images of thin sections of an epoxy impregnated

PBZT film and a PBZT/lead borate glass composite film, comparing the size of the voids of the PBZT microfibrillar network and the size of the lead borate particles

2.19 TEM bright field (A) and dark field (B) images of a thin section of a $\mathrm{PBZT}$ /borosilicate glass composite film in which the borosilicate glass appears to be in sheet form. In (A) the location of the borosilicate sheets are marked with "s"

2.20 Bar graphs of tensile properties for biaxially balanced films with various types of glasses and glass contents

3.1 The materials used in this study 74

3.2 A schematic of a fiber spinning apparatus 75

3.3 Orientational of views for TEM images 76

3.4 A 69/31 PBZT/polyamide (13\% solids) spinning mixture slightly sheared between glass slides in the indicated direction without (A) and with (B) cross polarizers parallel to the image edges. (C) and (D) are images (with and without cross polarizers, respectively, of neat PBZT spinning dope sheared between glass slides that show no domain structure)

3.5 Optical micrographs of 69/31 PBZT/polyamide (11\% solids) spinning dope samples between glass slides after mixing for (A) 2.5 hours, (B) 3 hours, (C) 4.7 hours, (D) 17.5 hours The sizes of the polyamide rich regions appear to attain an average steady state size of approximately 2 microns 
3.6 Reflective optical micrographs of sectioning block showing (A) a cross section and (B) a longitudinal section of a fiber composed of $69 / 31 \mathrm{PBZT} /$ polyamide fiber spun at spin stretch ratio of 1

3.7 TEM images showing (A) bright and (B) dark field images of cross section of a fiber composed of 69/31 PBZT/polyamide fiber spun at spin stretch ratio of 1

3.8 TEM images showing (A) bright and (B) dark field images longitudinal sections of fibers composed of $69 / 31$ $\mathrm{PBZT} /$ polyamide fiber spun at spin stretch ratio of 1

3.9 Optical micrographs (reflective mode) of cross sections of PBZT/polyamide fibers spun with the following draw ratios: (A) 1 and (B) 4 show the change in diameter, internal structure, and the noncircular cross sections of the fibers

3.10 Scanning electron micrographs of PBZT/PEKK fibers $(A)$ before and

(B) after ultrasonification indicating that a coating is on the fibers

3.11 X-ray diffraction patterns of as-spun (A) and heat treated (B) fibers indicate the presence of crystallinity. The patterns are indexed in Tables 3.2 and 3.3. The schematic (C) shows the indices of the most prominent reflections

3.12 X-ray diffraction patterns of PEKK as received film (A) and fibers (B) pulled from the melt at $300 \mathrm{C}$. The reflections of (B) are indexed in Table 3.4. A schematic (C) of the reflections of (B)

3.13 Differential scanning calorimetry scan of the as received PEKK polymer sample. The material crystallizes readily at the $20^{\circ}$ $\mathrm{C} / \mathrm{min}$ heating rate. The similar area under the crystallizing and melting peaks indicates limited crystallinity in the as received PEKK sample

3.14 Optical micrographs of sectioning block showing (A) cross and (B) longitudinal sections of fibers composed of $60 / 40$ PBZT/PEKK (AS-10). Optical micrographs of the PBZT/PEKK fibers after heat treatment (HT-10) showing (C) cross and (D) longitudinal sections. One observes structure on the micron scale. 
3.15 A TEM bright field image (A) shows a cross section of an as spun fiber composed of 60/40 PBZT/PEKK. The epoxy of the sectioning block at the fiber surface is labeled with an $\mathrm{E}$. The area in the rectangle is shown at higher magnification in Figure 16

3.16 A TEM bright field image of a cross section of an as-spun fiber composed of $60 / 40$ PBZT/PEKK at higher magnification in which the fine phase structure suggests a cross section of the microfibrillar network observed by Cohen

3.17 A TEM bright field image of a near longitudinal section of a heat treated fiber composed of 60/40 PBZT/PEKK, shows elongated PEKK domains. An E marks the epoxy at the surface of the fiber. The bright areas in the dark field image (B) indicate diffracting PBZT from equatorial reflections

3.18 A TEM of a cross section of a neat PBZT film showing no structure other than approximately 10 nanometer diameter fibrils

3.19 Schematic of composite formation in composites 102

3.20 An optical micrograph (reflective) of a microtome sectioning block showing a cross section of a tape fabricated by pressing $60 / 40$ PBZT/PEKK fibers. The image shows the outlines of individual fibers and their internal structure. The fiber cross sections deform to fill the space of the cross section

3.21 A series of TEM images of a thin cross section of a tape fabricated by pressing 60/40 PBZT/PEKK fibers. A bright field image (A), and dark field images using the PBZT equatorial [100] and [010] reflections (B) and diffuse scatter due to PEKK (C) showing the internal structure of the individual fibers

3.22 An optical micrograph (reflective) of microtome sectioning block showing a cross section (A) and a longitudinal section (B) of a composite fabricated from the PBZT/PEKK fiber tape (MB-10)

3.23 A bright field image (A), and dark field images, (B and $C$ ) using the PBZT equatorial [100] and [010] reflections (B) and diffuse scatter (C) due to PEKK of a PBZT/PEKK composite cross section of sample TA-10 
3.24 A bright field image (A), and dark field images using the PBZT equatorial [100] and [010] reflections (B) of a near longitudinal section from PBZT/PEKK fiber composite MB-10

3.25 An optical micrograph (reflective) of microtome sectioning block showing cross sections of (A) as-spun PBZT/polyamide fiber and (B) acid treated fiber and (C) longitudinal section of the acid treated fiber

3.26 An optical micrograph (reflective) of microtome sectioning block showing a cross section of a composite bar fabricated from a PBZT/polyamide acid treated fiber. The outlines of the individual fibers are not as distinct

3.27 A bright field image (A), and dark field image (B) using the PBZT equatorial [100] and [010] reflections of a cross section from a composite fabricated from acid treated PBZT/polyamide fibers

3.28 (A) Specific tensile, (B) flex moduli, (C) tensile strength, (D) flexural strength, and (E) compressive strength data [Perusich, 1990]

3.29 A schematic composite fabricated from an immiscible PBZT/coil polymer blend fiber.

4.1 Structures of (A) poly (butylene terephthalate) and (B) the random TR4,6 (1/1) liquid crystal polymer used for blending

4.2 Schematics of spinning fiber blend (A) and post hot draw (B)

4.3 DSC scan of the TR4,6 random copolymer

4.4 DSC scans of TR4,6/PBT blend specimens with varying LCP content

4.5 DSC scans of the $80 / 20 \mathrm{TR} 4,6 / \mathrm{PBT}$ after various times at $255^{\circ} \mathrm{C}$

4.6 Graph showing the effect of spin stretch ratio upon (A) the modulus and $(B)$ the breaking strength of as-spun fibers 
4.7 Graph showing the effect of liquid crystal content upon (A) the modulus and (B) the breaking strength of as-spun and post hot drawn fibers

4.8 Optical micrographs of TR4,6/PBT blends with crossed polarizers parallel to the image edges

4.9 Thin section of the as-spun and post drawn 20/80 TR 4,6/PBT fiber. (A) as-spun, no polarizers, (B) post hot drawn, no polarizers, (C) as-spun, cross polarizers, (D) post hot drawn, cross polarizers, (E) as-spun, cross polarizers, $240^{\circ} \mathrm{C}(\mathrm{F})$ post hot drawn, cross polarizers, $240^{\circ} \mathrm{C}$. The polarizers are parallel to the image edges

4.10 An SEM of a 20/80 TR 4,6/PBT as-spun fiber shows a transverse cryogenic fracture displaying the particulate nature of the LCP inclusions

4.11 An SEM of a 20/80 TR 4,6/PBT hot drawn fiber showing an (A) transverse and (B) longitudinal cryogenic fracture displaying a fibrillar nature

4.12 TEM images of a thin section of the hot drawn PBT fiber in bright field $(\mathrm{A})$ and in dark field (B) using equatorial reflections and (C) the electron diffraction pattern

4.13 TEM images of a thin section of the as-spun 20/80 TR 4,6/PBT fiber in bright field (A) and in dark field (B) using equatorial reflections and $(\mathrm{C})$ the electron diffraction pattern

4.14 TEM images of a thin section of the post hot drawn (D.R.=5) 20/80 TR 4,6/PBT fiber with bright field (A) and in dark field (B) using equatorial reflections and $(C)$ the electron diffraction pattern

4.15 TEM images show a thin section of the post hot drawn 20/80 TR 4,6/PBT fiber with bright field conditions (A) and in dark field conditions (B) using equatorial reflections and (C) the electron diffraction pattern. The section was heated to $240^{\circ} \mathrm{C}$ on a hot stage then quenched prior to viewing. The electron diffraction pattern is indexed in (D) 


\section{CHAPTER 1}

\section{OVERVIEW AND LIQUID CRYSTAL THEORY}

\subsection{Introduction}

Nematic liquid crystals polymers (LCPs) encompass a large class of macromolecules exhibiting orientational order when in the melt or solution state. The intrinsic local orientation of these materials yields processing advantages due to lower viscosities than related isotropic (flexible coil) polymers of comparable molecular weight. Highly directional moduli and strengths in the solid state are further benefits of the ease of obtaining high orientations during processing in the melt or solution.

Blending and composite fabrication with other materials are the practical methods that must be developed to use LCPs for structural applications. A nematic liquid crystal polymer such as poly(p-phenylene benzobisthiazole) (PBZT) is a rigid rod macromolecule with its length being essentially the mesogenic unit. Such rigid rod polymers are usually lyotropic liquid crystals and are infusible in the pure state so that any fabrication with other materials must be done in the solid or solution state. Rather than a limitation, such restrictions may lead to both novel composites and methods to obtain them.

Thermotropic liquid crystalline polymers have molecular architecture usually consisting of shorter mesogenic units connected with flexible spacers that allow the mobility needed for the existence of flow at higher temperatures. The melt state allows the possibility of melt blending with other polymers using the liquid crystal as a processing aid or a reinforcement that forms in-situ.

The purpose of this thesis is to characterize the morphology on various length scales of polymer composite materials obtained by these disparate methods of 
fabrication. The principle techniques utilized are optical, transmission electron, scanning electron microscopy, and X-ray diffraction.

This work consists of an introductory and three subsequent chapters. In the remainder of this chapter, a general theoretical background of liquid crystalline materials is presented.

Chapter 2 describes the morphology of composite films fabricated by attempting to infiltrate isotropic materials (glass or epoxy) into an existing nano-scale fibrillar network that naturally forms when PBZT is coagulated. The PBZT film that was formed by extrusion through a counter-rotating die resulting in a biaxial orientation through the film thickness is also characterized.

In Chapter 3, the morphology of fibers formed from spinning dopes composed of mechanically mixed PBZT and flexible polymer solutions is discussed. The PBZT/flexible polymer fibers exhibit a two domain structure, ranging from microns to nanometers in scale, which allows the fiber to be highly deformable in cross-section. In Chapter 4 the morphology of a melt blended thermotropic liquid crystal polymer is compared with mechanical data.

\subsection{Liquid Crystal Theory}

The first section will review the theories for the nematic isotropic transitions in LCPs and nematic liquid crystals. The order parameter is the characterization of the orientation function of the anisotropic molecules. Changes in the equilibrium orientation function with concentration, temperature, or molecular geometry cause the phase transitions from the isotropic to nematic phase. The second and third sections review continuum descriptions of long range fluctuations of the average local molecular direction or director and the associated free energy due to distortions. The last section reviews literature on blend mixing. 


\subsubsection{Anisotropic Interaction and Hard Rod Theories}

The derivation of molecular field theories depends upon the assumption of the nature of the intermolecular potential in the system. For instance, in the Maier-Saupe Theory the initial assumption is that nematic order results from anisotropic intermolecular attractions [Cotter, 1983]. The intermolecular potential mean torque on a molecule at angle $\beta$ with the laboratory axis, $U(\beta)$, is defined in terms of the singlet orientation distribution function for the simplified case of uniaxial molecular symmetry, $f(\beta)=\exp \{U(\beta) / k T\} / Z$, with $Z$ being a normalization term. The mean torque is assumed to be the effect of the anisotropic dispersion forces that arise from the rod character of the molecule [Luckhurst, 1983b]

The function $f(\beta)$ describes the statistical distribution of molecular orientation due to thermal motion. The distribution is characterized by the coefficients obtained by expansion in terms of spherical harmonics called the molecular order parameters [Clark, 1985]. The derivation of the distribution function from specific molecular interactions to obtain the order parameters is a complicated task and requires averaging assumptions to reduce the amount of calculation. The crux of the methods is to determine the distribution function in which the chemical potential of the system is a minimum [de Gennes, 1974a].

In "hard rod" theories the nematic order is due to excluded volume effects resulting from repulsive forces that act at very short range. The basic model used in these theories is infinitely impenetrable rod-like objects [Cotter, 1983]. A combination of the Maier-Saupe and hard rod theories leads to Van der Waals type theories where the molecules are modeled by hard cores along with mean field averaged anisotropic intermolecular attractions [Cotter, 1983]. In the Onsager method excluded volume is accessed for a pair of rod like particles in a gas as a function of their relative orientations. Second virial coefficients can be determined by averaging over all orientations for the 
isotropic case. By using the condition of minimum free energy, the equilibrium distribution of orientations for an anisotropic phase can be determined [Flory, 1956]. The Onsager treatment leads to qualitative predictions of the transition points and the final order parameter of the nematic state due to difficulty in expanding the excluded volume terms that apply to clusters of rod molecules greater than two. Consequently, the theory is restricted to concentrations where higher virial coefficients are neglected [Ballauff, 1989].

Flory and Ronca [1979a] developed a polymer solution theory using the hard rod model similar to Onsager but utilizing a lattice to simplify calculation of the configurational partition functions [Flory and Ronca, 1979a]. The theory can incorporate Maier-Saupe attractive interactions as intermolecular energy perturbations. The advantage of using a lattice is that one can use a priori or mathematical probabilities to calculate the partition functions since one can assume random placement of the elements (polymer segments or submolecules) onto the lattice. Since a mean field is usually assumed, knowledge of the environment of each individual molecule is not required.

The essential step of the latter is to divide the rod molecules into a sequence of $y$ consecutive submolecules. In two dimensions a molecule which is at an angle $\psi$ to the orientation axis is divided into $\mathrm{y}=\mathrm{x} \sin \psi$ sequences each being $\mathrm{x} / \mathrm{y}$ in length [Flory, 1956]. Figure 1.1 shows the a graphical representation of the division of the rod molecules into submolecules to model disorientation with respect to a preferred axis.

The calculation of the configuration for a system of $n_{x}$ rods depends upon the evaluation of the number of situations, $v_{i+1}$, available to the $i+1$ th rod after $i$ particles are in the lattice. The probability that a succeeding site is vacant after the first segment of molecule $i+l$ is placed in the lattice is equivalent to the mole fraction of the vacant sites if the previous submolecules and vacant sites are randomly distributed [Ballauff, 1989]. 
The initial segment of the $y_{i+1}-1$ submolecules is given by the volume fraction of vacancies after placement of ix segments, $\left(\mathrm{n}_{0^{-}} \mathrm{ix}\right) / \mathrm{n}_{0}$. Since rod molecules are being placed a conditional probability is required that lattice sites parallel to the preferred molecular direction be vacant for placement of the remaining submolecules. The conditional probability is given by the ratio of vacant sites to the sum of vacant sites and the sites occupied by sub molecules which is true for random placement. The combination of all the factors yields the expectation for the placement of $\mathrm{x}$ segments of rod $i+1$ being arranged in $y_{i+1}$ submolecules:

$$
v_{\mathrm{i}+1}=\left(n_{0}-\mathrm{ix}\right)\left[\left(n_{0}-\mathrm{ix}\right) / n_{0}\right]{ }^{\left(\mathrm{y}_{\mathrm{i}+1}\right)-1}\left[\left(n_{0}-\mathrm{ix}\right) /\left(n_{0}-\mathrm{ix}+\sum_{\left.\mathrm{y}_{\mathrm{i}}\right)}\right]^{\mathrm{x}-\mathrm{y}_{\mathrm{i}+1}}\right.
$$

The partition function $\mathrm{Z}$ is assumed to split into a combinatorial part and in orientational part., $\mathrm{Z}=\mathrm{Z}_{\text {comb }} \mathrm{Z}_{\text {orient }}$ where,

$$
Z_{\text {comb }}=\left(1 / n_{\mathbf{x}} !\right) \prod_{v_{i} \cdot=}^{n_{x}}\left[\left(n_{1}+n_{x} y_{\text {avg }}\right) ! / n_{1} ! n_{x} !\right] n_{0} n_{x}\left(1-y_{\text {avg }}\right)
$$

The variables are $n_{1}$ and $n_{x}$ are the number of solvent and rod molecules respectively and

$$
\mathrm{y}_{\mathrm{avg}}=\sum_{\mathrm{n}_{\mathrm{xy}} \mathrm{y} / \mathrm{n}_{\mathrm{x}}}
$$

The term $n_{x y} / n_{x}$ is the orientational distribution specifying the fraction of the molecules with disorientation y. The orientational partition function is written in terms of the reciprocal of the orientation distribution

$$
\mathrm{Z}_{\text {orient }}=\prod_{\mathrm{y}}\left[\sigma_{\mathrm{x}} \omega_{\mathrm{y}}\left(\mathrm{n}_{\mathrm{x}} / \mathrm{n}_{\mathrm{xy}}\right)\right]^{\mathrm{n}_{\mathrm{x} y}}
$$

or

$$
\ln \mathrm{Z}_{\text {orient }}=\sum_{\mathrm{n}_{\mathrm{xy}}} \ln \left[\left(\mathrm{n}_{\mathrm{x}} / \mathrm{n}_{\mathrm{xy}}\right) \omega_{\mathrm{y}}\right]+\mathrm{n}_{\mathrm{x}} \ln \sigma_{\mathrm{x}}
$$

where $\omega_{y}$ represents the fractional range of solid angle for an interval of orientation which because of cylindrical symmetry is given by the expression

$$
\omega_{y}=\sin \Psi_{y}
$$


The adjustable parameter $\sigma_{\mathrm{x}}$ is chosen so that the statistical weight factor, $\sigma_{\mathrm{x}} \omega_{\mathrm{y}}$, is equal to unity. The free energy of a system of $n_{x}$ rigid rods immersed in a solvent containing $\mathrm{n}_{1}$ molecules is written by combining the orientational and combinatorial terms:

$$
\begin{aligned}
& -\ln \mathrm{Z}=\mathrm{n}_{1} \ln v_{1}+\mathrm{n}_{\mathrm{x}} \ln \left(v_{\mathrm{x}} / \mathrm{x}\right)-\left(\mathrm{n}_{1}+\mathrm{n}_{\mathrm{x}} \mathrm{y}\right) \ln \left[1-v_{\mathrm{x}}\left(1-\mathrm{y}_{\mathrm{avg}} / \mathrm{x}\right)\right)+\mathrm{n}_{\mathrm{x}}\left(\mathrm{y}_{\mathrm{avg}}-1\right) \\
& +\mathrm{n}_{\mathrm{x}} \sum_{\mathrm{n}_{\mathrm{xy}} / \mathrm{n}_{\mathrm{x}} \ln \left(\mathrm{n}_{\mathrm{xy}} /\left(\mathrm{n}_{\mathrm{x}} \omega_{\mathrm{y}}\right)-\mathrm{n}_{\mathrm{x}} \ln \sigma\right.}
\end{aligned}
$$

where $v_{1}$ and $v_{\mathrm{x}}$ are volume fractions of the respective solvent and polymer components. The system described is at equilibrium with the anisotropic phase measured by the disorder index y. At orientational equilibrium

$$
\left(d \ln \mathrm{Z} / d\left(\mathrm{n}_{\mathrm{xy}} / \mathrm{n}_{\mathrm{x}}\right)\right)=0
$$

Differentiation of the free energy equation with respect to the component concentrations yields the chemical potentials. The calculated chemical potentials calculated are used to obtain the equilibrium concentrations of the coexisting anisotropic and isotropic phases described by

$$
\ln \left(v_{\mathrm{x}}{ }^{\prime} / v_{\mathrm{x}}\right)=(\mathrm{x}-1) v_{\mathrm{x}}-\left(\mathrm{y}_{\mathrm{avg}}-1\right) v_{\mathrm{x}}{ }^{\prime}+\ln \left(\mathrm{f}_{1}\right)
$$

where,

$$
f_{1}=\int_{0}^{y \max } \omega_{y} \exp [-a y] d y
$$

The distribution function, $\mathrm{f}_{1}$, must be recast in terms that can be determined experimentally. First, the disorder index ,y, and the angle, $\Psi$, are evaluated by considering projections of a rod on the transverse axes $\left(Y_{1}\right.$ and $\left.Y_{2}\right)$ to the preferred orientation axis. As before $\Psi$ is the angle between the rod domain axis and $\Phi$ is the angle of rotation about domain axis. The magnitude of the projection on each axis can be represented by

$$
\mathrm{y}=\left|\mathrm{y}_{1}\right|+\left|\mathrm{y}_{2}\right|=\mathrm{x} \sin \Psi(|\cos \Phi|+|\sin \Phi|)
$$

and averaging over $\Phi$ yields 


$$
\begin{gathered}
y=(4 / \pi) x \sin \Psi \\
\left(n_{x y} / n_{x}\right)=\left(f_{1}-1\right) \sin \Psi \exp [-(4 \pi \text { ax }) \sin \Psi]
\end{gathered}
$$

The integral involving the angular distribution $f_{1}$ can be evaluated with

$$
\left.f_{p}=\int_{0}^{\pi / 2} \sin ^{\mathrm{p}} \Psi \exp [-(4 \pi \mathrm{ax}) \sin \Psi] d \Psi\right)
$$

with $\mathrm{p}=1$. With $\mathrm{S}$ being the order parameter, the average values of $\sin \Psi$ and $\mathrm{y}$ are

$$
<\sin \Psi>=f_{3} / f_{1}=2 / 3(1-S) \quad y_{\text {avg }}=(4 / \pi)\left(f_{2} / f_{1}\right)
$$

Numerical solutions lead to the values of concentrations in the respective phases and of the disorder index $y_{\text {avg }}$ at equilibrium. This equation relates directly the variable concerning orientation in the lattice with the conventional order parameter. This order parameter is calculated without assumptions involving molecular interactions apart from excluded volume arising implicitly from the combinatorial procedure of filling the lattice.

The hard rod theories suggest that nematic to isotropic transitions can be predicted on purely steric grounds without attractive forces. However, experimental observations of liquid crystalline systems indicate predicted transition densities for hard rod models are too low and the order parameter calculated for the transition is too high [de Gennes, 1974b].

The models in hard rod theories are athermal due to the lack of attractive forces so that predicted transitions are independent of temperature. The next logical step is the incorporation of soft intermolecular forces to induce temperature dependence. To observe the effects of molecular attractions, perturbations are incorporated as discussed in the next section.

The extension of lattice theory to thermotropic systems is accomplished by incorporation of a Maier-Saupe type interaction of anisotropic dispersion forces between two rods averaged over all orientations to define a mean field. The term that is affected in the course of derivation is the orientational distribution $n_{x y} / n_{x}$. The volume of the system 
is the sum of the hard core volume of the rod molecules, $\mathrm{V}^{*}$, and a term representing the unoccupied sites in the lattice. The free volume is given in terms of the reduced volume, $\mathcal{V}=\mathrm{V} / \mathrm{N}^{*}$, in which the total number of lattice sites is $\mathrm{n}_{0}=\mathcal{V} \mathrm{xn}_{\mathrm{x}}$ [Ballauff, 1987].

The energy of interaction is taken to have both isotropic and anisotropic components,

$$
\varepsilon=\varepsilon_{\text {iso }}+\varepsilon_{\text {aniso }}(\Psi)
$$

where $\Psi$ is the angle with the domain axis of the cylindrical axis of a probe molecule (about which the interaction with a second molecule is averaged over all orientations).

The isotropic and anisotropic portions of the interaction energy are defined

$$
\begin{gathered}
\varepsilon_{\text {iso }}=\left(6 \alpha^{-2} \mathrm{C}\right) /\left(\nu_{\mathrm{r}} * 6\right) \\
\varepsilon_{\text {aniso }}(\Psi)=\left(\mathrm{kT}^{*} / \mathcal{N}\right) \mathrm{SP}^{2}(\cos \Psi)
\end{gathered}
$$

where $r^{*}$ is the intermolecular distance at $\mathrm{V}=1$ and $\mathrm{T}^{*}=\mathrm{a}$ characteristic temperature representing the strength of the anisotropic interaction

$$
\mathrm{kT}^{*}=2 \sigma \mathrm{p}^{*} \mathrm{~V}_{\mathrm{s}}^{*}(\Delta \alpha / \alpha)
$$

The term $\sigma$ is a geometrical factor depending on the distribution of rod segments in the system, $\Delta \alpha=\alpha_{11}-\alpha_{\perp}$ (the difference in the polarizabilities along and perpendicular to an individual segment in the molecule). $\mathrm{S}$ is the order parameter, and $\mathrm{P}^{2}(\cos \Psi)$ is the second Legendre polynomial.

Experiments on homologous series of rigid molecules show that the value of the nematic isotropic transition temperature, $\mathrm{T}_{n \mathrm{n}}$, can vary widely with the axial ratio of the molecule, while the interactions characterized by $\mathrm{T}^{*}$ remain relatively constant. These results confirm the importance of purely steric interactions upon the formation of the nematic liquid crystal phase. Statistical thermodynamic theory for thermotropic polymers containing flexible spacers indicate that the interconnectivity between mesogenic units increases the values of the order parameter and of the transition energies 
in comparison to molecular liquid crystals [Boehm et al., 1986]. Deuterium nuclear magnetic resonance experiments indicate that flexible spacers in polymeric liquid crystals exhibit more orientation relative to flexible chains attached to low molecular weight liquid crystals [Samulski, 1985].

The predictions by the theory depend upon the order parameter in the energetic description of the anisotropic interaction. The anisotropic part of the molecular interaction disappears as the order parameter approaches zero.

\subsubsection{Elastic Continuum Theory of Nematics}

The average local direction of the anisotropic molecules is defined as the director. Fluctuations of the director do not appear to influence the first order phase transition from a nematic to the isotropic liquid. Therefore, the standard approach is to describe the phase transitions in terms of a mean molecular field or phenomenological theories and use a continuum description for distortional fluctuations of the director field [Warner, 1984]. The same continuum theory can be applied to both monomolecular and polymer liquid crystals without concern for theoretical differences concerning the origin of their phase behavior. The main assumptions are that the order parameter $\mathrm{S}(\mathrm{T})$ does not depend upon position in the nematic phase and that the average orientation described by the tensor field, $\mathbf{N}(\mathbf{r})$, varies slowly in space.

\subsubsection{Frank Free Energy Density}

The continuum theory introduced by Frank to account for the equilibrium form of curvatures of the director field is analogous to elasticity theory [Frank, 1958, Vertogen, 1985; and Chandrasekhar, 1977]. In the classical elasticity theory of solids, restoring forces are assumed to resist changes in the distances between material points after homogeneous strains are applied. In distorted liquid crystals there are no permanent forces opposing the distance between points, but there are restoring twisting 
or bending moments that directly oppose the curvature. Frank [1958] assumed a linear relationship between the twist and bending moments and curvature strains. If the curvature strains are sufficiently small in magnitude the defined relationship is a statement equivalent to Hooke's Law.

The free energy density is written in conventional vector notation first given by Oseen

$$
f_{d}=1 / 2 K_{1}(\operatorname{div} \mathbf{n})^{2}+1 / 2 K_{2}(\mathbf{n} \cdot \operatorname{curl} \mathbf{n})^{2}+K_{3}(\mathbf{n} \times \operatorname{curl} \mathbf{n})^{2}
$$

where $K_{1}=2\left(k_{1}+k_{2}\right), K_{2}=2 k_{2}$, and $K_{3}=2\left(k_{2}+k_{3}\right)$. The constants $K_{1}, K_{2}$, and $K_{3}$ are the elastic constants for splay, twist, and bend, respectively. Figure 1.2 shows the deformations associated with three constants [Vertogen and de Jeu, 1985].

The relative magnitudes of the Frank elastic constants can be calculated for model nematics assuming a given director field [Straley, 1973, Priest, 1973, Nehring and Saupe, 1971, and Nehring and Saupe, 1972]. The equal constant assumption $\left(\mathrm{K}_{1}=\mathrm{K}_{2}=\mathrm{K}_{3}\right)$ simplifies calculations of equilibrium structures around singularities or disclinations and other distortions occurring in director fields. The Frank elastic constants $(\mathrm{k})$ have values of typically $10^{-6}$ dynes $/ \mathrm{cm}^{2}$ and therefore store minuscule energies compared to ordinary elastic constants $(E)$ of macroscopic solid structures with values on the order of $10^{10}$ dyne/ $\mathrm{cm}^{2}$. Frank [1958] calculated that the ratio of the free energy due to deformation due to Frank elasticity, $g_{k}$, to ordinary elasticity, $g_{E}$ arising from bending of a beam with thickness a to a radius of $\mathrm{R}$ :

$$
\mathrm{g}_{\mathrm{k}} / \mathrm{g}_{\mathrm{E}}=\frac{12 \mathrm{k}}{\mathrm{Ea}^{2}}
$$

On submicron length scales the values of these restoring energy constants of curvature deformations of the director field do become significant [Frank, 1958]. Only a very small fraction of the total energy is held at molecular dimensions in which would involve deviations in the order parameter [de Gennes, 1974a]. 

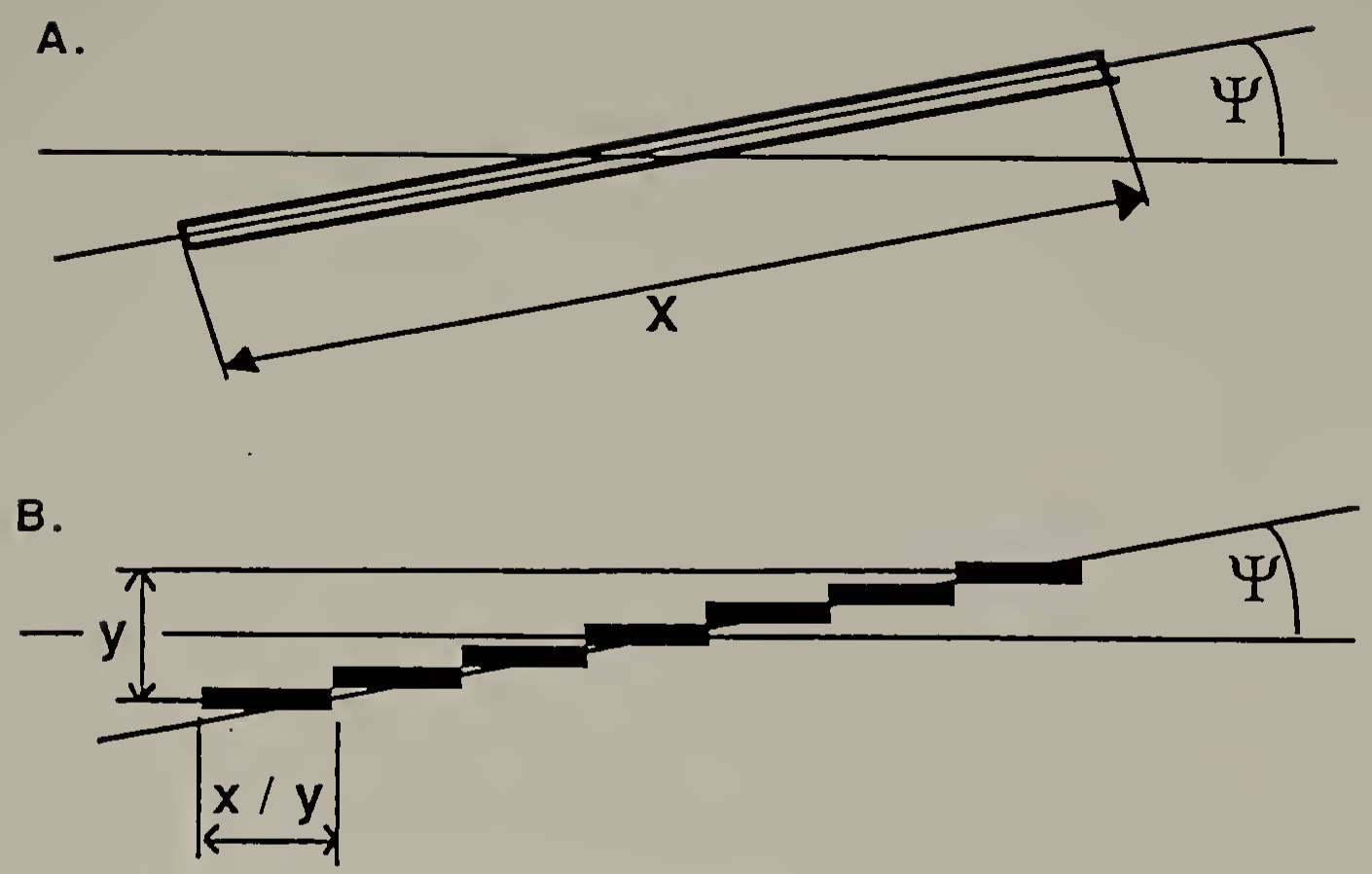

Figure 1.1 (A) A rodlike particle oriented at angle $\psi$ to the preferred axis of the domain. (B) The representation of the particle as y sequences or submolecule, each parallel to the domain axis. Explicitly, $\psi$ is the angle of inclination to the $\mathrm{x}$ axis and $\mathrm{x}$ is the length of the molecule.

A

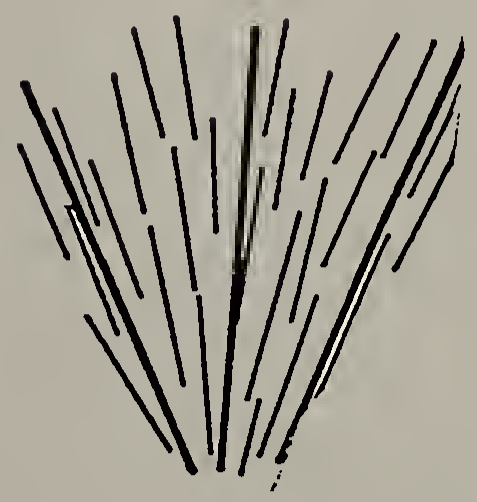

B

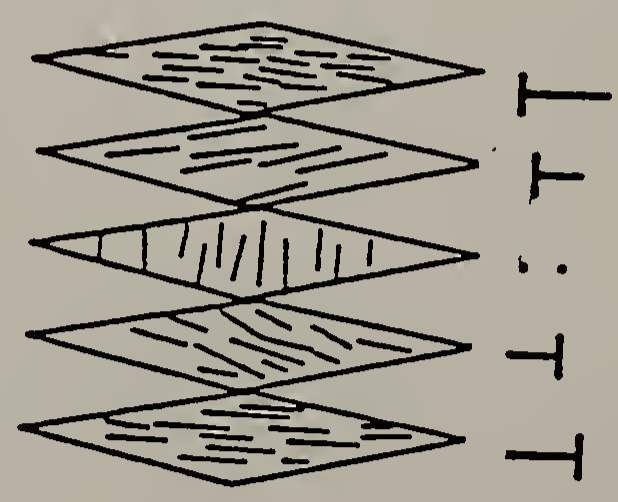

C

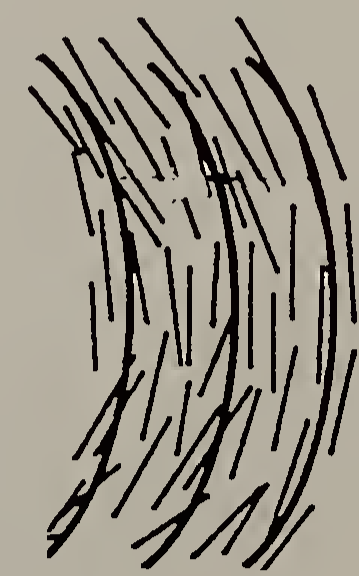

Figure 1.2 The three basic deformations of a uniaxial nematic (A) splay, (B) twist, and $(C)$ bend. 


\subsubsection{Surface terms in Frank continuum theory}

Nehring and Saupe [1971] argue that second order terms need to be incorporated into the free energy expression. They derived both from molecular and phenomenological principles the following equation for the free energy density for an axial nematic.

$$
f_{d}=K_{1}(\operatorname{divn})^{2}+K_{2}(n \cdot \operatorname{curln})^{2}+K_{3}(n \times c u r l n)^{2}+2 K_{13}(n \cdot g r a d \operatorname{div} n)^{2}
$$

with

$$
\mathrm{K}_{1}=\mathrm{K}_{11}-2 \mathrm{~K}_{13} \quad \mathrm{~K}_{3}=\mathrm{K}_{33}-2 \mathrm{~K}_{13}
$$

In order to calculate director fields for a specific set of elastic constants the free energy density function must be minimized. The argument against including the such terms as $K_{13}(\mathbf{n} \cdot \operatorname{grad} \operatorname{div} \mathbf{n})^{2}$ is that they can be transformed into surface integrals by Gauss's theorem and therefore do not contribute to the Euler-Lagrange equation of the bulk [Nehring and Saupe, 1971]. However, it is suggested by Nehring and Saupe that the free energy should contain such terms on the basis that they may affect the equilibrium structure because they still contribute energy.

There is much belligerent rhetoric in the literature concerning the solutions for free energy densities containing surface terms with the $K_{13}$ constant [Hinov, 1989, Baldero and Oldano, 1989]. Other methods of addressing the problem involve separation of the bulk free energies and surface energies by phenomenological, structuremodel, or thermodynamic means. The direction imposed upon the bulk director by conditions at the interface are framed as boundary conditions from which equilibrium structures can be calculated. The determination of equilibrium structures of the bulk will now be addressed with subsequent discussion of the influence of the surface of the nematic on the properties of the material.

The energy dependence upon director distortions is considered to be orders of magnitude smaller than the energy related to the order parameter. The energy terms can be considered to be independent and the notion of decoupling is valid along with the use 
of a separate continuum theory (reviewed in the previous section) to describe the director. However, without fields or boundaries, the equilibrium director field is undetermined even with the $\mathbf{n}^{2}=1$ constraint [Goosens, 1985].

We let $\phi$ be the angle of the director with a fixed direction in the xy plane and assume the elastic constants to be equal. In thin films the director field is two dimensional with one degree of freedom, $\phi$, the angle of the director with a reference axis. The director takes the form.

$$
\mathbf{n}=\cos \phi \mathbf{i}+\sin \phi \mathbf{j}
$$

The Euler-Lagrange differential equation that minimizes the total free energy is the Laplacian of $\phi$

$$
\left(\nabla^{2} \phi\right)=0
$$

The equal elastic constant assumption will only change some of the details of the solution, and the essential geometry will be preserved. Sometimes the stability of the solution requires inequality of the constants [Meyer, 1973]. Without boundary conditions the direction of minimum free energy for the molecular field is undetermined. In a thin film there is the implicit assumption that the director field is confined within the plane but that the orientation is undefined. The structure around a discontinuity or singularity in the director field is given by the a set of solutions

$$
\phi=s \theta+\phi_{0}
$$

The value $s$ is defined as the strength of the disclination given by the rotational sense of the structure normalized by $2 \pi$. The director fields described by these solutions are called disclinations which are observed in great numbers if the orientation direction with respect to the surfaces in thin films is degenerate. The elastic energy of a disclination is calculated to be infinity as the singularity is approached. A core radius is defined to adjust with an energy per unit length similar to description given screw dislocations in a solid [Kle man, 1983]. 
The actual structures of disclinations are dependent upon existence of surface interactions of the nematic. Strong interactions make singularity points less likely and cause the formation of surface defect lines [Kle man, 1983]. The energy and structure of these defects depend upon the anchoring energy and the presence of an easy axis. Even in the absence of defects, the equilibrium director field in the bulk is heavily influenced by the boundary conditions that depend upon the orientation at which the liquid crystal is anchored. These terms are intimately related to the surface free energy of nematics liquid crystals and will be discussed in the next and subsequent sections.

\subsubsection{The Nematic Interface}

The existence of singular points at the surface of thin films depends on a lack of preferred direction or a strong degeneracy of anchoring [Kléman, 1983]. The term anchoring refers to how orientation of the director is affected when in contact with an exterior surface. Planar anchoring is orientation of the director parallel to the plane of the interface. Homeotropic anchoring is perpendicular alignment of the director relative to the surface.

The director orientation at the surface can be defined by two angles, using spherical coordinates, an angle with the surface normal, $\phi(0<\phi<\pi)$ and an azimuthal angle in the plane of the surface, $\theta$. With this coordinate system, homeotropic anchoring occurs with $\phi=0$; and planar anchor is when $\phi=\pi / 2$. A definite alignment $\theta_{0}, \phi_{0}$ imposed upon the nematic at the surface is called the easy-axis [Kle man, 1983]. If the angle $\theta$ is undefined for a specified easy-direction $\phi_{0}$, there exists a cone of degeneracy.

An easy-axis usually occurs when a surface has anisotropic character that it can impose upon director. The nematic orients spontaneously in the direction imposed locally by the substrate to minimize surface energy. Examples that are cited by de Gennes [1974a] are: 
1) When deposited on a crystal face some nematogens may associate closely with certain crystallographic directions. The existence of more than one easy axis is termed to be degenerate.

2) A surface that is carefully rubbed in one direction. The mechanism for this phenomenon is uncertain, but speculations include the deposition of a fatty acid microlayer or the formation of a directional surface topography.

3) By the evaporation of thin films on a glass surface at an oblique angle, one can set the easy-axis to be parallel or at a tilt with respect to the plane of the glass depending upon the angle of incidence of the deposition.

4) Surfactants can be used to determine how the nematic molecules orient at the surface. This is one method of producing homeotropic anchoring of the liquid crystal. Weak homeotropic anchoring is observed for some very clean surfaces.

5) With dispersion of nematic liquid crystals into isotropic medium one can observe the effect of geometry upon the director field and the limits of its distortions exemplified by the surface orientation and the types of defects that arise in the nematic droplets.

Surface anchoring can be loosely termed strong or weak depending on how well the director correlates with the easy axis. Highly degenerate anchorings are classified as weak as is the case in tilted nematics or planar nematics without a specified directions of orientation imposed by the surface [Yokoyama, 1988]. Uncertainty persists as to whether the dominant mechanism of the observed alignment is due to topological features or molecular scale interactions of oriented species present at the surface.

The combination of homeotropic and planar anchoring on opposing interfaces can be used as boundary conditions to calculate equilibrium director fields in two dimensions [Meyer, 1973]. The solutions indicate that the exact configuration of the director field depends upon the ratio of splay to bend. 
Theoretically, the same method can be used to determine the twist elastic constant using a thin nematic film between two surfaces with planar anchoring, but are twisted about the normal of the two parallel surfaces. If one measures the torque caused by the imposed distortion of the director the value of the constant is determined. In actuality, the needed torque is determined by using magnetic fields [Chandrasekhar, 1977]. With these various boundary conditions and the stipulation of strong anchoring, it is possible to obtain values of Frank elastic constants. For weak anchoring such experiments specify values of an anchoring energy as a threshold at which the distortions in the bulk due to the surface anchoring are nulled by the magnetic field [de Gennes, 1974b].

The anchoring energy is defined in terms of the excess surface energy. One term that might contribute to an excess orientation energy is the order parameter. However, the amount of orientational excess energy is difficult to quantify experimentally. Theoretical calculations indicate that density fluctuations dominate the surface tension of a nematic with an isotropic phase [Goosens, 1985 and Telo da Gama, 1984a]. Anchoring energy is usually only associated with the director distortions caused by the perturbation of the easy axis into the bulk.

The anchoring energy, $E_{\mathrm{a}}$, is implicitly assumed by this relation to be isotropic and does not depend upon the direction or the mode deformation. However, one would expect that the various deformations should lead to different values of anchoring energy. The strength of anchoring is a classification concerning the relative amount of interaction of the nematic material with the surface. One usually finds strong anchoring of liquid crystals at crystalline walls and weak anchoring for polished surfaces such as glass [de Gennes, 1974b].

For nematic droplets the area of minimum surface energy will dictate the a spherical shape, but the elastic constants of the material determine the defect structure occurring in distortions of the director field [Drzaic, 1988; Kle man, 1983; Meyer, 1982]. 
There are citations with a languid analogy to Wulff construction in solid crystals alluding to nonspherical equilibrium shapes in some lyotropic liquid crystals [Chandrasekhar, 1977].

The order parameter has been theoretically evaluated to increase in the vicinity of a surface to reduce the free energy [Goosens, 1985]. Expressions of the surface energy are obtained as expansions of the order parameter in which the coefficients depend upon the orientation of the director at the interface. These calculations yield surface energytemperature relations with discontinuities found at the nematic-isotropic transition. Comparisons of surface energy calculations for different director configurations, (parallel or perpendicular to the liquid crystal-free vapor interface, for instance), give suggested directions of the easy-axis at the surface [Croxton, 1980].

Theories based on Van der Waals models give similar relations for surface energy-temperature relations, but fall short in predictions of the easy-axis direction [Telo de Gama, 1984b; Croxton, 1980]. These studies deal mainly with molecular order at the nematic-isotropic transition. Though findings have implications on the presence of order at the interface of an isotropic phase and the direction of the easy axis, there is some question whether measurements of excess order and energy at the interface will yield information concerning the anchoring energy [Goosens, 1985].

Models concerning the nematic interfaces in the literature, range from the molecular perspective found in mean field and statistical mechanical theories [Croxton, 1980; Telo de Gama, 1984b] to phenomenological theories involving characteristic expansion variables such as the order parameter [Goosens, 1985]. In these systems the form of the function for the surface energy can be considered to be in a sense arbitrary with the restriction that the model realistically depicts some aspect of the interface [Yokoyama, 1988]. 


\subsection{Blend Mixing}

\subsubsection{Phase diagrams}

The morphology of the spin dope or melt is influenced by the nature of the equilibrium phase diagram determining the number of domains, the surface energy of the interfacial area, and both the amount and method of mechanical mixing [La Mantia et al., 1990; Bassett and Yee, 1990]. The major difference in miscibility behavior in mixtures in small molecules and polymers is the numbers of molecules per unit volume. Phase behavior is governed by variations in polymer molecular weight and distribution [Koningsveld, 1989, Flory, 1956]. Lattice theories such as the one proposed by Flory are used to define expressions for $\Delta \mathrm{G}$, the Gibbs free energy of mixing such as:

$$
\Delta \mathrm{G} / \mathrm{NRT}=\left(\Phi_{1} / \mathrm{m}_{1}\right) \ln \Phi_{1}+\left(\Phi_{2} / \mathrm{m}_{2}\right) \ln \Phi_{2}+\mathrm{g} \Phi_{1} \Phi_{2}
$$

Where $\mathrm{T}$ is temperature,

$\mathrm{R}$ is the gas constant,

$\Phi_{1}=\mathrm{n}_{1} \mathrm{~m}_{1} / \mathrm{N}=\mathrm{x}_{1} /\left(\mathrm{x}_{1}+\mathrm{x}_{2} \mathrm{~m}_{2} / \mathrm{m}_{1}\right)$,

$\mathrm{N}$ is the total amount of material in moles of lattice sites,

$\mathrm{n}_{1}$ is the amount of component 1 in moles,

$\mathrm{m}_{1}$ is number of lattice sites occupied by a molecule, and

$\mathrm{g}$ is an interaction parameter in which for a first approximation

$\mathrm{g}=\mathrm{h} / \mathrm{T} ; \mathrm{h}=\mathrm{z} \Delta \mathrm{w}_{12} / \mathrm{RT}$,

$\mathrm{z}$ is the lattice coordination number and

$\Delta w_{12}$ is the internal energy change for breaking one mole of 1-1 and 2-2 nearest neighbor contacts and creating one mole of 1-2 contacts.

The enthalpy of mixing, $\Delta \mathrm{H}$ can be related to $h$ by the relation:

$\Delta \mathrm{H} / \mathrm{NR}=\mathrm{h} \Phi_{1} \Phi_{2}$ 
The enthalpy of mixing for macromolecules does not differ greatly from the values seen for small molecules. The difference in miscibility between these two systems is mainly due to the reduced combinatorial entropy of mixing per site caused by the connectivity in the former leading to an unstable entropy-energy balance in polymer systems [Koningsveld, 1989]. One phase polymer blends are characterized by the presence of specific interactions between the chemical species that lead to negative $h$ values.

Such treatments have been modified to address the mixing coil polymer with LCPs [Matheson and Flory, 1981]. The combinatorial terms are adjusted to account for placing the rods on the lattice as previously reviewed. The theory suggests that the addition of side chains to rigid molecules increases the predicted miscibility with coil polymers [Ballauff, 1987].

\subsubsection{Mixing}

Aspects of polymer mixing to give indication of what types of interfaces and domain shapes might arise from the rheological processing of LCP blends with isotropic materials. Also pertinent is the recent experimental observations that link the bending distortions to the fundamental nature of nematic elasticity and viscosity.

The equilibrium phase diagram will determine if miscibility of the two materials is attainable, although current experimental work suggests that observed polymer miscibility can be influenced by dynamic conditions such as shear [Katsaros, et al., 1989]. If a polymer blend is immiscible the shape of the observed domains of the respective polymers depends upon the type, magnitude, and amount of deformation and subsequent thermal history determining the allowed relaxation.

The formation of structure in immiscible polymer blends under with various flow histories has been studied experimentally with SEM and TEM examinations [Frederico, 
1989; La Mantia, 1990; Bassett and Yee; 1990]. There is almost no experimental data studying the droplet or microstructure deformation with clearly defined histories of deformation. Theoretical simplifications are a necessity, so analysis and experimentation center on simple shear and plane hyperbolic flows [Ottino, 1989].

For immiscible fluids that have similar properties and no interfacial energy, there is no resistance to deformation and the interface and the material elements are called passive. The passive material element (length $=d \mathbf{x}$ and orientation $\mathbf{m}=\mathrm{dx} / \mathrm{d} \mathbf{x} \mid$ ) deforms according to the equations:

$\mathrm{D}(\ln \lambda) / \mathrm{Dt}=(\nabla \mathbf{v}): \mathrm{mm} \quad$ (rate of change of length stretch)

$\lambda=\lim |\mathrm{d} \mathbf{x}| /|\mathbf{d} \mathbf{X}|$

$\nabla \mathbf{v}=$ the velocity gradient

$\mathrm{Dm} / \mathrm{Dt}=(\nabla \mathbf{v})^{\mathrm{T}} \cdot \mathbf{m}-(\mathbf{D}: \mathbf{m m}) \mathbf{m} \quad$ (rate of change of orientation)

$\mathrm{D}=1 / 2\left(\nabla \mathbf{v}+(\nabla \mathbf{v})^{\mathrm{T}}\right) \quad$ (the stretching tensor)

If the material has an internal resistance to the deformation such as viscosity or interfacial energy, the material element is said to be an active microstructure. Such active elements lead to more complicated vector equations than the ones given above [Ottino, 1989].

Most analysis investigate the role of the tensor characterizing the imposed flow, $\mathbf{L}(=\mathbf{L}(\mathbf{X}, \mathrm{t})=\mathbf{D}+\Omega)$, in stretching and break up of a drop. $\Omega$ is the vorticity or the spin tensor and $\mathbf{D}$ is as previously defined. The main parameters for a system with a characteristic shear rate $S,\left((D: D)^{1 / 2}\right)$, a, length scale of the microstructures, $\mu_{i}$ and $\mu_{e}$, the respective viscosities of the drop and the external medium, and $\sigma$ the interfacial energy. The main parameters for such a system are defined as:

1) the capillary number, $\mathrm{Ca}=\mathrm{S} \mu_{\mathrm{e}} / \sigma$, or the ratio of viscous to surface tension forces,

2) the viscosity ratio, $p=\mu_{i} / \mu_{e}$,

3) the imposed flow $L(=L(X, t)$ 
4) the initial drop shape.

The first main finding is that for low capillary numbers, $(\mathrm{Ca}<1)$, in both simple shear and planar elongational flows, deformation increases linearly with $\mathrm{Ca}$. Long slender droplets result from low values of the viscosity ratio, $\mathrm{p}$ and large values of $\mathrm{Ca}$. Secondly, a critical capillary number, $\mathrm{Ca}_{c}$, exist such that the surface tension forces are surpassed by the viscous forces and the drop will continually elongate without break up in steady flow. Before the $\mathrm{Ca}_{c}$, the extent of deformation is a function of the strain rate. Finally, for simple shear dispersed droplets will not break up if the value of the viscosity ratio is value is greater than approximately 4 .

\subsubsection{Polymer Melts}

Experiments characterizing the initial condition of the polymer melt mixture or the geometry of the dispersed component is essential to understanding the deformation of the dispersed components in a defined flow field. Besides the general drop shape methods applied to characterize the interfacial free energy, the value of $\mathrm{Ca}$ can be estimated.

In fiber spinning with low interfacial energy between components high draw ratios would create fibrils of the dispersed phase if the viscosity of dispersed component is comparable to the external component. Both materials should have similar viscosities for fibril formation. Large interfacial energies between the domains will result in the deformed, elongated domains to break into droplets. Fibrils are usually observed in such cases where elongational flow dominates if interfacial energy is not too great [Bassett and Yee, 1990].

More complicated deformation histories are observed in the injection molding process of polymer blends and involve more than the understanding of deformation of material elements in an isothermal flow field. The domain morphology resulting from 
injection molding is complicated and a variety of structures are usually observed across the part. In the injection molding of pure liquid crystals, the layered morphologies range from a highly oriented skin to an observed lack of orientation at the core of the processed specimens [Hedmark et al., 1988].

Characterization of shear and elongational flows of liquid crystals indicate that elongational flow is more effective in orienting LCPs than shear. For elongational flow the draw ratio is the characteristic parameter which has a direct correlation with orientation,

$$
\tan P_{x}=\left(\tan P_{x=0}\right)\left(D_{R}-\lambda\right)
$$

where $\mathrm{P}_{\mathrm{X}}$ is the orientation angle that an orienting particle makes with the elongation direction $\mathrm{x}$.

$P_{x=0}$ represents any intrinsic orientation before elongational flow.

$D_{R}$ is the draw ratio.

$\lambda$ is a characteristic parameter that relates to the orientability of the material.

The total shear determines the amount of orientation, so only high shear rate processes such as injection molding are capable of imparting the orientation comparable to highly drawn fibers [Kenig 1987, Kenig 1989].

The banded structure observed in thermotropic and lyotropic polymers subjected to flow is thought to be consequence of relaxations or flow instabilities which may be related to the Frank elastic constants [Thomas and Wood, 1985, Meyer, 1973]. The elastic constants of splay and bend and viscosity coefficients of liquid crystals corresponding to comparable flow deformations have been shown to correlate with molecular structure in a lyotropic liquid crystal PBLG. The bend coefficient is dependent upon the molecular weight up to the persistence length of the polymer. However, the splay coefficient is shown to have a molecular weight dependence well past the persistence length [Lee and Meyer, 1973]. 
Ballauff, M., Journal of Polymer Science, Polymer Physics Edition, 25, pp. 739 747(1987). "Compatibility of Coils and Rods Bearing Flexible Side Chains".

Ballauff, M., Molecular Crystals Liquid Crystals, 168 , pp. 209-228(1989). "The Floury Lattice Model of Nematic Fluids".

Barbero, G. and Oldano, C., Molecular Crystals Liquid Crystals, 168 , pp. 1-5(1989). "On the $\mathrm{K}_{13}$-Dependent Energy Term in Nematic Liquid Crystals".

Bassett, B. R. and Yee, A. F., Polymer Composites, 11, pp. 10-18(1990). "A Method of Forming Composite Structures Using In Situ- Formed Liquid Crystal Polymer Fibers in a Thermoplastic Matrix".

Boehm, R. E., Martire, D. E., and Madhusudana, N. V., Macromolecules, 19, pp. 2329-2341 (1986). "A Statistical Thermodynamic Theory of Thermotropic Linear Main-Chain Polymeric Liquid Crystals".

Chandrasekhar, S., Liquid Crystals, Cambridge University Press, Cambrigde, 1977.

Clark, M. G., Molecular Crystals Liquid Crystals, 127, pp. 1-41(1985). "Macroscopic Properties of Liquid Crystals".

Cotter, M. A., Phil. Trans. R. Soc. Lond, A309, pp. 127-144 (1983) "The Van der Waals Theory of Nematic Liquids".

Croxton, C., Molecular Crystals Liquid Crystals, 59, pp.219-240(1980). "Statistical Thermodynamics of the Liquid Crystal Surface, Smectic, Nematic and Isotropic System".

Doi, M. and Edwards, F. S., Theory of Polymer Dynamics, Claredon Press, Oxford, 1986.

Drzaic, P., Molecular Crystals Liquid Crystals, 154, pp. 289-306(1988). "A New Director Alignment for Droplets of Nematic Liquid Crystal with Low Bend to Splay Ratio".

Flory, P. J. and Ranch, G., Molecular Crystals Liquid Crystals 54, 289-310(1979). "Theory of Systems of Rodlike Particles, I. Athermal systems".

Flory, P. J. and Ronca, G., Molecular Crystals Liquid Crystals 54, pp. 311-330(1979). "Theory of Systems of Rodlike Particles".

Flory, P. J., Proceedings of the Royal Society, A, 234, pp. 73-89(1956). "Phase Equilibria in Solutions of Rod-like Particles".

Frank , F. C., Discussions of the Faraday Society, 25, pp. 19-28(1958). "On the Theory of Liquid Crystals". 
Frederico, O., Ph. D. Dissertation, Polymer Science and Engineering Department, University of Massachusetts, Amherst, MA (1989). "In-situ Composites of a Thermotropic Liquid Crystalline Polymer and Polycarbonate: Processing, Morphology and Properties".

Gennes, P. G. de, Liquid Crystals and Plastic Crystals, Volume 1 , Ellis Horwood Limited, Chichester, (1974), G. W. Gray and P. A. Winsor, editors. "Principles of Continuum Theory".

Gennes, P. G. de, The Physics of Liquid Crystals, Clarendon Press, Oxford(1974).

Goosens, W. J. A., Molecular Crystals Liquid Crystals, 124, pp. 305-331(1985). "Bulk, Interfacial and Anchoring Energies of Liquid ".

Hinov, H. P., Molecular Crystals Liquid Crystals, 168, pp. 7-12(1989). "Comment on the Criticism of the One-Dimensional Solution of the $\mathrm{K}_{13}$ Elastic Problems in Nematics".

Hedmark, P. G., Lopez, J. M. R., Westdahl, M., Werner, P., Jansson, J., and Gedde, U. W., Polymer Engineering and Science, 28(19), pp. 1248-1259(1988). "Microstructure in Injection Molded Samples of Liquid Crystalline Poly(PHydroxy-Benzoic Acid-Co-Ethylene Terephthalate)".

Katsaros, J. D., Malone, M. F., and Winter, H. H., Polymer Engineering and Science, 29 , pp. 1434-1445(1989. "The Effects of Flow on Miscibility in a Blend of Polystyrene and Poly(Vinyl Methyl Ether)".

Kenig, S., Polymer Engineering and Science 27,pp. 887-892(1987). "Orientablility of Liquid Crystal Polymers in Elongational Flow".

Kenig, S., Polymer Engineering and Science, 29, pp. 1136-1141(1989). "ShearInduced Orientation in Liquid Crystalline Polymers".

Kle'man, M., Points, Lines and Walls, John Wiley and Sons, New York, 1983.

Koningsveld, R., Pure and Applied Chemistry, 61, pp. 1051-1064(1989). "Thermodynamics of Macromolecular Systems".

La Mantia, F. P.; Valenza, A.; Paci, M.; and Magagnini, P. L., Polymer Engineering and Science, 30, pp. 7-12(1990). "Rheology-Morphology Relationships in Nylon 6/Liquid-Crystalline Polymer Blends".

Lee, Sin-Doo and Meyer, Robert B., Physical Review Letters, 61, pp. 2217-2220. "Crossover Behavior of the Elastic Coefficients and Viscosities of a Polymer Nematic Liquid Crystal".

Luckhurst, G. R.; Nuclear Magnetic Resonance of Liquid Crystals, edited by J. W. Emley, pp. 53-83, NATO ASI Series, Series C: Mathematical and Physical Sciences Volume 141., 1983, D. Reidel Publishing Company, Dordrecht, Holland. "The Molecular Field Theories of Nematics: Systems Composed of Uniaxial, Biaxial or Flexible Molecules". 
Luckhurst, G. R., Nuclear Magnetic Resonance of Liquid Crystals, edited by J. W. Emley, pp. 85-97, NATO ASI Series, Series C: Mathematical and Physical Sciences Volume 141., 1983, D. Reidel Publishing Company, Dordrecht, Holland. "The Landau-de Gennes Theory of Liquid Crystals".

Matheson, R. R. and Flory, P. J., Macromolecules, 14, pp. 954-960(1981). "Statistical thermodynamics of Mixtures of Semirigid Macromolecules: Chains with Rodlike Sequences at Fixed Locations".

Meyer, Robert B., Molecular Fluids, Gordon and Breach Science Publishers, London, R. Balian and G. Weill,(1973) editors, pp. 271-343. "Structural Problems in Liquid Crystal Physics".

Meyer, Robert B., Polymer Liquid Crystals, Academic Press, New York, 1982, A. Ciferri, W. R. Krigbaum, and R. B. Meyer, editors. Chapter 6, "Macroscopic Phenomenon in Nematic Polymers".

Nehring, J., Saupe, A., Journal of Chemical Physics, 54, pp. 337-343(1971). "On the Elastic Theory of Uniaxial Liquid Crystals".

Nehring, J., Saupe, A., Journal of Chemical Physics, $\underline{56}$, pp. 5527-5528(1972). "Calculation of the Elastic Constants of Nematic Liquid Crystals".

Ottino, J., The Kinematics of Mixing: Stretching Chaos, and Transport, Cambridge University Press, Cambridge 1989.

Priest, R. G., Physical Review A, 7, pp. 720-729(1973). "Theory of the Frank Elastic Constants of Nematic Liquid Crystals".

Samulski, E. T., Polymeric Liquid Crystals, Plenum Press, New York and London 1985, Alexandre Blumstein, editor. "Some Physico-Chemical Aspects of Polymeric Liquid Crystals".

Straley, J. P., Physical Review A, 8, pp. 2181-2183(1973). "Frank Elastic of the HardRod Liquid Crystals".

Telo da Gama, M. M., Molecular Physics, 52, pp. -611-630 (1984). "The Interfacial Properties of a Model of a Nematic Liquid Crystal II. Induced Orientational Order and Wetting Transitions at a Solid-Fluid Interface ".

Telo de Gama, M. M., Molecular Physics, 52, pp. 585-610 (1984). "The Interfacial Properties of a Model of a Nematic Liquid Crystal I. The Nematic-Isotropic and the Nematic-Vapor Interfaces".

Thomas, E. L. and Wood, B. A., Faraday Discussions of the Chemical Society, 79, pp. 229-239 (1985). " Mesophase Texture and Defects in Thermotropic LiquidCrystalline Polymers".

Yokoyama, H., Molecular Crystals Liquid Crystals, 165, pp. 265-316(1988). "Surface Anchoring of Nematic Liquid Crystals". 
Vertogen, G., de Jeu, W. H., Thermotropic Liquid Crystals, Fundamentals, Springer-
Verlag, Berlin, 1985.

Warner, M.; Molecular Physics, 52, 677-690(1984). "Fluctuations, Mean Fields and the Order Parameters in Nematics".

Wood, B. A., Ph. D. Dissertation, Polymer Science and Engineering Department, University of Massachusetts, Amherst, MA (1985). "Ultrastructural Studies of Thermotropic Liquid Crystalline Polymers". 


\section{CHAPTER 2}

\section{INFILTRATION OF PBZT FILMS WITH SOL-GEL GLASS}

\subsection{Introduction}

Poly(p-phenylene benzobisthiazole) or PBZT is a lyotropic rigid rod polymer that when spun into fibers shows outstanding unidirectional strength and modulus and high heat resistance [Allen, 1983]. Lusignea et al. [1989] have made quasi-isotropic PBZT films possessing high strength and modulus with controllable planar orientation [Lusignea, 1989, Kovar et al., 1989]. Such films are produced by extruding PBZT solution through a counter rotating annular die assembly followed by coagulation. The balanced mechanical properties, the high temperature stability, along with the dielectric and optical properties of these biaxially oriented films will make them candidates for fabricating composite materials and structures with unique characteristics. These films are being investigated as reinforcement for a variety of matrices [Newman et al., 1991]. The compressive strength of rigid rod polymers is generally about one tenth of the tensile strength and has been shown to result from elastic instabilities associated with low shear modulus [DeTeresa, S. J., Allen, 1987, Allen, 1989). A proposed way of improvement of compressive strength of PBZT is to epoxy impregnate the water swollen microfibrillar structure observed in rigid rod polymers before collapse upon drying [Cohen, Farris and De Teresa, 1986, 1989, 1990, and 1992, Cohen, 1987]. Pottick [1986] showed that PBZT fibers show very high strength while they were still swollen with water after coagulation and prior to drying or heat treatment. This is direct mechanical evidence for the existence of the microfibrillar network. Such an approach could improve the compressive strength by increasing fibril interactions and correspondingly the shear modulus. 
Lusignea et al. fabricated biaxial oriented films by using a counter rotating die and characterized the mechanical properties of these films and their composites [Lusignea, 1989, Kovar et al., 1989, Newman et al., 1991]. This chapter presents the morphology of biaxially oriented films and PBZT/glass composite films fabricated by permeating the as-coagulated water swollen PBZT biaxially oriented films with sol/gel glass systems. Alcohol soluble sol-gel precursors will infiltrate into the swollen film which after drying and a heat treatment will yield a PBZT/glass composite that hopefully exhibits good compressive properties.

\section{$2.2 \quad$ Experimental}

\subsubsection{Materials}

After extrusion through an annulus composed of counter rotating cylinders, subsequent coagulation, and stage drying cure, the biaxial films have a specific curl (See Figure 2.1). Here the inner surface refers to the concave side of the film and the outer surface refers to the convex side as shown in Figure 2.1B. Figure 2.1C illustrates the coordinate system used to describe orientation of the films for X-ray and microscopy experiments.

Table 2.1 lists the samples characterized in this study. Stage drying is the process that removes the water from the film while on a frame under tension using a specific applied heating program [Lusignea, 1986]. The composites contain sol glasses of different densification temperatures chosen regarding the degradation temperature of PBZT [Kovar, 1986, Kovar and Lusignea, 1986].

Lusignea showed that the biaxial PBZT films have a layered structure [Lusignea, 1989]. The SEM image in Figure 2.2A shows cut biaxial PBZT consists of fibrils with layers showing two distinct preferred orientations. The fracture surface in Figure 2.2B suggests there is a region within the film where the fibril orientation twists between the 
two preferred directions. The micrograph shows that within this zone there is a gradual change in orientation through the film thickness resulting from the flow field between the counter rotating cylinders during fabrication.

The as-coagulated PBZT films are swollen with water and can be stored in this state. The films undergo irreversible collapse upon the removal of water by drying or by osmotic pressure if placed in a liquid with a significant difference in polarity. To obtain sol-gel glass infiltrated PBZT films, water must be replaced using a series of exchanges of alcohol/water mixtures followed by a similar sequence with sol-gel precursor/alcohol mixtures.

The method outlined by Cohen describes the infiltration of PBZT fibers and films with epoxy resins [Cohen, 1989, Cohen, Farris and De Teresa, 1986, 1989, 1990, and 1992, Cohen, 1987]. Using a progression of ethanol/water mixtures of increasing ethanol content, ethanol replaces the water in the as-coagulated swollen film. Continuing the procedure with mixtures of Epon 828 epoxy, hexamethylene diamine curing agent and ethanol yields a PBZT/epoxy composite film [Cohen, 1987].

Employing mixtures of alcohol soluble sol-gel glass precursors in these procedures produces PBZT/glass composite films upon drying and curing. Figure 2.3 summarizes the operation of infiltrating the microfibrillar network of rigid rod polymers.

\subsubsection{Wide Angle X-ray Scattering (WAXS)}

WAXS diffraction patterns are from a Statton camera using camera lengths of 28.6 and $49.3 \mathrm{~mm}$ and nickel-filtered $\mathrm{CuK} \alpha$ radiation. Also, a Xentronics $\mathrm{X}-100 \mathrm{~A}$ system applying a multiwire proportional counter with monochromatic $\mathrm{CuK} \alpha$ provided azimuthal and radial traces of diffraction patterns presented later. Diffraction patterns were obtained with the incident beam along the $\mathrm{x}$ and $\mathrm{y}$ directions defined in Figure 2.2B. 
The Xentronics system digitally stores diffraction patterns using software packages for image processing. An Optronics 1500 digitized the diffraction patterns from the Statton camera to obtain azimuthal traces using software developed by Jack M. Gromek of the University of Connecticut.

Table 2.1

Description of PBZT/ Glass Samples

\begin{tabular}{|c|c|c|c|}
\hline & Heat Treatment & $\begin{array}{l}\text { Glass } \\
(\% \mathrm{w} / \mathrm{w})\end{array}$ & $\begin{array}{l}\text { Content } \\
(\% \mathrm{v} / \mathrm{v})^{*}\end{array}$ \\
\hline PBZT Control & Stage dried at $300^{\circ} \mathrm{C}$ & 0 & 0 \\
\hline $\mathrm{SiO}_{2}$ & Stage dried at $300^{\circ} \mathrm{C}$ & 35 & 30 \\
\hline $\mathrm{SiO}_{2}$ & $\begin{array}{l}\text { Stage dried at } 300^{\circ} \mathrm{C} \\
\text { Hot pressed at } 500^{\circ} \mathrm{C}\end{array}$ & 35 & 29.3 \\
\hline Borosilicate $\left(\mathrm{SiO}_{2} \cdot \mathrm{B}_{2} \mathrm{O}_{3}\right)$ & Stage dried at $300^{\circ} \mathrm{C}$ & 29 & 26.4 \\
\hline Borosilicate & $\begin{array}{l}\text { Stage dried at } 350^{\circ} \mathrm{C} \\
\text { Stage dried at } 500^{\circ} \mathrm{C}\end{array}$ & 29 & 24.1 \\
\hline Lead Borate $\left(\mathrm{PbO} \cdot \mathrm{B}_{2} \mathrm{O}_{3}\right)$ & Stage dried at $300^{\circ} \mathrm{C}$ & 29 & $24 * *$ \\
\hline $\begin{array}{l}\text { Lead Borate } \\
\text { (*Assuming specific gravities } \\
\text { [Kovar, 1987]) } \\
\text { (**Assuming specific gravity }\end{array}$ & $\begin{array}{l}\text { Hot pressed at } 300^{\circ} \mathrm{C} \\
\text { on neat glass samples } \mathrm{h} \\
\text { of } 2 \text { for the lead borate }\end{array}$ & $\begin{array}{c}29 \\
\text { d to } s i m i\end{array}$ & $\begin{array}{l}24 * * \\
\text { peratures } \\
g, 1937])\end{array}$ \\
\hline
\end{tabular}

\subsubsection{Microscopy}

A JEOL 35FX scanning electron microscope provided SEM images of the biaxial film samples. A gold coating on the specimens reduced charge build-up and the subsequent discharging to the detector. Polaroid P/N film was used to record the SEM images. 


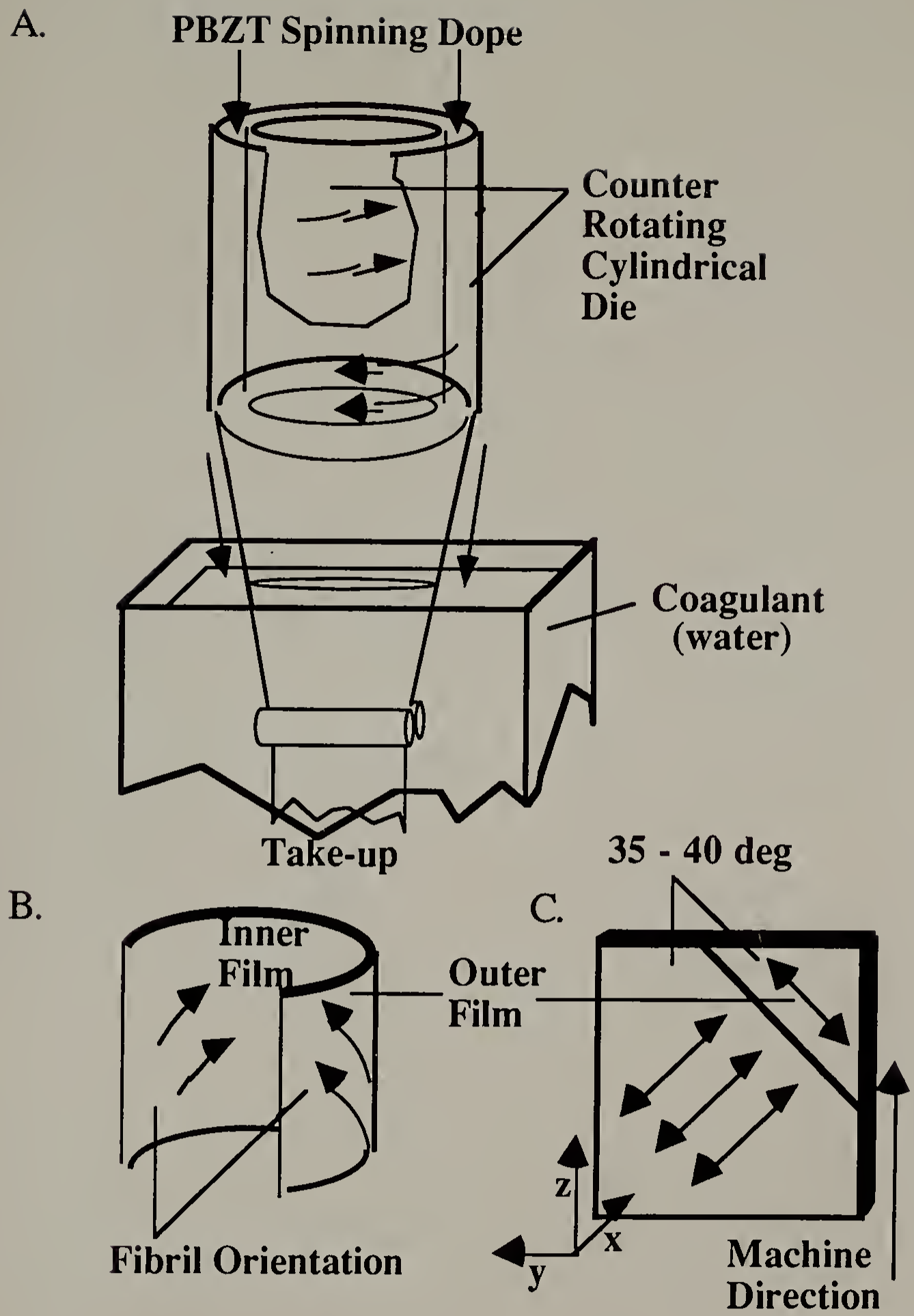

Figure 2.1 Schematic demonstrating (A) biaxial film production (B) the natural curl in film and $(C)$ the coordinate system of $X$-ray experiments. 


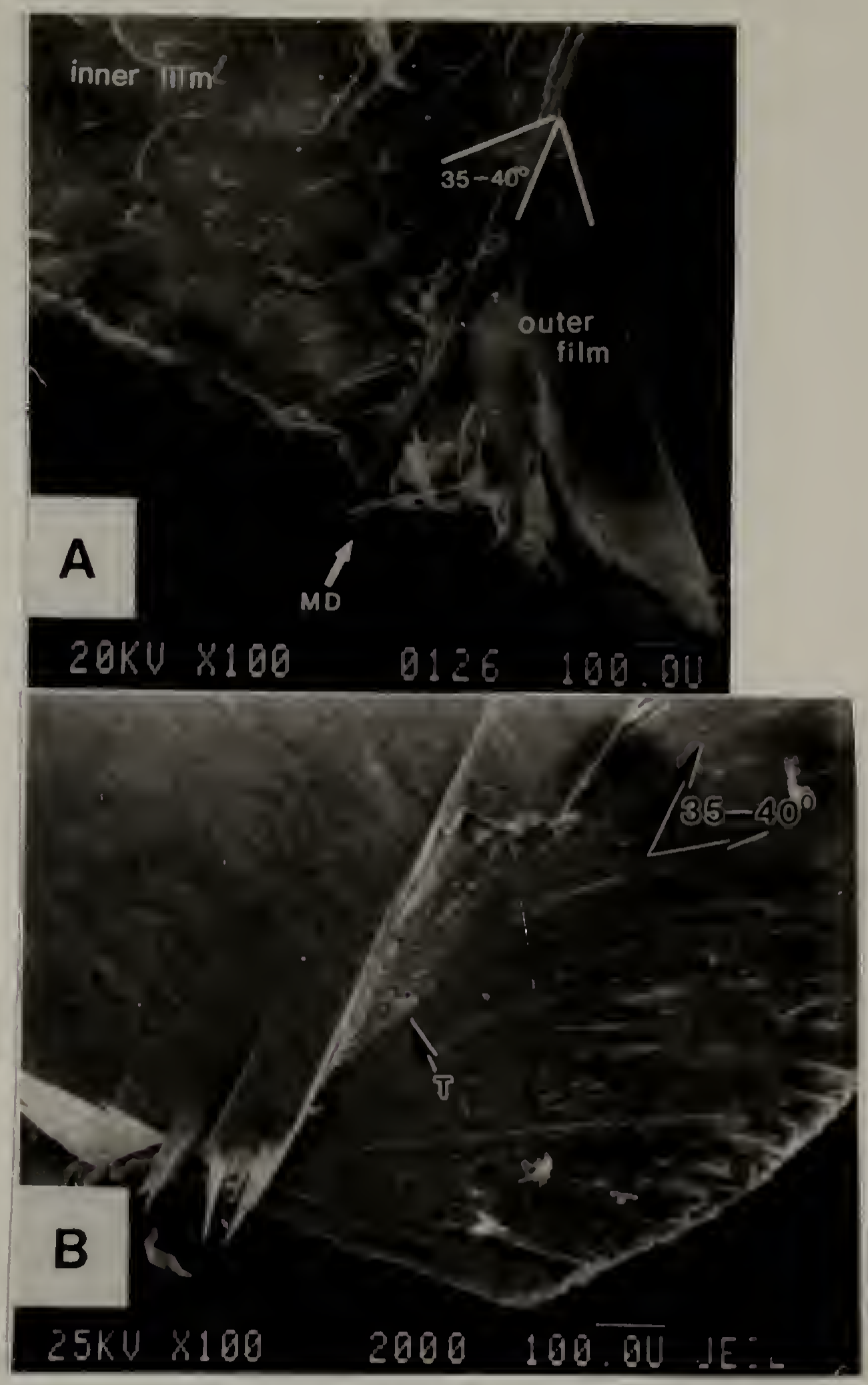

Figure 2.2 (A) SEM image of a cleaved PBZT biaxial film showing the two main layers into which the film readily divides. The fibrils tend to orient in two different directions in the two film halves. (B) SEM image of similar film in which the fracture surface indicates a twist in the fibril orientation through the film thickness. 


\section{PBZT Biaxial Film}

Layered Structure

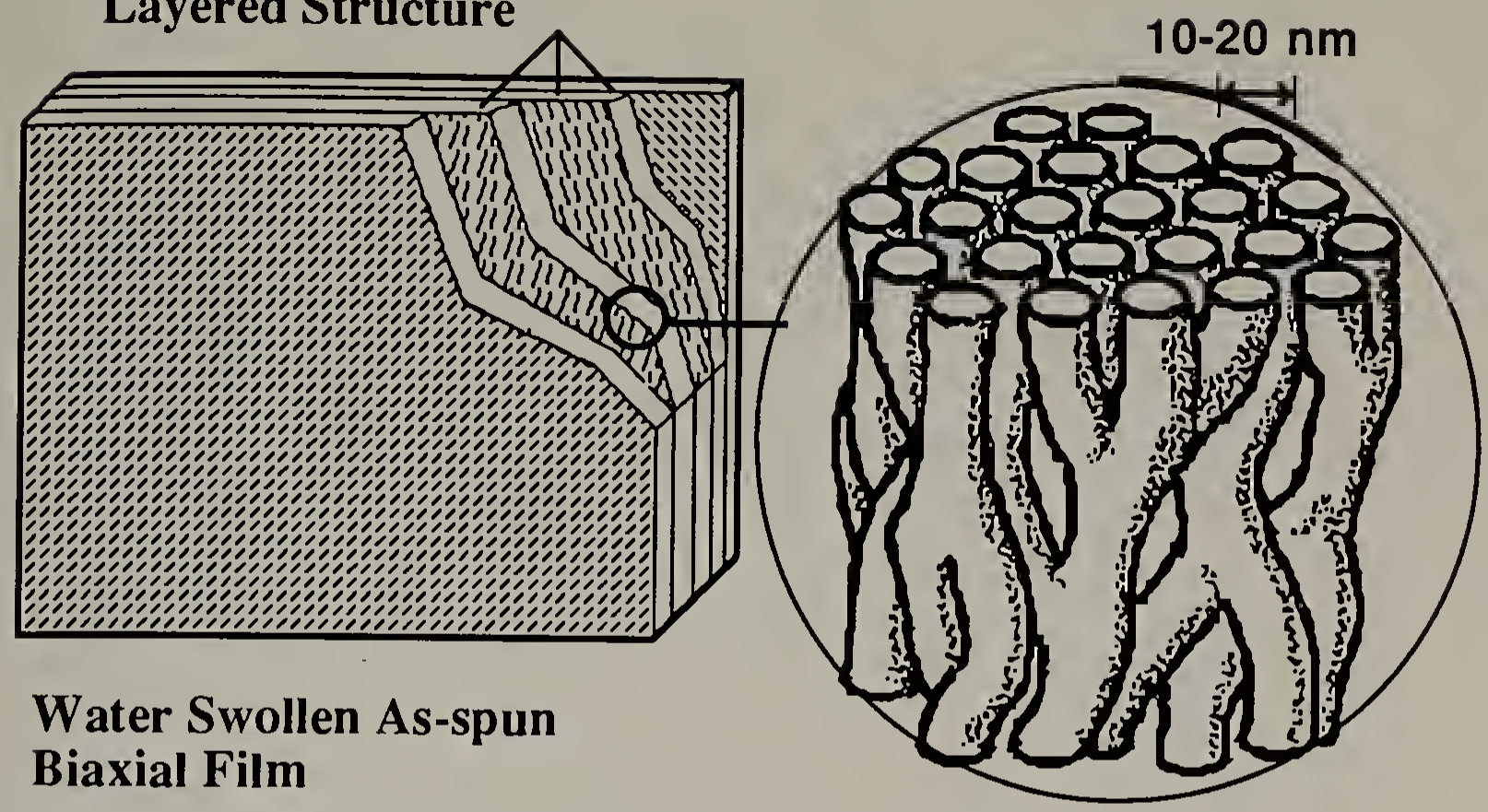

Sequential Replacement Of Water
With Water/Methanol Mixtures

Methanol Swollen Film

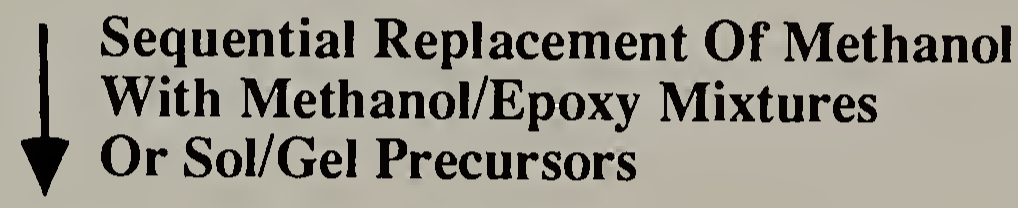

\section{Epoxy Or Sol/Gel Glass Swollen Film}

Figure 2.3 Schematic of infiltration procedure.

Pelco Medcast Quik-Mix Kit epoxy of medium hardness was the embedding medium for the film specimens. To facilitate microtomy, the films were mounted in a silicon rubber or polyethylene molds for cross and longitudinal sections. The epoxy was cured at $60^{\circ} \mathrm{C}$ for 16 to 20 hours. 
The embedded film samples were trimmed to approximately 1 square millimeter in size and sectioned a Sorvall MT2B ultra-microtome. A diamond knife will microtome PBZT films at ambient temperatures using water to float the sections after cutting. For TEM examination, copper grids supported the thin sections retrieved from the water surface.

TEM images of the film thin sections were obtained using a JEOL 100CX TEM or a JEOL 2000FX TEM at $200 \mathrm{kV}$ using Kodak SO-163 electron image film.

\subsection{WAXS and TEM Results}

\subsubsection{Stage drying}

Figure 2.4 shows X-ray diffraction patterns for the stage dried PBZT control film and various PBZT/sol-gel glass films after stage drying and other thermal treatments. Table 2.2 displays indexed crystalline reflections for samples with low diffuse scatter.

Stage-drying converts the sol/gel glass precursors into microvoid filled gels that diffusely scatter X-rays at low angles as shown in Figures 2.4 B, D, and F. The microvoid scattering does not have a preferred orientation and its presence does not appear to alter the symmetry of the PBZT reflections. The microvoid scattering obscures the inner [001] reflections; therefore, some of the voids can be estimated to be 1.2 nanometers in size. The lead borate glass infiltrated sample scatters the most of the stage dried samples due to the larger electron density difference from the presence of the $\mathrm{Pb}$ atoms.

The X-ray diffraction patterns of the glass impregnated films show strong diffuse scattering only before heat treatment; (note that heat treatment means higher temperatures than normal stage drying). The stage dried PBZT/lead borate composite film shows two diffuse wide angle halos corresponding to an average spacings of 1.0 nanometers and 3 
to 4 nanometers, indicative of an amorphous glass. Strong diffuse scattering is also present at low angles from larger ( $>5.0$ nanometers) microvoids.

\subsubsection{Annealing effect on X-ray scattering}

Figure 2.4 also illustrates the effect of the glass fusion upon the presence of voids. For the glass samples with varying heat treatments, the glass increases in density as microvoids disappear in the coagulated, dried gel thereby reducing the observed diffuse scattering. The densification heat treatment significantly reduces the diffuse scatter of the glass impregnant as can be seen by comparison of the intensity of the diffuse scattering with that of the PBZT reflections.

Figures $2.4 \mathrm{C}$ and $2.4 \mathrm{E}$ show the $\mathrm{X}$-ray diffraction patterns of the heat treated PBZT films infiltrated with silicate and borosilicate glass, respectively. The patterns do not differ from the PBZT control in Figure 2.4A as the spacing measurements in Table 2.2 attest.

Table 2.3 shows the indexes of the reflections of the diffraction pattern in Figure 2.4G. Besides an observed reduction in diffuse scatter upon heat treatment, the lead borate sol-gel impregnant crystallizes with isotropic orientation. The hot pressed PBZT/lead borate glass composite film exhibits a number of new reflections that are characteristic of unoriented crystallized lead borate glass [McMurdie and Bunting, 1939, Krough-Moe and Wold-Hansen, 1973, Perloff and Block, 1966, Fieser and Goldsmith, 1978]. The observed reflections qualitatively fit with the calculated reflections for the compounds of, hexalead pentaborate $\left(6 \mathrm{PbO} \cdot 5 \mathrm{~B}_{2} \mathrm{O}_{3}\right)$ [Krough-Moe and Wold-Hansen, 1973] lead tetraborate $\left(\mathrm{PbO}^{2} \mathrm{~B}_{2} \mathrm{O}_{3}\right)$ [Perloff and Block, 1966] and crystalline elemental $\mathrm{Pb}$. Table 2.3 shows the correspondence of the measured reflections to those calculated from the given unit cell parameters of $\mathrm{PBZT}, 6 \mathrm{PbO} \cdot 5 \mathrm{~B}_{2} \mathrm{O}_{3}, \mathrm{PbO} \cdot 2 \mathrm{~B}_{2} \mathrm{O}_{3}$, and $\mathrm{Pb}$. The lack of identification of all reflections shows the difficulty of classifying components in 
crystallizing lead glasses [Krough-Moe and Wold-Hansen, 1973]. The correlation with the reflections for the lead tetraborate does not appear to match as well at the higher $d$ spacings as for the reflections for the hexalead pentaborate.

The data indicates hot pressing greatly affects the form of the lead borate glass. However, it is not clear whether the crystallization is a result of the applied pressure or the additional time the hot pressed sample is at elevated temperature compared to the stage dried sample.

\subsubsection{Orientation Distribution Estimation}

As previously noted, the balanced biaxial PBZT films have a layered fibrillar structure [Lusignea, 1989]. Figure 2.2A illustrates that the films easily split into two layers with the fibrils having distinct orientations inclined 35 to 40 degrees from the machine direction. However, Figure 2.2B shows a progression of twist of adjacent layers that suggest a distribution of orientations. Figures 2.5 and 2.8 show comparisons of X-ray diffraction patterns the inner and outer film portions for the neat and impregnated PBZT films, respectively, along with the scattering patterns for whole films. After digitizing the diffraction patterns, the data analysis entails integrating the intensity with respect to the scattering angle, $\theta$, over a defined annular region, ( $r_{1}$ to $\left.r_{2}\right)$, and plotting the results versus the azimuthal angle, $\phi$. The schematic in Figure 2.6 illustrates the procedure; Figures 2.7 and 2.9 show plots of relative intensity versus $\phi$ the PBZT and PBZT/borosilicate films.

If the fiber axis is perpendicular to the incident beam, the variation of intensity with azimuthal angle, $\phi$, relates to both the Bragg scattering angle of a reflection, $\theta$, and the orientation of the crystallographic planes relative to the fiber axis, $\beta$, by the following equation:

$$
\cos \beta=\frac{\cos \phi}{\cos \theta}
$$


This relation holds for meridional reflections and applies to equatorial reflections if the $\mathrm{a}$ and $\mathrm{b}$ axes of the unit cell are perpendicular to the $\mathrm{c}$ axis. Since $\theta$ is small and provided $\beta>>\theta$, then the intensity at $\phi$ is equivalent to the density of crystallographic planes oriented at the angle $\beta$ with respect to the fiber axis [Alexander, 1969]. The equatorial reflections for the [010] are used in our orientation measurements because the meridional reflections are weak and the axial disorder in PBZT causes strong streaking of these meridional diffraction signals.

\subsubsection{Orientation Function Estimation}

Comparison of azimuthal intensity scans shows that the orientation distribution in the balanced biaxial film samples does not exhibit cylindrical (fiber) symmetry. However, assuming fiber symmetry, calculated orientation function values can give a relative basis for comparison of a series of related samples. Absolute fiber orientation function calculations require knowledge of the $\beta$, the angle of the crystalline planes with respect to the reference axis.

Table 2.4 lists the orientation function values determined from azimuthal scans of the [010] equatorial reflection with respect the machine direction, the $\mathrm{z}$ axis. A value of zero indicates no preferred orientation (isotropic) and a negative value denotes preferred perpendicular alignment with a value of -0.5 indicating perfect perpendicular orientation.

The whole film exhibits on average almost planar isotropic molecular orientation, which correlates well with the physical property data. [Lusignea, 1989]. The average molecular alignment in the film plane is higher in the inner film than the outer film; the outer half probably contains the region of twist shown in Figure 2.2B. The distribution function value shows that the outer film shows almost isotropic alignment.

Glass impregnation does not significantly affect the molecular orientation in the films. Coagulation and the subsequent heat treatment reduce the voids in the dried gel, 
but do not cause any significant difference in the observed average PBZT molecular orientation.

Figure 2.11 shows an $\mathrm{X}$-ray diffraction pattern for a PBZT film with the $\mathrm{X}$-ray beam incident from the $\mathrm{y}$ direction. An average of the intensities can be obtained from scattering patterns with the $\mathrm{X}$-ray beam incident from the $\mathrm{x}$ and $\mathrm{y}$ directions to yield a refined estimation of the axial symmetric orientation function for these films. The molecular alignment through the film thickness shows high orientation with respect to the machine direction.

Figure 2.13 shows a suggested model for the molecular orientation with respect to the film thickness based upon the SEM images and the X-ray diffraction data. The data shows that the a major portion of the material is oriented at an angle determined by the counter rotating speed and the throughput. There is a gradual twist in orientations nearer the film surfaces. Near the center of the film there is a large change in orientation direction in a shorter distance. 
Figure 2.4 PBZT film $X$-ray diffraction patterns with the $X$-ray beam incident from the $X$-direction. (A) Control (No glass), stage dried at $300^{\circ} \mathrm{C}$. (B) Silicate, Stage dried at $350^{\circ} \mathrm{C}$. (C) Silicate, Hot Pressed at $500^{\circ} \mathrm{C}$. (D) Sodium Borosilicate Stage dried at $350^{\circ} \mathrm{C}$. (E) Sodium Borosilicate Hot Pressed at $500^{\circ} \mathrm{C}$. (G) Lead Borate Stage at $300^{\circ} \mathrm{C}$. (H)Lead Borate, Heat treated at $300^{\circ} \mathrm{C}$. 


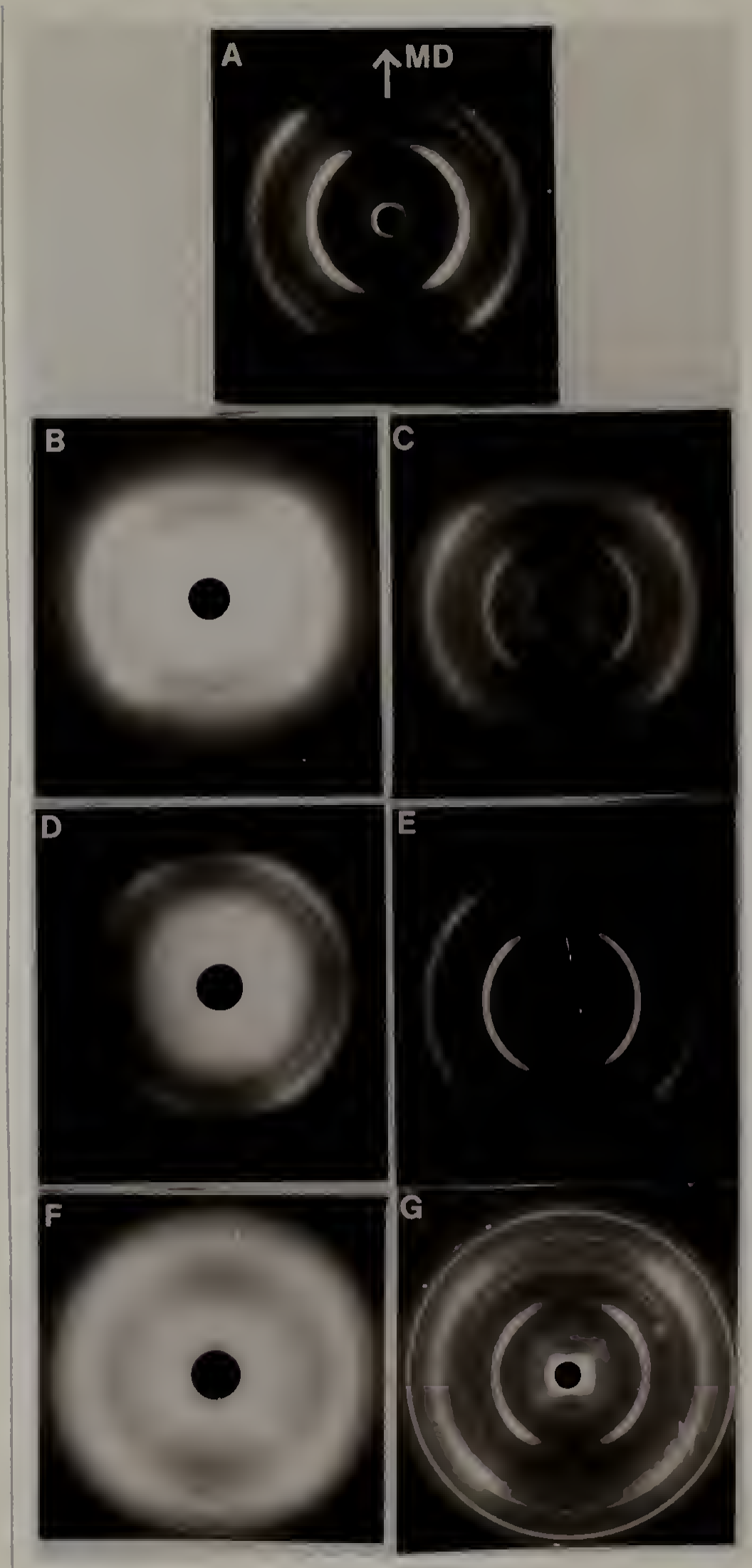


PBZT Unit Cell: $a=0.597 \mathrm{~nm} \quad b=0.362 \mathrm{~nm} \quad c=1.245 \mathrm{~nm} \quad \gamma=95.2^{\circ}$

SAMPLE

[Lattice Plane]

Spacings (nm)

Control

[001] [002] [003] [110] $\begin{array}{ll}{[100]} & {[010] \quad[?] \quad \phi} \\ \text { and }\end{array}$ [002] (deg)

$\mathrm{SiO}_{2}$

$1.24 \quad 0.62$

0.41

0.59

0.37

33

$\begin{array}{lll}1.24 & 0.62 \quad 0.42\end{array}$

0.59

0.37

36

Borosilicate

$1.24 \quad 0.62$

0.41

0.60

0.36

5*

33

Borosilicat

1.24

0.41

$0.59 \quad 0.36$

*very weak reflection observed on $24 \mathrm{~h}$ exposure 
Table 2.3 Indexing of Hot Pressed PBZT/Lead Borate Composite Film

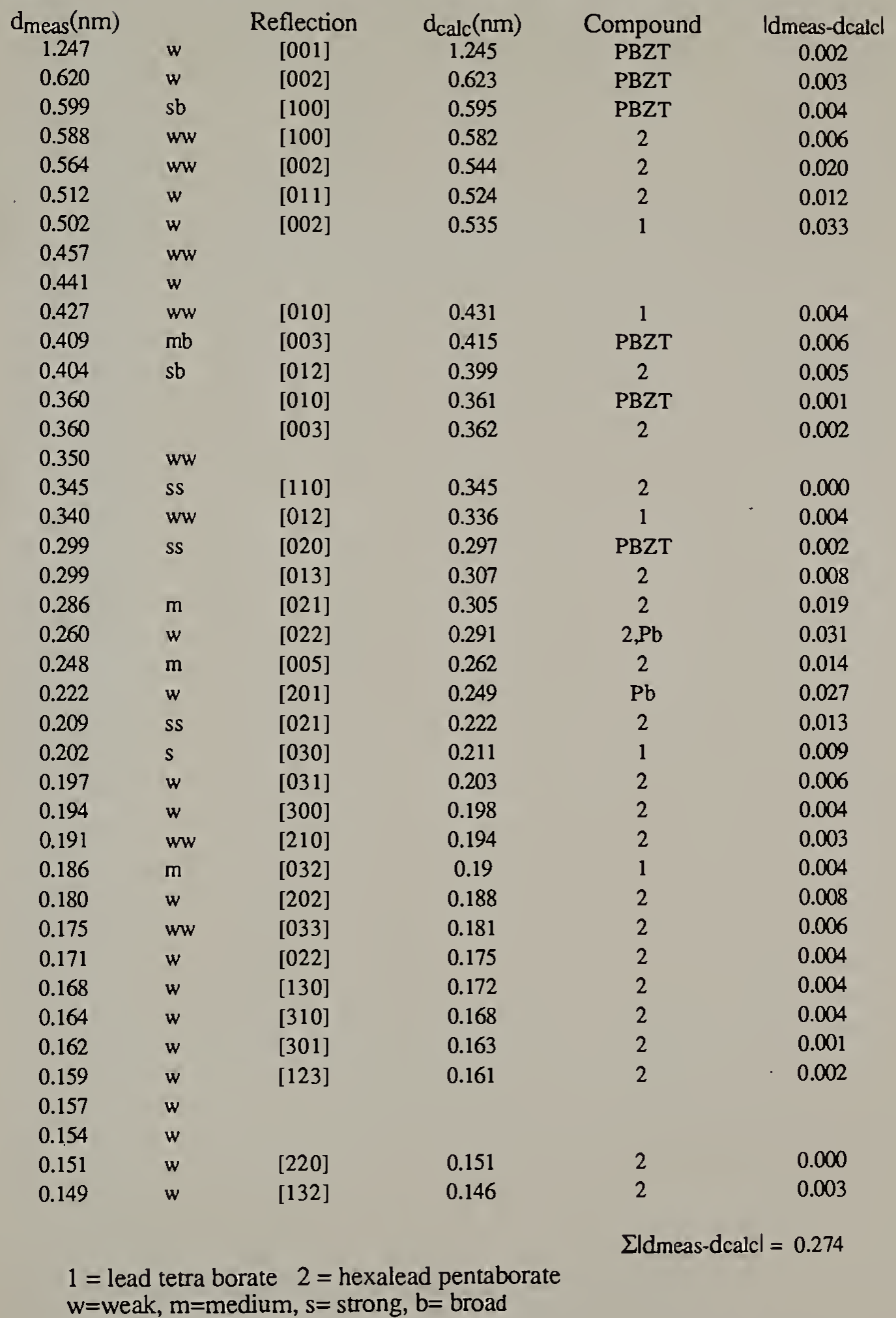




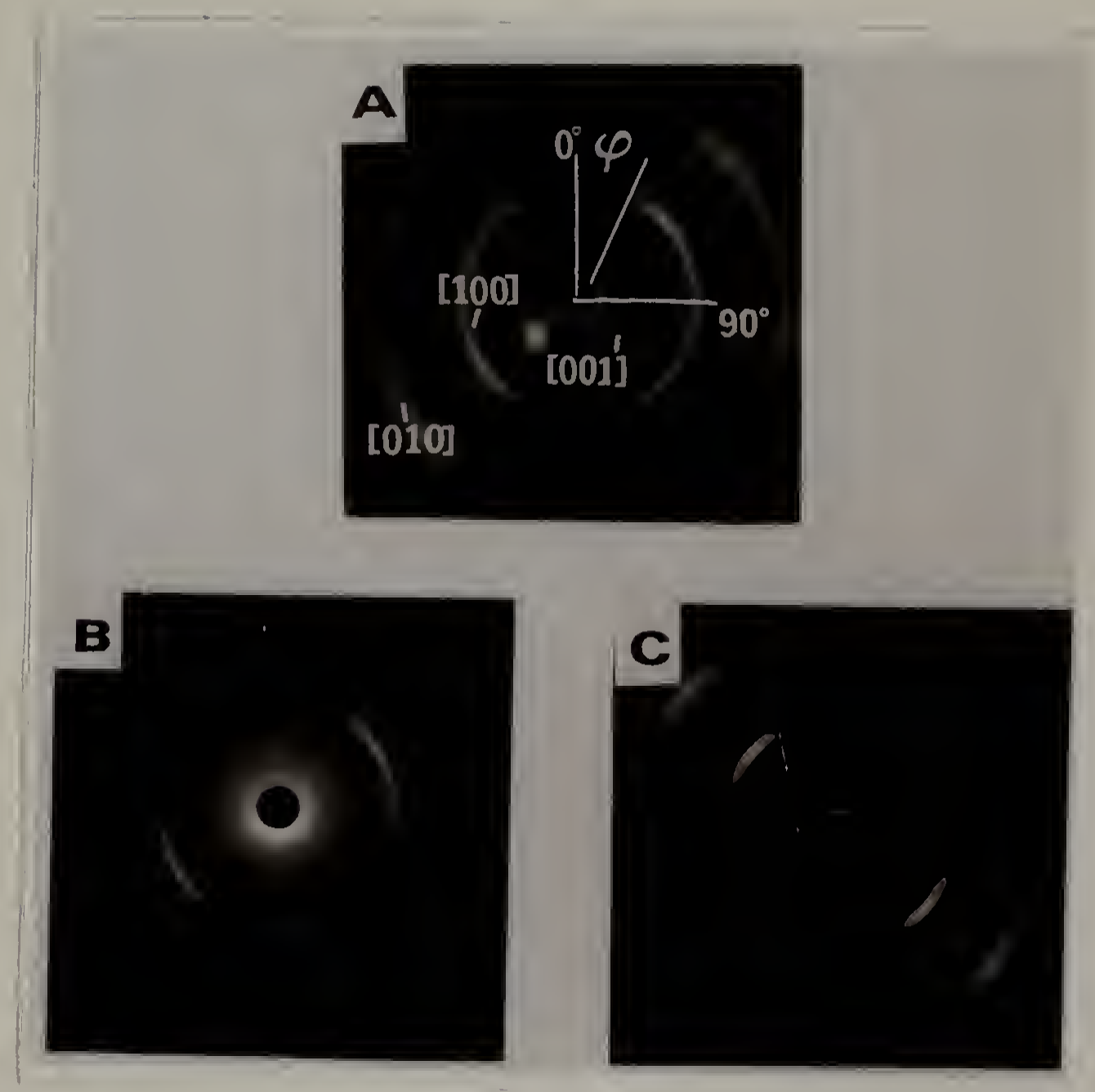

Figure 2.5 X-ray diffraction patterns with beam incident from the $\mathrm{x}$-direction of PBZT film (A) that splits into two layers; an outer layer (B) and an inner layer $(C)$. $\phi$ is the azimuthal angle with the $\mathrm{z}$ axis shown in Figure 2.1. 


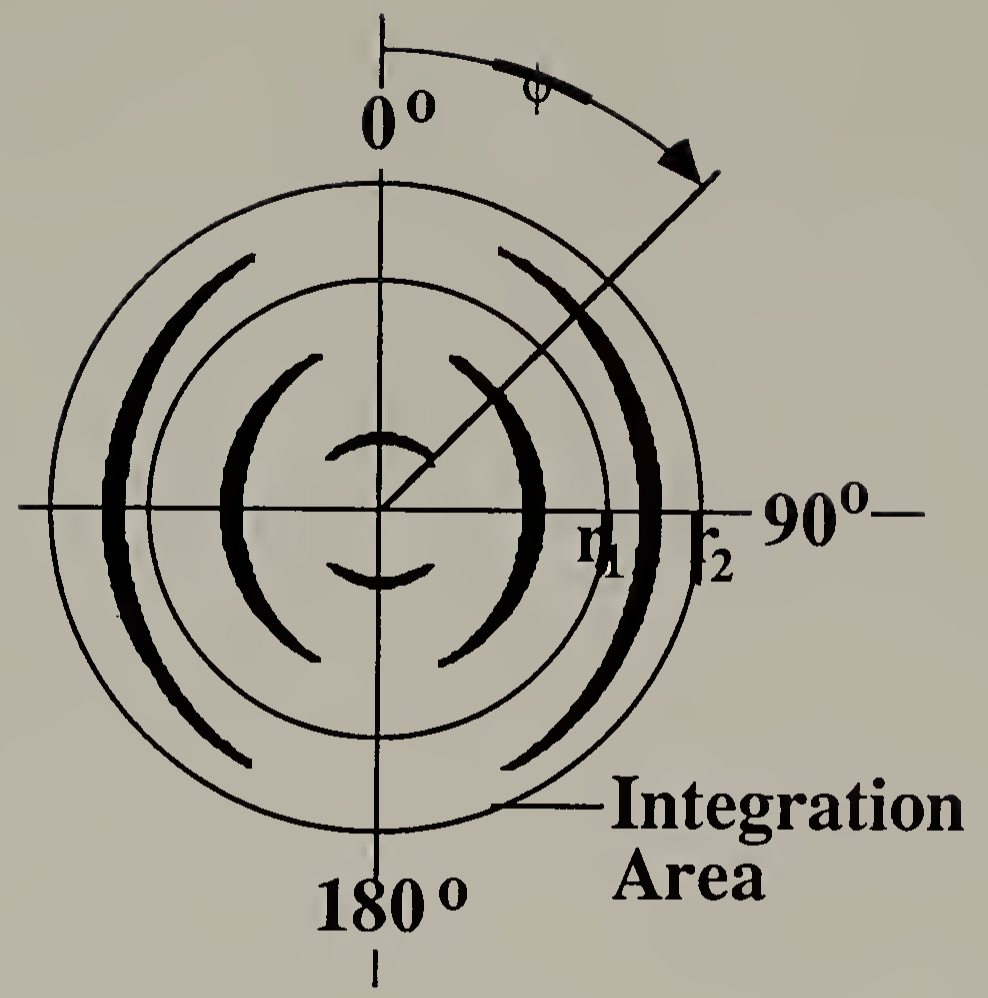

$\int_{\mathrm{r} 1}^{2} \mathrm{I}(\phi, \mathrm{r}) \mathrm{dr}$ plotted versus $\phi$

Figure 2.6 The schematic demonstrates the method of obtaining integrated intensity versus azimuthal angle plots from the $\mathrm{X}$-ray diffraction patterns. 
A.
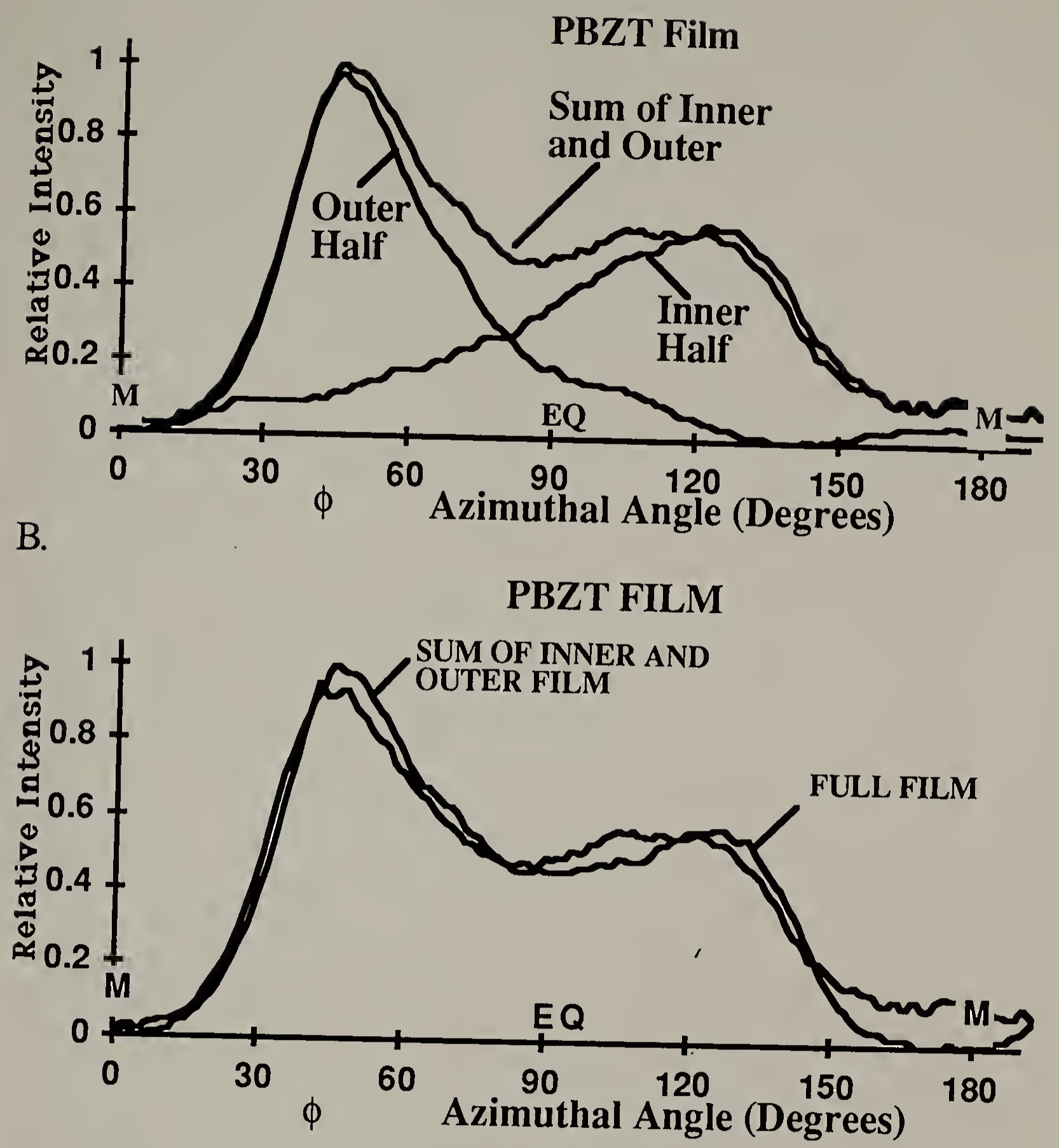

Figure 2.7 (A.) A trace of relative integrated intensity verses the azimuthal angle, $\phi$, for the $X$-ray patterns in Figure 2.5. The intensities of the two film halves were weighted so their sum will yield the trace for the full film. (B.) A comparison of the sum from Figure 2.7A and the relative intensity versus azimuthal angle, $\phi$, for the whole PBZT film. 


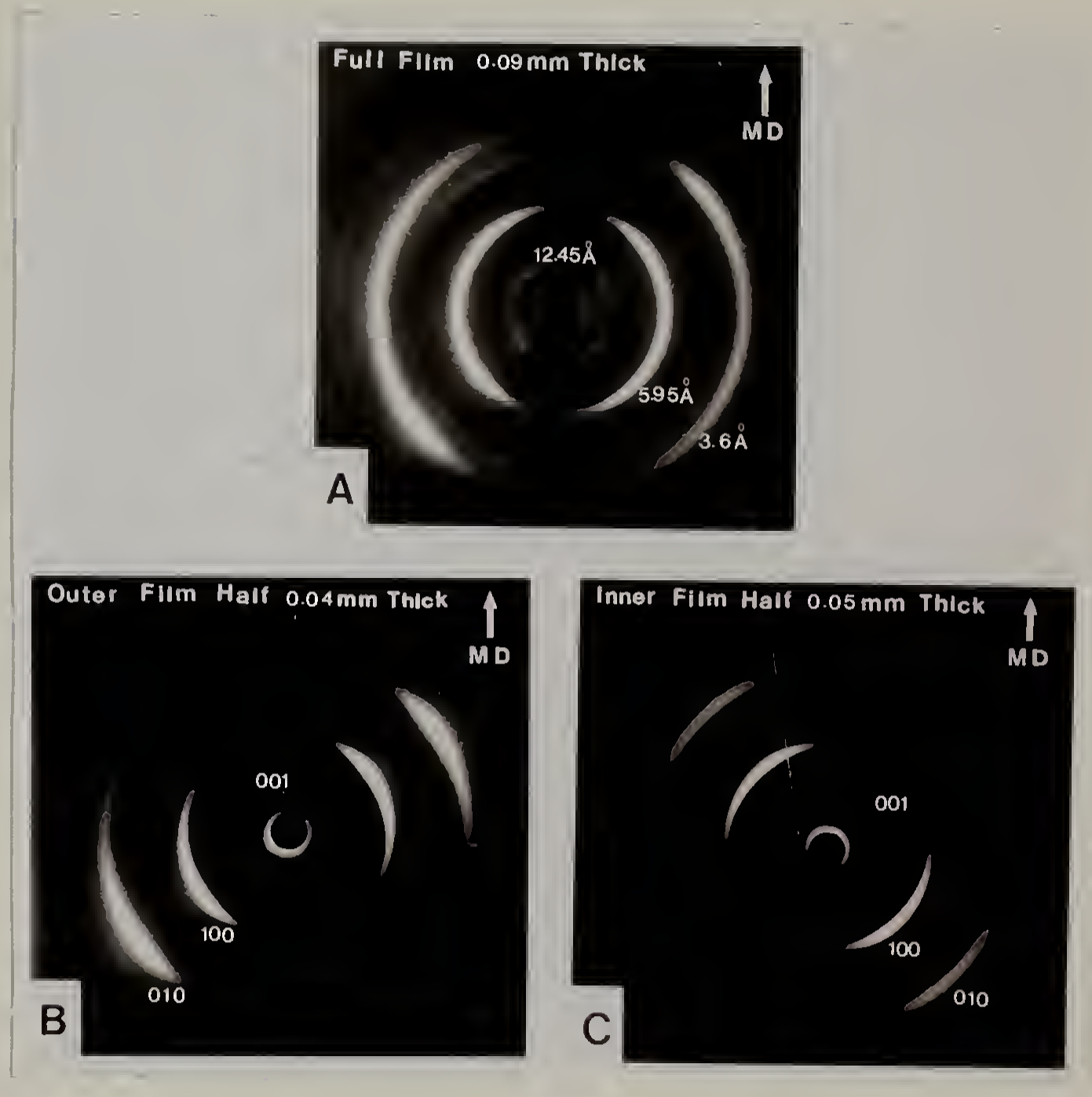

Figure 2.8 X-ray diffraction patterns from a heat treated PBZT/borosilicate glass composite film (A) split into two layers; an outer layer (B) and an inner layer $(C) . \phi$ is the azimuthal angle with the $\mathrm{z}$ axis and the molecular orientation. 

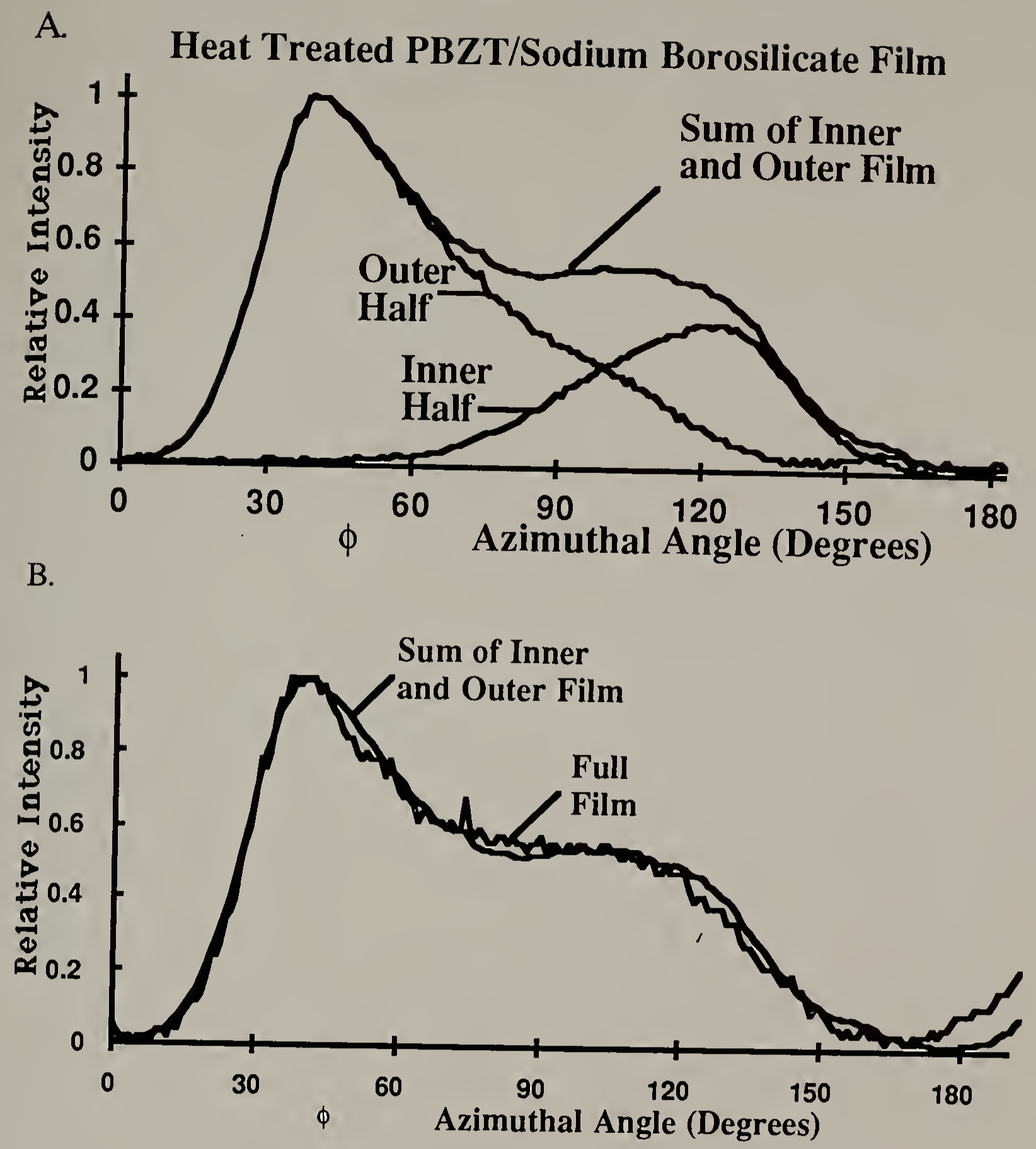

Figure 2.9 (A.) A trace of relative integrated intensity verses the azimuthal angle, $\phi$, for the $X$-ray patterns in Figure 2.8. The intensities of the two film halves were weighted so their sum will yield the trace for the full film. (B.) A comparison of the sum from Figure 2.9A and the relative intensity versus azimuthal angle, $\phi$, for the whole PBZT film. 


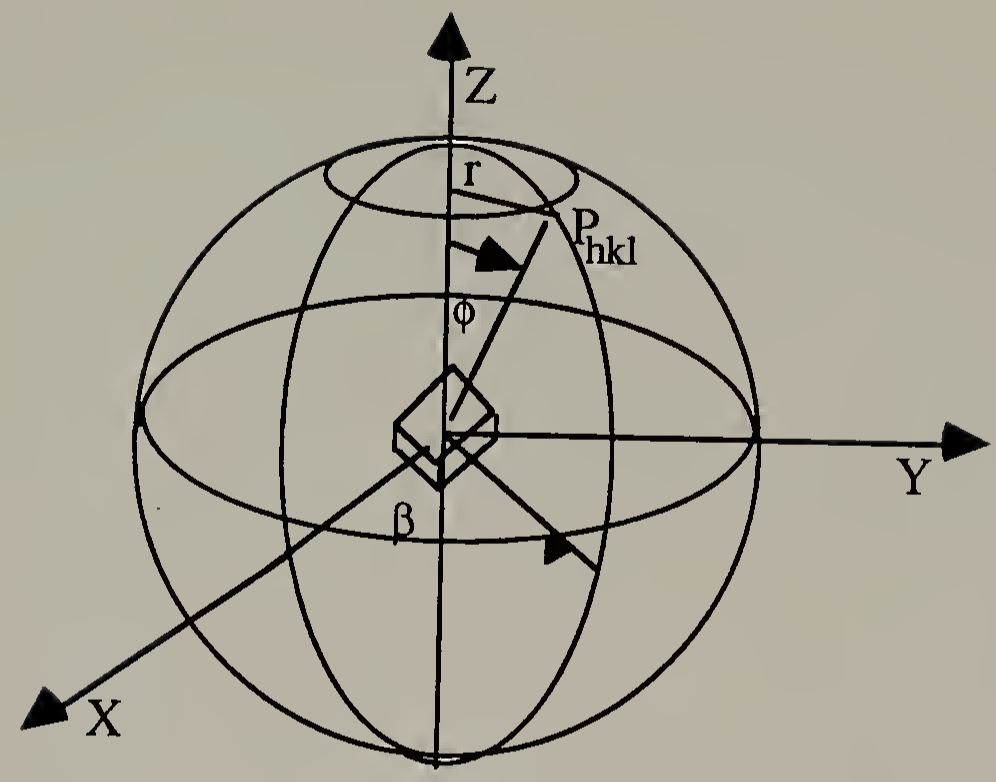
Figure 2.10 A schematic showing the relationship between $\phi, \beta$ and $\mathrm{r}(2 \theta)$ [Alexander,
1969]. 


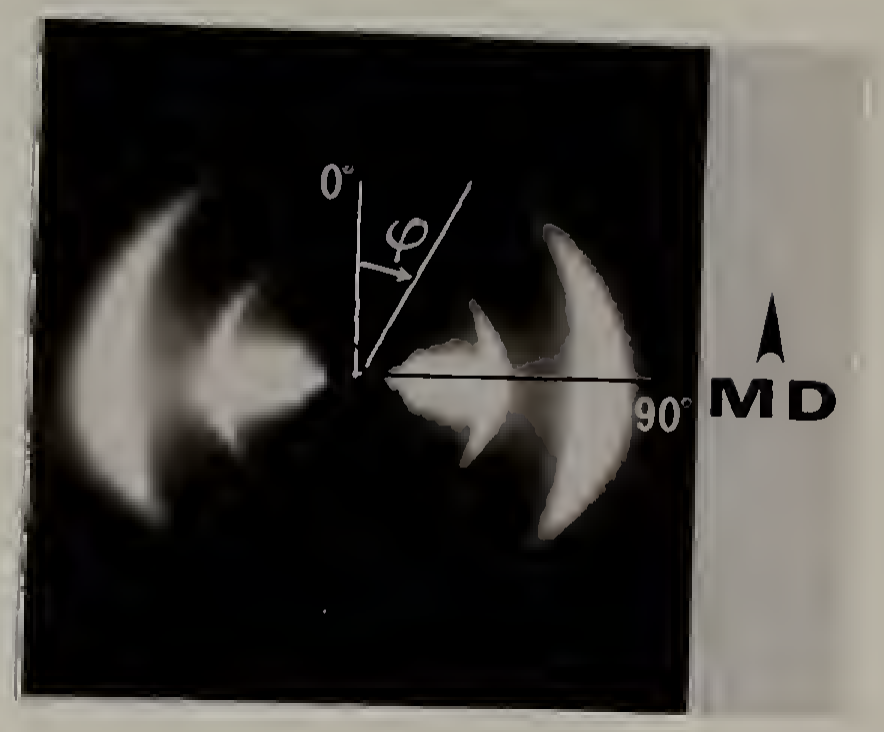

Figure 2.11 X-ray diffraction patterns from folded biaxially oriented PBZT/borosilicate film. The X-ray beam is incident from the $y$ direction. The [010] reflection has a much higher intensity due to the crystallite orientation within the $y-z$ plane. 

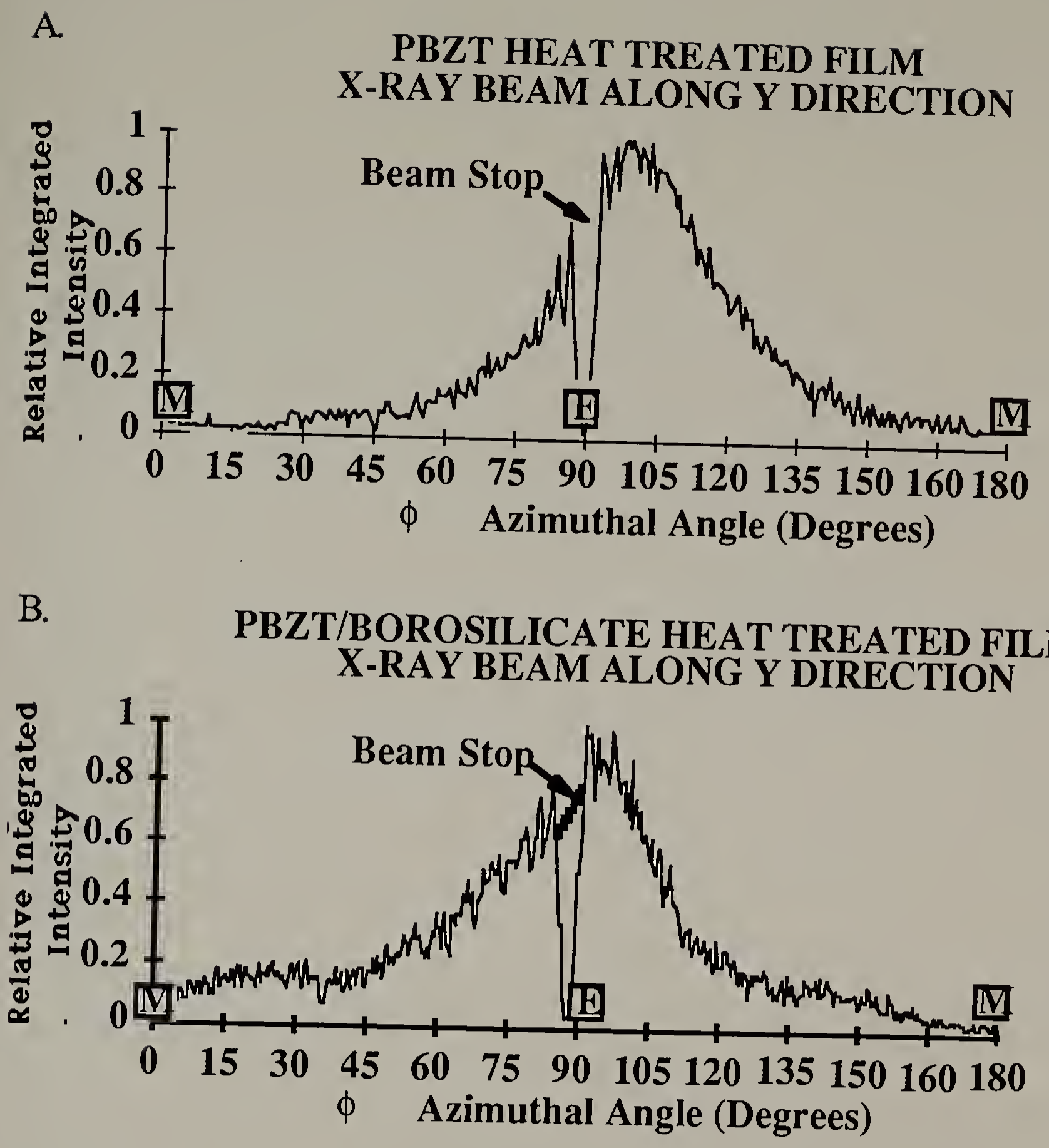

Figure 2.12 A trace of relative integrated intensity verses the azimuthal angle, $\phi$, for (A) a folded PBZT film and (B) a folded PBZT/borosilicate in which the $\mathrm{X}$-ray beam is incident in the $\mathrm{y}$ direction (see Figure 2.1C). 
Table 2.4

Orientation Function Values for Cylindrical Symmetry

$$
\begin{gathered}
\mathrm{f}=\frac{1}{2}\left(3<\cos ^{2} \phi h k \mathrm{z}>-1\right)=\frac{\int_{0}^{\pi} \mathrm{I}(\phi) \sin \phi \cos ^{2} \phi d \phi}{\int_{0}^{\pi} \mathrm{I}(\phi) \sin \phi \mathrm{d} \phi} \\
\mathrm{I}(\phi)=\int_{0}^{\pi} \mathrm{I}(\phi, \beta) \mathrm{d} \beta
\end{gathered}
$$

$\mathrm{X}$-ray Beam Direction: $\quad \mathrm{x}$ axis

$\mathrm{y}$ axis

$\mathrm{x}$ and $\mathrm{y}$ axes

Sample:

$$
\mathrm{I}(\phi, \beta)=
$$

$\mathrm{I}(\phi, 0)$

$\mathrm{I}(\phi, 90)$

$(\mathrm{I}(\phi, 0)+\mathrm{I}(\phi, 90)) / 2$

PBZT Film

PBZT Film inner

PBZT Film outer

PBZT/Borosilicate Film

PBZT/Bs Film inner

PBZT/Bs Film outer
$-0.08$

$-0.26$

$-0.15$

$-0.13$

$-0.03$

$-0.09$

$-0.25$

$-0.15$

$-0.17$

$-0.04$ 


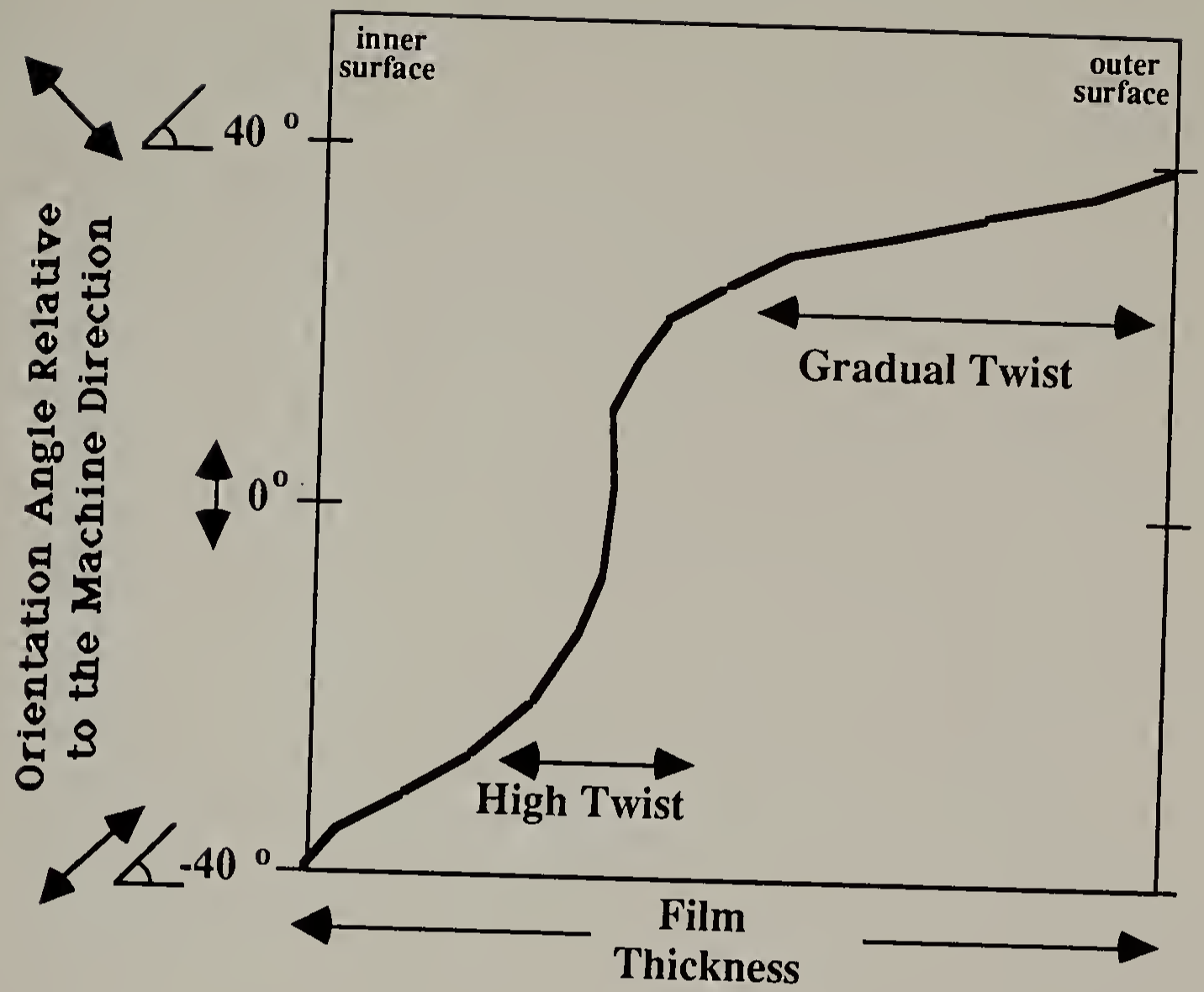

Figure 2.13 A suggested model for the through thickness orientation distribution based upon SEM and X-ray diffraction evidence. 


\subsubsection{Crystallite Orientation and Size}

The data analysis involves integrating intensity $\mathrm{I}(\phi)$ with respect to the azimuthal angle, $\phi$, and then plotting versus the scattering angle, $2 \theta$ (or radial distance $r$ on the scattering pattern).

The expression

$$
\int_{0}^{2 \pi} I(\phi, 2 \theta) d \phi
$$

is plotted versus $2 \theta$. The variable $r$ is the scattering angle $2 \theta$ (which can be represented as the radial distance on the scattering pattern, see Figure 2.14).

Azimuthally averaged radial intensity profiles of $X$-ray scattering patterns can give indications on how the chains are oriented about the machine direction. In Figure 2.15 the azimuthal averaged radial intensity of the [100] crystal plane reflection is higher relative to the [010] reflection when the $\mathrm{X}$-ray beam is incident on the film in the $\mathrm{x}$ direction than when the beam is along the $y$ direction. For uniaxial fiber symmetry, the intensity of the [010] reflection would be a constant percentage of the [100] reflection intensity independent of the radial $\mathrm{X}$-ray beam direction. Figure 2.15 indicates that the [100] crystal planes are oriented preferentially in the plane of the film [Minter, 1982].

The data indicates across the thickness of the film the PBZT molecules tend to preferentially align with the face of the aromatic rings in the plane of the film. This molecular orientation may be an important factor for the laminated sheet morphology observed in these biaxially oriented films.

The crystallite size can also be evaluated from the breadth of the peaks in Figure 15 [Minter, 1982 and Hermans et al., 1946] using the Scherrer equation,

$$
\mathrm{L}_{\mathrm{hkl}}=\frac{\mathrm{K} \lambda}{\beta_{0} \cos \theta}
$$


where Lhkl is the mean dimension of the crystallite perpendicular to the hkl planes, $\beta_{0}$ is the breadth at half-maximum of the reflection profile in radians, and $\mathrm{K}$ is a constant usually assigned the value of one. The data was not corrected for instrumental broadening so the values are lower bounds to the lateral crystal size. The results listed in Table 2.5 (1.5-3.0 nanometers) are comparable to previous calculations by Minter for Xray data of PBZT uniaxial films [Minter, 1982].

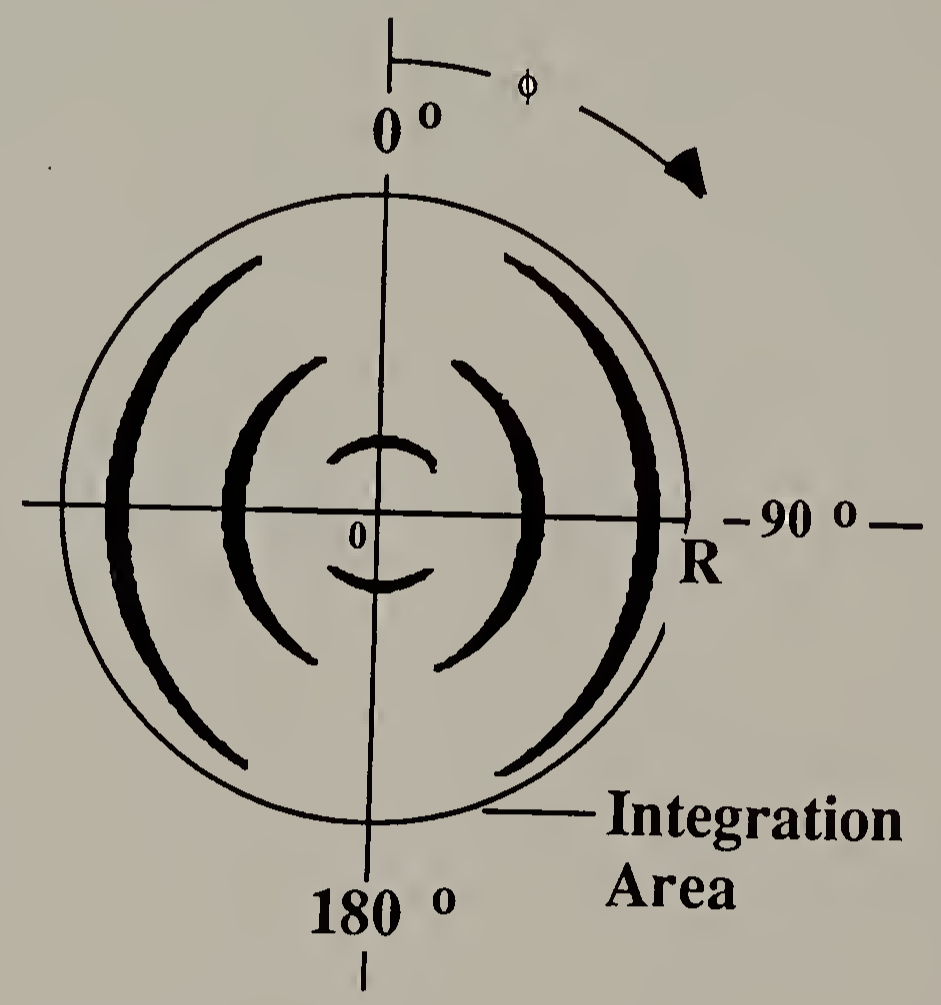

$$
\int_{0}^{2 \pi} I(\phi, r) d r \text { plotted versus } 2 \theta
$$

Figure 2.14 The method of acquiring of the relative azimuthally integrated intensity versus scattering angle, $2 \theta$, from $X$-ray diffraction patterns. 


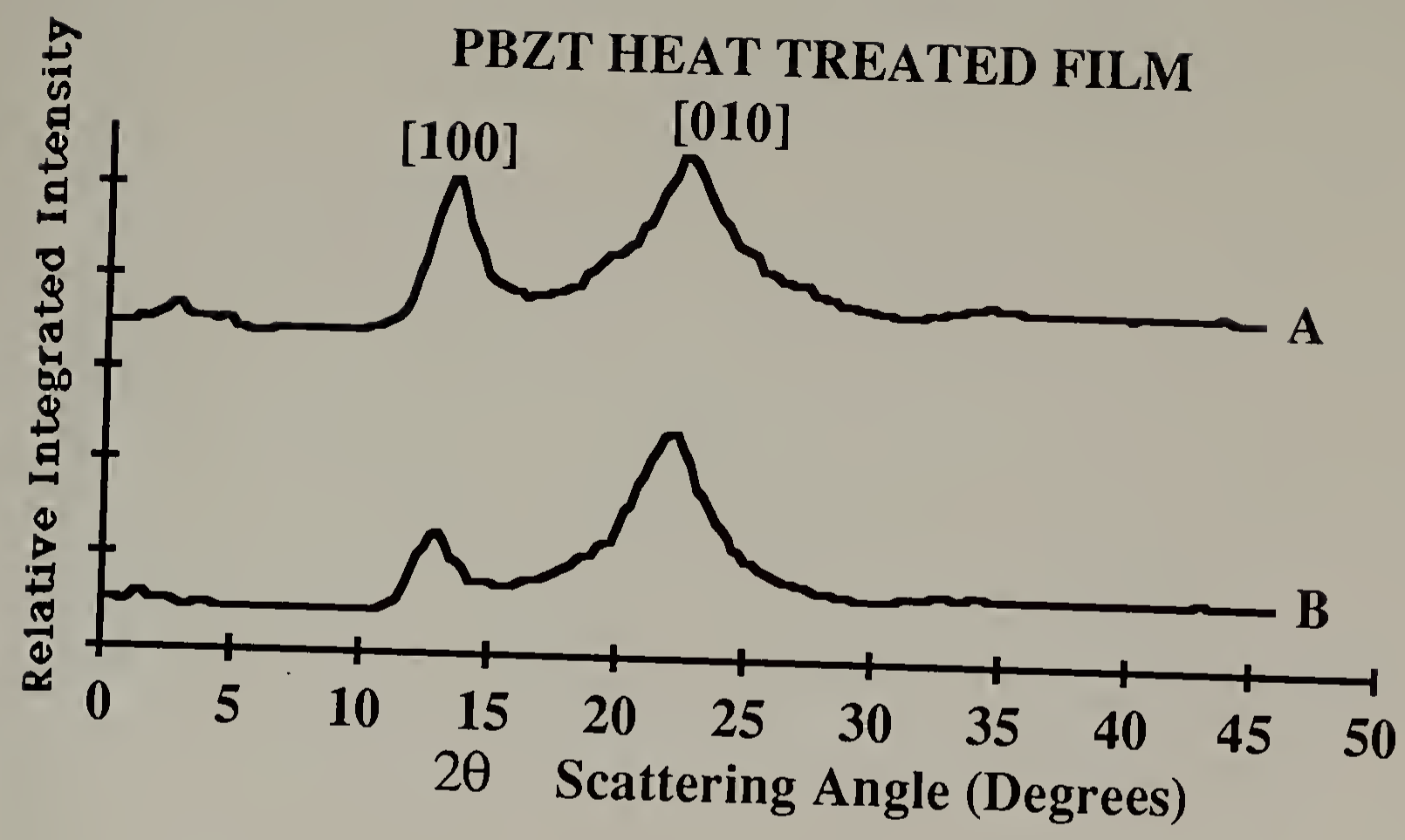

Figure 2.15 A comparison of azimuthally integrated intensity versus scattering angle for PBZT films with the X-ray incident from $(\mathrm{A})$ the $\mathrm{x}$ direction and $(\mathrm{B})$ the $y$ direction. Crystallite size estimates are given in Table 2.5 .

Table 2.5

Analysis of X-ray Data

$\begin{array}{lcccc} & \begin{array}{c}\text { X-Ray Beam } \\ \text { Direction }\end{array} & \begin{array}{c}\text { Crystal Size Calculation }\left(\mathrm{L}_{\mathrm{hkl}}\right) \\ {[100]}\end{array} & \begin{array}{c}\text { Intensity Ratio } \\ {[010]}\end{array} & \mathrm{I}_{[100]} \mathrm{r}_{[100]^{2}} \\ \text { PBZT Film } & \mathrm{Y} \text { (Edge on) } & 2.2 & 1.6 & 1.17 \\ \text { Full** } & \mathrm{X} & 2.4 & 1.5 & 2.31 \\ \text { Full** } & \mathrm{X} & 2.3 & 2.8 & 4.06 \\ \text { Full } & \mathrm{X} & 2.3 & 2.4 & 5.67 \\ \text { Inner half } & \mathrm{X} & 2.3 & 2.5 & 4.12 \\ \text { Outer half } & \mathrm{Y} \text { (Edge on) } & 1.8 & 1.4 & 2.31 \\ \text { Full } & & & & \end{array}$

PBZT/BS

Film

Full

Inner half

Outer half

Full

$\underset{X}{X}$

2.7

2.7

3.4

$\mathrm{Y}$ (Edge on)

1.8

* See Figure 2.2 for coordinate system

**Obtained on Xentronics System

$\begin{array}{ll}2.8 & 4.00 \\ 2.9 & 5.79 \\ 2.3 & 4.53 \\ 1.9 & 2.72\end{array}$




\subsubsection{TEM Images}

Figure 2.16 shows a schematic depicting arrangement of film samples for microtoming. Figure 2.17 compares TEM bright and dark field micrographs of cross sections of a PBZT stage dried control (no glass) film with both stage dried and heat pressed PBZT/lead borate films. A layered structure in the images follows a direction independent of the knife marks. However, selective electron diffraction measurements indicate that the orientation of the PBZT is in the knife direction due to deformation during microtoming [Cohen, 1987].

The microtoming process enhances visualization of the layers by applying compressive stresses that separate the microfibrils. The observed layers in the PBZT control film have a thickness on the order of 50 to 100 nanometers.

In Figures $2.17 \mathrm{~B}$ and $2.17 \mathrm{E}$, cross sections of the stage dried only lead borate sample show the same layered PBZT film structure. The lead borate glass appears as the large dark objects in the bright field images. These discrete particles are about 100 nanometers in diameter and are somewhat elongated along the knife direction.

The TEM bright and dark field images in Figures $2.17 \mathrm{C}$ and $2.17 \mathrm{~F}$ show a cross section of the hot pressed PBZT/lead borate sample. Many smaller particles are resolvable in the TEM micrographs of a cross section of hot pressed lead borate sample than in the samples that were only stage dried. Figure $2.14 \mathrm{~F}$ is a dark field image of the same region in Figure $2.17 \mathrm{C}$ using the diffracted electrons of both PBZT reflections and crystallized lead borate. The small bright regions are diffracting particles now more visible in the hot pressed samples due to the increased image contrast from crystallization.

The particle size distribution can be qualitatively estimated as bimodal with the large particles on the order of $100 \mathrm{~nm}$ and the smaller particles approximately $10 \mathrm{~nm}$ in 
diameter. Thus, the lead borate is not continuous at the 28 to 30 per cent weight loading in either the stage dried or the hot pressed samples.

The original volume of water in as-coagulated PBZT from 5\% solids spinning solution has been shown to be about $85 \%$ [Cohen, 1987]. The maximum volume fraction of glass attainable is limited because the initial infiltrant is a solution of the solgel glass precursor and upon heat treatment the precursor undergoes densification.

Water swollen PBZT as coagulated film was infiltrated with epoxy by the procedure outlined in the experimental section. Measurement of the neat PBZT film width before and after epoxy impregnation indicates no significant shrinkage occurred.

Figure 2.18 compares a cross section of the epoxy impregnated as coagulated biaxially oriented PBZT film with a higher magnification cross section of the heat pressed PBZT/lead borate sample. The microfibrillar network first observed by Cohen in PBZT fibers is evident in the bright field TEM image. The larger lead borate particles in Figure 2.17B are approximately the same size as the voids between the microfibrils in Figure 2.17A.

Figure 2.19 shows bright and dark field images of the PBZT/ borosilicate glass composite film. The images show the same layered structure with fibrils 10 to $20 \mathrm{~nm}$ in diameter as seen in the lead borate soNgel composites. Moreover, the contrast between some layers is high, which may indicate that the borosilicate glass concentrates between the PBZT sheets. The scattering by the borosilicate is less than that observed for the lead borate, so the general image contrast is less. The viscosity and surface tension of the borosilicate infiltrant may cause collapse of the microfibril network because of the osmotic pressure; the sol-gel precursor only penetrates between the layers and not into the microfibrillar structure. No particles are evident in these images as in the lead borate glass sample. 
Figure 2.20 is a bar graph showing the effect of infiltrated glass content and type upon the mechanical properties of the balanced biaxial films [Kovar, 1987]. The different glasses all lower the tensile modulus of the films. The infiltrated glass is also detrimental to the tensile strength properties.

One would not expect a particulate morphology to help to increase the compressive strength of the impregnated film. The mechanism of compressive failure in these polymers has been proposed to be an instability due to the anisotropic elastic constants or the shear strength [De Teresa, 1986, Allen, 1989, Argon, 1972]. These hypotheses suggest that to increase the compressive strength of polymers like PBZT, increases in either the shear modulus or strength must be realized. It has also been proposed that the impregnated glass should be intimately associated with the PBZT fibrils (perhaps in the form of a coating) for the glass to be effective [Kovar, 1987]. The observed borosilicate layers would be expected to give more support than a particulate morphology.

SEM images indicate the silica glass forms layers in infiltrated PBZT film [Kovar et al., 1987]. The $\mathrm{SiO}_{2}$ infiltrated PBZT films show an increase in compressive strength compared with PBZT films when laminated with PEKK as the adhesive [Kovar et al., 1989].

\subsection{Conclusions}

The PBZT orients in the films at a $+/-35-40$ degree angle inclined from the machine direction. The SEM and X-ray data indicate a continuous twist of the PBZT chain axis direction through the $z$ direction of the films. The data indicates the change in orientation direction is gradual near the surface and the change is rapid with respect to distance within a small region in the interior. 
The $\mathrm{I}_{010} / \mathrm{I}_{100}$ intensity ratio depends on the incident $\mathrm{x}$ ray beam direction. The [010] planes are preferentially parallel to the $y-z$ plane, i. e. in the film plane. The observed PBZT layering may therefore have a molecular origin; specifically, the orientation of the aromatic rings is in the plane of the layers.

The sol-gel infiltrate contains a large amount of voids after drying. For the glass composite stage dried $\left(300^{\circ} \mathrm{C}\right)$ samples, the void size observed from the $\mathrm{X}$-ray samples was found to be 0.3 to 1.5 nanometers with the average being 1.2 nanometers. The voids had no preferred orientation. The densification heat treatment causes a loss of the diffuse scatter due to voids in the glass. The void loss is a function of temperature and probably is a function of time and pressure as well.

This work demonstrates the feasibility of infiltration of sol/gel glass into the microfibrillar as-coagulated structure of the PBZT films. The morphology demonstrates that infiltration of the glass is successful. However, the low amount of glass that results from the precursor infiltrant, the final volume fraction of glass impregnant within the PBZT film or fiber. 


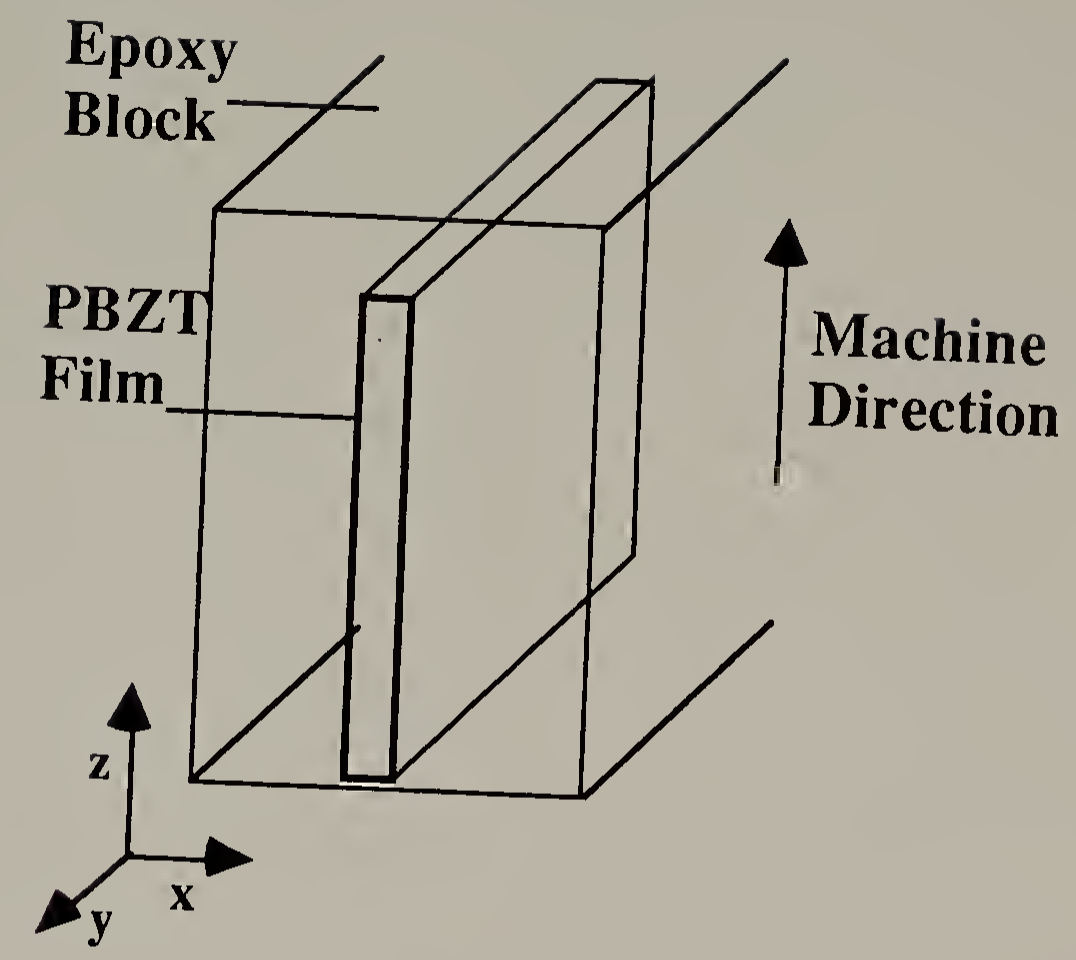

Figure 2.16 The schematic demonstrates the orientation of the film samples for
microtoming. 
틀

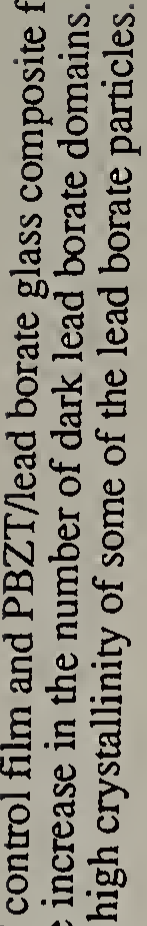

$5 \cong$

¿ $\frac{1}{2}$

फैं 되

ณ월

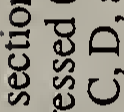

寻芯

ᄂ ญ્

음

路 응

畐

귱

늘

동잉

哥

呦

陆雚

공 


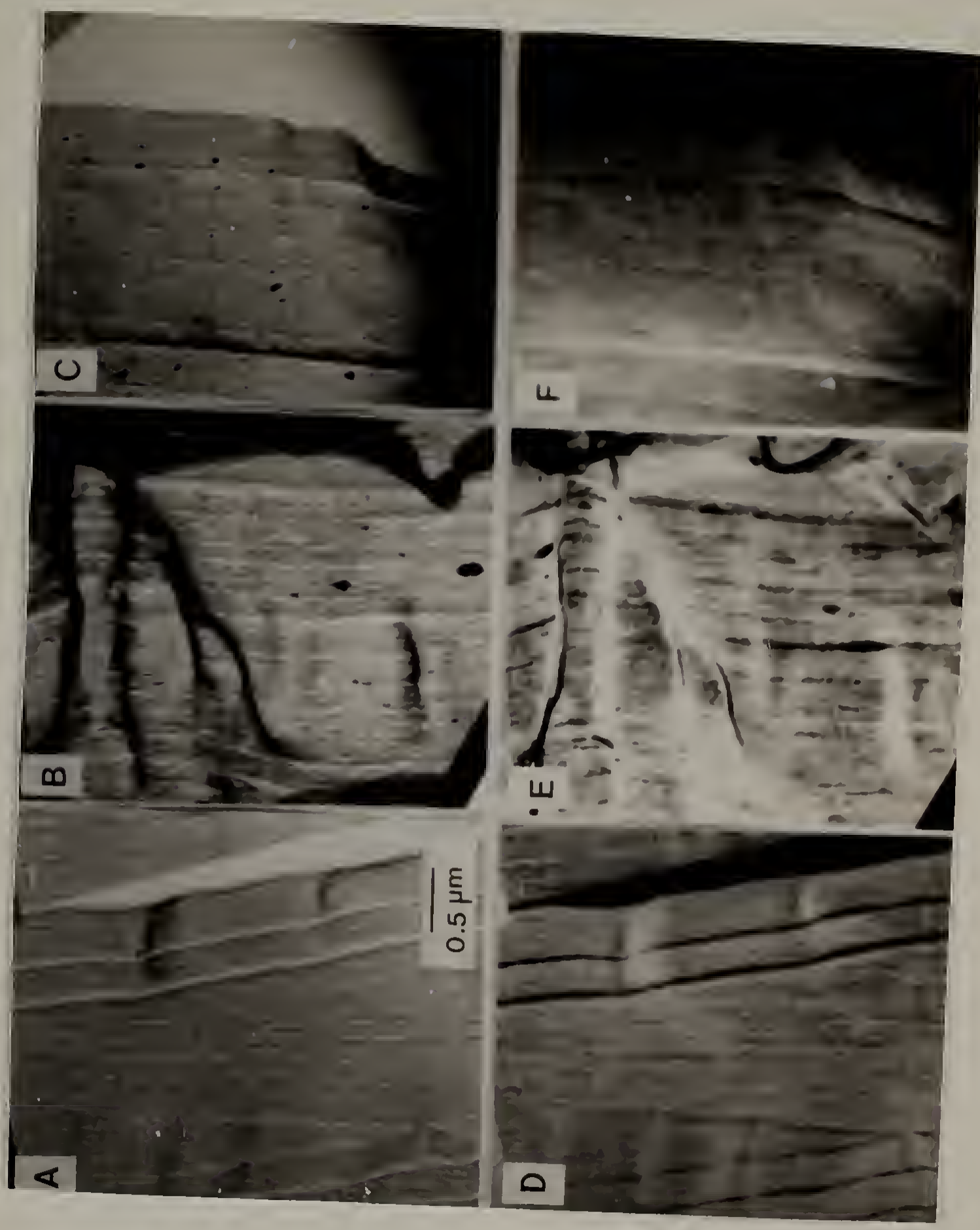




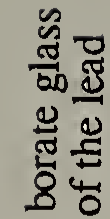

ฐू.

5

m둥

政

o

항

틀

흔

.

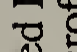

콜

ㄴ.

ह्ञ

ฟ.

영눙

䨌?

ตั

융

ن

क क

宅

प्to

\&े.

용

承

을

运品

要递

结莒苋

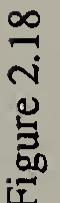




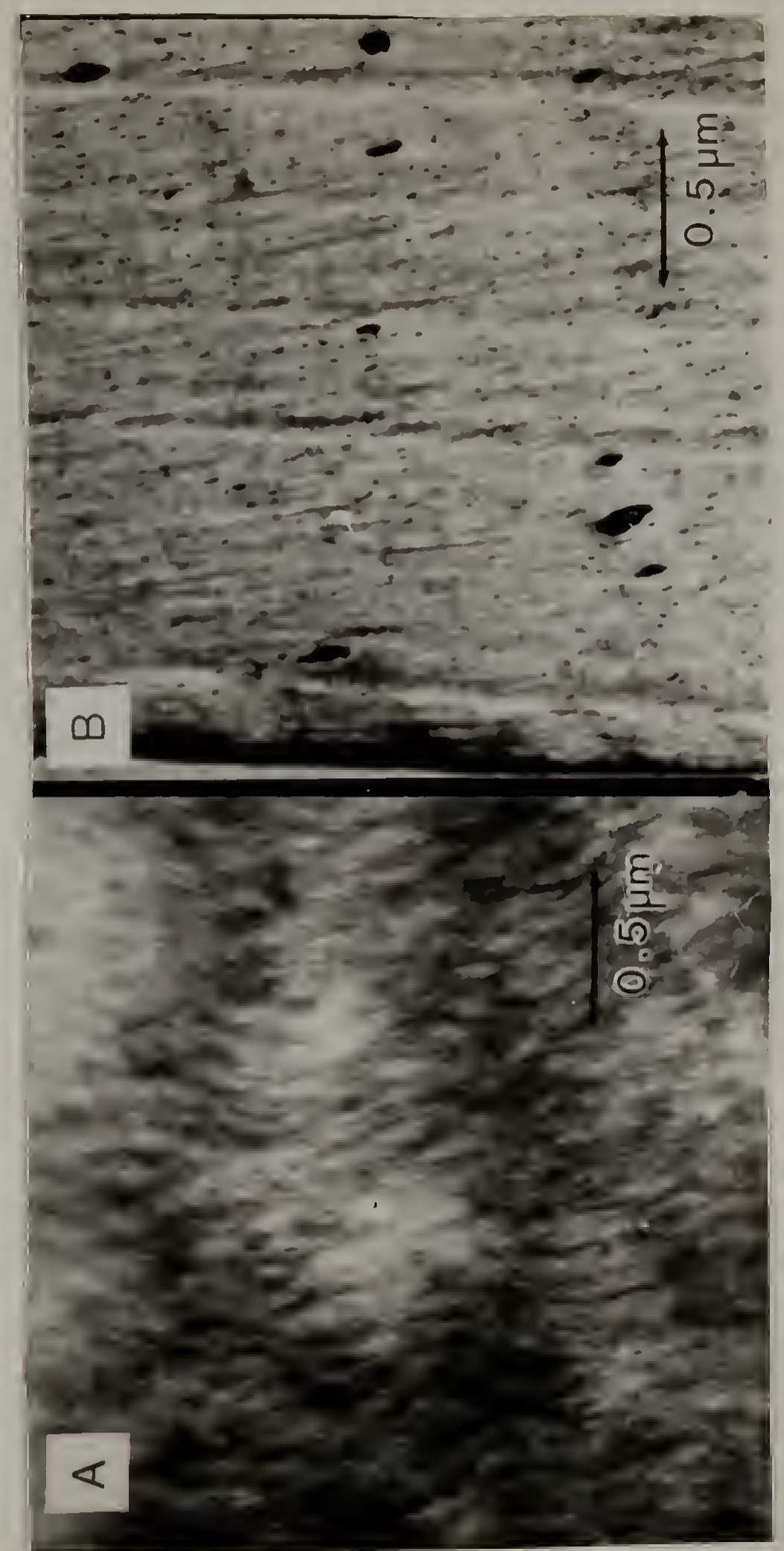




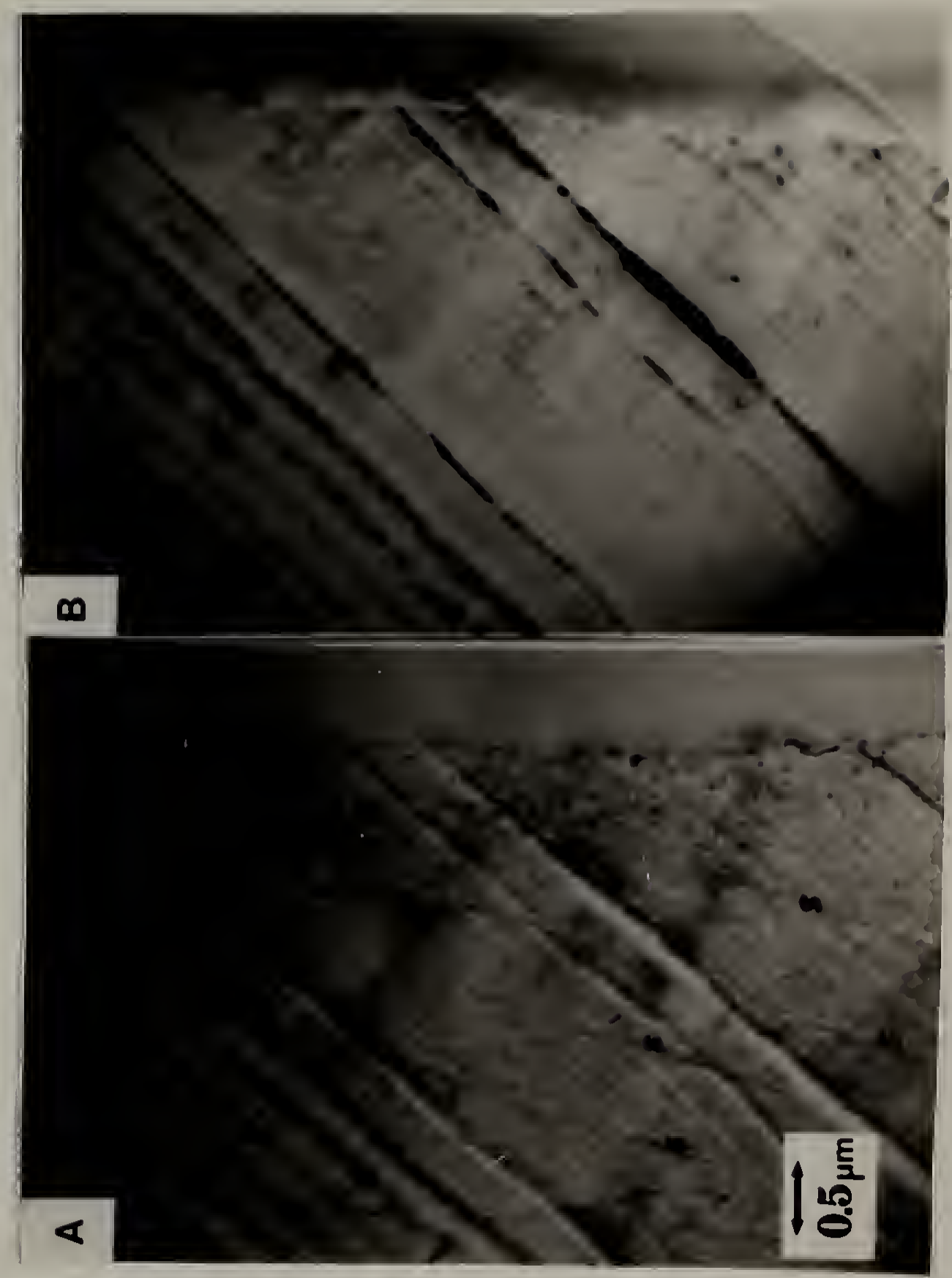




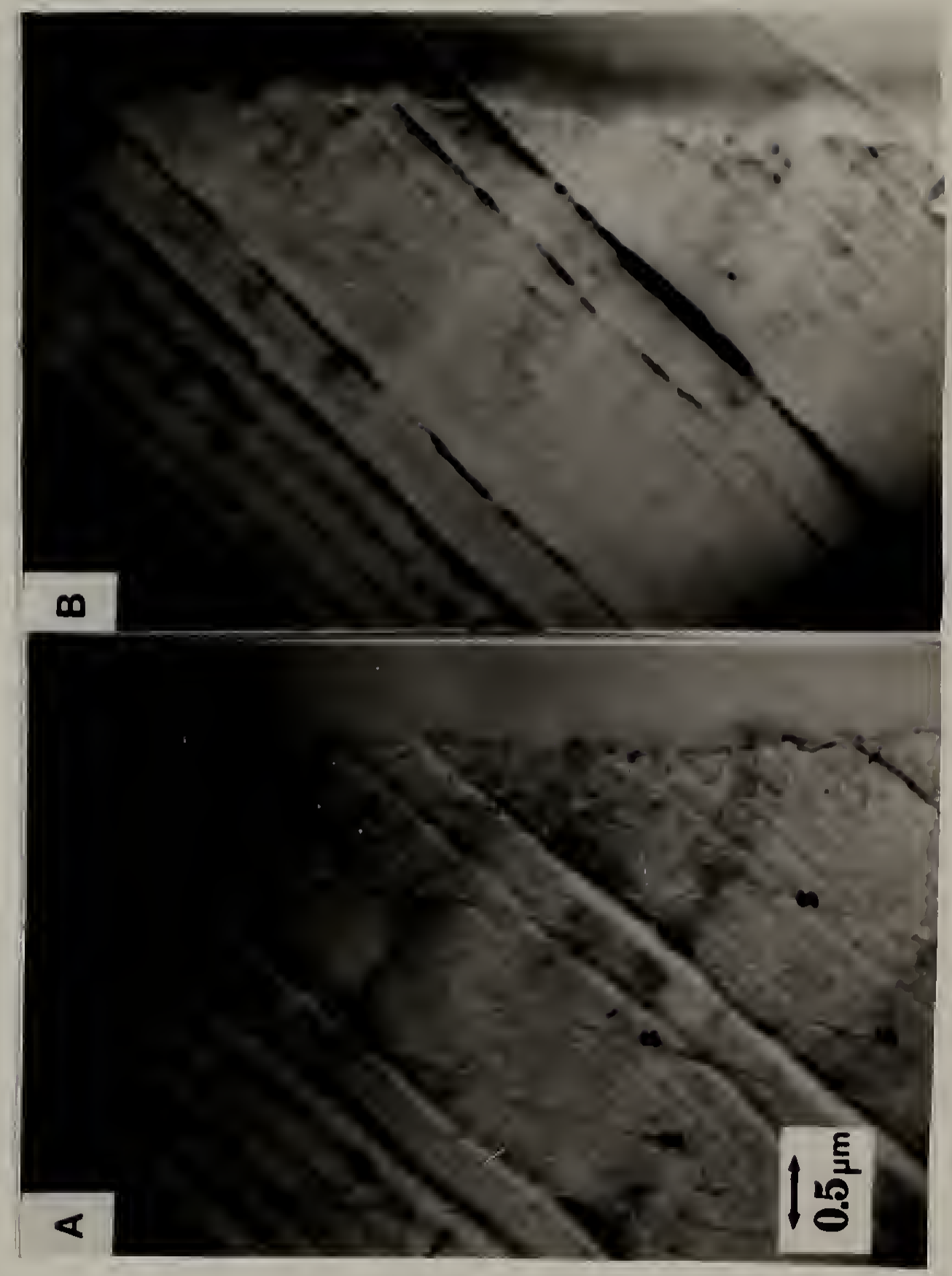



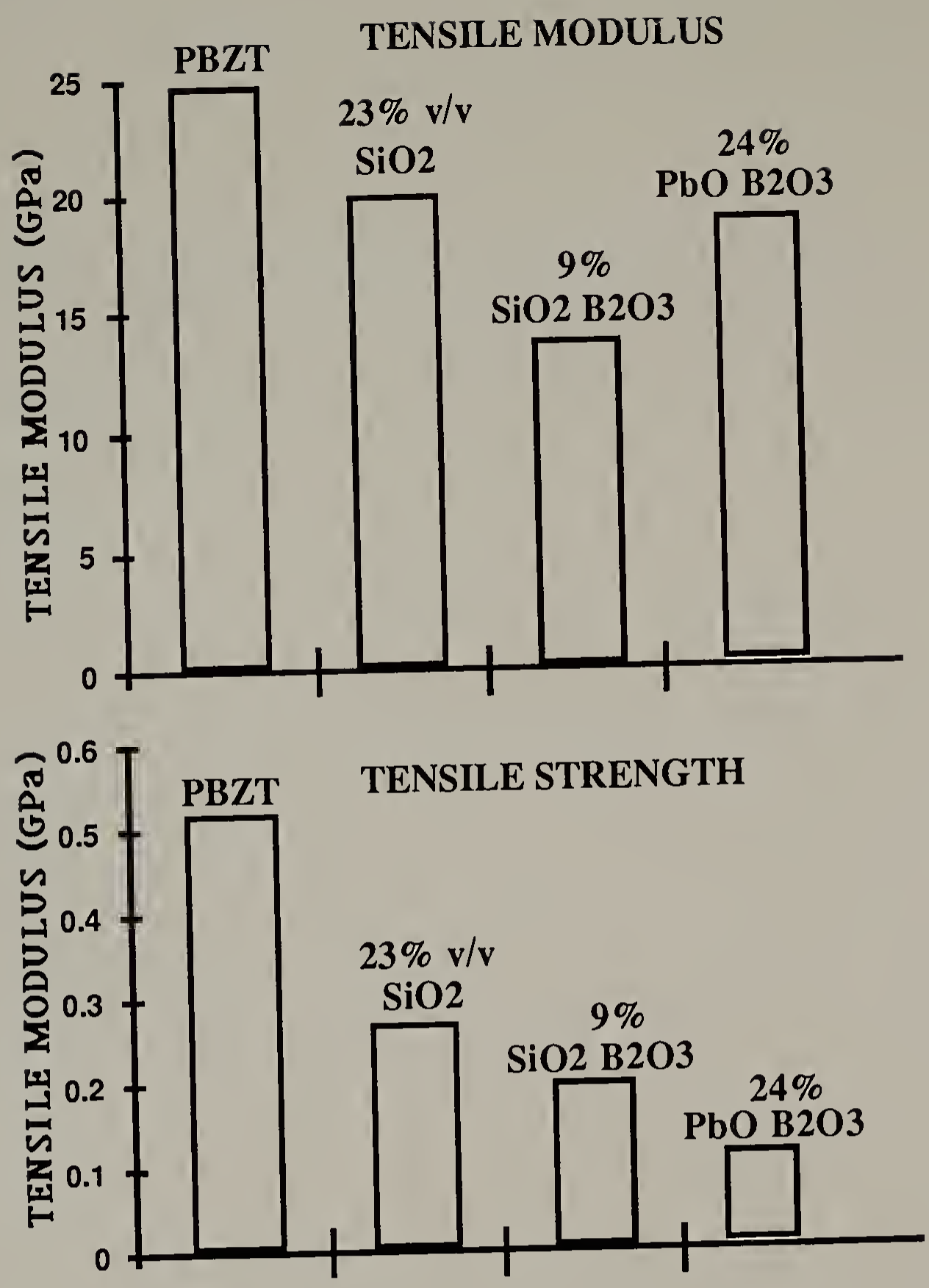

Figure 2.20 Bar graphs of tensile properties for biaxially balanced films with various types of glasses and glass contents. 


\section{$2.5 \quad$ References}

Alexander, L. E., X-Ray Diffraction Methods in Polymer Science, Robert E. Krieger Publishing Co., Malabar, FL, 1985.

Allen, S. R., Ph. D. Thesis, University of Massachusetts, Amherst, MA (1983).

"Mechanical and Morphological Correlations in Poly(p-phenylene benzobisthiazole) Fibers".

Allen, S. R., Journal of Materials Science, 22 , pp. 853-859(1987). "Tensile recoil measurement of compressive strength for polymeric high performance".

Allen, S. R., Polymer, 29, pp. 1091-1094(1989). "Stress-coupling phenomena in anisotropic fibres".

Argon, A. S., Treatise on Materials Sciences and Technology, Volume 1, Academic Press, New York, (1972). "Fracture of Composites".

Cohen, Y., "Structure Formation in Solutions of Rigid Polymers Undergoing Phase Transitions", AFWAL-TR-87-4030, AFWAL/MLBP, Wright Patterson Airforce Base, April, 1987.

Cohen, Y., Farris, R. J., and De Teresa, S. J., U.S. Patent Number 899910, 25 August 1986. "Novel Composites based on Reinforcement with Microfibrillar Networks of Rigid-Rod Polymers".

Cohen, Y., Farris, R. J., and De Teresa, S. J., U.S. Patent Number 4842924, 27 June 1989. "Novel Composites based on Reinforcement with Microfibrillar Networks of Rigid-Rod Polymers".

Cohen, Y., Farris, R. J., and De Teresa, S. J., United Kingdom Patent 2195672, 14 August 1990. "Novel Composites based on Reinforcement with Microfibrillar Networks of Rigid-Rod Polymers".

Cohen, Y., Farris, R. J., and De Teresa, S. J., U.S. Patent Number 5102601, 7 April 1992. "Process for Fabricating Novel Composites Based on Reinforcement with Microfibrillar Networks of Rigid-Rod Polymers".

DeTeresa S. J., Ph. D. Thesis, University of Massachusetts, Amherst (1986). "Axial Compressive Strength of High Performance Polymer Fibers".

Fieser, R. G. and Goldsmith, C. C., Glass Technology, 19, pp. 10(1978). "Devitrification studies of Corning 7575 seal glass".

Hermans, J. J., Hermans, P. H., Vermaas, D., and Weidinger, A., Rec. trav chim. Paysbas, 65 , pp. 427 427(1946). "Quantitative Evaluation of Orientation in Cellulose Fibres from X-ray Fibre Diagram". 
Hindeleh, A. M. and Johnson, D. J., Polymer, 1980, Volume 21, page 929. "An Empirical Estimation of Scherrer Parameters for the Evaluation of True Crystallite Size in Fibrous Polymers".

Kovar, R. F., AFB-0022.FM-8689-3, Airforce Office of Scientific Research, August, 1987. "Microcomposite Processing and Applications".

Kovar, R. F.; Haghighat, R.; and Lusignea, R. W.; Materials Research Society Symposium Proceedings, Vol. 134, (1989). "Improvement of Compressive Strength in Ordered Polymer Films and Fibers by Sol-Gel Processing".

Kovar, R. F. and Lusignea, R. W., AFB 0097-FM-8535-41, Air Force Office of Scientific Research, March, 1986. "Microcomposite Processing and Applications".

Krough-Moe, J. and Wold-Hansen, P. S., Acta Cryst., B29, pp. 2242-2246 (1973). "The Crystal Structure of Hexalead Pentaborate, $6 \mathrm{PbO} .5 \mathrm{~B}_{2} \mathrm{O}_{3}$ ".

Lusignea, Richard W., Materials Research Society Symposium Proceedings, Vol. 134, (1989).

McMurdie, Howard F. and Elmer N. Bunting, Journal of the National Bureau of Standards, 23, page 543 (1939). "X-ray Studies of Compounds in the System $\mathrm{PbO}-\mathrm{SiO}_{2}$.

Minter,J. R., Structural Investigation of Fibers and Films of Poly(pphenylenebenzobisthiazole), AFWAL-TR-82.4097, Vol I, Materials Laboratory (AFWAK/MLBP), May 1982.

Newman, K. E., Zhang, P., Cuddy, L. J., and, Allara, D. L.; Journal of Materials Research 6 , pp. 1580-1594 (1991).

Perloff, A., and Block, S., Acta Cryst., 20, pp. 274 (1966). "The Crystal Structure of the Strontium and Lead Tetraborates, $\mathrm{S} r \mathrm{O} .2 \mathrm{~B}_{2} \mathrm{O}_{5}$ and $\mathrm{PbO} .2 \mathrm{~B}_{2} \mathrm{O}_{3} "$.

Pottick, L. A., Ph.D. Thesis, University of Massachusetts, Amherst, MA (1986). "The Influence of Drying on the Structure and Mechanics of Poly(p-Phenylene Benzobisthiazole) Fibers". 


\section{CHAPTER 3}

\section{LIQUID CRYSTAL POLYMER FIBER BLENDS BY SOLUTION BLENDING}

\subsection{Introduction}

Most investigators study blends of lyotropic liquid crystalline polymer and coil type linear polymer by spinning fibers from relatively low concentration isotropic solutions attempting to attain composites of rigid molecules dispersed on a molecular level in a coil polymer matrix [Kumar et al., 1988; Hwang et al., 1983, Takaynagi, 1984].

The formation of a microfibrillar network in these systems was first evident from mechanical data of as-spun wet fibers [Pottick, 1986]. Morphological evidence of the microfibrils was presented by replacement of the water with epoxy resin and subsequent electron microscopy and small angle X-ray scattering data [Cohen and Thomas, 1988]. Figure $2.19 \mathrm{~A}$ in the previous chapter shows TEM micrographs of such an infiltrated film.

Poly(para-phenylene benzobisthiazole) or PBZT has also shown to form a microfibrillar network upon coagulation from isotropic solution blends with flexible coil polymers [Hwang, 1989]. Such work questions the feasibility of molecularly disperse blends of rigid rod polymers such as PBZT. The diffusion controlled process of coagulation and the relative solubilities of the blend components determines the morphology of the final blend [Hwang, et al., 1991a]. For example, the network formed during coagulation from an isotropic nylon/PBZT solution has the same tensile and compressive properties as a nylon infiltrated PBZT fibrillar network with the same materials [Hwang, et al., 1991b]. 


\subsubsection{Liquid crystal solution blends}

The advantages of spinning an immiscible solution blend of liquid crystal polymers and coil polymers become evident if one considers several factors. First, higher concentration solutions are wanted for fiber spinning for practical reasons. Second, experimental evidence indicates that phase separation will occur even at low concentrations [Hwang, 1989]. Third, at higher concentrations the liquid crystalline polymer will have anisotropic phases that exclude any coil polymer for thermodynamic reasons. Solutions of lyotropic liquid crystal polymers in the anisotropic region of the phase diagram will not form single phase solutions with the addition of coil polymers [Flory, 1978].

Investigations of fibers spun at solution concentrations such that the solution is in the single phase liquid crystal region or the biphasic region of the phase diagram reveal that the resulting fibers contain two phases the rigid rod polymer as a minority component in the blend [Krause et al., 1986 and 1988]. Spinning from multi-domain mixtures may offer further advantages in subsequent processing (such as eliminating preimpregnation before lay-up) to obtain final structural components. The use of higher melting liquid crystal polymers in matrices with lower melting points is cited as a method of forming composite structures consisting of liquid crystal polymer reinforcing fibers formed in-situ in a thermoplastic matrix [Bassett and Yee, 1990]. The investigation of how spinning processing variables affect the final morphology on various length scales may indicate which process parameters are dominant, and give direction on which processing condition would yield novel structures.

\subsubsection{Rheological behavior of multi-phase systems}

Magliochetti [1989] performed steady shear rheological experiments on a select pair of quaternary solutions, which are isotropic and biphasic mixtures of PBZT, coil 
polymer, poly(phosphoric) acid (PPA), and methane sulfonic acid (MSA). He found support for proposed shear-induced orientation hypothesis for biphasic solutions. Explicitly, the application of a steady shear causes a decrease in the dynamic moduli, linear relaxation modulus and discrete relaxation spectrum due an increase in molecular orientation [Magliochetti, 1989]. There are no characterizations of the effects of elongational flows found in the fiber spinning process of such blends.

This chapter summarizes morphological characterization of spinning dopes, fibers, and composite structures made from poly(phenylene benzobisthiazole) (PBZT)/poly(ether ketone ketone) (PEKK) and PBZT/aromatic amorphous polyamide blends.

\subsection{Experimental}

\subsubsection{Materials}

E. I. du Pont de Nemours and Company Inc. supplied the PBZT/PEKK and PBZT/Zytel aromatic polyamide fiber, composites, and spinning dope samples and solutions for this study. Figure 3.1 shows the general chemical structures and solvents. Magliochetti examined these spinning dopes in steady shear rheological experiments [Magliochetti, 1990].

\subsubsection{Mixing}

For dope morphology experiments, two solutions were mixed; 50.0 grams of a $11.0 \%$ solids PBZT/PPA solution (from E. I. du Pont Co.) and 47.0 grams of a 5.2\% solids Zytel 330/MSA solution, yielded a 69/31 PBZT/Zytel 330 (11\% solids) spinning dope with a 50/50 PPA/ MSA solvent mixture.

The Atlantic mixer held the PBZT spinning dope under a nitrogen gas purge while mixing with the speed set at 5 and the mixing chamber's heating oil set at $45^{\circ} \mathrm{C}$. A 
thermal couple on the exterior of the mixing assembly indicated the chamber temperature remained within the range of $40-44^{\circ} \mathrm{C}$ throughout the experiment. The mixer ran at full speed after addition of the polyamide solution to mixing chamber. Removing samples from the mixer at various time intervals permitted examination of the effect of mixing time on dope morphology. Nitrogen purged glass vials (stored in a freezer to prevent reorganization) held the mixed spinning dope samples until microscopic observation.

A slight shearing of a small quantity spinning dope between two glass slides allows observation the structure of the spinning dope by forming a preferentially oriented thin film of PBZT. A nitrogen gas purged glove bag kept moisture from contacting the spinning dope during sample preparation. Placing the slides into a small Ziploc bag excluded moisture. Cutting and taping the edges of the polyethylene bag to the upper glass slide permitted a window free of polyethylene for viewing of the sheared spin dope between cross polars on a Zeiss polarizing optical microscope. Polaroid Type 55 P/N black and white film was used to record the images.

\subsubsection{Fiber spinning}

Figure 3.2 is a schematic of a spinning apparatus used for fabricating fibers. The spinning apparatus could successfully produce fibers with the spinning dope at a temperature of $60^{\circ} \mathrm{C}$ and the air pressures of 300 to $400 \mathrm{psi}$. The spinning dope extruded directly into a bath containing $50 / 50 \mathrm{MSA} / \mathrm{H}_{2} \mathrm{O}$ maintaining a one half inch air gap. Using water as the coagulant, similar experiments showed how spin-draw ratio (by varying the take up velocity during spinning) affects the fiber morphology.

A falling stream was used as illustrated in Figure 3.2 when the coagulant was water. The elongational deformation is localized in the region between the die and the top of the falling stream of coagulant. 


\subsubsection{WAXS procedure}

The fibers were mounted on cardboard holders with fiber axis vertical. An evacuated flat film Statton camera recorded WAXS diffraction patterns using a nickel filtered $\mathrm{CuK} \alpha$ radiation (wavelength $=1.544$ Angstroms).

\section{PBZT}

Poly (para-phenylene Benzobisthiazole)

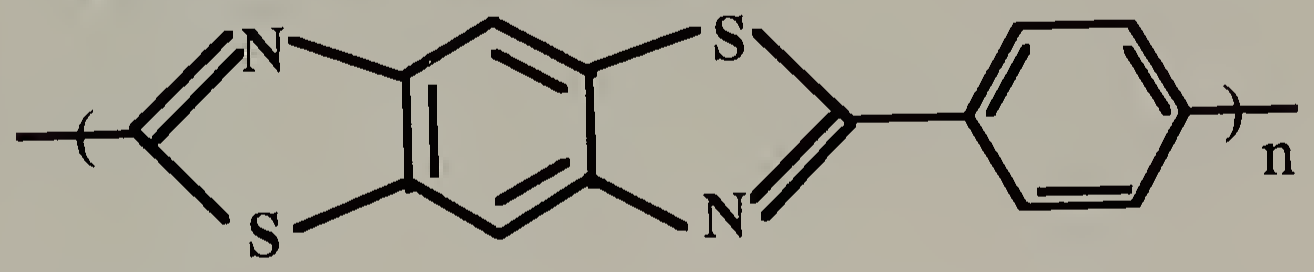

\section{PEKK}

Poly (ether ketone ketone)

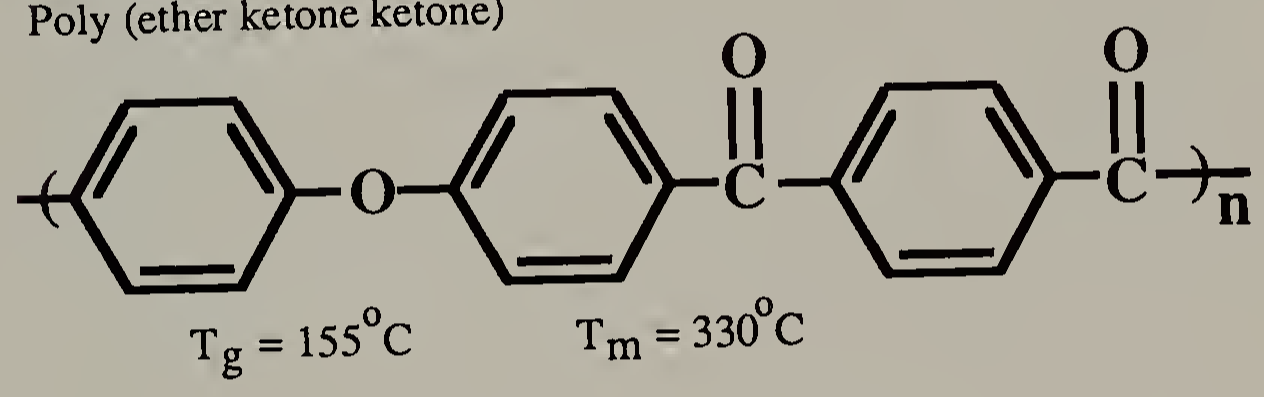

\section{Zytel 330}

Amorphous Aromatic Polyamide

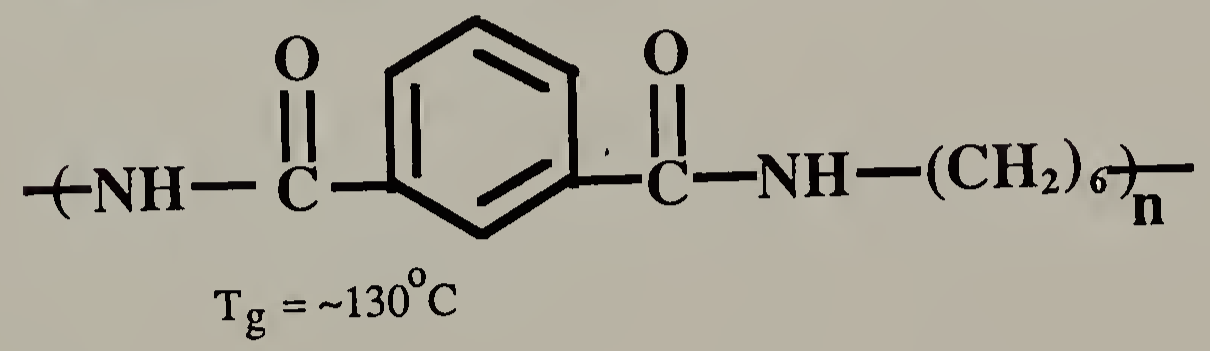

Figure 3.1 The materials used in this study. 


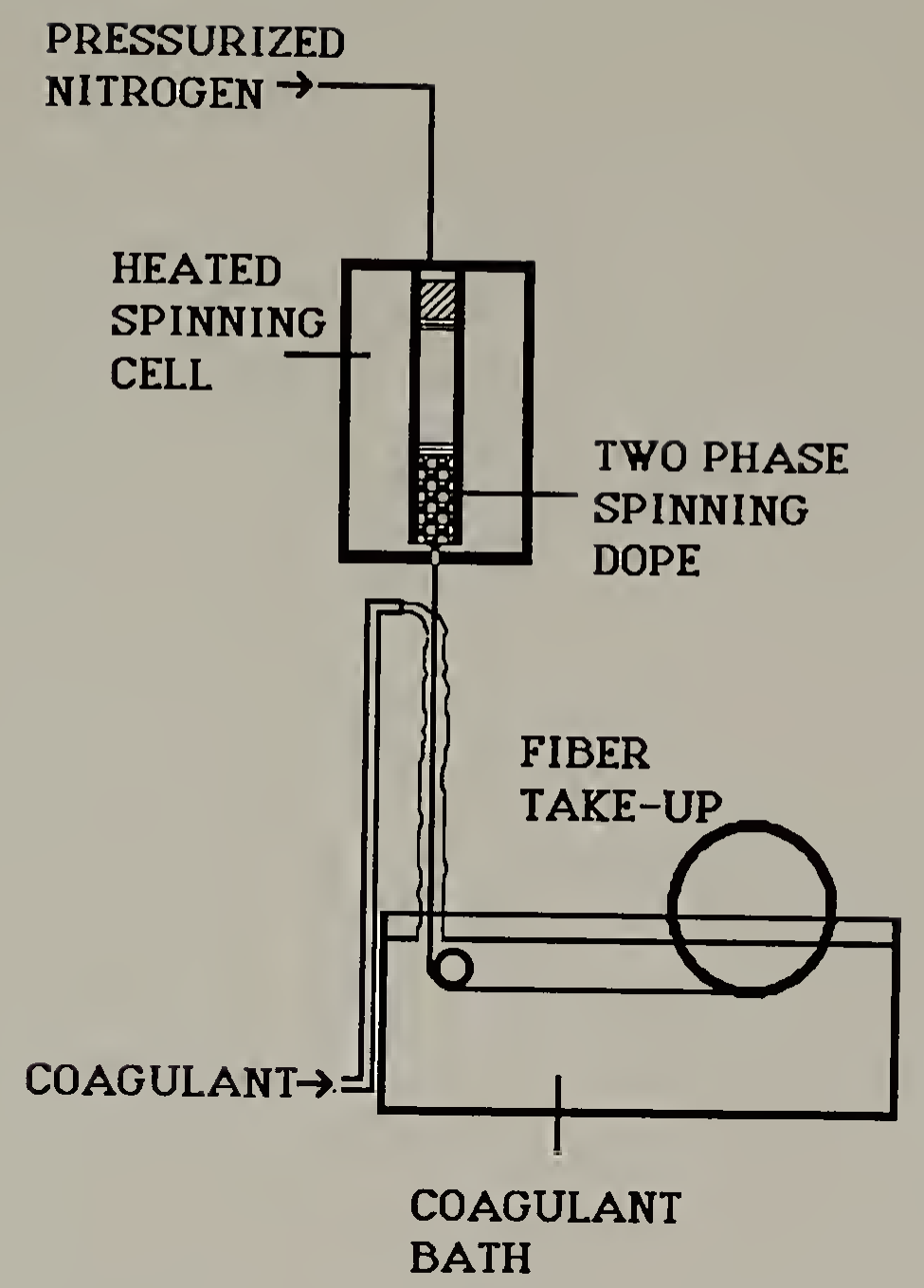

Figure 3.2 A schematic of a fiber spinning apparatus.

\subsubsection{TEM}

Mounting the fiber and composite specimens in a silicon rubber or polyethylene molds facilitates microtomy for cross and longitudinal sections. Pelco Medcast QuikMix Kit epoxy of medium hardness was the embedding medium for the fiber and composite specimens. The epoxy cured at $60^{\circ} \mathrm{C}$ for 16 to 20 hours.

Embedding the samples in epoxy blocks allowed trimming of the specimen to 1 square millimeter permitting microtoming on a Sorvall MT2-B ultra-microtome with a diamond knife at ambient temperatures. Floating the sections on water permitted retrieval 
on copper grids for TEM examination. The sections were 150 to 300 nanometers thick as determined by interference colors observed as the specimens were floating on water.

Orientations for the views of the micrographs concerning the fiber axis are given in Figure 3.3. Kodak SO-163 electron image film was used to record the TEM images of the film thin sections on a JEOL 2000FX TEM at $200 \mathrm{kV}$. The microtomed epoxy block faces were also examined with a Zeiss optical microscope using 546 um wavelength light in reflective mode.

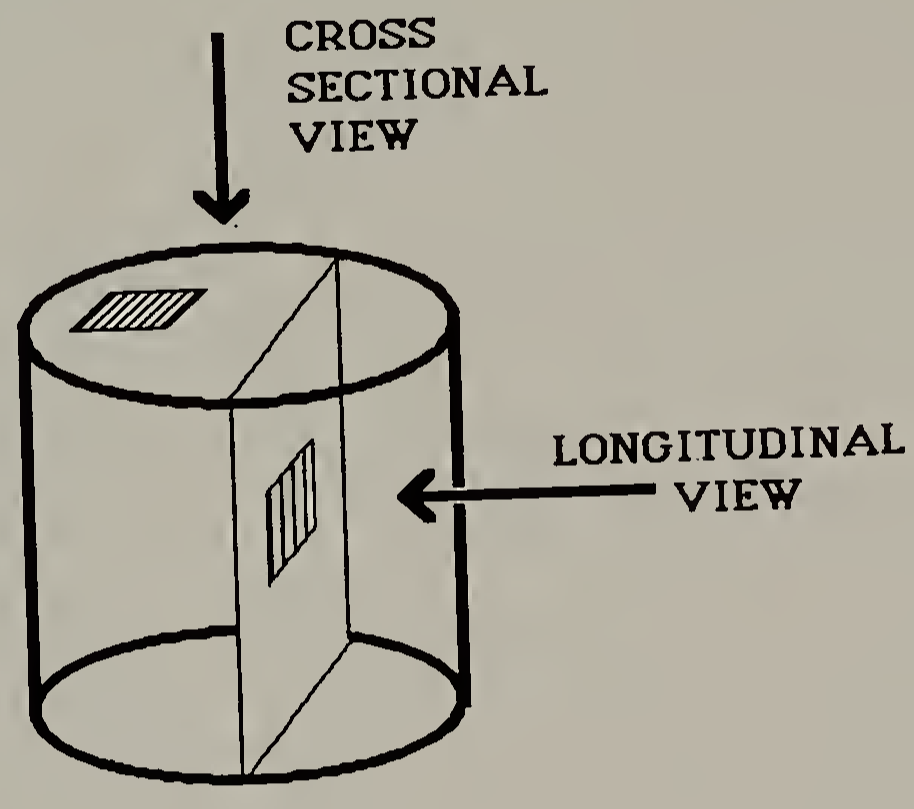

Figure 3.3 Orientational of views for TEM images.

3.3 Results and Discussion

\subsubsection{Mixing of spinning dopes}

This section discusses the amount of mixing needed to obtain a fine dispersion of the coil polymer solution in the nematic PBZT solution, because poor dispersion should greatly influence the properties of the fibers. Figure 3.4 compares the structure observed in a polarizing microscope of a PBZT/Zytel 3369FX spinning dope and a neat 
PBZT spinning dope both containing $11 \%$ solids. When sheared between glass microscope slides, the PBZT molecules in both solutions orient in one principal direction.

The image in Figure 3.4A is the PBZT/Zytel 3369FX solution mixture taken without any polarizers and Figure 3.4B is the same sample area with the polarizers crossed to extinction at an angular orientation such that the transmitted light is at maximum intensity. The droplets in the mixture remain extinct when rotating sample between the cross polars; the extinction indicates these areas are isotropic and are probably the polyamide solution droplets.

The refractive index difference between polyamide and PBZT solutions is large enough to easily observe the interface between the phases. Images for the neat PBZT spinning dope in Figures 3.4C and 3.4D show none of the internal structure evident in the PBZT/polyamide spinning dope.

In Figure 3.4A shows the first sample of the PBZT and polyamide solution which appeared macroscopically homogeneous (after 70 minutes of mixing time); one can see that the average domain size is on the order 10 microns.

Figure 3.5 shows a sequence of photomicrographs from the same series of samples. At 2.5 and 3 hours of mixing, the polyamide solution domains are still up to 10 microns in dimensions. At 4.7 hours the dimensions of the domains are comparable to those of a sample taken after mixing for 17.5 hours. The Atlantic mixer is capable of dispersing the minority phase to an average equilibrium domain size of 2 microns. 

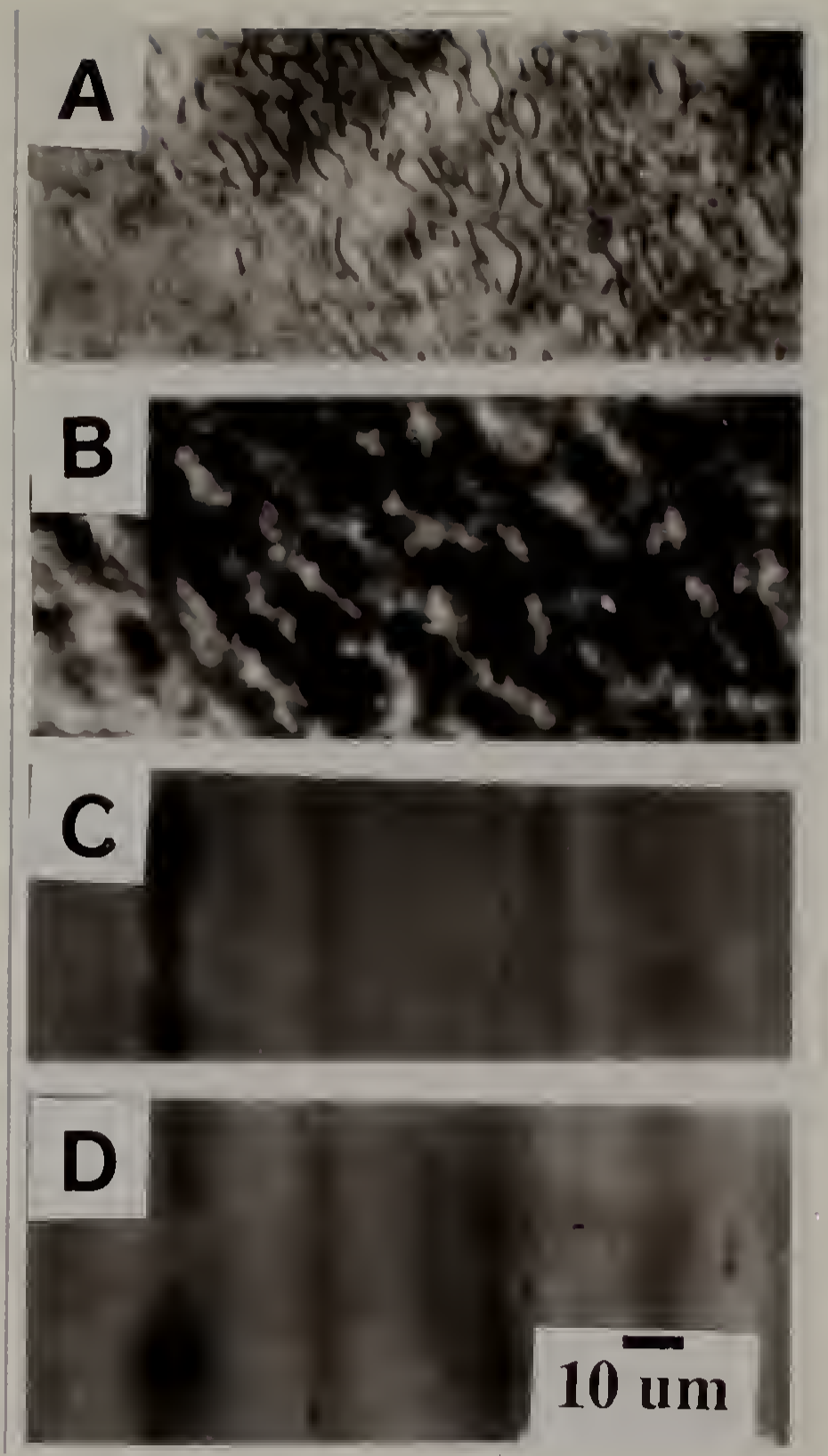

Figure 3.4 A 69/31 PBZT/polyamide (13\% solids) spinning mixture slightly sheared between glass slides in the indicated direction without $(\mathrm{A})$ and with (B) cross polarizers parallel to the image edges. (C) and (D) are images (with and without cross polarizers, respectively, of neat PBZT spinning dope sheared between glass slides that show no domain structure). 


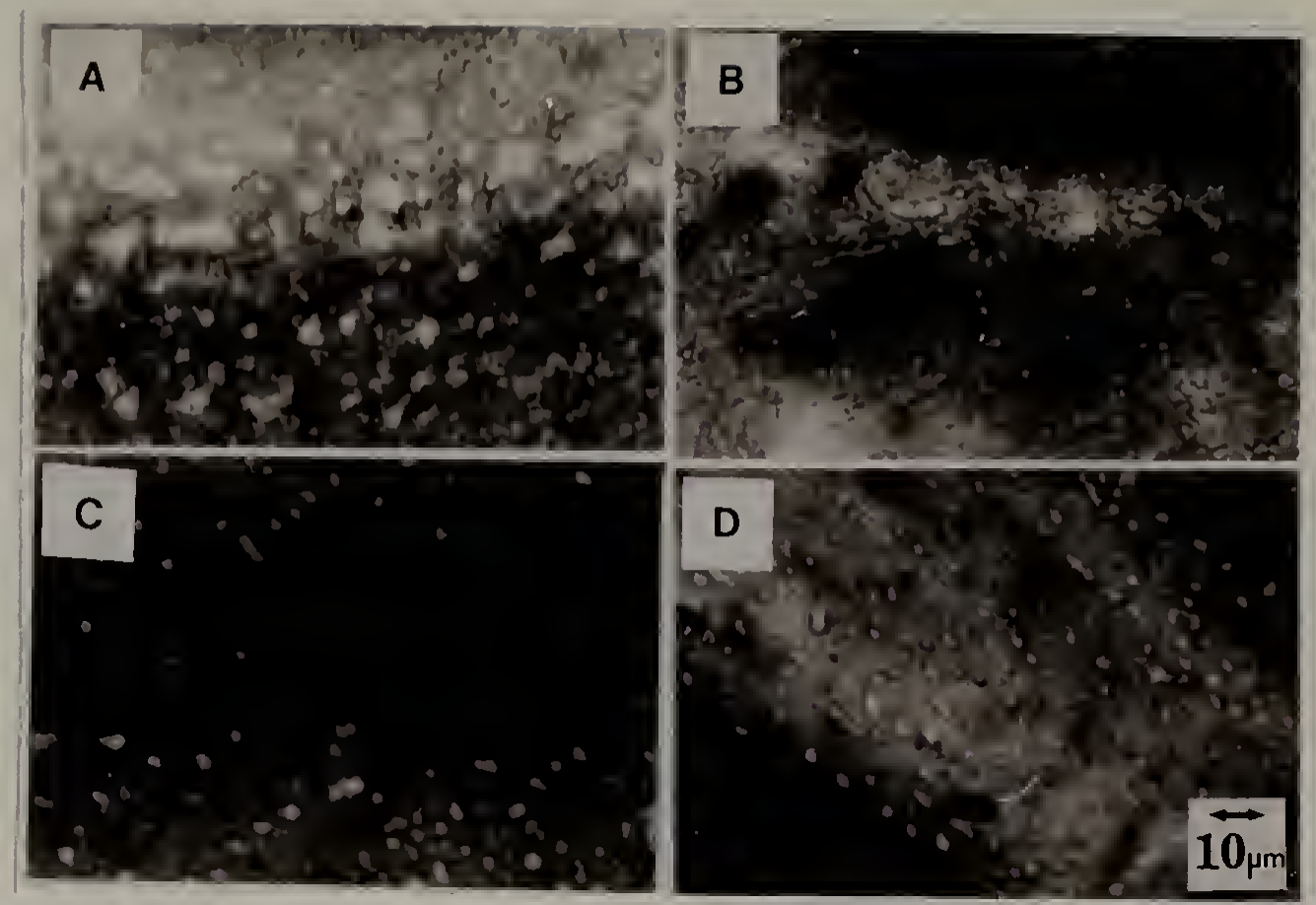

Figure 3.5 Optical micrographs of 69/31 PBZT/polyamide (11\% solids) spinning dope samples between glass slides after mixing for (A) 2.5 hours, (B) 3 hours, (C) 4.7 hours, (D) 17.5 hours The sizes of the polyamide rich regions appear to attain an average steady state size of approximately 2 microns. 
Sample

Designation

Composition

Comments

AS-10

As spun fiber.

60/40 PBZT/PEKK

HT-10

Heat treated fiber $\left(500^{\circ} \mathrm{C}\right)$

60/40 PBZT/PEKK

TA-10

Tape from AS-10

60/40 PBZT/PEKK

MB-10

Bar molded from TA-10

60/40 PBZT/PEKK

HT-PA

Heat treated MC yarn

69/31 PBZT/PA*

AT-PA

Acid treated HT-PA

69/31 PBZT/PA

TA-PA

Tape from HT-PA

69/31 PBZT/PA

MB-PA

Bar molded from TA-PA

69/31 PBZT/PA

PA* FX3369 - an experimental aromatic polyamide similar to Zytel 330

Samples supplied by E. I. du Pont de Nemours and Company Inc.

\subsubsection{Spun PBZT/coil polymer fibers}

Fibers were fabricated from the spinning dope in Figure 3.4 to observe the effect of spin-draw ratio and coagulants on the fiber morphology. When fiber spinning with a $50 / 50$ mixture of methane sulfonic acid and water as a coagulant, fibers could not be spun, except at very low draw ratios.

The microtomed sectioning block of mounted fibers allows optical microscopy examination of the sectioned fibers. Figure 3.6 shows reflective optical micrographs of a microtomed face of epoxy embedded fibers that were coagulated in 50/50 MSA/H2O. The fibers exhibit domains very similar to the structure of the spin dope of Figure 3.4. At the low draw ratio values, the fibers show clearly the elongated domains of coil polymer with a continuous structure of PBZT evident as Figure 3.6b shows. It is 
uncertain whether the polyamide domains are similarly interconnecting or if higher draw ratio values would increase the probability of formation of continuous structures.

TEM bright and dark field images of cross sections of the fiber in Figure 3.7 indicate that as-spun fibers contain voids, since areas with the same electron density as epoxy were evident in the interior of the fiber. Tears and folds in the cross section that are apparent in the image. Cross sections of rigid rod polymers are difficult to obtain without folding or tearing of the specimen. One can discern the underlying morphology in spite of these artifacts of the sectioning process. The PBZT areas can be identified by texture. However, the PBZT was of low crystallinity in the as spun fiber such that the polyamide diffusely scattered with the same intensity as the PBZT, yielding low contrast in the dark field images.

Figure 3.8 shows longitudinal sections of the same fiber in Figure 3.7. The fibers exhibit domains very similar to the structure of the spin dope in Figure 3.4. At this low draw ratio value, the fiber shows clearly the elongated domains of the coil polymer with a continuous structure of PBZT evident.

Figure 3.9 shows reflective mode optical micrographs of fibers spun with water coagulant at draw ratios of 1 and 4, respectively. The water coagulated fiber shows no obvious difference in morphology from the acid coagulated fiber. The optical micrographs in Figure 3.9 indicate fiber cross sections are not circular in shape. The irregular shapes suggest that the fiber is deformable in cross section during the coagulation process while the coil polymer is solvent swollen. The importance of the non-circular cross sections will be discussed in a subsequent section. 

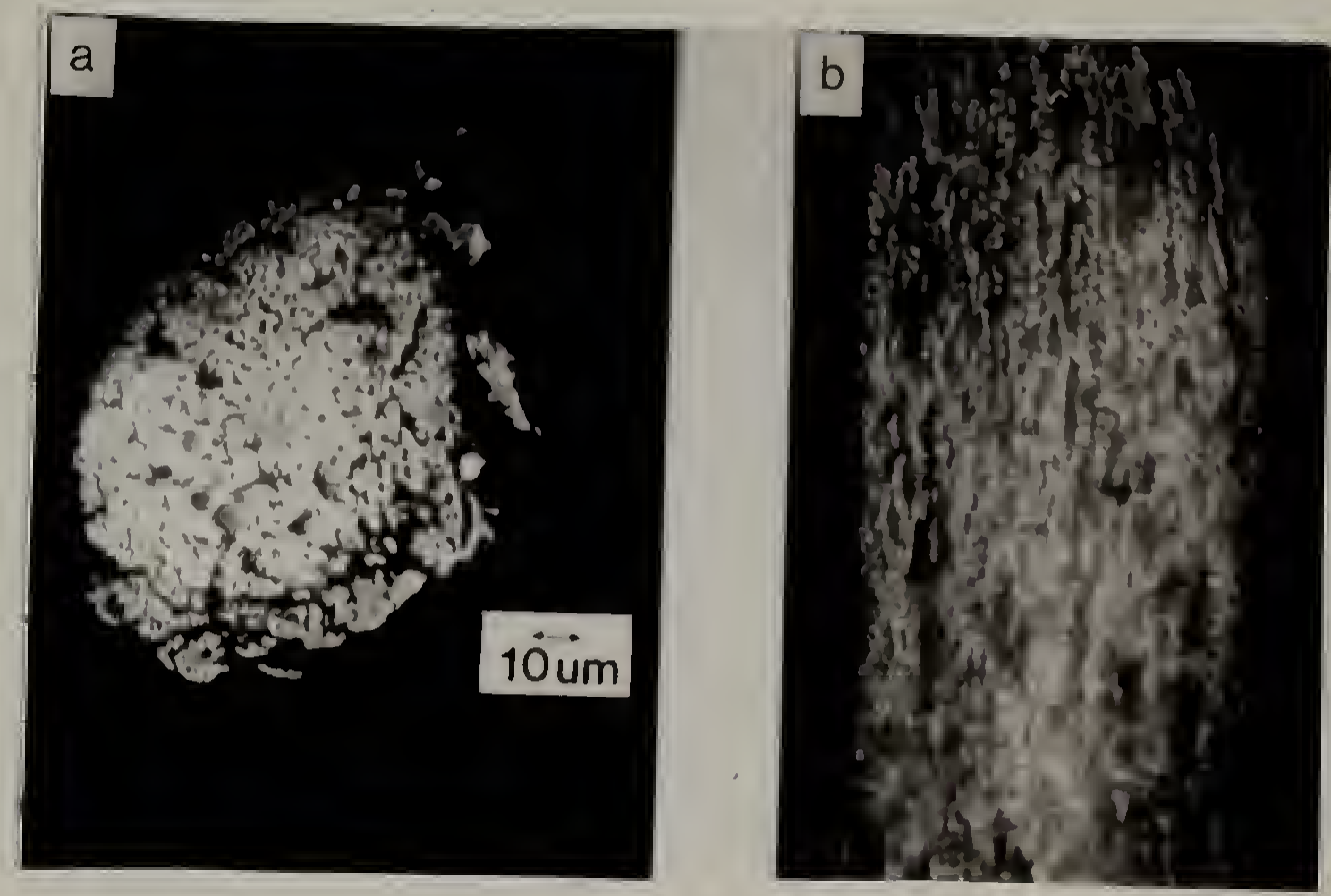

Figure 3.6 Reflective optical micrographs of sectioning block showing (A) a cross section and (B) a longitudinal section of a fiber composed of 69/31 PBZT/polyamide fiber spun at spin stretch ratio of 1 . 
A

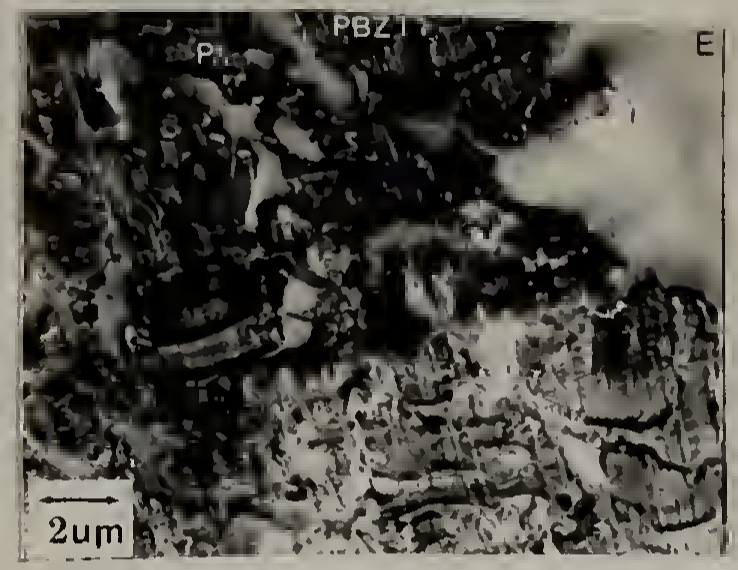

B

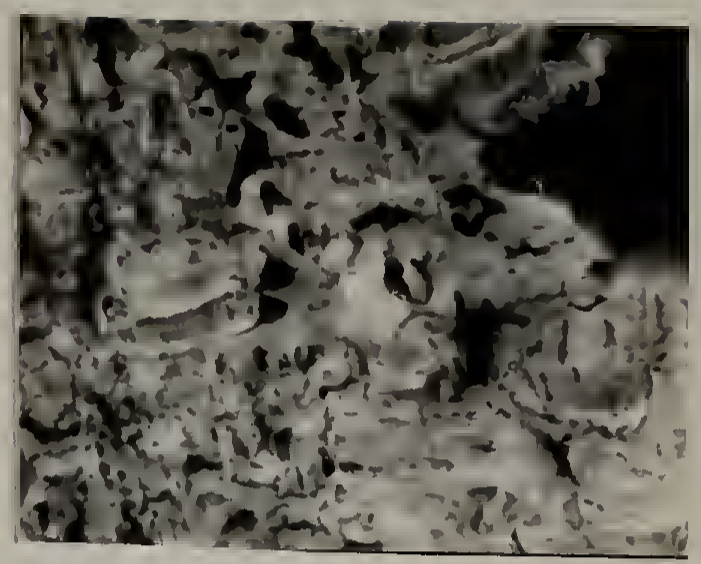

Figure 3.7 TEM images showing (A) bright and (B) dark field images of cross section of a fiber composed of 69/31 PBZT/polyamide fiber spun at spin stretch ratio of 1 .

A

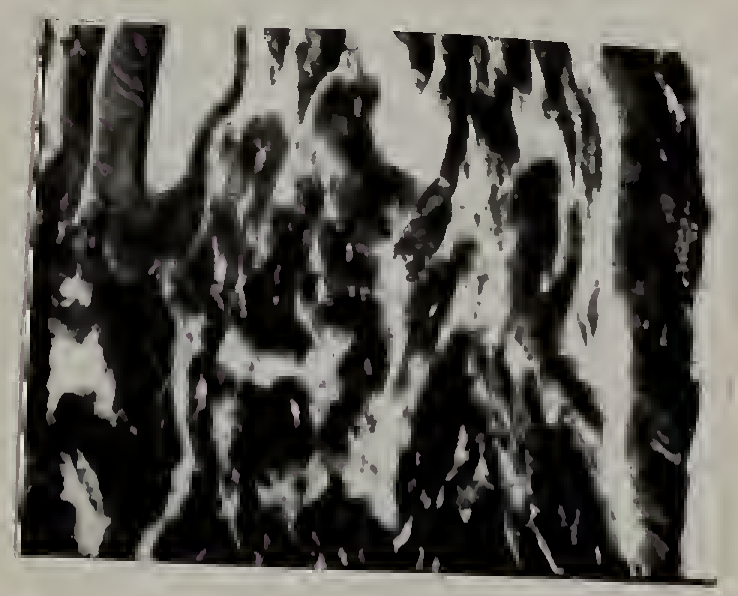

B

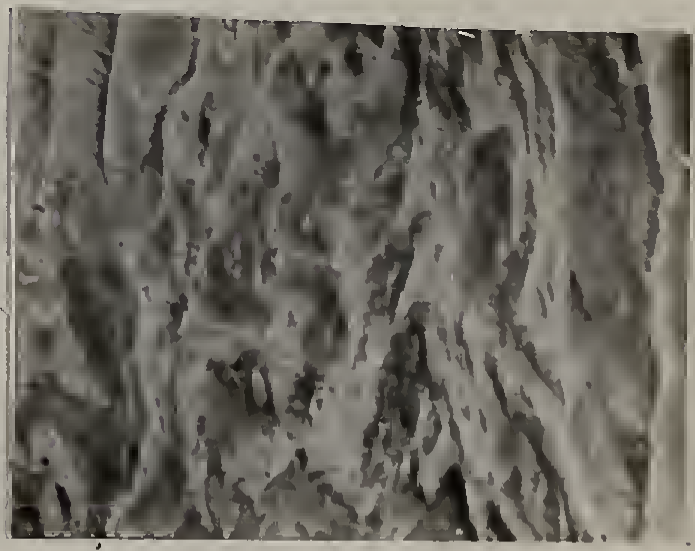

Figure 3.8 TEM images showing (A) bright and (B) dark field images longitudinal sections of fibers composed of 69/31 PBZT/polyamide fiber spun at spin stretch ratio of 1 . 


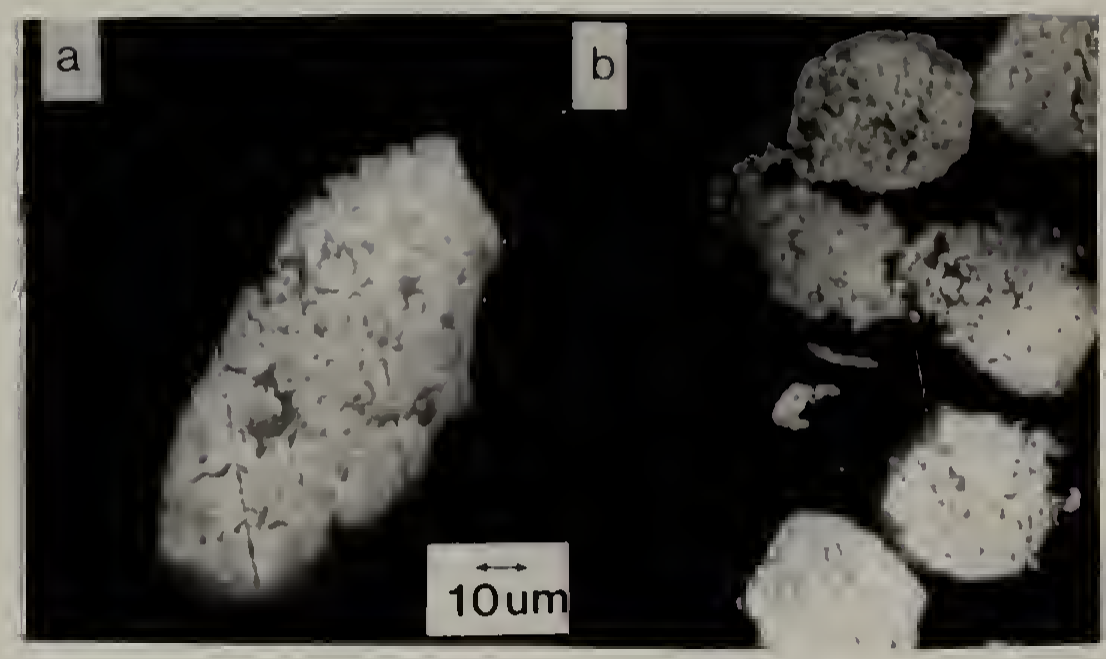

Figure 3.9 Optical micrographs (reflective mode) of cross sections of PBZT/polyamide fibers spun with the following draw ratios: (A) 1 and (B) 4 show the change in diameter, internal structure, and the noncircular cross sections of the fibers. 


\subsubsection{SEM, WAXS and DSC}

Table 3.1 lists the sample fibers and fiber composite produced by E. I. du Pont Inc. for this study. Figure 3.10 shows SEM images of 69/31 PBZT/PEKK fibers before and after ultrasonification. The presence of the PEKK polymer did not inhibit fibril formation. The fiber prior to ultrasonification appeared to have a coating; the fibril structure is revealed after removal of the coating. One would expect this coating to be the coil polymer, which would be the last to coagulate. Evidence for this assumption will be discussed in a Section 3.4. The diameter of the observed fibrils are about 1 micron.

Figures $3.11 \mathrm{~A}$ and $3.11 \mathrm{~B}$ show wide angle $\mathrm{X}$-ray scattering patterns for both the as-spun (AS-10) and heat treated (HT-10) 69/31 PBZT/PEKK fibers. Figure 3.11C is a schematic that labels the reflections. The $\mathrm{X}$-ray patterns indicate that the heat treatment does not enhance the amount of crystallinity of the PEKK compared to the PBZT in the fiber. Higher order reflections of the PEKK are more evident in the as-spun fiber. Given the heat treatment temperature $\left(500^{\circ} \mathrm{C}\right)$ is a considerably higher value than the PEKK crystal melting temperature $\left(332^{\circ} \mathrm{C}\right)$, the amount of crystallinity in the heat treated fiber would depend upon the rate of cooling. Qualitatively, the diffraction spots in the heat treated fibers are sharper suggesting larger crystals.

Table 3.2 and Table 3.3 show the index values of the patterns. The PBZT reflections are indexed with the unit cell from Minter [1982]. The PBZT reflections indicate highly oriented material. The reflections not pertaining to PBZT d-spacings show isotropic orientation. The orientation as indicated by the PEKK reflections is isotropic for both the as-spun and heat treated fibers. There is a significant amount of diffuse scatter indicating the presence of amorphous material.

In the literature there are no reports of a PEKK unit cell. Unit cell parameters for poly(ether ketone) (PEK) and poly(ether ether ketone) (PEEK) have been reported 
(Dawson and Blundell, 1980, Rueda et al., 1983). There are also studies of the crystal habits of PEEK samples with different crystallization conditions (Lovinger and Davis, 1986, Blundell et al., 1989).

A sample of unblended PEKK was melted on a microscope slide by heating to $400^{\circ} \mathrm{C}$ and drawn into fibers with tweezers. After an undetermined amount of drawing and heat treatment at $210^{\circ} \mathrm{C}$, the fiber exhibited crystallinity as shown in the X-ray diffraction pattern in Figure 3.12B. The WAXS diffraction pattern indicates only a small amount of orientation of the PEKK crystals. Figure 3.12A is a X-ray diffraction pattern of the sample of PEKK as received showing a considerable amount of diffuse scatter but no strong crystalline reflections.

Table 3.4 shows the $d$-spacings for the crystalline sample and calculated $d$ spacings for a proposed orthorhombic unit cell. The proposed lattice parameters fit into a series of poly(aryl ether ketone) polymers; Table 3.4 shows the unit cell parameters for comparison. The bond angles between the ether and ketone linkages are equivalent and therefore account for the similarity of the observed unit cell in these polymers [Dawson and Blundell, 1980].

The DSC thermogram in Figure 3.13 confirms that the as-received solvent cast PEKK film did not exhibit any crystallinity. The material crystallizes readily at the $20^{\circ}$ $\mathrm{C} / \mathrm{min}$ heating rate as the endothermic peak at $190^{\circ}$ indicates. The similar area under the crystallizing and melting peaks indicates limited crystallinity in the as received PEKK sample.

Optical microscopy

Figure 3.14 shows reflective optical micrographs of cross and longitudinal sections of the as-spun (AS-10) and heat treated (HT-10) multi- filament PBZT/PEKK fibers. The shape of the area of a fiber's cross section is not round, but irregular. Figure 
3.14A demonstrates that the separate filaments of the fibers may meld together during processing.

The reflective microscopy indicates there are micron size domains within the fibers. One would expect contrast from the difference in the refractive index of the two polymers if they were phase separated.

In the images of the longitudinally sectioned specimens in Figures 3.14B and 3.14D, one can observe elongated domain structures. In the elongated structures along the fiber axis in longitudinal sections, one is attempting to detect a morphology of the size approaching the limits of resolution in optical microscopy. Since the SEM micrographs indicate a fibrillar structure, one would expect the PEKK to be either fibrous or exist as a matrix filling the space between the PBZT fibrils. TEM images of PBZT/PEKK fibers

Figure 3.15 shows TEM bright and dark field images of a cross section of the as-spun 60/40 PBZT/PEKK (AS-10) fiber. On the bright field image an arrow marks the direction of the knife marks and induced tears in the section. Wrinkles that are perpendicular to the knife direction are in both micrographs of the thin section, but are more prominent in the dark field image. An "E" labels the epoxy at the fiber surface. The dark field image, arising from the PBZT [100] and [010] equatorial reflections of the electron diffraction pattern, shows the same mottled structure seen in the bright field image, but with reverse contrast.

The darker regions away from the edge indicate that the PEKK regions are as large as a micron in cross section. There is also evidence of finer structure (about 0.01 micron in size) seen with higher contrast in the dark field image (Figure 3.15B).

Figure 3.16 shows the rectangular area in Figure 3.15A at higher magnification. The material with differing contrast from the fiber interior and the epoxy is evidence of a coating at the fiber and epoxy interface. Cohen demonstrated the microfibrillar network 
of rigid rod polymers by infiltration of water swollen PBZT with epoxy [Cohen and Thomas, 1988]. He speculated upon the random structure of the cross sections of the microfibrils; the image appears to be of such a cross section.

The fabrication of these composite fibers from biphasic solutions evidently yields a coil polymer infiltrated PBZT fibrillar network. During coagulation of the spinning dope, the PBZT solidifies as a fibrillar network swollen with the coil polymer solution, along with the larger domains of the coil polymer evident in Figures 3.15 and 3.16.

Figure 3.17 shows TEM bright and dark field images of longitudinal sections of the heat treated PBZT/PEKK (HT-10). The elongated domains have a fine phase structure similar in width to those in Figure 3.15. The PEKK domain alignment is along a different direction from the arrow indicating the knife direction in Figure 3.17. There are regions of dark contrast in the bright field image (Figure 3.17A) that may be debri resulting from the spinning process.

TEM images of a cross section of a neat PBZT film in Figure 3.18 do not show any of dark areas that are seemingly the minority component PEKK domains.

Discussion of the identification of the different domains will occur in a subsequent section concerning the morphology of composites made from these fibers. 


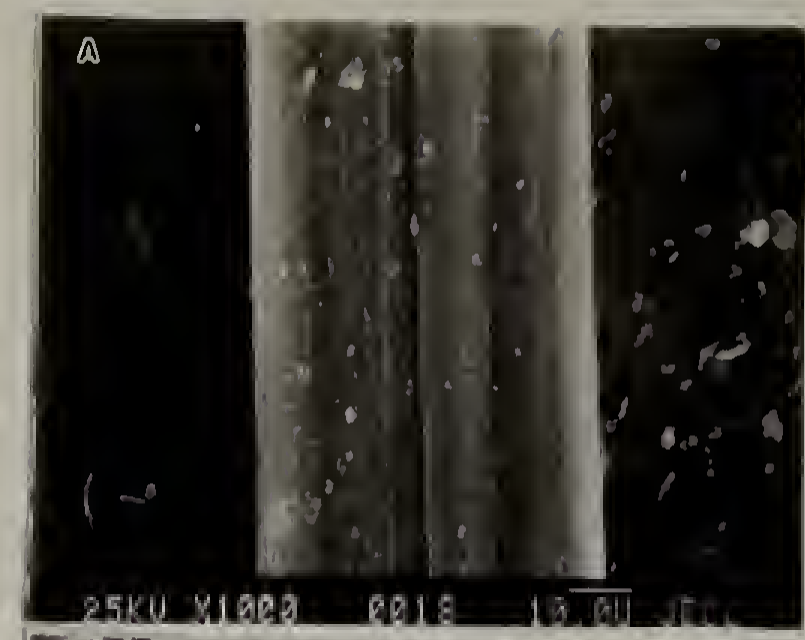

Figure 3.10

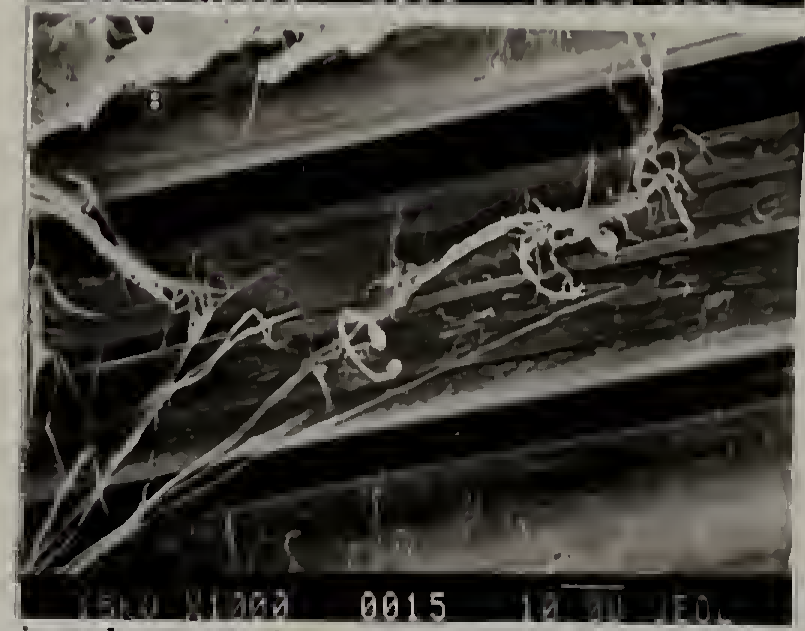

Scanning electron micrographs of PBZT/PEKK fibers (A) before and (B) after ultrasonification indicating that a coating is on the fibers. 

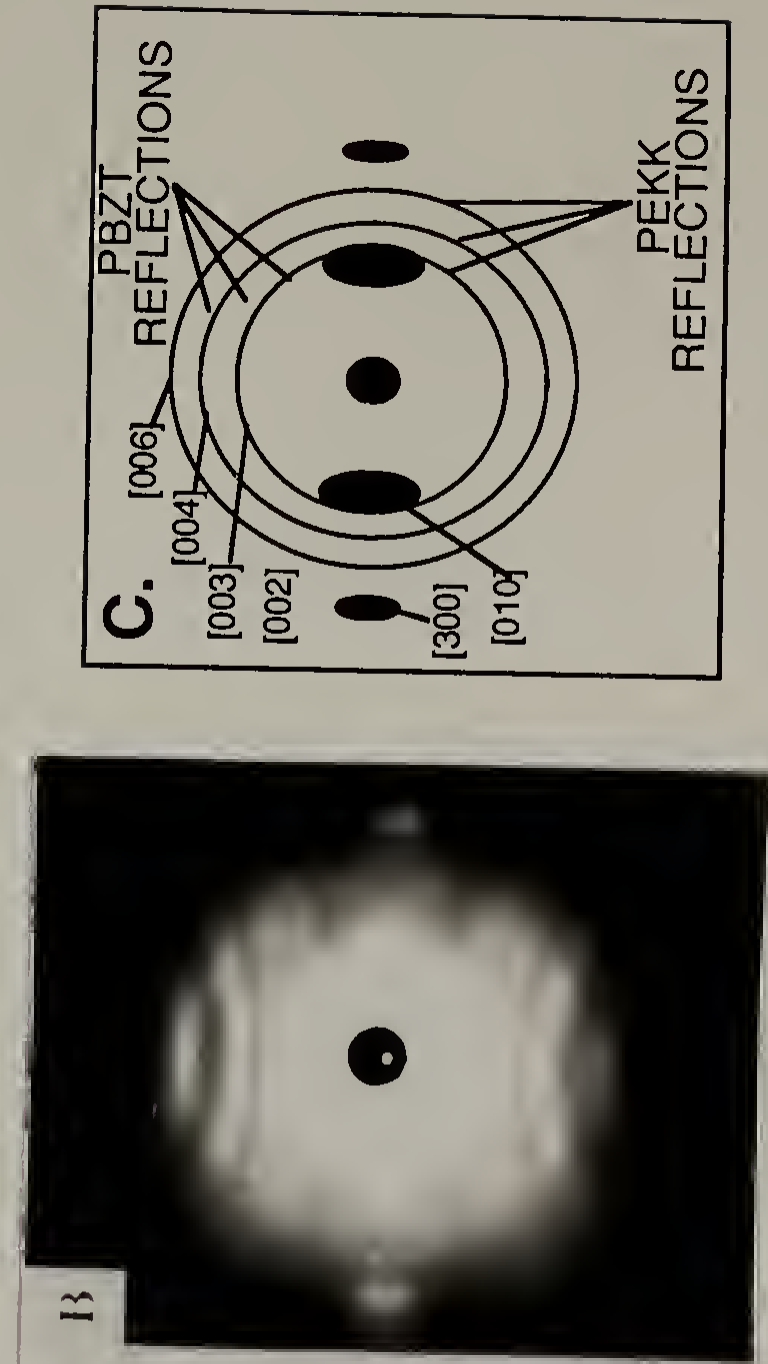

$\stackrel{乛}{F}$

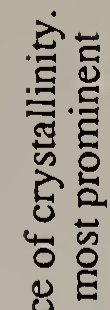

造

仓ั山

ญ. ญ

늠

త్ర

放

के

क्षे

๓ิษ

강

ฮี

돕

牙

Ð

है

बें

इn

도

केत

के

पे

ยี

․ㅗㅇ.5

용

덩

․․․

速氜

궁요

入ㄷํㅇ

语跑

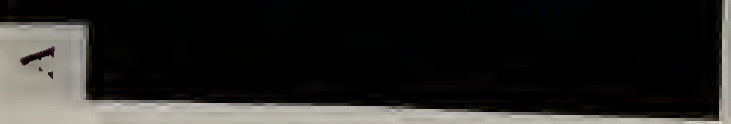

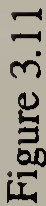




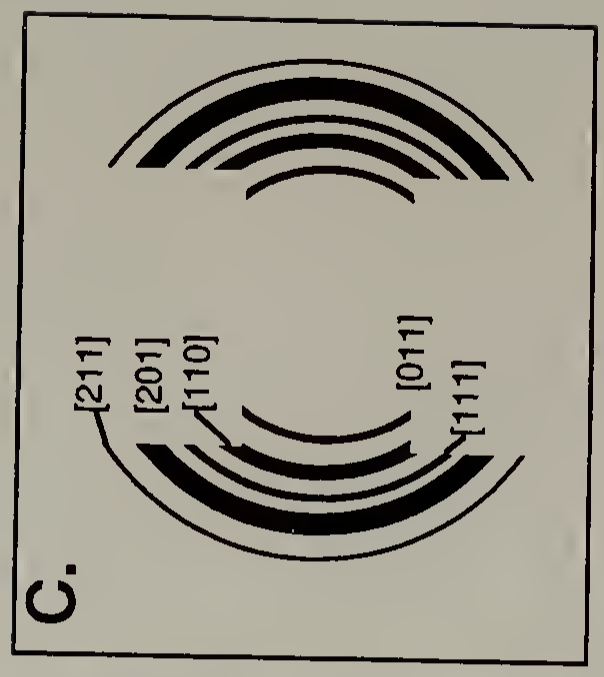

E

ن

ह

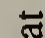

范

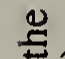

हต

马ี

官诘

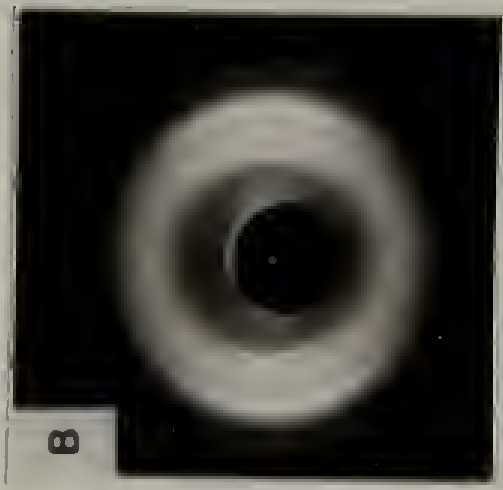

๑ิ년

동

运。

후

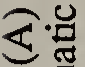

틀

8

$\geq<$

ठ্ঠ

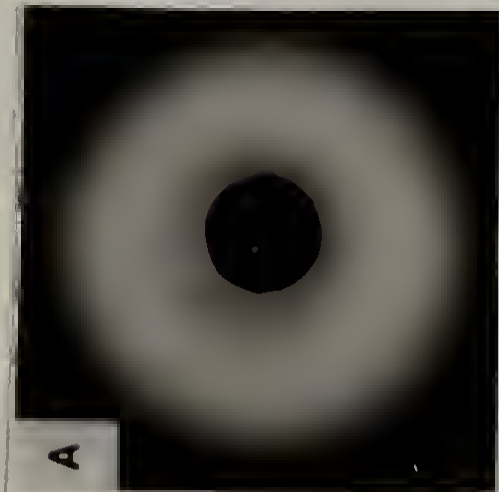

종

证

되.5

a.

4

\&

․․․

苞

ธิต

范

5

흥

突导

골 
Table 3.2

PBZT/PEKK Composite Fiber (PBZT Reflections Only)

Camera Length:

Wavelength:

$17.75 \mathrm{~mm}$

$0.1544 \mathrm{~nm}$

Unit Cell : $\quad(\mathrm{nm})$

$\begin{array}{llll}\text { a: } & 0.597 & \text { alpha : } & (\mathrm{deg}) \\ \text { b: } & 0.362 & \text { beta : } 90 & 90 \\ \text { c: } & 1.245 & \text { gamma: } & 95.2 \\ \text { Error } & = & \mid \Sigma \mathrm{d}_{\text {meas }}-\mathrm{d}_{\text {calc }}= & 0.048\end{array}$

$\begin{array}{lllll}\begin{array}{l}\mathrm{d}_{\text {meas }}(\mathrm{hkl}) \\ (\mathrm{nm})\end{array} & \mathrm{h} & \mathrm{k} & \mathrm{l} & \begin{array}{l}\mathrm{d}_{\mathrm{calc}}(\mathrm{hkl}) \\ (\mathrm{nm})\end{array} \\ 1.25 & 0 & 0 & 1 & 1.25 \\ 0.63 & 0 & 0 & 2 & 0.62 \\ 0.60 & 1 & 0 & 0 & 0.59 \\ 0.42 & 0 & 0 & 3 & 0.42 \\ 0.37 & 0 & 1 & 0 & 0.36 \\ 0.32 & 0 & 0 & 4 & 0.31 \\ 0.20 & 3 & 0 & 0 & 0.20 \\ 0.22 & 0 & 0 & 6 & 0.21\end{array}$

Table 3.3 PBZT/PEKK Composite Fiber (PEKK Reflections Only)

Camera Length: $\quad 17.75 \mathrm{~mm}$

Wavelength:

$0.1544 \mathrm{~nm}$

Unit Cell: $\quad(\mathrm{nm})$

a: $\quad 0.733$ alpha: $(\operatorname{deg}) \quad 90$

b: 0.585 beta: 90

c: $\quad 0.988 \quad$ gamma: 90

Error $=\Sigma \mathrm{d}_{\text {meas }}-\mathrm{d}_{\mathrm{calc}}=0.027$

$\mathrm{d}_{\text {meas }}(\mathrm{hkl}) \quad \mathrm{h} \quad \mathrm{k} \quad \mathrm{l} \quad \mathrm{d}_{\text {calc }}(\mathrm{hkl})$

(nm)

0.18

0.23

0.22

0.27

0.28

0.29

0.33

0.35

$\begin{array}{llll}\mathrm{h} & \mathrm{k} & \mathrm{l} & \begin{array}{l}\mathrm{d}_{\text {calc }}(\mathrm{hkl}) \\ \text { (nm) }\end{array} \\ 1 & 3 & 1 & 0.19 \\ 1 & 2 & 2 & 0.24 \\ 0 & 2 & 3 & 0.22 \\ 1 & 2 & 0 & 0.27 \\ 0 & 1 & 3 & 0.29 \\ 0 & 2 & 0 & 0.29 \\ 0 & 0 & 3 & 0.33 \\ 2 & 0 & 1 & 0.34\end{array}$


Table 3.4

PEKK d-spacings (Melt Drawn Fiber)

Camera Length:

Wavelength: $0.1544 \mathrm{~nm}$

\begin{tabular}{|c|c|c|c|c|}
\hline $\begin{array}{r}\text { Unit Cell: } \\
\text { a: } \\
\text { b: } \\
\text { c: }\end{array}$ & $\begin{array}{l}(\mathrm{nm}) \\
0.733 \\
0.585 \\
0.988\end{array}$ & \multicolumn{2}{|c|}{$\begin{array}{l}\text { alpha: } \\
\text { beta: } \\
\text { gamma: }\end{array}$} & $\begin{array}{r}\text { (deg) } \\
90 \\
90 \\
90\end{array}$ \\
\hline \multicolumn{5}{|c|}{ Error $=\Sigma \mathrm{d}_{\text {meas }}-\mathrm{d}_{\text {calc }}=0.017$} \\
\hline $\begin{array}{l}\mathrm{d}(\mathrm{hkl}) \\
\text { meas. } \\
(\mathrm{nm}) \\
0.59 \\
0.50 \\
0.46 \\
0.42 \\
0.34 \\
0.30 \\
0.25\end{array}$ & $\begin{array}{l}0 \\
0 \\
1 \\
1 \\
2 \\
2 \\
0\end{array}$ & $\begin{array}{l}1 \\
1 \\
1 \\
1 \\
0 \\
1 \\
0\end{array}$ & $\begin{array}{l}0 \\
1 \\
0 \\
1 \\
1 \\
1 \\
4\end{array}$ & $\begin{array}{c}\mathrm{d}(\mathrm{hkl}) \\
\text { calc. } \\
(\mathrm{nm}) \\
0.59 \\
0.50 \\
0.46 \\
0.41 \\
0.34 \\
0.30 \\
0.25\end{array}$ \\
\hline
\end{tabular}

Proposed Unit Cells of Poly(aryl ether ketones) all orthorhombic)

Polymer nit cell parameters $(\mathrm{nm})$ :

$\begin{array}{lccc}\text { PEK* } & \text { a } & b & c \\ \text { PEEK* } & 0.76 & 0.60 & 0.10\end{array}$

$\begin{array}{llll}\text { PEEK* } & 0.78 & 0.59 & 0.10\end{array}$

$\begin{array}{llll}\text { PEEK ** }^{*} & 0.78 & 0.59 & 0.99\end{array}$

*Dawson and Blundell

**Lovinger and Davis 


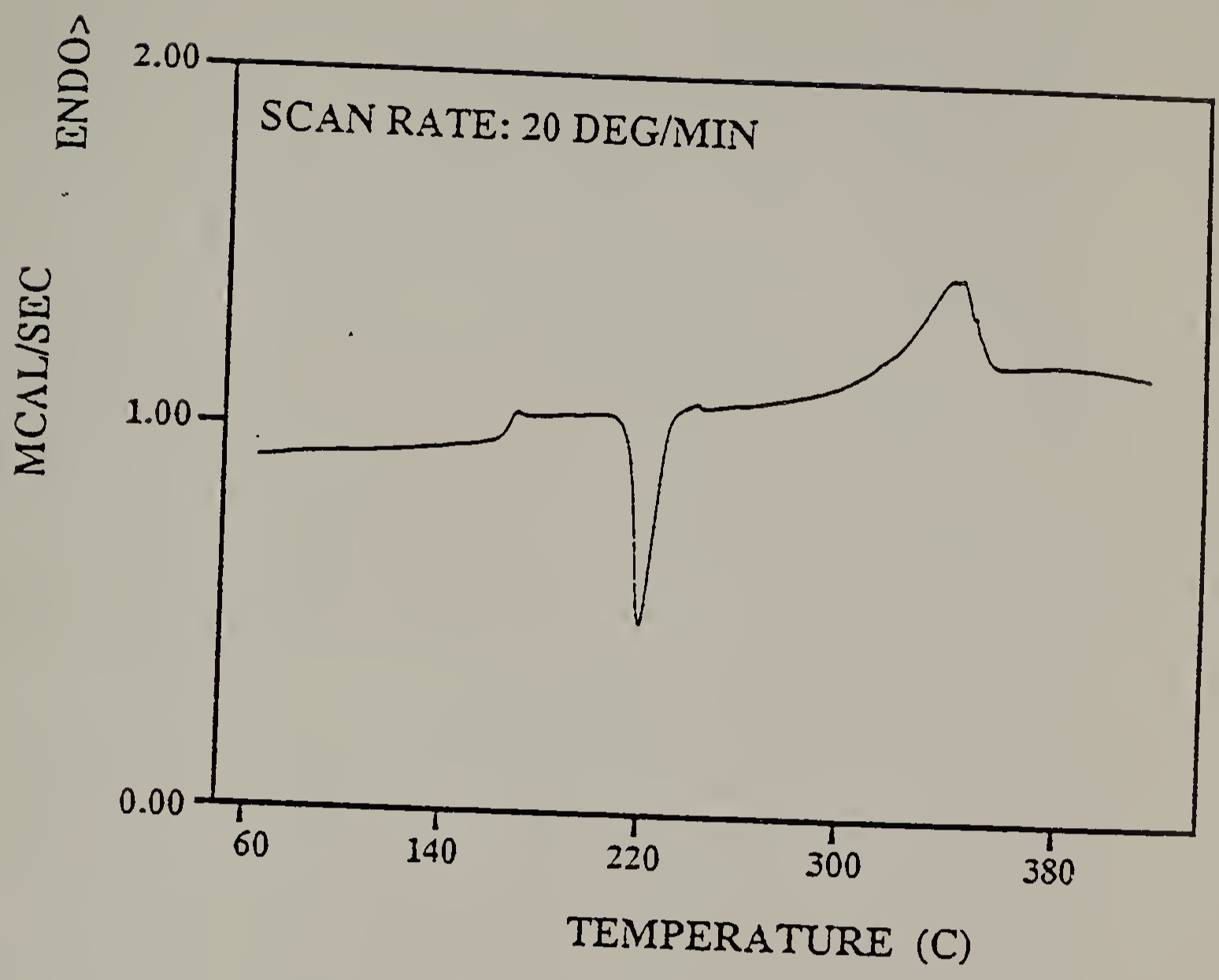

Figure 3.13 Differential scanning calorimetry scan of the as received PEKK polymer sample. The material crystallizes readily at the $20^{\circ} \mathrm{C} / \mathrm{min}$ heating rate. The similar area under the crystallizing and meling peaks indicates limited crystallinity in the as received PEKK sample. 
Figure 3.14 Optical micrographs of sectioning block showing (A) cross and (B) longitudinal sections of fibers composed of $60 / 40$ PBZT/PEKK (AS-10). Optical micrographs of the PBZT/PEKK fibers after heat treatment (HT-10) showing (C) cross and (D) longitudinal sections. One observes structure on the micron scale. 


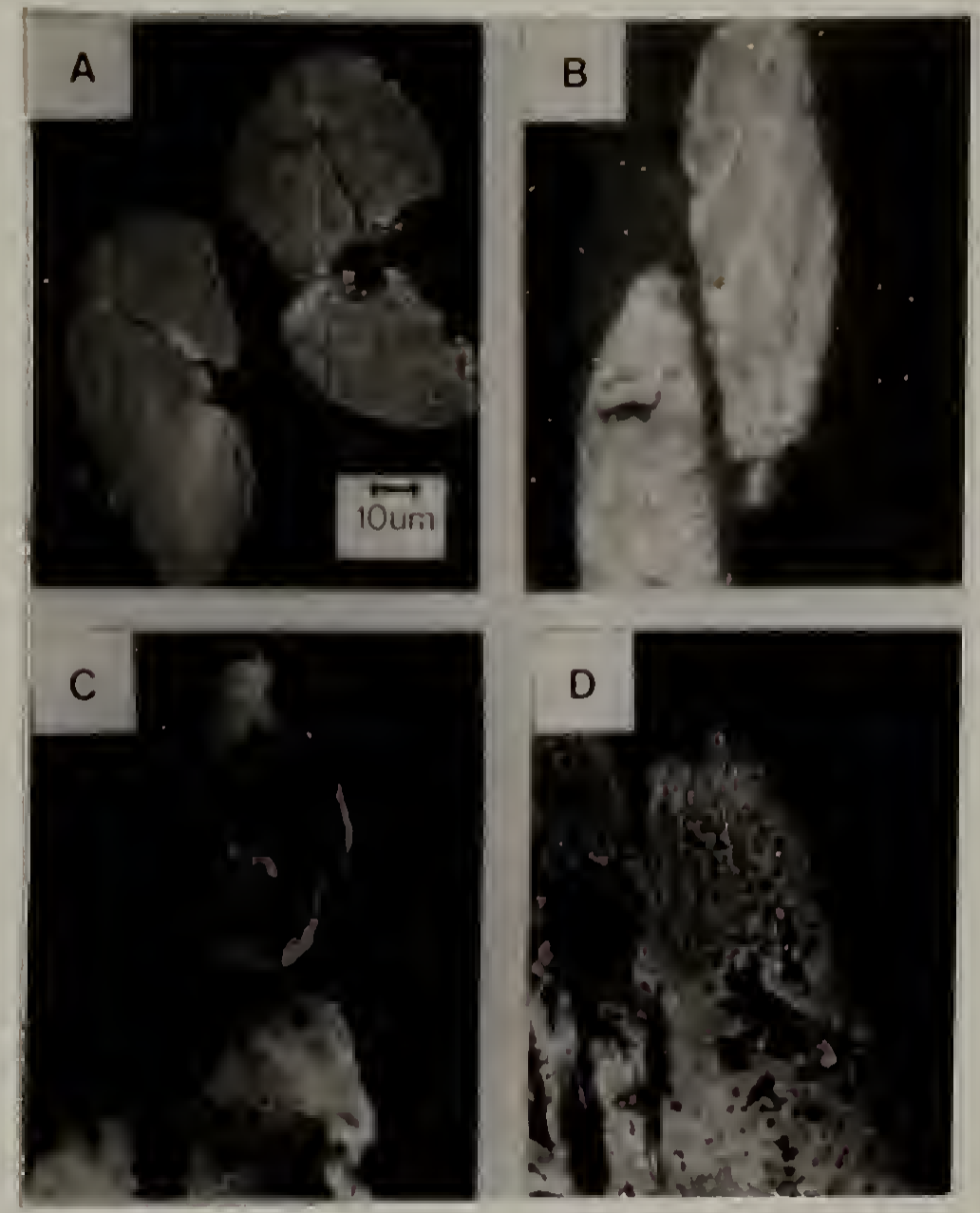




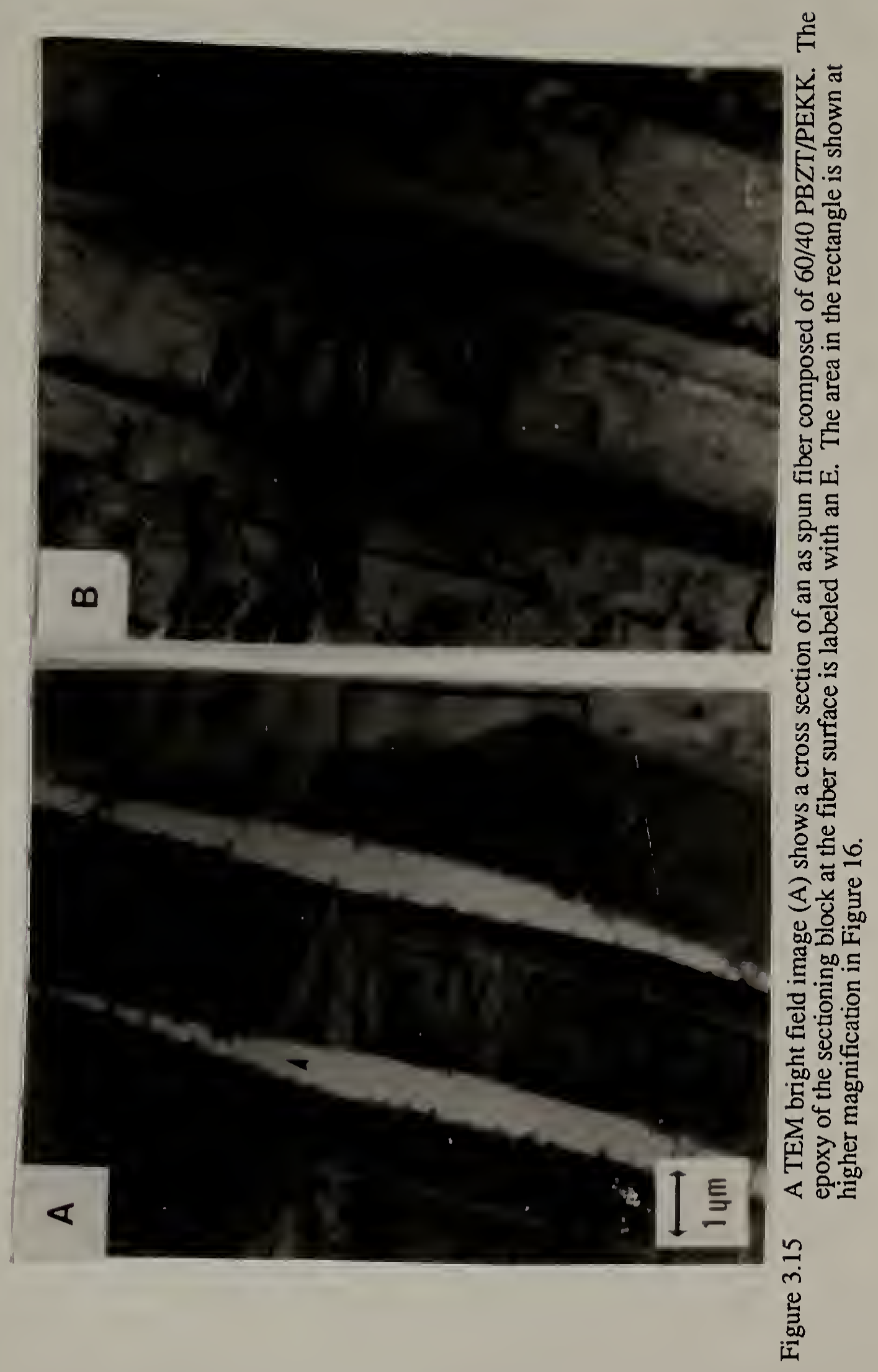




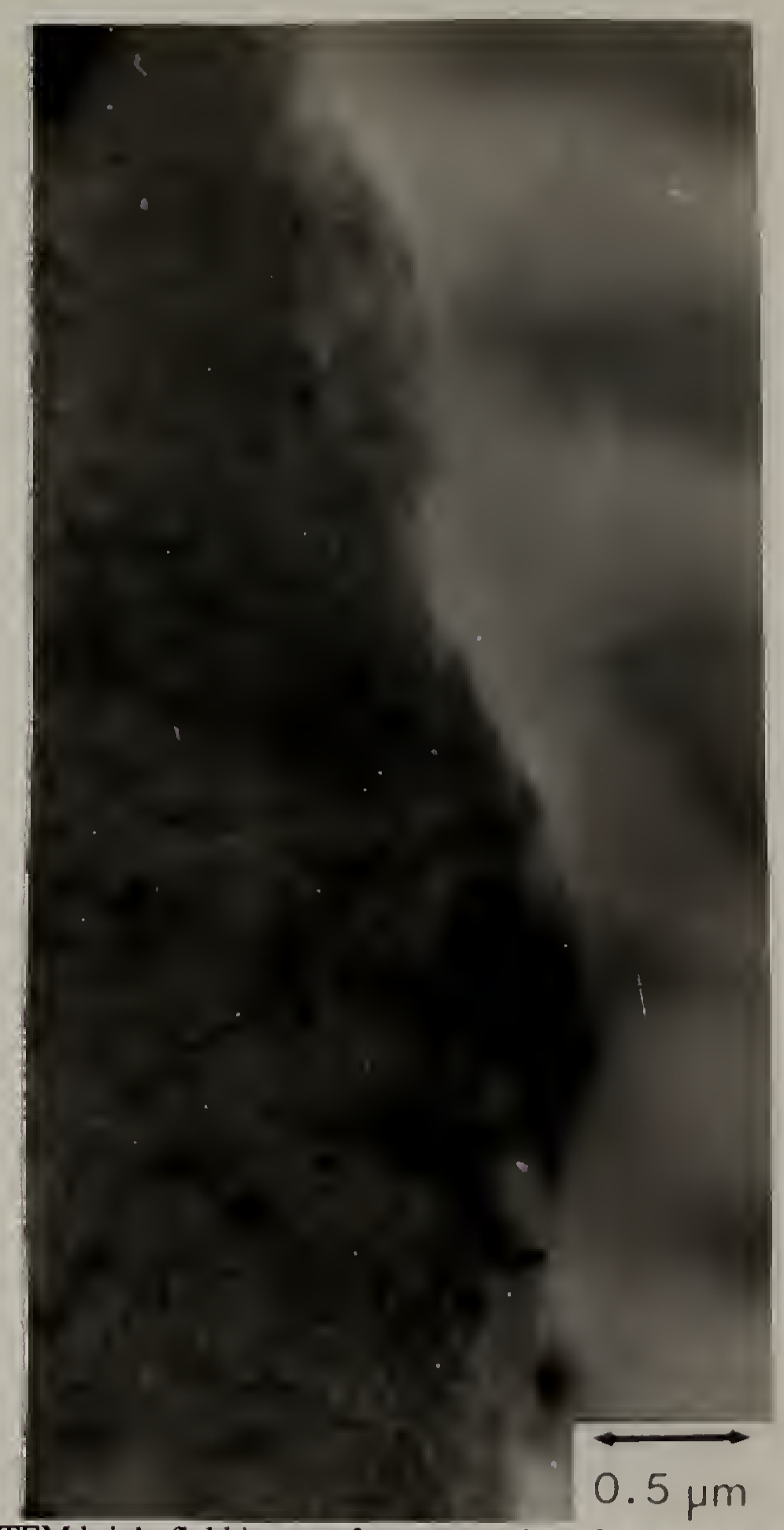

A TEM bright field image of a cross section of an as-spun fiber composed of 60/40 PBZT/PEKK at higher magnification in which the fine phase structure suggests a cross section of the microfibrillar network observed by Cohen. 


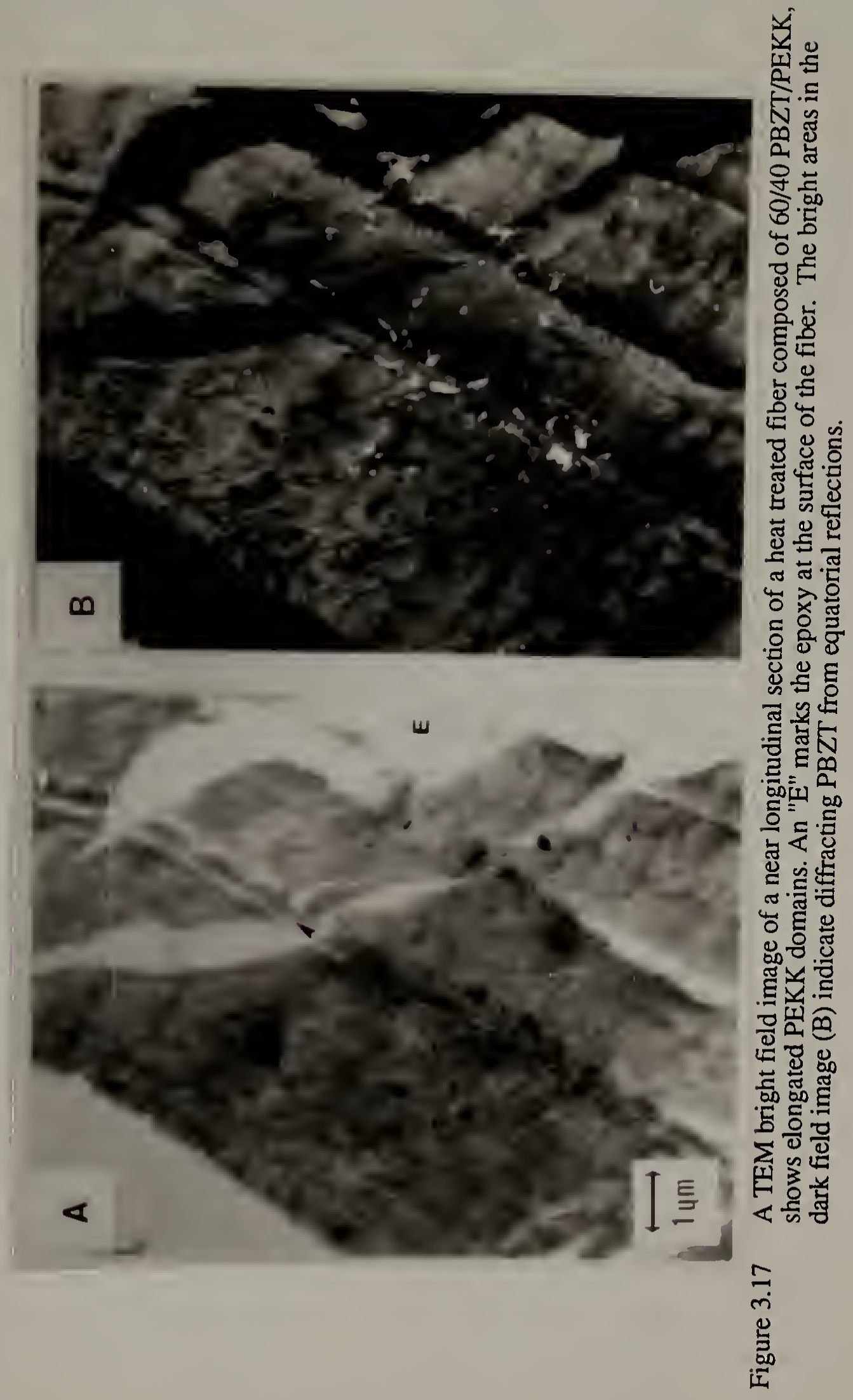




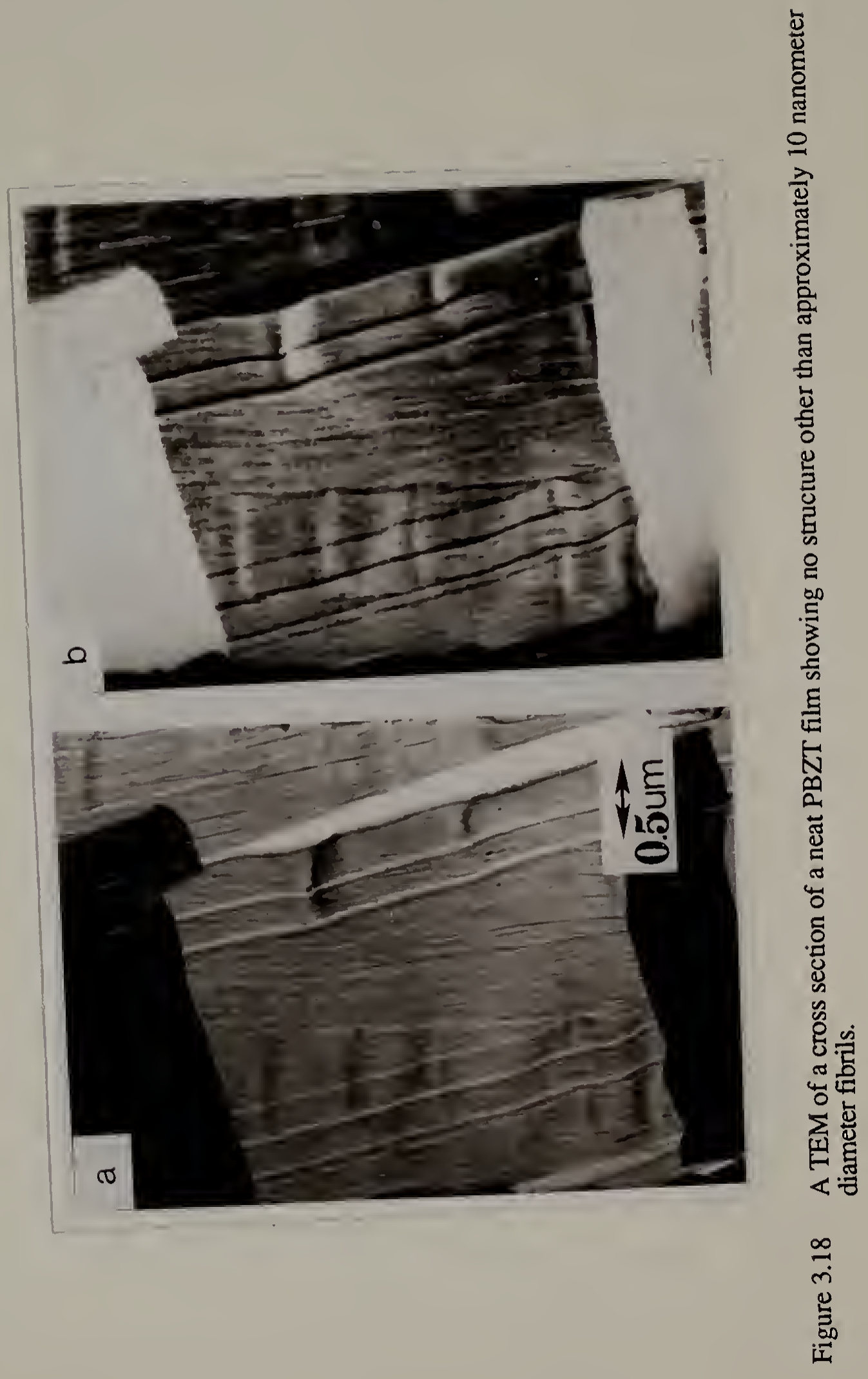




\subsubsection{Consolidating the composite fibers}

Figure 3.19 is a schematic demonstrating the formation of a composite structure from the fibers spun from biphasic solution. For a pressure and heat treatment to consolidate the fibers into a composite structure, there must be sufficient coil polymer on the surface or the coil polymer in the interior must be forced to the exterior. There appears to be considerable coil polymer dispersed within fibers on a micron and submicron length scale, but there is no preference for migration of the coil polymer to the surface. Typical autoclave conditions for hot pressing of the fibers into composite are 4000 psi and $400^{\circ} \mathrm{C}$.

Due to the ability to infiltrate water swollen microfibrillar networks with epoxies and polymer solutions, one could with good confidence assume the three dimensional interconnectivity of the coil polymer regions on a submicron range [Cohen, 1987, Hwang, 1989]. However, for the larger domains, such an assumption about a continuous morphology is more speculative. In fibers made with low draw ratios (see Figure 3-6) where the domain structure is more evident, the coil polymer appears to disperse in elongated domains, as one would expect from the structure of spinning dope (see Figure 3.5) and the elongational flow during spinning.

The fiber has a two phase structure on two approximate length scales; one at the micron level and the other at the one-hundredths of a micron level. Assuming the domain structures have an approximate cylindrical geometry (i. e. $R=0.5$ um and $R=.005$ um), the respective PBZT/coil polymer interfacial surface area per cubic centimeter of material would be $4 \times 10^{4} \mathrm{~cm}^{2}$ and $4 \times 10^{6} \mathrm{~cm}^{2}$. The larger phase size is the result the competition between forces due to the interfacial surface energy of the two polymer solutions and the viscous forces during the mixing and elongational and shear 
deformation during processing. The smaller size is an indication of the formation of the microfibrillar network of PBZT.
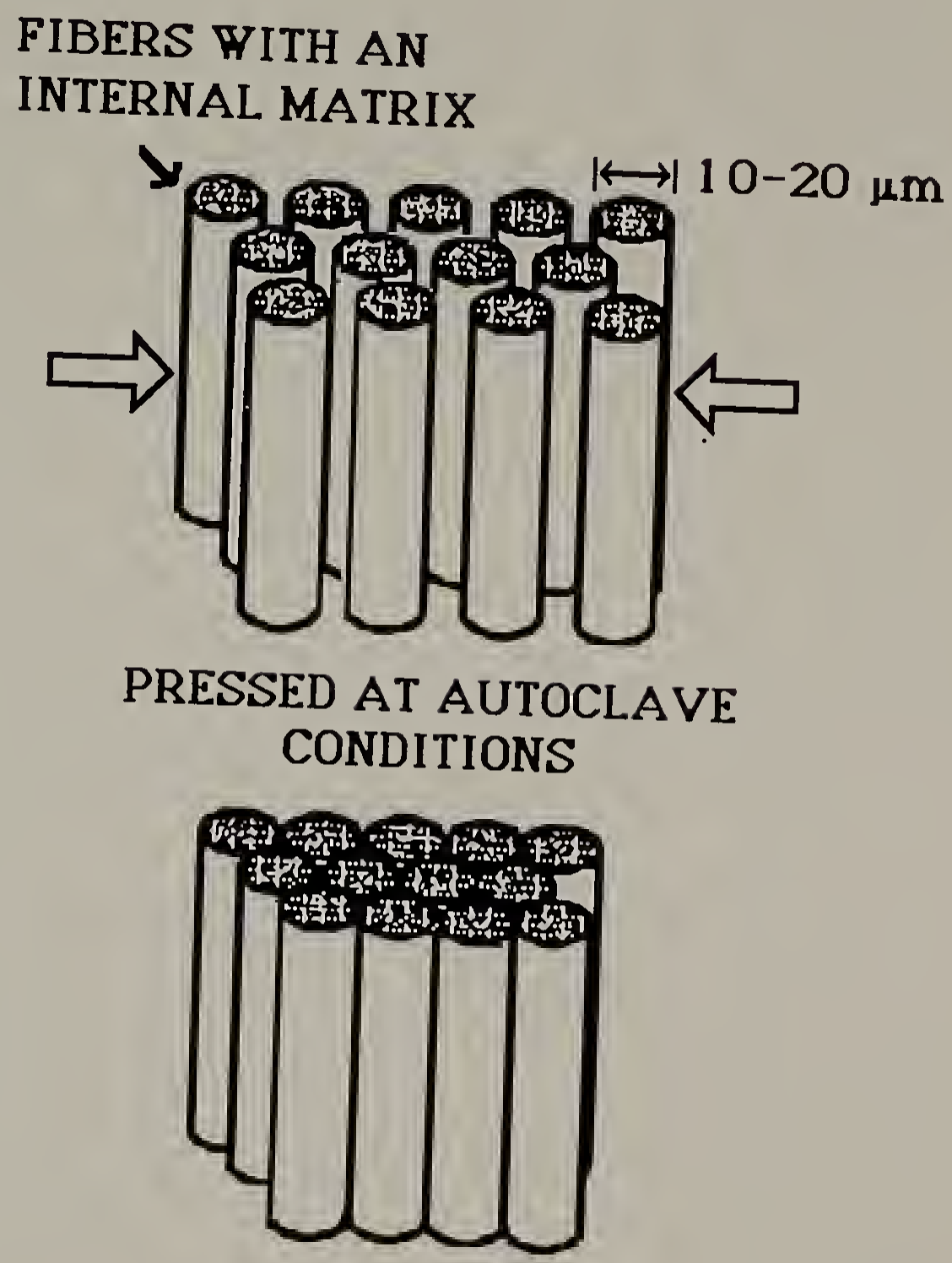

Figure 3.19 Schematic of composite formation in composites.

Once the oriented fiber morphology is set and the fiber is taken above the melt temperature of the coil polymer, there is no practical method of applying force to the system to create a pressure gradient to force the melted component to the surface. The 
fiber also appears to deform in cross section to relieve any applied external stress, so it is apparent that the only coil polymer that is available for consolidation is at or near the surface upon coagulation.

One method of generating the desired pressure gradient is to treat the fiber with a solvent (such as $\mathrm{H}_{2} \mathrm{SO}_{4}$ ) in which the coil polymer is soluble but the PBZT is not. The osmotic pressure due to the swelling of PEKK or polyamide would cause it to either extrude through some path to the surface, or burst the fiber open.

The following sections discuss the morphology of composites made by consolidating the two phase fibers by a variety of methods .

\subsubsection{Tape from PBZT/PEKK fiber}

Figure 3.20 shows a reflective optical photomicrograph of a microtomed cross section of a tape specimen (TA-10) composed of as spun 60/40 PBZT/PEKK fibers. The individual outlines within the cross section suggest the shapes of the fibers are not strictly cylindrical, but conform to fill the volume into which the fibers are pressed. The size of the individual fibers observed in the SEM micrograph in Figure 3.10 are the same as the size of cross section shapes observed in the pressed tape (TA-10). In Figure 3.20 , the sharp division between fibers seen may be due to physical separation during microtomy or the presence of a higher PEKK concentration at the interface between fibers.

Figure 3.14 shows that the as-spun and heat treated fibers also exhibit irregular, non circular fiber cross sections indicating a collapse in structure upon coagulation. The fiber does not have the rigidity to maintain a circular cross section upon the application of external pressure while the coil polymer domains are in solution during coagulation or in the melt during heat treatment. 
Figure 3.21 shows TEM bright and dark field images of thin sections taken from the embedded TA-10 specimen shown in Figure 3.19. The TEM bright field image in Figure 3.21A shows PEKK cross sectional structure within the fiber before pressing is the same as that observed within the fibers of the TA-10 tape. The sections exhibit wrinkles and tears incurred during the microtoming. The micrographs display a domain structure with sizes on the order of 1 micron. Figure 3.21A shows the PEKK regions in the micrographs appear darker than the PBZT material. This may be a result of phase contrast which would result in a contrast reversals with differences in specimen thickness and density.

There is an electron diffraction of the image area in Figure 3.21B and Figure 3.21C. Upon deformation during microtoming, the PBZT reorients in the direction of the knife marks which is typical when sectioning PBZT [Cohen and Thomas, 1988].

Obtaining dark field TEM images by selecting different locations on the electron diffraction pattern with an aperture helps identify the composition of the domains. Figure 3.21C is a TEM dark field using the PBZT [010] and [100] reflections as demonstrated on the Selected Area Electron Diffraction (SAED) pattern in the inset. The PEKK has reflections within this range and exhibits a great amount of diffuse scatter that the aperture does not block when centered on the PBZT equatorial reflections; the PEKK domains have the same average brightness as the PBZT domains. The dark field background is visible at the surface tears. As with the fiber samples, the appearance of the microtomed PBZT areas appears distinct from the PEKK domains.

Figure $3.21 \mathrm{C}$ is a dark field of the same area with the aperture placed just off the main beam forming the image with diffuse scatter mostly from the PEKK. The contrast of the image reverses; PEKK areas appear brighter than the PBZT domains. The PEKK regions are by far the most distinct and show a much finer detail. 
It is not evident whether the observed torn areas in the sections occur between fibers or within the fibers themselves. The separated sections in Figure 3.20 and the SEM images in Figure 3.10 indicate that the individual fibers easily break into fibrils that are one micron in size.

The diffracting PBZT in Figure 3.21B again indicates the fine internal structure of the individual fibers. This fine structure is a result of the coagulation step where the PBZT fibrillar network precipitates first with the remaining volume being filled with the coil polymer solution which subsequently precipitates. The bright areas in Figure 3.21C indicate that there is considerable diffracting PEKK at the surface of the fibers. The manufacturing of the tape did not cause an observable difference in the shape of the domains; the PEKK does not appear to flow out of the fiber. The lack of physical integrity of the tape confirms this observation.

\subsubsection{Morphology of PBZT/PEKK Tensile Bar}

Figures 3.22A and 3.22B show optical micrographs obtained in the reflective mode of a cross section and a longitudinal section of the fabricated tensile bar from consolidation of PBZT/PEKK fibers. Thin sectioning the epoxy embedded composite yields a smooth block face that facilitates observation by reflective optical microscopy. The outlines of the fiber boundaries are evident throughout the section as observed in TA-10 tape sample in Figure 3.20. In the longitudinal section the space between fibers approaches 5 to 10 microns due to the oblique cutting angle. The optical micrographs suggest some internal structure in the fiber, but one must view the TEM images for more detailed information.

Figure 23 shows a TEM bright field image and a TEM dark field images of a ultramicrotomed thin section from the specimen shown in Figure 3.22. Again, in some areas the adhesion between fibers was not enough to keep the fiber surfaces bonded 
during the microtoming process. The dark field image from PBZT reflections (Figure 23B) shows a very fine texture not apparent in the bright field image in Figure 23A.

The PEKK domains within the fibers and at the fiber surface appear lighter in the dark field image using the diffuse scatter (Figure 23C). The PEKK domains show a slightly different contrast and less mottled texture than the PBZT does after sectioning. The coil polymer is still in fine domains and has not been forced to the surface by the hot pressing. The hot pressing process is not sufficient to generate a pressure gradient to induce the melted polymer to the surface of the fiber. .

Figure 24 shows TEM bright and dark field images of longitudinal sections of MB-10. There are rather large PBZT crystallites (about 0.1 microns in size) which appear as dark spots in the bright field image in Figure 24A. A few of the large crystals that are strongly scattering into the aperture appear as white specks in the dark field image. There was not much evidence of PEKK crystallinity in the electron diffraction pattern of the cross section shown in the inset of Figure 23, but the diffuse scatter of the PEKK domains was enough to minimize the observed contrast between the different domains in Figure 23A. The small magnitude of diffraction from the PBZT in the cross section view occurs because of the limited orientation induced in the PBZT during sectioning. The lack of orientation reduces the number of PBZT crystallites, ones not resolvable in this case, that scatter out of the objective aperture for the bright field image or into the objective aperture for the dark field image.

The TEM images of longitudinal section in Figure 24A and Figure 24B have much greater contrast between the PBZT and PEKK domains because of a greater number PBZT crystallites oriented in the Bragg condition scattering into the objective aperture in the dark field image. The images show the alignment of the PEKK domains is in the fiber direction and their size is less than a micron in width. 


\subsubsection{PBZT/Polyamide composites}

Figure 25 shows the effect of acid treatment on a PBZT/PA fiber. Figure 25a is a cross section of an untreated 10 micron cross section fiber. The fibers were run through concentrated sulfuric acid (which could dissolve the polyamide but not the PBZT) and recoagulated with water. Upon acid treatment the fibers form bundles that have a significant amount polyamide between them as shown in Figure 25b. The fibers also have irregular cross sections due to the acid treatment. The longitudinal section of the acid treated fiber in Figure $25 \mathrm{C}$ shows the fibers have elongated internal structure.

Figure 24 is an optical cross section of a PBZT/PA composite consolidated from acid treated tape. The fiber boundaries are less evident than in the composite cross section shown in Figure 25B, but the fiber cross sections of 10 to 20 microns are still evident.

The TEM bright and dark field images in Figure 27 also indicate that the fiber interfaces are well adhered for it is difficult to locate the full circumference of a particular fiber, though torn places in the section do indicate the presence of such fiber interfaces. The visible PEKK domains are about fractions of micron in size, which makes them difficult to discern in the optical micrograph.

\subsection{Composite properties}

Figure 28 compares the modulus and strength properties of conventional epoxy or PEKK composites reinforced with carbon fiber and PBZT fibers and a composite fabricated from in-situ blended PBZT/ PEKK fibers. (The data is from Du Pont). In Figure 28A, the values of the tensile and flex moduli of the PBZT/PEKK composite are comparable to the moduli values of the PBZT/epoxy. The advantage of the PBZT/PEKK composite is the absence of a lay-up step to make the composite. 
The same conclusion can be made for the strength properties of the composites. Unfortunately, the poor compressive and flex strength characteristic of rigid rod polymers also show no difference for the in-situ fiber composites. The carbon fiber (AS4 and T300) composites show much higher compressive strengths than the PBZT fiber composites. One may surmise that the shear modulus of the PBZT fibers has not increased with existence of a nanometer scale PBZT microfibrillar network interspersed with coil polymer.

\subsection{Summary}

The data thus far suggest the overall structure of the tape made from the PBZT/PEKK composite fibers can be characterized by the schematic in Figure 29. The fibers in the tape conform their cross section to the surroundings without appearing to extrude any significant amount of PEKK from their interiors.

The individual fibers are composed of fibrils that are on the order of one micron in diameter with PEKK filling the spaces between the fibrils. The micron size structure is a result of the mixing prior to extrusion and the elongation flow during fiber spinning. There is evidence of submicron features that are a result of precipitation of the PBZT prior to the flexible polymer during coagulation.

The irregular cross sectional shape of the as-spun individual fibers indicates the fibers are pliable in cross section when the coil polymer is swollen with solvent during fiber fabrication. The coil polymer distributes on two length scales within the fiber. The coil polymer is in micron size elongated domains and dispersed on a nanometer sized regions in the microfibrillar PBZT network formed during coagulation. The crystallites of the coil polymer show isotropic molecular orientation in the as-spun fiber and after heat treatment. 
There is sufficient coil polymer on the surface of the as-spun fibers to instill some adhesion when they are heat pressed to make composites. However, the majority of the coil polymer is still in the interior of the fiber. The fiber cross sections contort to fit in the area of the composite cross section when they are heated above the coil polymer melting point, which is advantageous during composite lay-up procedures but makes it difficult to force the coil polymer to the surface of the fibers where it will facilitate adhesion. The acid treatment of fibers decreases the visibility of the fiber boundaries in the consolidated fiber composite.

Composites made with the in-situ fibers show similar properties to conventional composites 


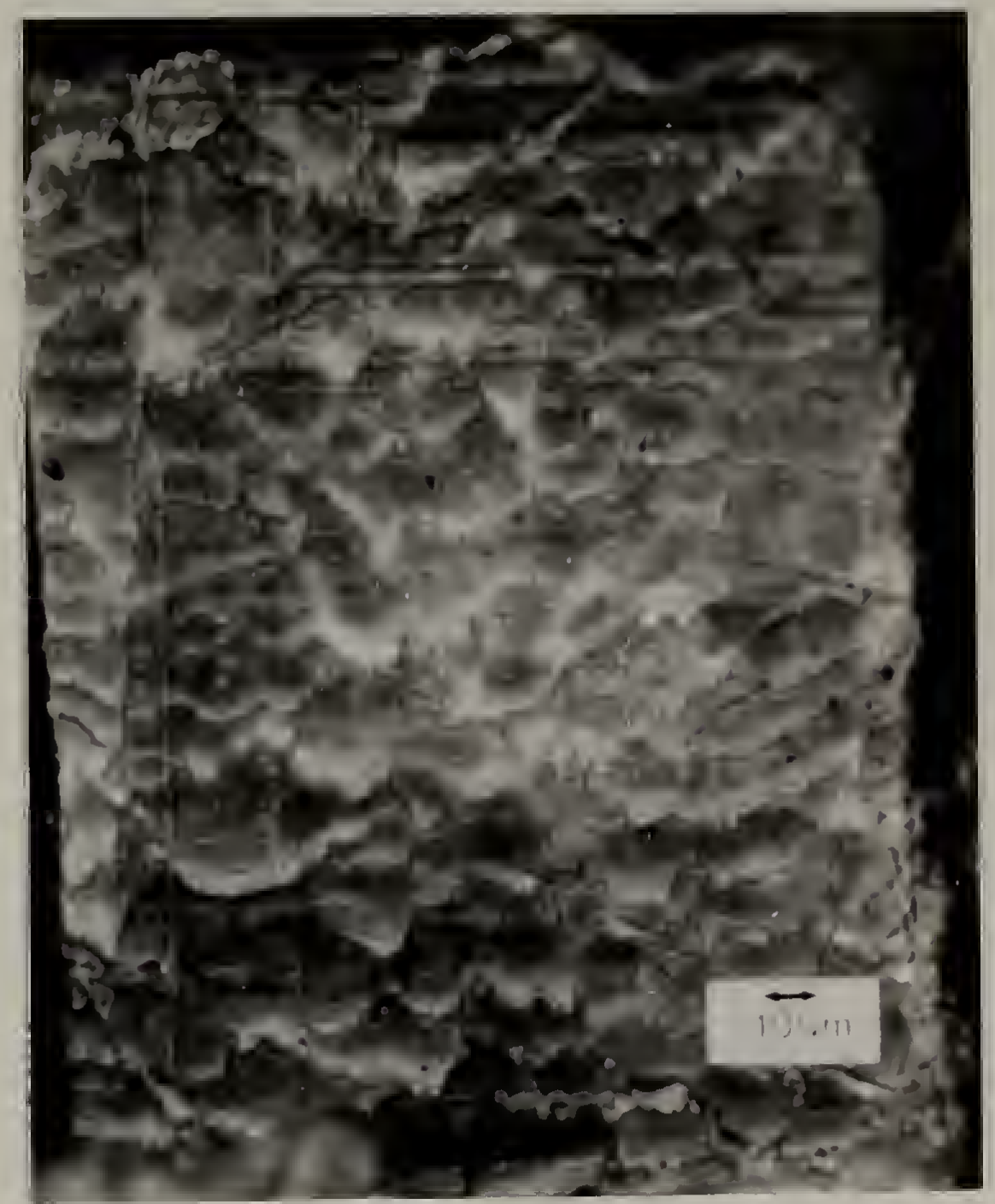

Figure 3.20 An optical micrograph (reflective) of a microtome sectioning block showing a cross section of a tape fabricated by pressing $60 / 40$ PBZT/PEKK fibers. The image shows the outlines of individual fibers and their internal structure. The fiber cross sections deform to fill the space of the cross section. 


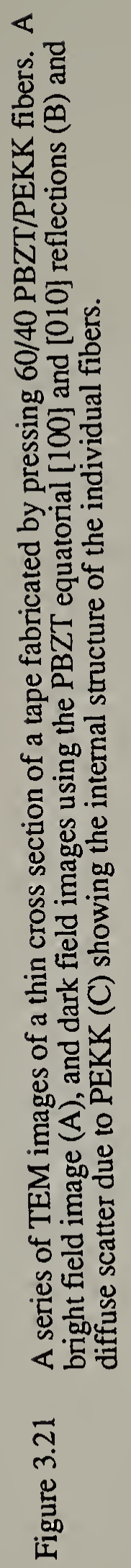




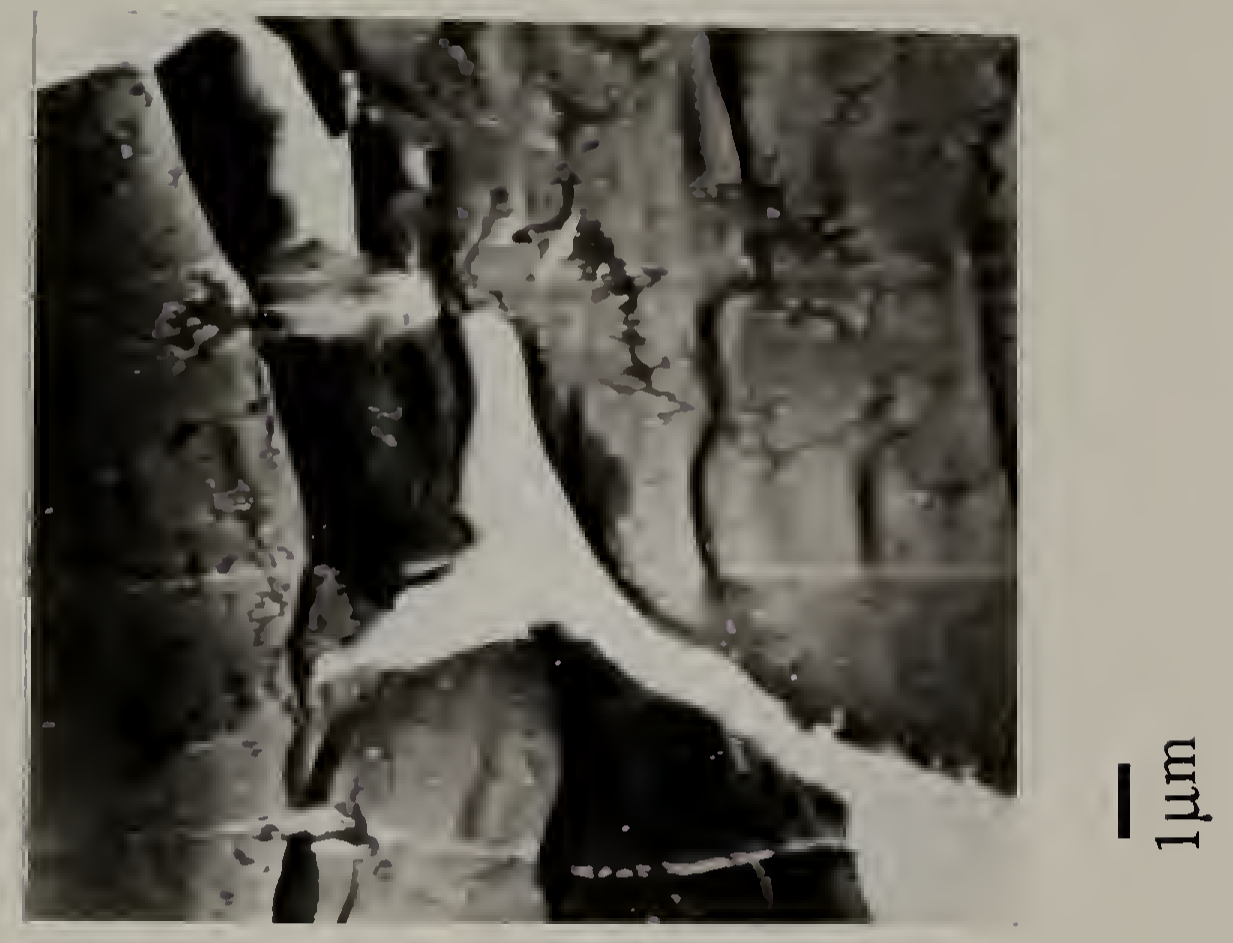

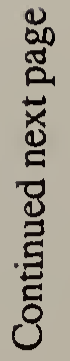




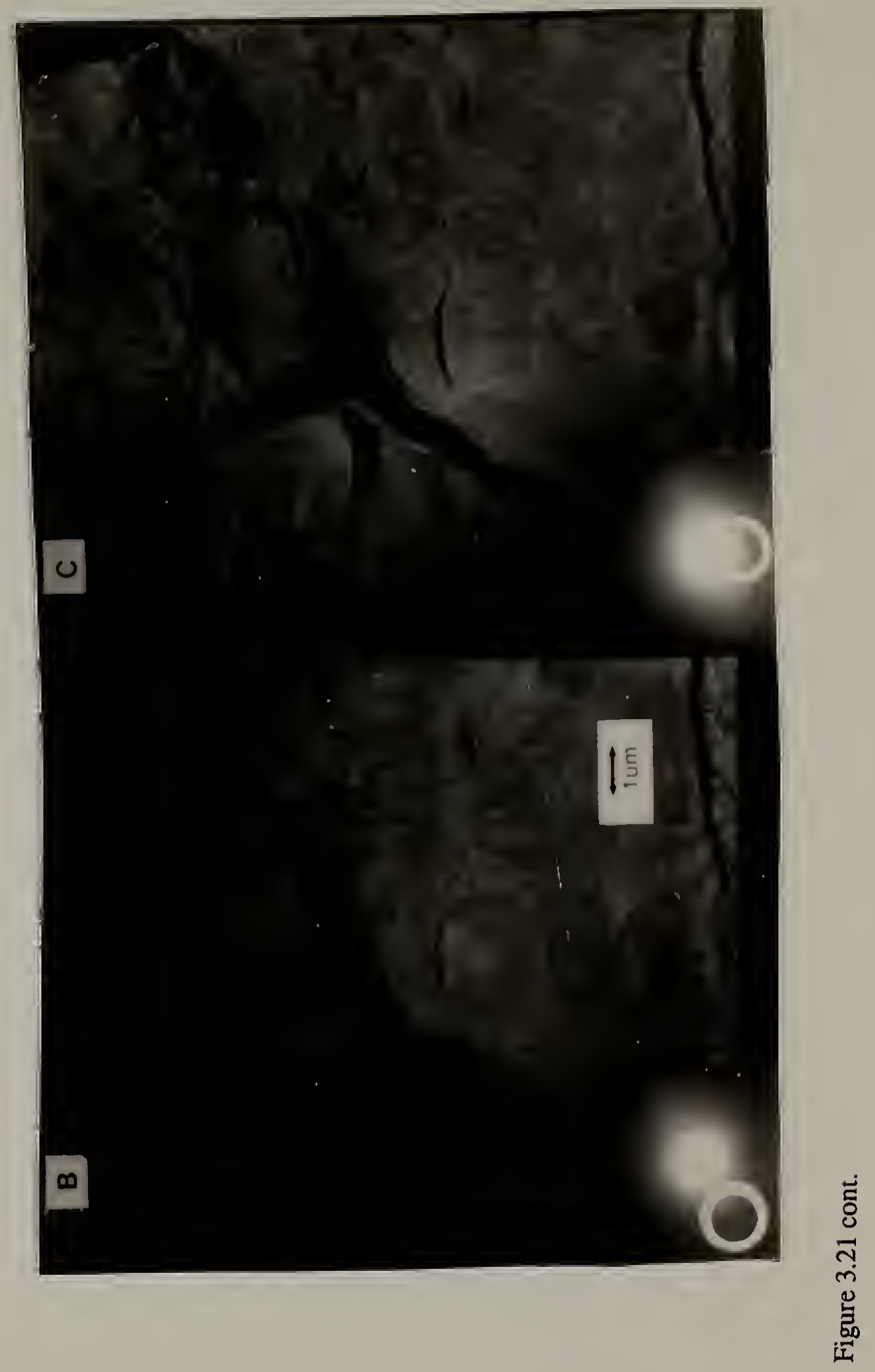




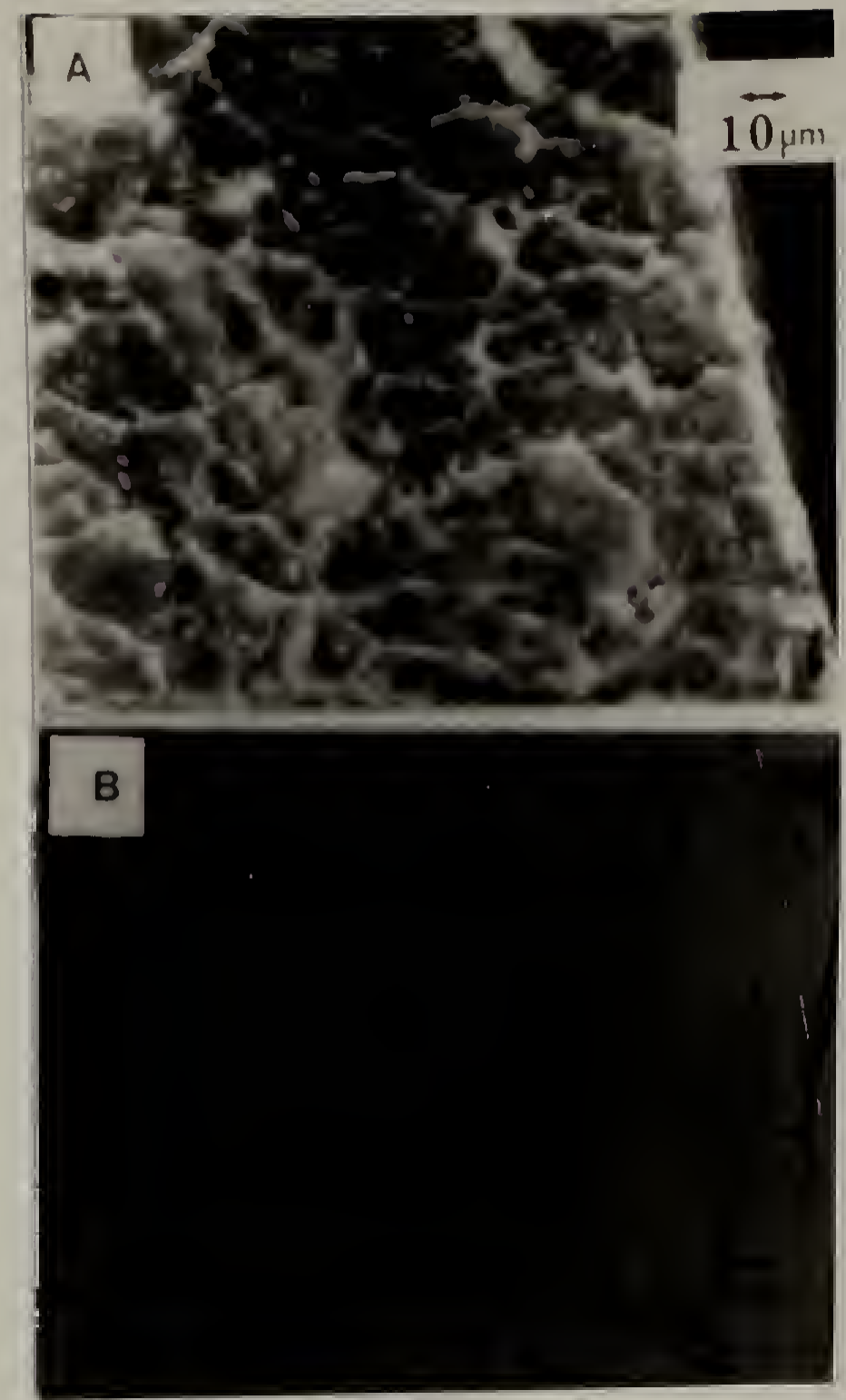

Figure 3.22 An optical micrograph (reflective) of microtome sectioning block showing a cross section (A) and a longitudinal section (B) of a composite fabricated from the PBZT/PEKK fiber tape (MB-10). 


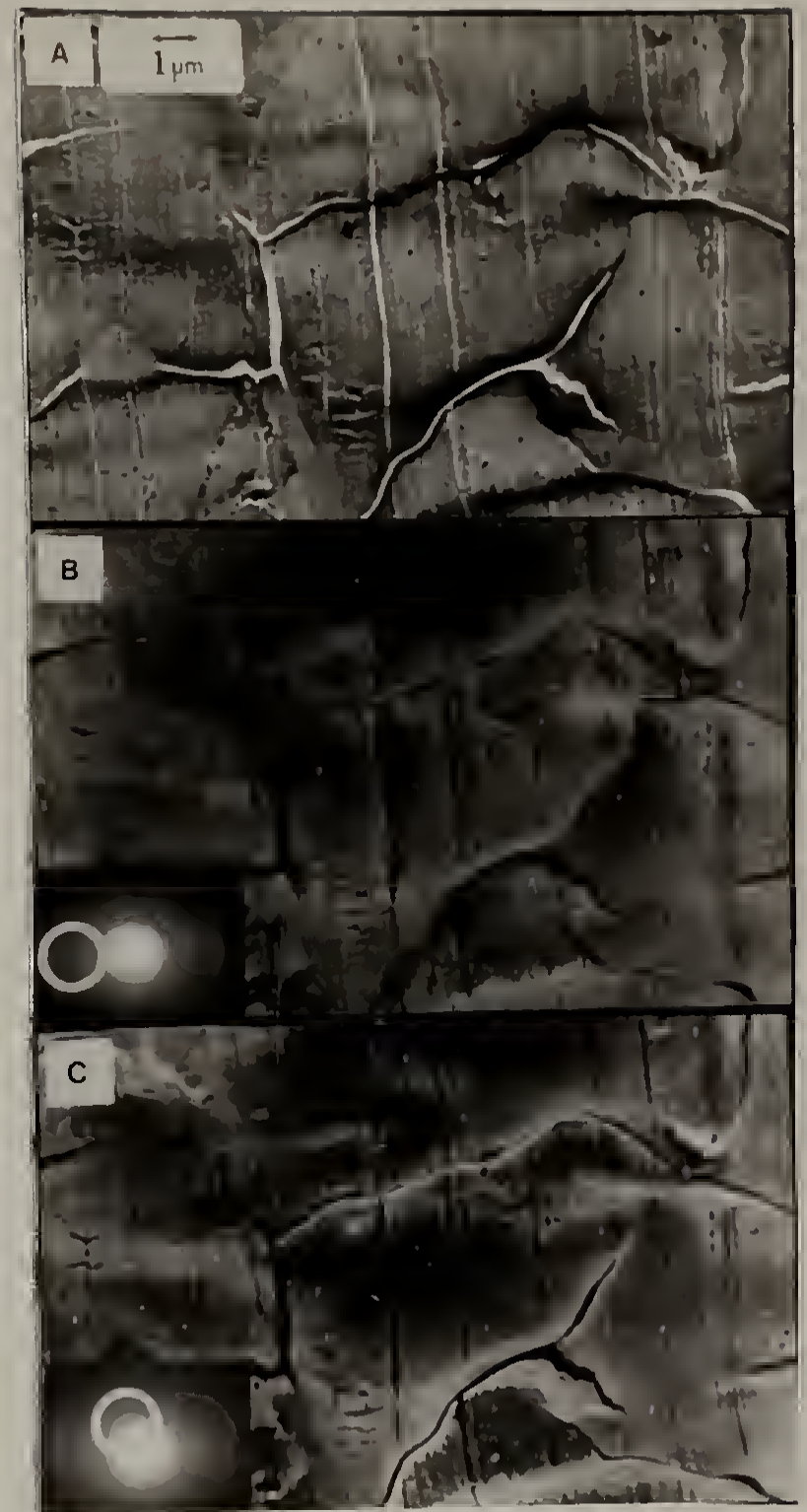

Figure 3.23 A bright field image (A), and dark field images, $(B$ and $C$ ) using the PBZT equatorial [100] and [010] reflections (B) and diffuse scatter (C) due to PEKK of a PBZT/PEKK composite cross section of sample TA10. 


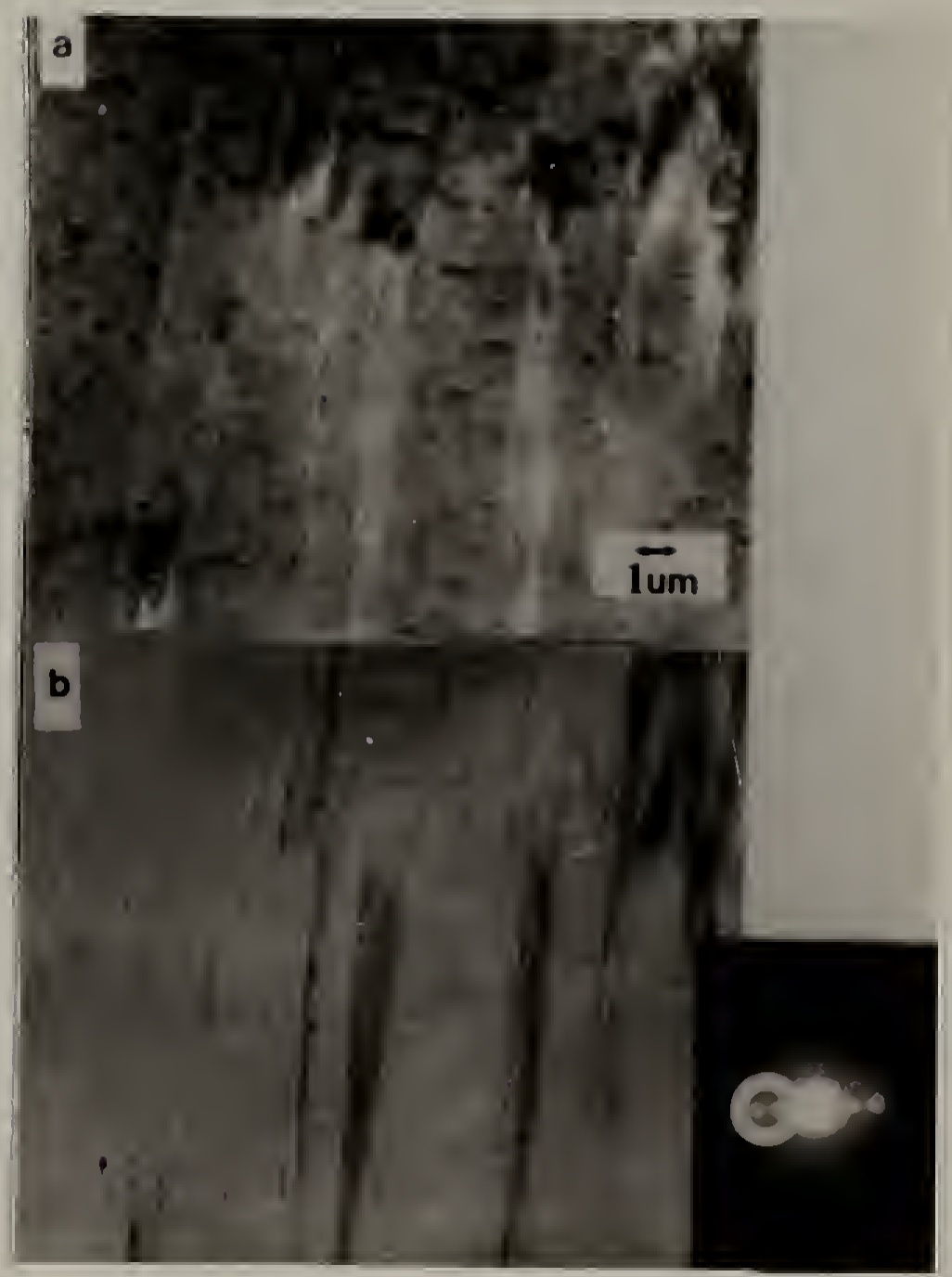

Figure 3.24 A bright field image (A), and dark field images using the PBZT equatorial $[100]$ and $[010]$ reflections $(B)$ of a near longitudinal section from PBZT/PEKK fiber composite MB-10. 
Figure 3.25 An optical micrograph (reflective) of microtome sectioning block showing cross sections of (A) as-spun PBZT/polyamide fiber and (B) acid treated fiber and (C) longitudinal section of the acid treated fiber. 


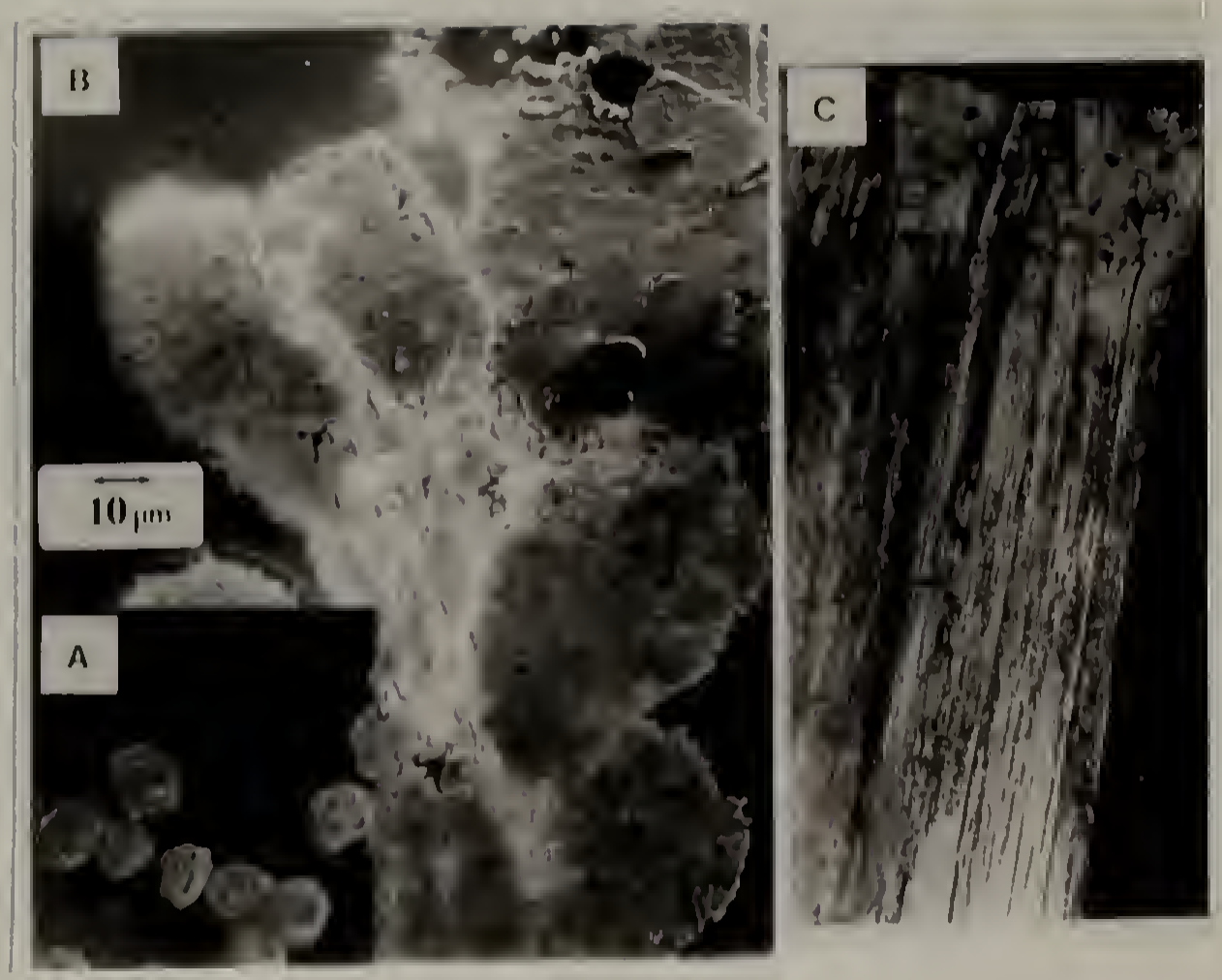




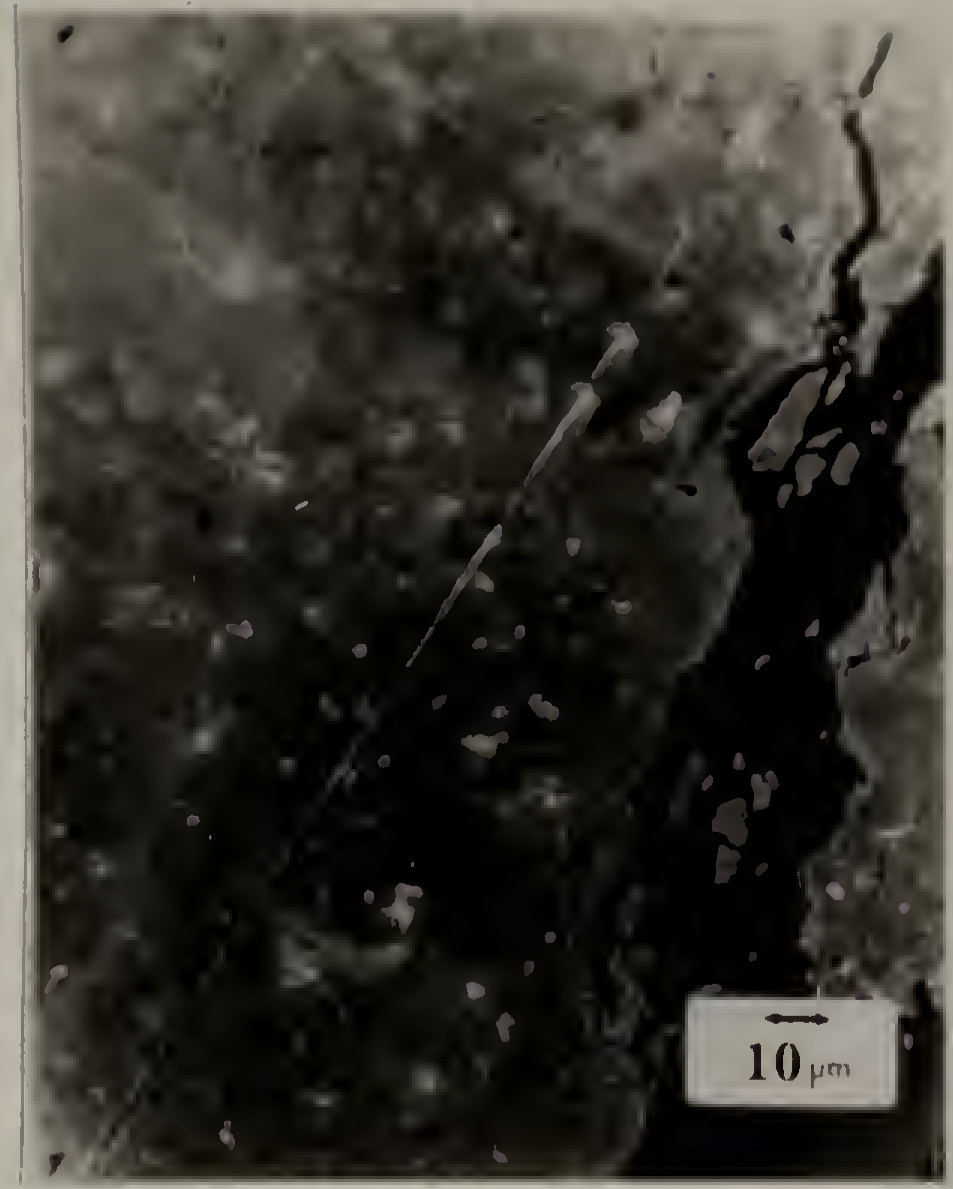

Figure 3.26 An optical micrograph (reflective) of microtome sectioning block showing a cross section of a composite bar fabricated from a PBZT/polyamide acid treated fiber. The outlines of the individual fibers are not as distinct. 


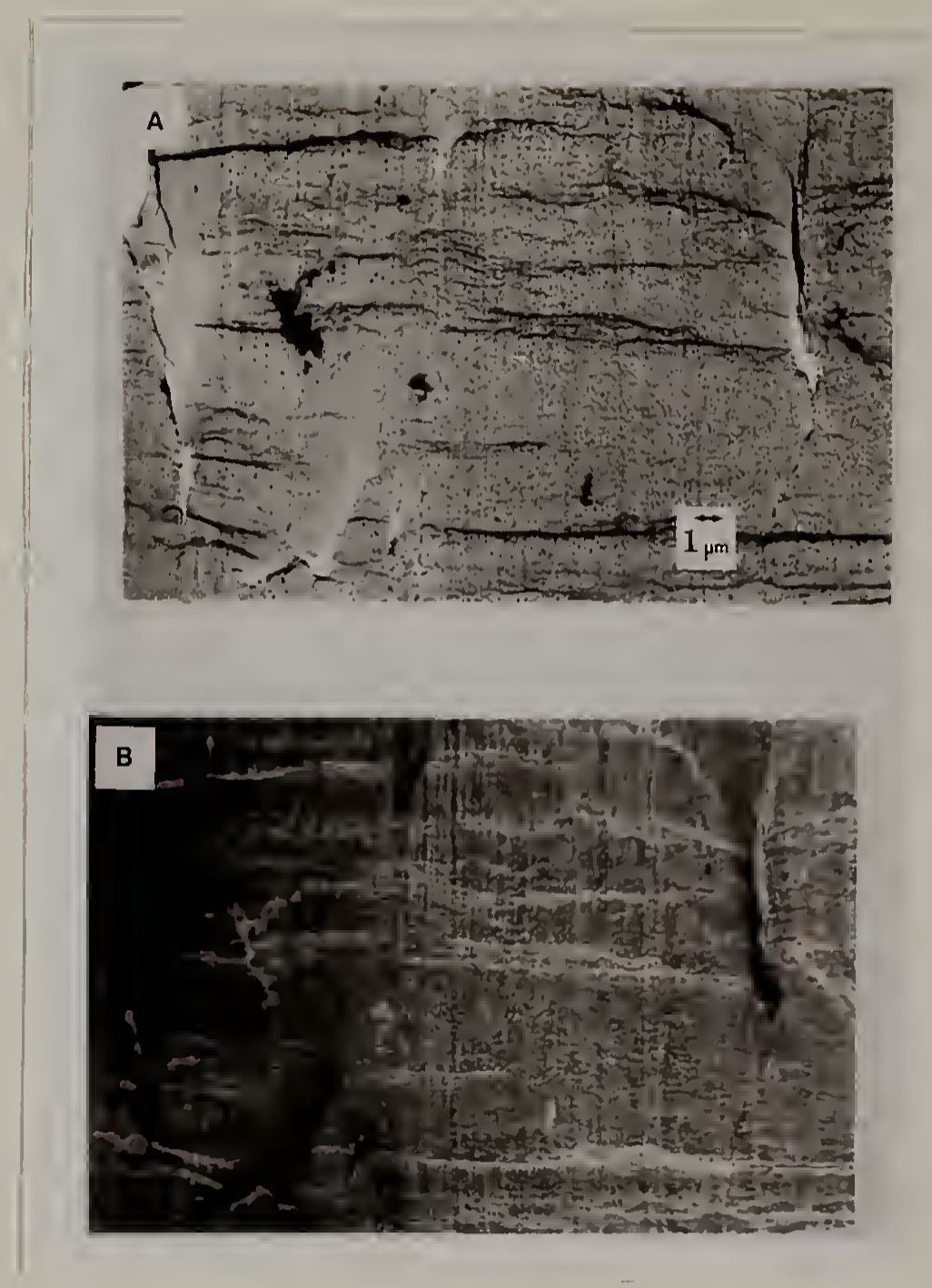

Figure 3.27 A bright field image (A), and dark field image (B) using the PBZT equatorial [100] and [010] reflections of a cross section from a composite fabricated from acid treated PBZT/polyamide fibers. 

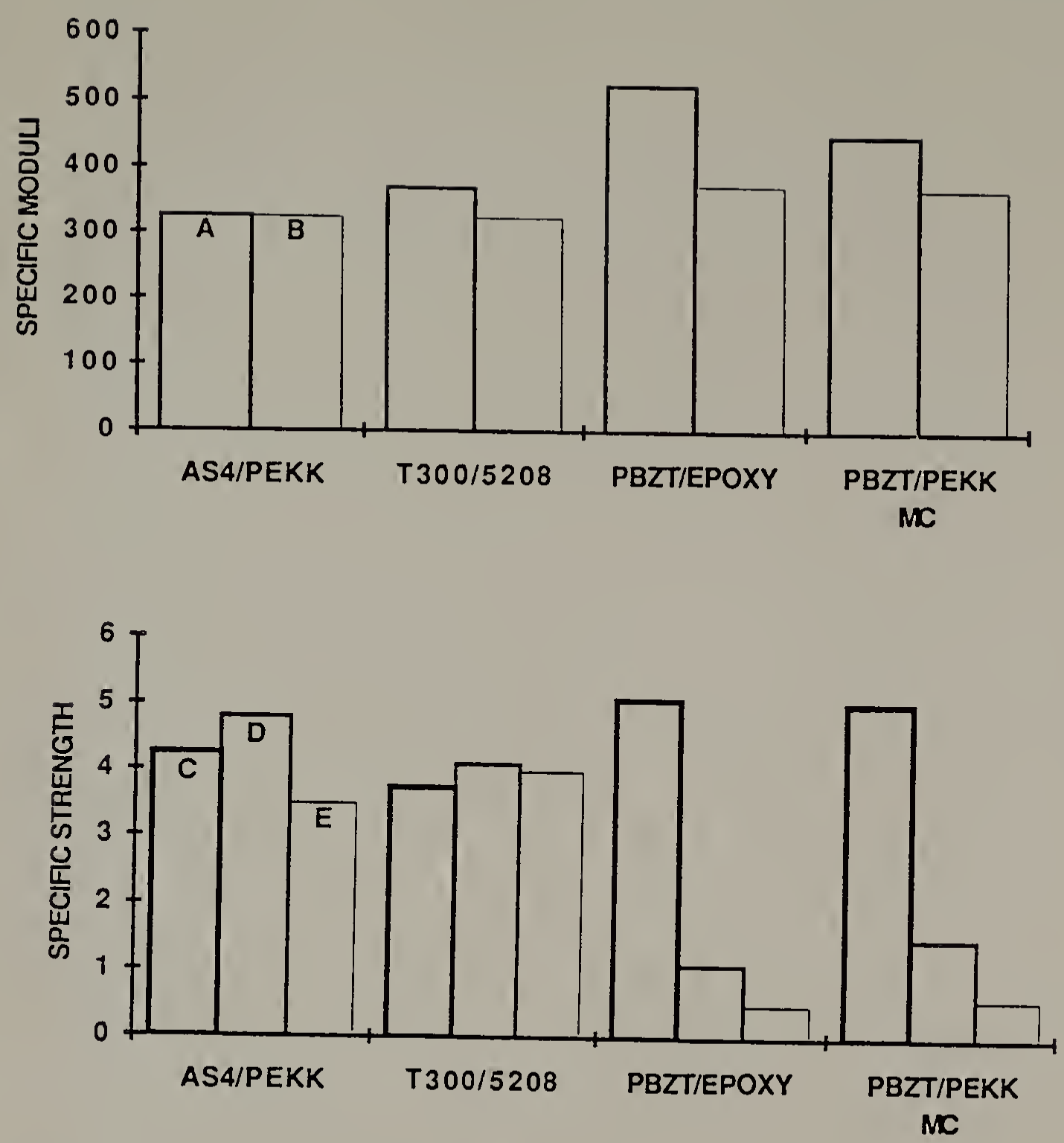

Figure 3.28 (A) Specific tensile, (B) flex moduli, (C) tensile strength, (D) flexural strength, and (E) compressive strength data [Perusich, 1990]. 


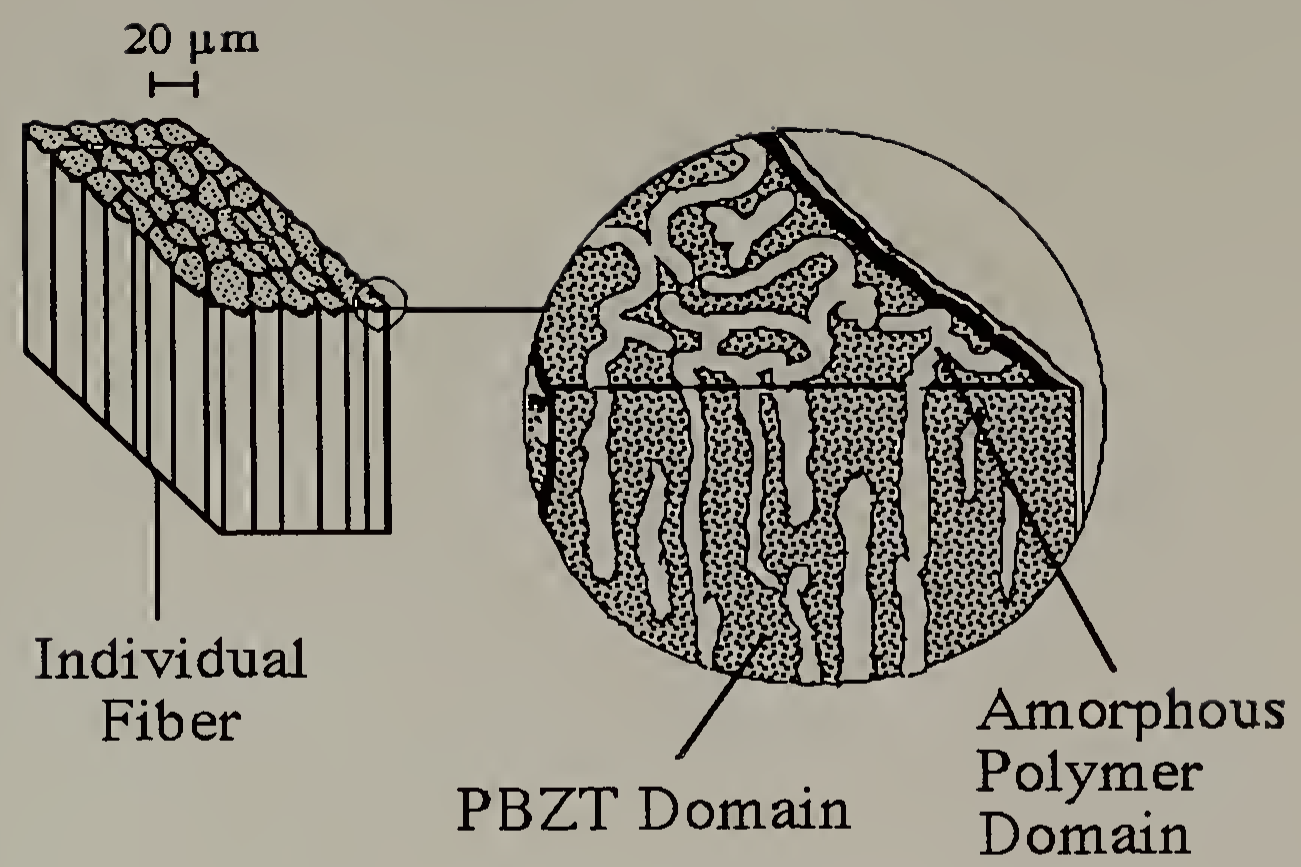

Figure 3.29 A schematic composite fabricated from an immiscible PBZT/coil polymer blend fiber. 
Bassett, B. R. and Yee, A. F., Polymer Composites, 11, pp. 10-18(1990). "A Method of Forming Composite Structures Using In Situ- Formed Liquid Crystal Polymer Fibers in a Thermoplastic Matrix".

Blundell, D. J.; Crick, R. A.; Fife, B.; Peacock, J.; Keller, A.; Waddon, A.; Journal of Materials Science, 24,2057-2064(1989). "Spherulitic Morphology of the Matrix of Thermoplastic PEEK/Carbon Fibre Aromatic Polymer Composites".

Cohen, Y. and Thomas, E. L., Macromolecules, 21, 433, (1988) "Microfibrillar Network of a Rigid Rod Polymer. 1. Visualization by Electron Microscopy".

Dawson, P. C., Blundell, D. J., Polymer, 21, 578, (1980). "X-ray data for poly(arylether ketones).

Farris, R. J.; Malone, M. F.; Schaffer, K. R.; "Morphological Studies of PBZT/PEKK Composite Fibers," October 1989 Report to E. I. du Pont de Nemours, Inc.

Flory, P. J., Macromolecules, 11, pp. 1138-1141(1978). "Statistical Thermodynamics of Mixtures of Rodlike Particles. 5. Mixtures with Random Coils".

Gabriel, C. A., PhD. Dissertation, Polymer Science and Engineering Department, University of Massachusetts (1987). "Processing and Properties of Composite Fibers of Poly (p-phenylene Benzobisthiazole) and Poly(ether ether ketone) or Nylon 6,6".

Hwang, C. Robin, PhD. Dissertation, Polymer Science and Engineering Department, University of Massachusetts (1989). "Molecular' and 'In-situ Network' Composites of Poly(p-Phenylene benzobisthiazole) and Nylons".

Hwang, C. R., Malone, M. F. and Farris, R. J., Journal of Materials Science, 26, pp. 1762-1768(1991). "Molecular composites of poly(p-phenylene benzobisthizole) with thermoplastics: coagulation studies".

Hwang, C. R., Malone, M. F., Farris, R. J., Martin, D. C. and Thomas, E. L., Journal of Materials Science 26, pp. 1768-1772(1991). "Microstructure and mechanical properties of in-situ network composite fibres of PBZT with nylon".

Hwang, W-F.; Wiff, D. R.; Verschoore, C., Price, G. E.; Helminiak, T. E.: Adams, W. W.; Polymer Engineering and Science, 23, pp. 784-788 (1983). "Solution Processing and Properties of Molecular Compostie Fibers and Films".

Krause S. J., Haddock, T. B.,Vezie,D. L., Lengert, P. G.,Hwang, W. F., Price, G E., Helminiak, T. E. , O'Brien, J F., Adams, W. W., Polymer, 29 (1988).

"Morphology and properties of rigid-rod poly(p-phenylene benzobisoxazole) (PBO) and stiff-chain poly $(2,5(6)$-benzoxazole)(ABPBO) fibers". 
Krause S. J., Haddock, T. , Price, G. E., Lengert, P. G., O'Brien, J. F., Helminiak, Thaddeus E. , Adams, W. W., Journal of Polymer Science: Part B: Polymer Physics, 24,1991,(1986). "Morphology of a Phase-Separated and a Molecular Composite PBT/ABPBI Polymer Blend".

Kumar, Satish and Wang, C. S., Polymer Communications, 29, pp. 355-356(1988). "Xray Study of PBT/nylon 66 Composite".

Lovinger, Andrew J. and Davis, D. D., Macromolecules, 19, pp. 1861-1867(1986).

"Solution Crystallization of Poly(ether ether ketone)".

Magliochetti, M., PhD. Dissertation, Polymer Science and Engineering Department, University of Massachusetts (1990). "Solution Viscosity and Phase Behavior of Lyotropic Liquid Crystalline and Flexible Polymers".

Minter, J. R., Structural Investigation of Fibers and Films of Polycpphenylenebenzobisthiazole), AFWAL-TR-82-4097, Vol I, Materials Laboratory (AFWAK/MLBP), May 1982.

Pottick, L. A., PhD. Dissertation, Polymer Science and Engineering Department, University of Massachusetts (1986). "The Influence of Drying on the Structure and Mechanics of Poly(p-Phenylene Benzobisthiazole) Fibers".

Rueda, D. R.; Ania, F.; Richarson A.; Ward I. M.; Balta, Calleja, F. J.; Polymer Communications, 24 pp. 258(1983), "X-ray Diffraction Study of Die Drawn Poly(aryletherketone) (PEEK)".

Perusich, Renee, unpublished data presented February 14, 1990 for U.S. Air Force.

Takayanagi Motowo, Interrelations Between Processing Structure and Properties of Polymeric Materials, pp. 43-56, J. C. Seferis and P. S. Theocaris, editors, Elsevier Science Publishers B. V. Amsterdam, The Netherlands (1984), "Polymer Composites of Rigid and Flexible Molecules".

Wycliffe, S. M., Malone, M.F. and Farris, R.J., Journal of Applied Polymer Science, 35, pp. 1955-1962(1987). "Processing and Properties of Composite Fibers of Poly (p-phenylene Benzobisthiazole)/ Nylon Fibers". 


\section{CHAPTER 4}

\section{LCP FIBER BLENDS BY MELT SPINNING}

\subsection{Introduction}

Polymeric materials are being investigated as structural components due to favorable specific strength and moduli and the range of other properties attained from a variety of intrinsic chemical structures. The chemical structure and the molecular weight ultimately determine the thermal, rheological, and solution properties and subsequently the practical processes of fabrication. Polymer blends are important due to the possibility of integrating favorable properties of several materials or introducing novel processes of fabrication.

Blends of liquid crystalline polymers (LCPs) and flexible coil or isotropic polymers can be fabricated by a variety of means; extruding melts or solutions constitutes one avenue [Krause et al., 1986, Hwang, 1989]. There are processing advantages with $\mathrm{LCP} /$ isotropic polymer melts because the addition of even small amounts of LCP can significantly lower the viscosity of the blend [Beery et al., 1991a].

If the LCP in the blend has a melting and decomposition temperature significantly higher than the melt temperature of the isotropic matrix, extruded blends quenched during flow will have liquid crystal fibrils formed in-situ that allow melt consolidation with heat and pressure [Bassett and Yee, 1990]. The final morphology will depend upon the morphology of the initial spin dope or melt, the rheology and the geometry of the processing flow, and, ultimately, the conditions of coagulation or solidification.

Blends of LCPs with poly(butylene terephthalate) (PBT) are of particular interest as self-reinforcing injection molding resins [Ajji et al., 1992, Beery et al, 1991(1,2), 
Zaldua et al., 1991]. The characterization of the morphology of immiscible polymers processed using various flow fields has been of interest [Delimoy et al., 1988]. This chapter discusses the domain morphology of a new thermotropic LCP blended with PBT during the processes of fiber spinning and drawing. The use of fibers to evaluate new LCPs is advantageous because of the number of samples that can be obtained from a small amount material recovered from chemical synthesis. The properties of fibers prior to drawing should give an indication of the properties of injection molded pieces.

The ease of orientation of the LCP during fabrication allows the attainment in undrawn fibers of the same strengths and moduli in drawn fibers. This effect can be advantageous if other properties such as thermal expansion and shrinkage are considered.

The characterization of the morphology of immiscible polymers processed using various flow fields has been of interest [Delimoy et al., 1988, Beery et al, 1991a, 1991b, Zaldua et al., 1991]. This chapter discusses the domain morphology of a new thermotropic LCP blended with PBT during the processes of fiber spinning and drawing. The use of fibers to evaluate new LCPs is advantageous because of the number of samples that can be obtained from a relatively small amount material. The properties of fibers prior to drawing may give an indication of the properties of injection molded pieces.

\section{$4.2 \quad$ Experimental}

\subsubsection{Materials}

The liquid crystal polymer (LCP) studied consists of a triad (TR) of carbonyllinked phenyl rings with a random distribution of four and six $(4,6)$ aliphatic flexible spacers as shown in Figure 4.1. There is a one to one ratio of the four to the six carbon flexible spacers. Prof. Kantor's group at the University of Massachusetts at Amherst 
supplied the TR4,6 polymer. The TR4,6 LCP was solution blended with poly(butylene terephthalate) which had weight average molecular weight $(\mathrm{Mw})$ of 33,000 . Weighed portions of polymers were dissolved in triflouroacetic acid at concentrations less than $5 \%$ $\mathrm{w} / \mathrm{w}$. The polymer mixture precipitated when the solution was dripped into acetone to form a powder or beads. After neutralizing by washing the precipitate twice with a 5\% sodium bicarbonate solution, it was washed with distilled water twice. The material was then washed in acetone and dried at $75^{\circ} \mathrm{C}$ for at least twelve hours in a vacuum oven.

\subsubsection{Thermal analysis}

Differential Scanning Calorimetry (DSC) was performed on a Dupont 2000 under nitrogen. Approximately 5 milligram samples were heated to above $300^{\circ} \mathrm{C}$ and quenched to $20^{\circ} \mathrm{C}$ at $10^{\circ} \mathrm{C} / \mathrm{min}$. before obtaining the data at a heating rate of $10^{\circ} \mathrm{C} / \mathrm{min}$.

\subsubsection{Fiber spinning and properties}

The TR4,6/PBT blends were melted and pressed into sheets at $230^{\circ} \mathrm{C}$. A plug was molded by pressing at $205^{\circ} \mathrm{C}$. Fibers were spun at $245^{\circ} \mathrm{C}$ on a capillary rheometer using a $40 \mathrm{~L} / \mathrm{D} 0.001$ inch diameter die with a take up device using a variable speed motor to control the stretch ratio. The stretch ratio is the linear take-up rate of the fiber divided by the linear throughput of material through the spinning die. The stretch ratio values were varied from 1 to 110 in these experiments. Figure 4.2 shows a schematic of the fiber spinning process and the subsequent hot drawing given to some of the fibers. 
A

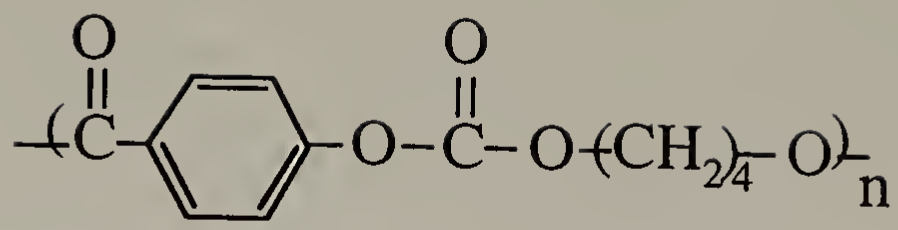

B<smiles>CCOc1ccc(C(=O)Oc2ccc(C(=O)Oc3ccc(C(C)=O)cc3)cc2)cc1</smiles>

Figure 4.1 Structures of (A) poly(butylene terephthalate) and (B) the random TR4,6 (1/1) liquid crystal polymer used for blending.

Table 4.1 Thermal Properties of PBT and TR 4,6

$$
\mathrm{T}_{\mathrm{m}}\left({ }^{\circ} \mathrm{C}\right) \quad \quad \mathrm{T}_{\mathrm{decomp}}\left({ }^{\circ} \mathrm{C}\right) \quad \eta(\mathrm{dl} / \mathrm{g})
$$

PBT

225

TR $-4,6$

Most of the orientation developed during the processing of the liquid crystal polymers is due to the elongational flow arising at the die due to the stretch operation. Elongational flow fields orient polymeric liquid crystalline melts to a greater extent relative to shear flow fields [Kenig, 1987 and Kenig, 1989].

After spinning, the fibers were hot drawn by rewinding the fiber from one bobbin to another using two variable speed motors set at different speeds. Hot draw ratio values were typically between 3.5 and 5 . The fiber passed over a hot plate set at a specified 
temperature in the draw zone as shown in the Figure 4.2B. Tensile specimens were fixed on tabs of set lengths according to the procedure of ASTM D3379-75. The fiber diameters were determined with a Zeiss microscope. The use of tabs to hold the fibers minimizes damage to the specimens before testing [De Teresa, Porter, and Farris, 1984].

A

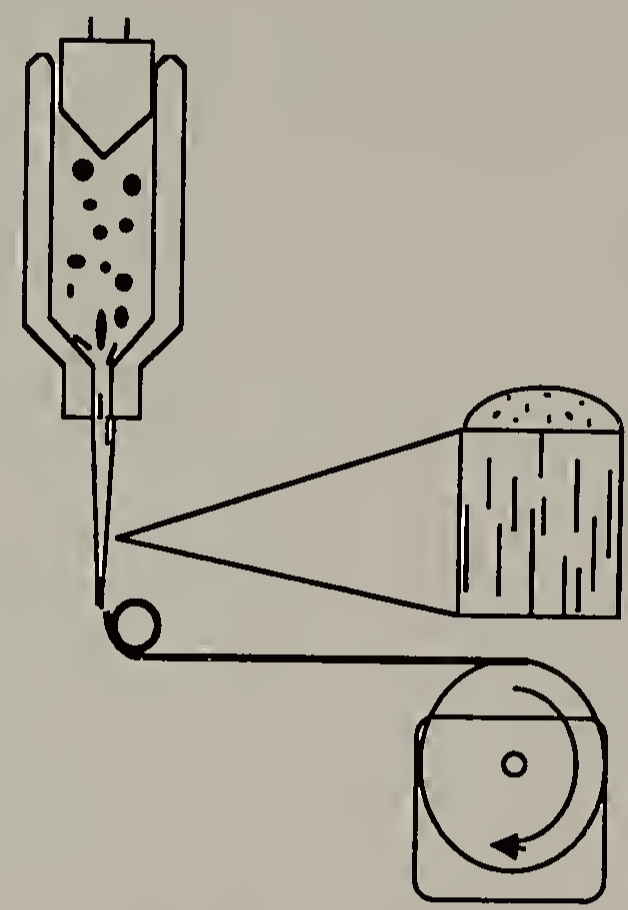

B

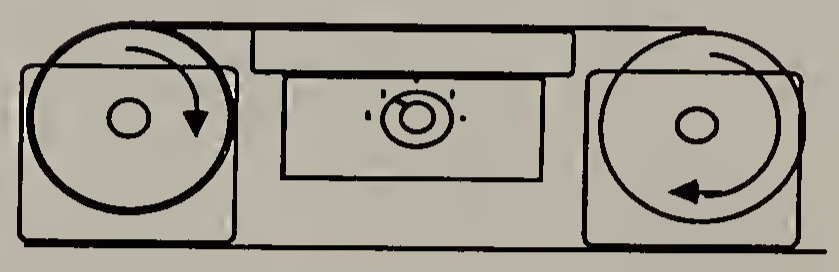

Figure 4.2 Schematics of spinning fiber blend (A) and post hot draw (B). 


\subsubsection{Microscopy}

A JEOL $100 \mathrm{CX}$ operated in SEM mode and a JEOL $35 \mathrm{FX}$ operated at $20 \mathrm{kV}$ provided SEM images of the low temperature fractured surfaces of the fibers. The fibers were placed in liquid nitrogen and fractured by bending after scoring with a razor blade. A gold coating on the specimens reduced charge build-up and the subsequent discharging to the detector. Polaroid P/N film was used to record the SEM images.

Pelco Medcast Quik-Mix Kit epoxy of medium hardness was employed as the embedding medium for the fiber specimens. Mounting the films in a silicon rubber or polyethylene mold facilitates microtomy for cross and longitudinal sections. The epoxy is firm enough to microtome after curing at $60^{\circ} \mathrm{C}$ for 16 to 20 hours. The embedded film samples are trimmed to approximately 1 square millimeter in size and sectioned a Sorvall MT2-B ultra-microtome. A diamond knife was used to microtome the LCP/PBT fibers at ambient temperatures using water to float the sections after cutting. For TEM examination, copper grids supported the thin sections retrieved from the water surface.

Thicker sections were viewed on a Zeiss polarizing microscope. TEM images of the film thin sections were obtained using a JEOL 100CX TEM or a JEOL 2000FX TEM at $100 \mathrm{kV}$ using Kodak SO-163 electron image film.

\subsection{Results and Discussion}

\subsubsection{Crystallization}

Depending upon the experimental conditions, PBT crystallizes in two different spherulitic forms; one being normal and the other being abnormal as defined by the type of laser light $\mathrm{H}_{V}$ scattering patterns exhibited [Misra and Stein, 1980]. The two optical textures are not due to different unit cell structures, but are the result of differing orientations of the specific crystalline planes with respect to the spherulite radius direction. The data indicates that the lamella ribbons in the abnormal spherulites are 
shorter; allowing inclined packing of lamella with respect to the spherulite radius direction [Roche, Stein, and Thomas, 1980].

Spherulites with mixed normal and abnormal optical textures have been observed for both the solution cast and the cooled PBT samples [Roche, Stein, and Thomas, 1980, Ludwig and Eyerer, 1988]. The abnormal spherulites form at rapid cooling rates from the melt. The observed melting point of crystals in the normal spherulites $\left(220^{\circ} \mathrm{C}\right)$ is lower than in the abnormal ones $\left(25^{\circ} \mathrm{C}\right)$ [Ludwig and Eyerer, 1988]. Annealing experiments show the lower temperature peak is due to the melting of crystals that form upon annealing [Runt and Yeh, 1989].

Increasing the content of TR4,6 polymer suppresses the PBT melting peak at $221^{\circ} \mathrm{C}$ and shifts the higher temperature peak at $225^{\circ} \mathrm{C}$ as Figure 4.4 demonstrates. This indicates that the presence of the LCP disrupts the formation of more perfect crystals in blends compared with pure PBT at the same conditions. From the similarity of their chemical structure, one would expect a some melt miscibility of the polymers if they were in the isotropic melt. However, any limited miscibility would not be mutual because the orientational order of the LCP phase has been theoretically shown to exclude the isotropic coils due to entropy considerations [Koningsveld, 1989].

Table 4.2 shows the effects of LCP content upon the crystalline melting temperature of the PBT. The calculated LCP mole fraction was obtained from an expression given by Flory [Flory, 1956],

$$
\frac{1}{T_{m}}+\frac{1}{T_{m}{ }^{0}}=-\frac{R}{\Delta H_{P B T}} \ln N_{P B T}
$$

where $\mathrm{Tm}$ is the observed melting temperature, $\operatorname{Tm}^{\circ}$ is the melting temperature of the pure polymer, $\mathrm{R}$ is the gas constant, $\triangle \mathrm{H}_{\mathrm{PBT}}$ is the heat of fusion, and $\mathrm{N}_{\mathrm{PBT}}$ the mole fraction of the PBT. An estimate of the mole fraction of LCP, (1-NPBT), is based upon number of monomer units and not the number average degree of polymerization. The 
values in the last column in Table 2.2 indicate that some melting point depression due to LCP in the PBT crystal could be occurring. The PBT crystals appear to contain at least 2 percent LCP. This miscibility may be due to transesterfication. The data suggest that kinetics of PBT crystallization and the formation of copolymer by transesterfication does not allow the exclusion of the LCP from the PBT crystal.

The DSC scans in Figure 4.5 show that the stability of the melt blend for various times at the processing temperature. Annealing above the melt temperature does not affect the amount of crystallization of the PBT upon cooling. Any transesterfication that may be occurring during annealing is not evident in the subsequent crystallization behavior of the PBT phase. The relative effect of the TR-4 LCP upon the PBT melting peak at $220^{\circ} \mathrm{C}$ is not changed with annealing at $250^{\circ} \mathrm{C}$ which is the processing temperature used during the fabrication of fibers.

Figure 4.3 shows a DSC scan of the TR4,6 random copolymer showing a crystal to nematic transition at $220^{\circ} \mathrm{C}$. The relative amount of crystallinity in TR4,6 is much less than observed in pure PBT as Figure 4.4A attests showing the DSC scans of TR4,6/PBT blend specimens with varying LCP content.

Table 4.2 Effect of LCP Content and Annealing

$\begin{array}{ccccc}\begin{array}{c}\text { \% TR4,6 } \\ \text { in Blend }\end{array} & \begin{array}{c}\mathrm{Tm}\left({ }^{\circ} \mathrm{C}\right) \\ \text { after } 0 \mathrm{~min}\end{array} & \begin{array}{c}\mathrm{Tm}\left({ }^{\circ} \mathrm{C}\right) \\ \text { after } 5 \mathrm{~min}\end{array} & \begin{array}{c}\mathrm{Tm}\left({ }^{\circ} \mathrm{C}\right) \\ \text { after } 15 \mathrm{~min}\end{array} & \begin{array}{c}\text { Mole Fraction } \\ \text { LCP in PBT } \\ \text { phase* }\end{array} \\ 0 & 225.4 & 225.3 & 224.7 & 0 \\ 5 & 223.1 & 223.0 & 223.4 & 0.02 \\ 10 & 221.8 & 222.7 & 223.1 & 0.02 \\ 20 & 221.8 & 221.3 & 222.4 & 0.03\end{array}$




\subsubsection{Tensile properties}

Figure 4.6 shows the effect of the spin stretch ratio during fiber fabrication upon the tensile modulus and the breaking strength of fibers with various TR4,6 content. No fibers were spun from the pure TR4,6 polymer due to the limited quantity of material. At very low stretch ratios, the addition of any LCP results in a significant decrease in the breaking strength. The reduction in strength may be due defects generated during processing or low orientation in the liquid crystalline domains making them act as defects. At moderate stretch ratios the modulus and strength values of the blends are comparable to the PBT matrix indicating a lack of reinforcement. Assuming similar orientations and crystallinity, the moduli and strengths of the LCP and that of PBT are probably comparable given their similarity in chemical structure. The advantage of the LCP is the ease at which it orients in the elongational flow during the fiber spinning process. The neat PBT needs an additional hot draw to attain the same modulus value as as-spun 80/20 PBT/LCP. The question remains whether the increase observed in the asspun fiber is due to reinforcement or due to the induced orientation in the PBT by the LCP.

Only the 20/80 TR4,6/PBT blend fiber (at a stretch ratio of 109) shows improved tensile properties over the undrawn PBT fiber. This result suggests there may be a criterion of domain size for reinforcement to occur in undrawn as-spun fibers. The concentration at which strengthening occurs would be important for applications in which post drawing is not pertinent such as injection molding.

If the elongational flow during processing was sufficient to fully orient the LCP, one would expect post drawing to have a limited effect upon tensile properties as Figure 4.7 illustrates. After the PBT matrix is oriented by hot drawing, the relative effect of the LCP upon modulus and strength values diminishes to insignificance even in the 20/80 TR4,6/PBT blend. The strength of the oriented PBT dominates the properties of the hot 
drawn fibers; this shows the modulus of the LCP may not offer much improvement to this matrix.

However, the ease of orientation of the LCP during fabrication allows the attainment in undrawn fibers of the same strengths and moduli in drawn fibers. This effect can be advantageous if other properties such as thermal expansion and shrinkage are considered.

\subsubsection{Domain Orientation}

When the TR4,6/PBT blend is above the melt temperatures of its components, the LCP can be easily distinguished from the PBT matrix by viewing between crossed polarizers in an optical microscope. However, one must properly orient the sample relative to the polarizers to take advantage of the birefringence of the LCP; furthermore, it is beneficial if the LCP is globally oriented.

Figure 4.8 shows optical micrographs of the TR4,6/PBT blends of various compositions pressed between glass microscope slides and viewed between crossed polarizers while on a hot stage at $250^{\circ} \mathrm{C}$. The solution blending procedure appears to well disperse the TR4,6 into PBT matrix. The TR4,6 domain sizes in the $5 \%$ and $10 \%$ TR4,6 blends are about a micron in size. In the $20 \%$ blend, the TR4,6 domains are easily sheared between the microscope slide and cover slip to fibrillar structures as Figure 4.8D shows.

To view the LCP domain structure in the as-spun and hot drawn fibers, longitudinal thin sections of the fibers were placed on microscope slides. The fibers were fabricated with a spin stretch ratio of 95 and hot drawn (D.R. $=5$ ) to orient the PBT. The microtoming caused some deformation of the section obscuring any details of interest under crossed polarizers (see Figure 4.9). Longitudinal sections of the as-spun and heat treated fibers were heated on a hot stage to $240^{\circ} \mathrm{C}$; the optical micrographs of 
the heat treated sections (with crossed polarizers) are shown in Figures $4.9 \mathrm{C}$ and $4.9 \mathrm{E}$, respectively. The widths of the fiber sections did not change after heating showing there was no significant deformation of the sections due to the thermal treatment. However, the LCP domain structure does become more obvious; there are only slightly extended domains in the as-spun fiber and highly elongated LCP domains in the hot drawn fiber.

When the hot drawn fiber is placed parallel to the direction of polarization it goes extinct, this indicates directionality of the liquid crystalline domains. The domains in the as-spun fiber do not go extinct when rotated between the cross polarizers indicating a lack of directionality in the birefringent LCP domains.

The LCP particles in the as-spun fibers can be observed in SEM images of low temperature fracture surfaces shown in Figure 4.10. The micrographs show a good dispersion of the LCP in a solid PBT matrix. The ease at which the particles are observed indicates interface between the respective polymers is easily fractured. However, one cannot conclude there is poor adhesion between the phases because the fracture may be occurring cohesively within the LCP or PBT.

The LCP particles are on the order of 0.1 to 1 micron in diameter with the lengths somewhat longer. Evidence for longer domains can also be observed in the optical micrograph in Figure 4.9. The elongated domains might not be considered fibrillar, but still appear to cause an increase in the modulus for the melt stretched fiber at the $20 \%$ LCP content as Figure 4.7A shows.

The LCP domains appear to deform with the PBT matrix during the hot draw at $166^{\circ} \mathrm{C}$. Figure 4.11 shows a freeze fracture of an 80/20 TR4,6/PBT fiber with a hot draw ratio of 5 (and at $166^{\circ} \mathrm{C}$ ). The size of the fibrils is the same as the birefringent domains observed in the polarized optical micrograph in Figure 4.9E. 


\subsubsection{Molecular Orientation}

To compare molecular orientation in fibers, electron diffraction patterns were obtained from the thin sections. Figures 4.12 show bright and dark field TEM images along with a diffraction pattern for a longitudinal thin section of PBT fiber spun and hot drawn at the same conditions as the blend fiber. The diagonal lines are due to knife marks during microtoming. There was no evidence of crystallites or fibril structure in the bright or dark field images.

In Figure $4.13 \mathrm{C}$, the reflections in the diffraction pattern are smeared in the knife direction which may be the result of some deformation. There is some debri evident in the section. All the dark field images were made using the equatorial reflections in the electron diffraction patterns.

Figure 4.13 shows similar images for longitudinal thin sections from as-spun $80 / 20$ TR4,6/ PBT fiber. There is a lack of orientation in the diffraction pattern. The reflections are very diffuse which may be an indication of very small crystallites, but it could also be a result of the thickness of the section.

The arrows point out regions in the dark field (Figure 4.13B) that are the same size as the LCP particles noted in the SEM images in the undrawn fiber in Figure 4-10. Higher crystallinity in the PBT phase (as the DSC data suggest) would result in the PBT regions to appear light and the TR4,6 to appear relatively darker. The TR4,6 domains appear to have aspect ratios as high as 10 , though most have much lower. The major parameters determining if elongated domains will form are the capillary number (the ratio of viscous to the surface forces) and the viscosity ratio (of the inclusion material to the matrix). Assuming the surface tension between PBT and TR4,6 to be very low, the viscosity ratio and the size of the droplet would determine the extent of LCP droplet deformation. The tensile modulus data in Figure 4.7A indicates that the elongated structures in the as-spun fiber do appear to be reinforcing. 
In Figure $4.13 \mathrm{C}$, the diffuse pattern results from the thickness of the section, but one gets an indication of the relative molecular orientation in the fiber. Due to the similarity of the chemical structures it is assumed the equatorial reflections observed for the blend specimens are due to both polymers.

After hot drawing, the orientation (in Figure 4.14) is comparable to that observed in the PBT drawn fiber.

The sections on copper grids were given the same heat treatment as the sections on glass slides; only the drawn 80/20 TR4,6/ PBT fiber section was intact after heating to $240^{\circ} \mathrm{C}$ and quenching, though there was some deformation. The same treatment on unoriented PBT and the as-spun 20\% TR4,6/PBT fibers resulted in complete melting of the fiber section so that observation was not possible. One would expect the relaxation of orientation of the PBT to be faster than the relaxation of the oriented TR4,6 polymer.

The electron diffraction pattern in Figure 4.13C still exhibits orientation as one would expect from the birefringence observed in the optical micrographs from the same sample. The TEM images show elongated structures similar in size ( $1 \mu \mathrm{m}$ in diameter) to the fibrils seen in the TEM.

\subsection{Conclusion}

During fiber spinning, the shear and elongational flow was sufficient for molecular orientation of the LCP but not for significant for fine fibril formation. The observation of the elongation of the LCP domains indicates the TR4,6 polymer can be hot drawn along with the PBT matrix in the fiber. The elongation of the LCP does not significantly increase the properties of the fiber over oriented PBT.

If the data indicates the processing conditions are sufficient to fully orient the TR4,6 LCP, the tensile properties of the TR4,6 polymer appear not to be significantly different from oriented PBT. 
The crystallization of the PBT is affected by the presence of the TR4,6 polymer by lowering the PBT melt temperature. The data indicates a disruption of the crystal structure due to a slight miscibility of the TR4,6 polymer in the PBT or formation of PBT / TR4,6 block copolymer by transesterfication. The kinectics of PBT crystallization may trap the LCP into the PBT crystallites.

The ease of orientation of the LCP during spinning suggests that the LCP would offer improvement for injection molded parts where post drawing treatments are not applicable. 


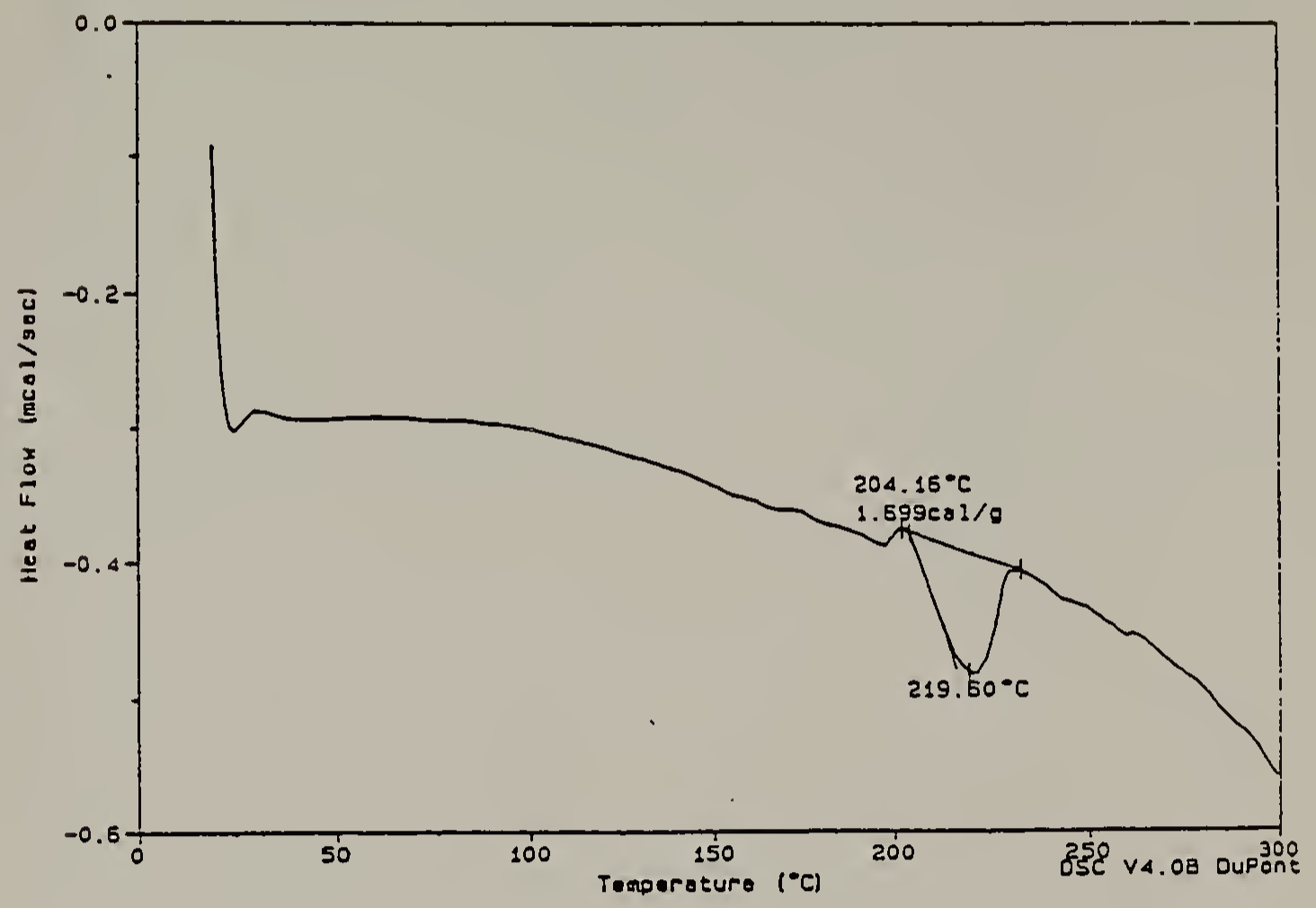

Figure 4.3 DSC scan of the TR4,6 random copolymer. 


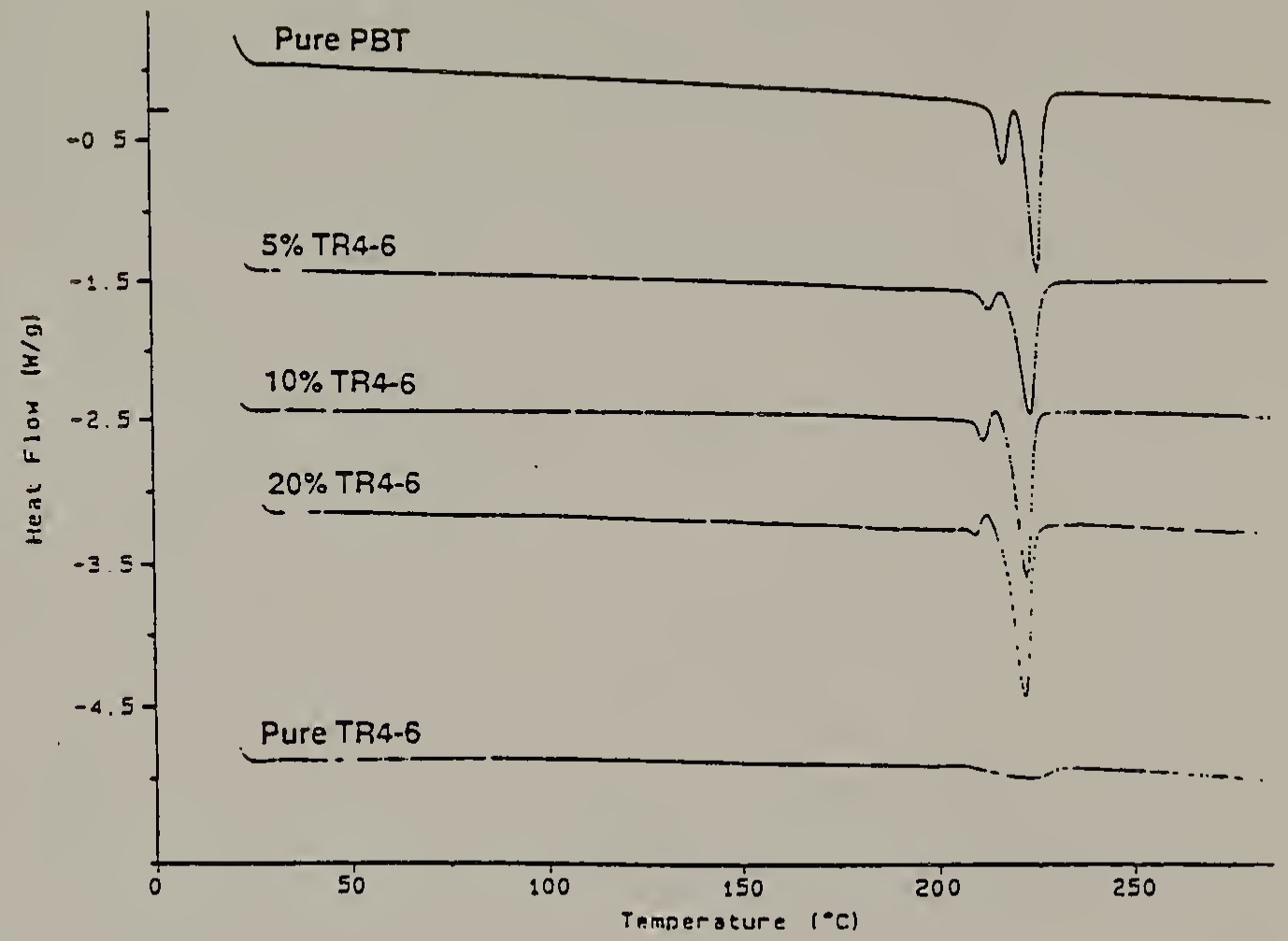

Figure 4.4 DSC scans of TR4,6/PBT blend specimens with varying LCP content. 


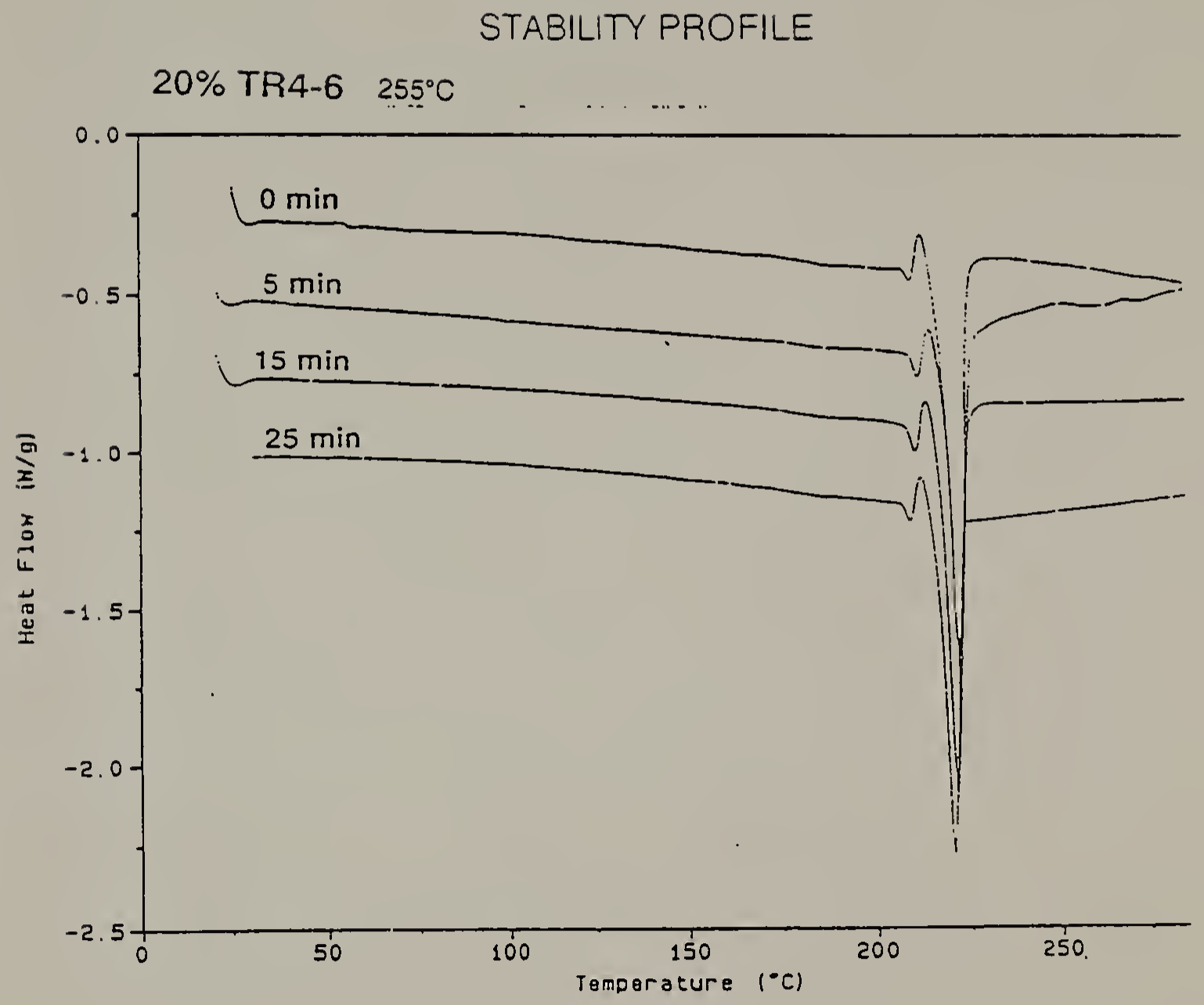

Figure 4.5 DSC scans of the $80 / 20 \mathrm{TR} 4,6 / \mathrm{PBT}$ after various times at $255^{\circ} \mathrm{C}$. 
A

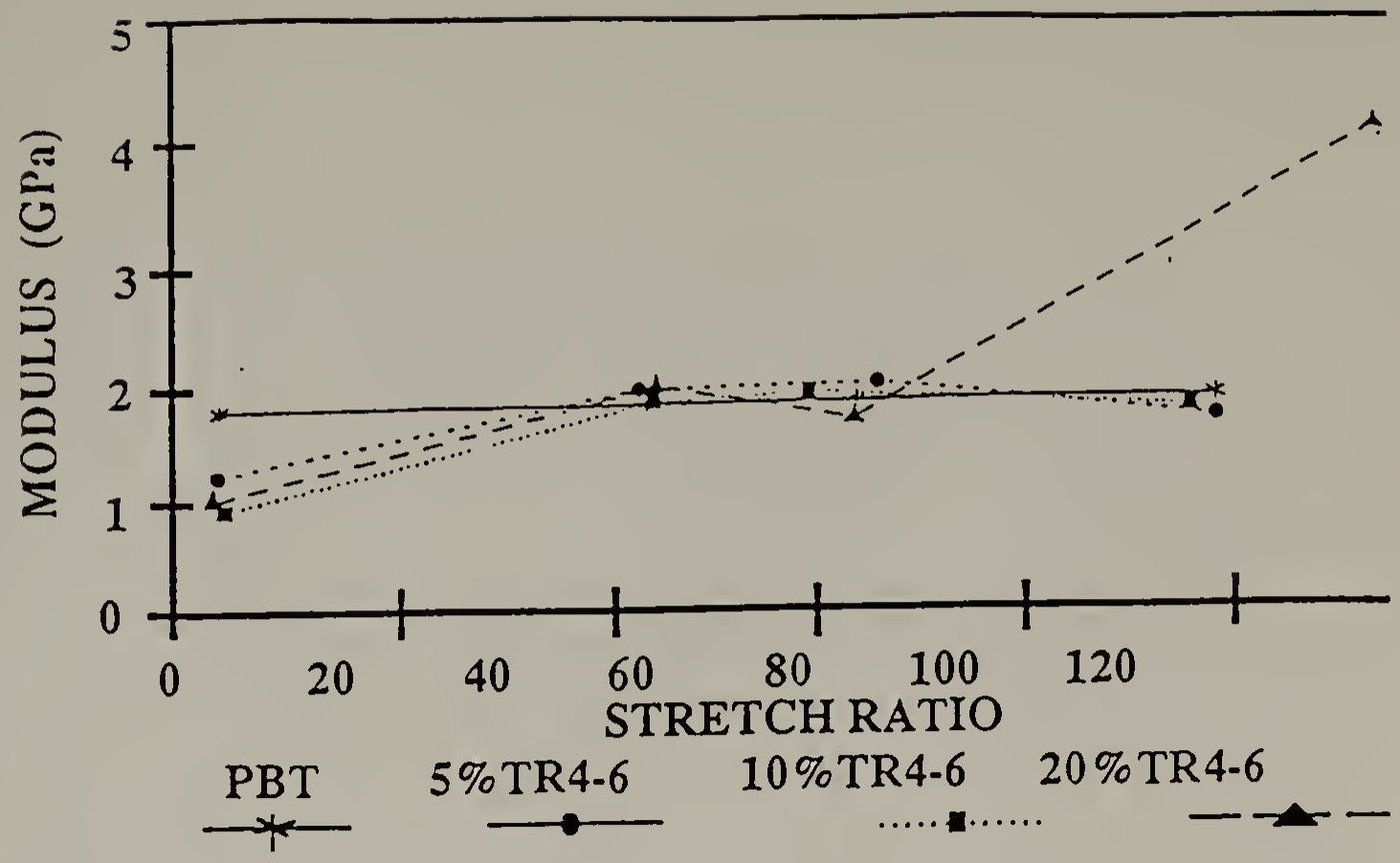

B

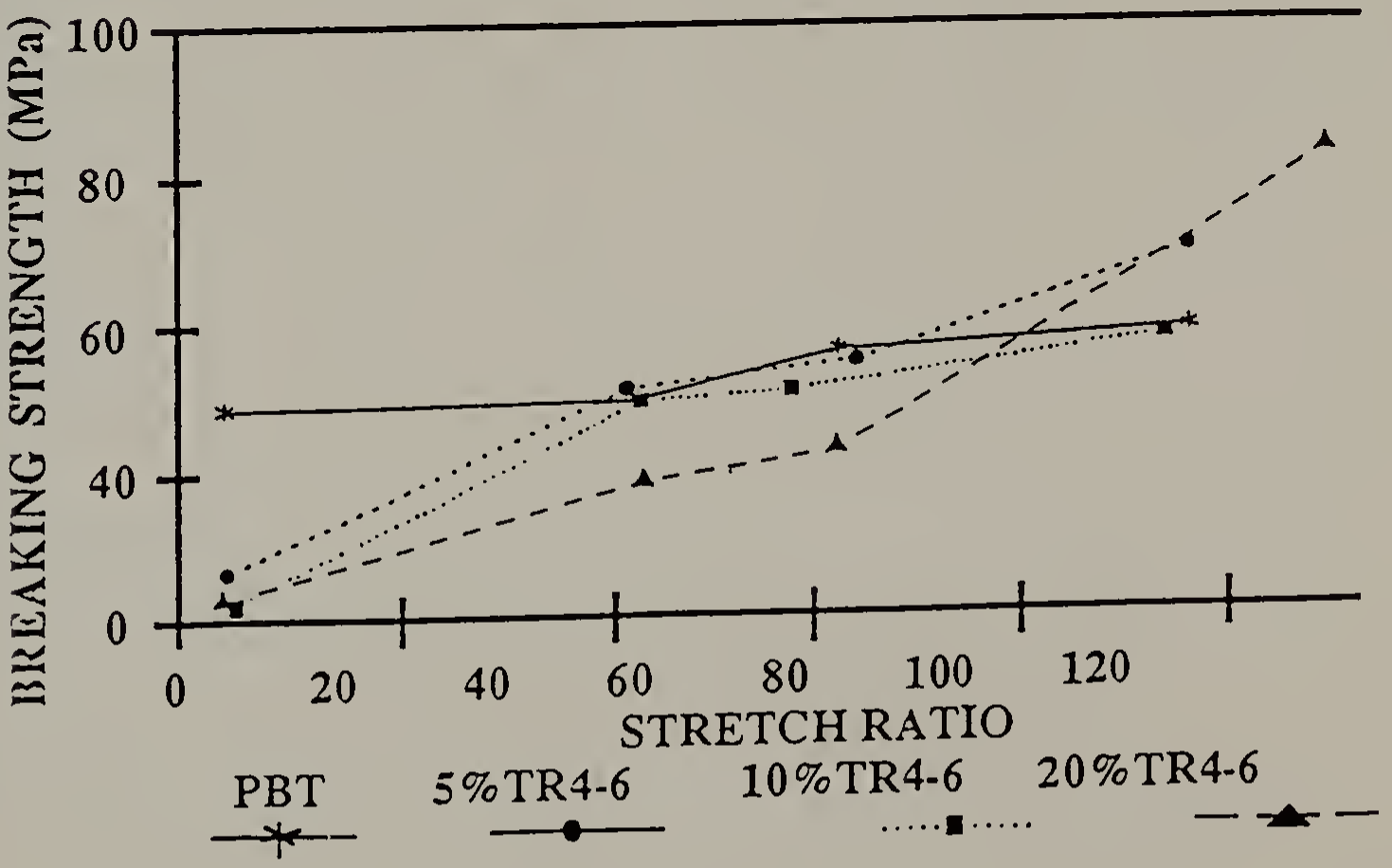

Figure 4.6 Graph showing the effect of spin stretch ratio upon (A) the modulus and (B) the breaking strength of as-spun fibers. 
A

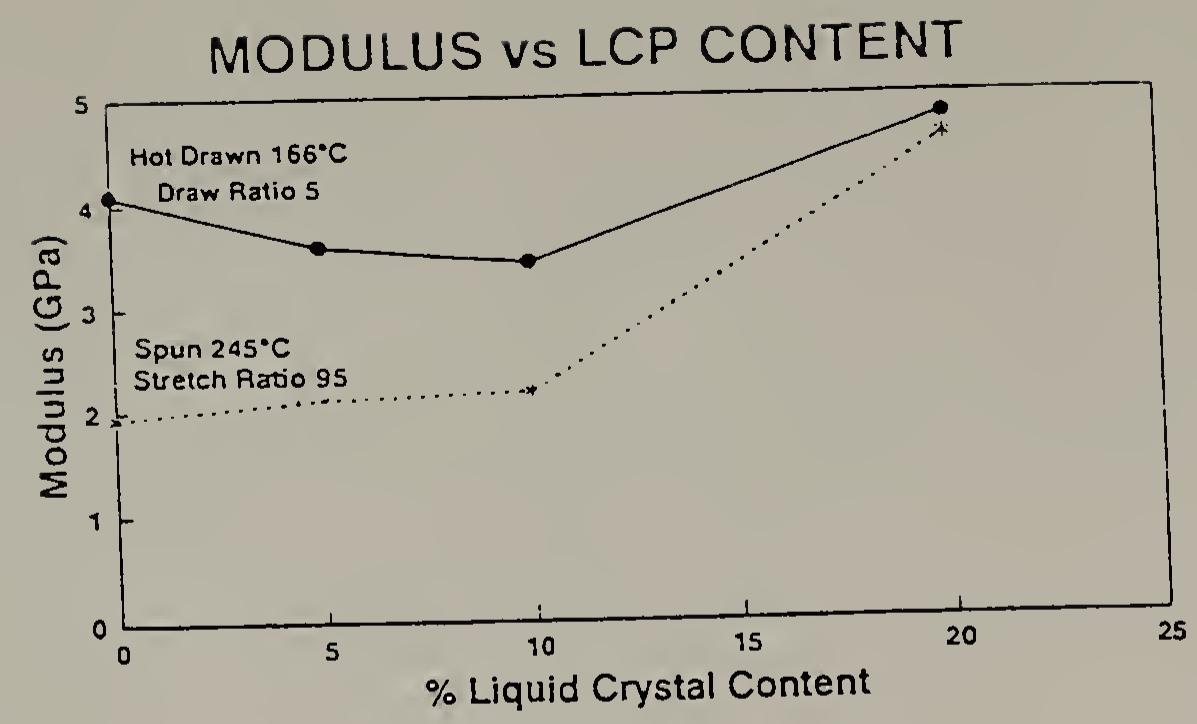

B

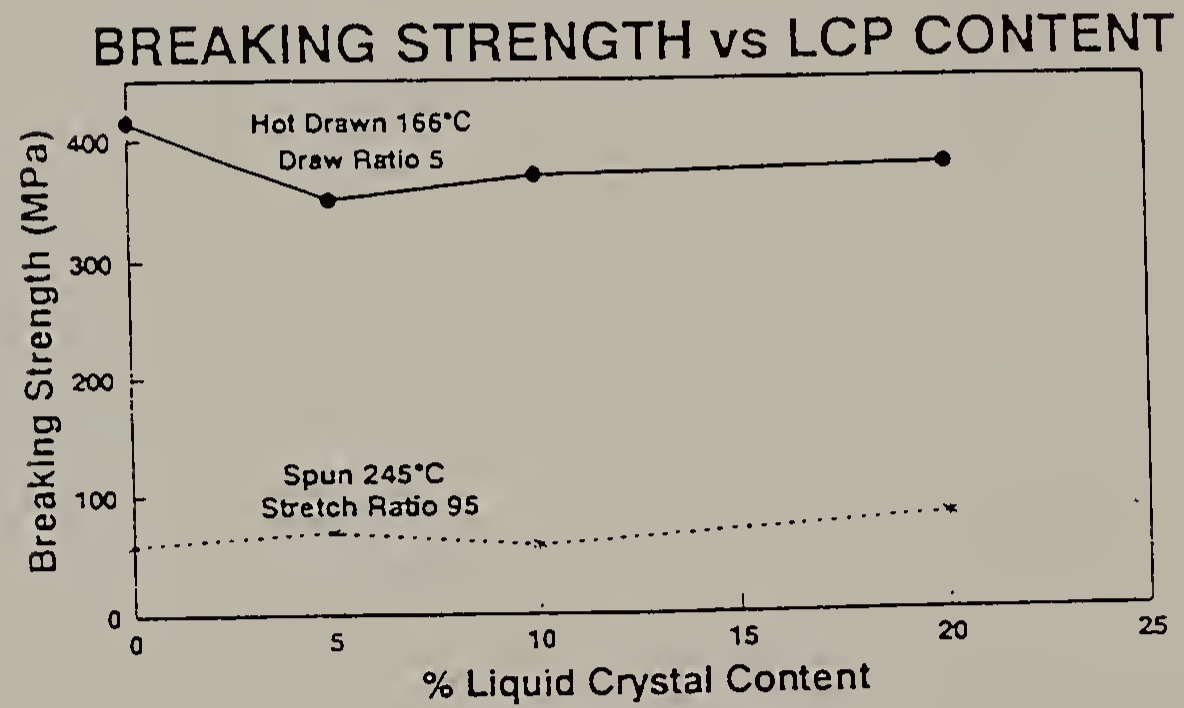

Figure 4.7 Graph showing the effect of liquid crystal content upon (A) the modulus and (B) the breaking strength of as-spun and post hot drawn fibers. 

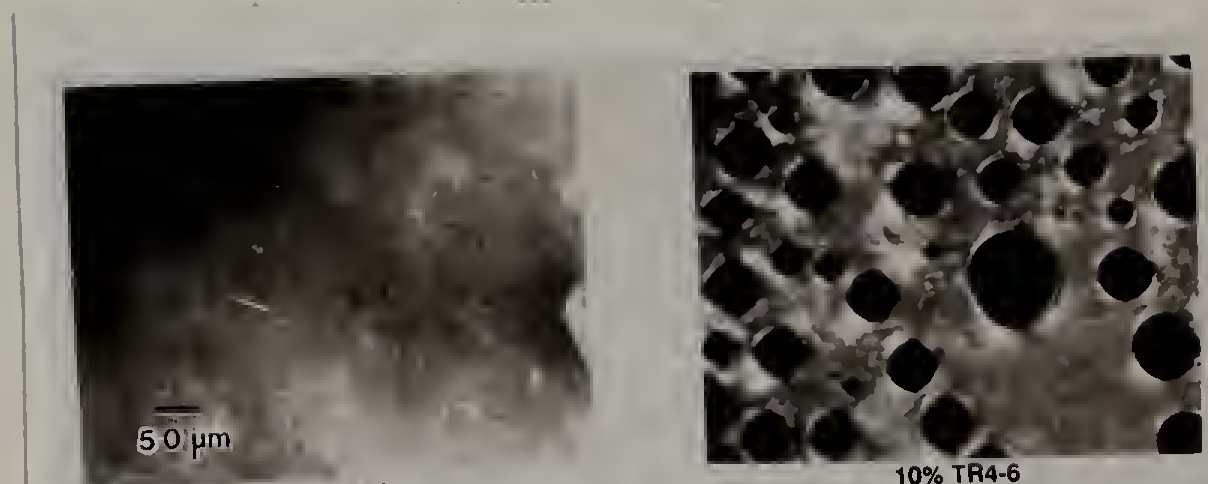

$5 \%$ TR4-6

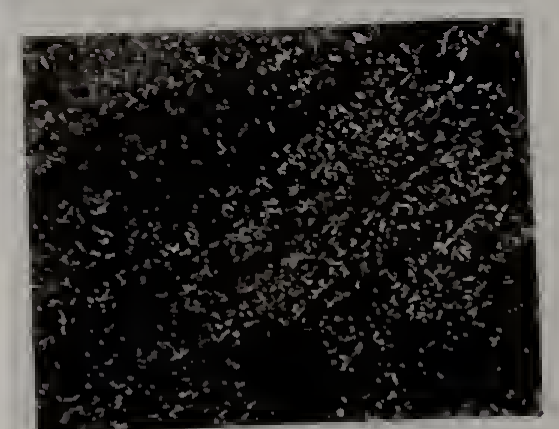

$20 \%$ TR4-6

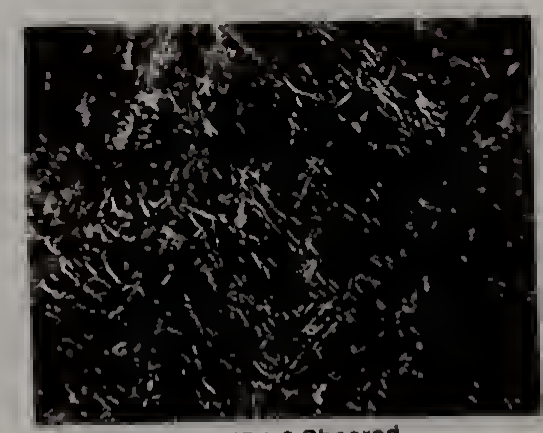

20\% TR4-6 Sheared

Figure 4.8 Optical micrographs of TR4,6/PBT blends with crossed polarizers parallel to the image edges. 


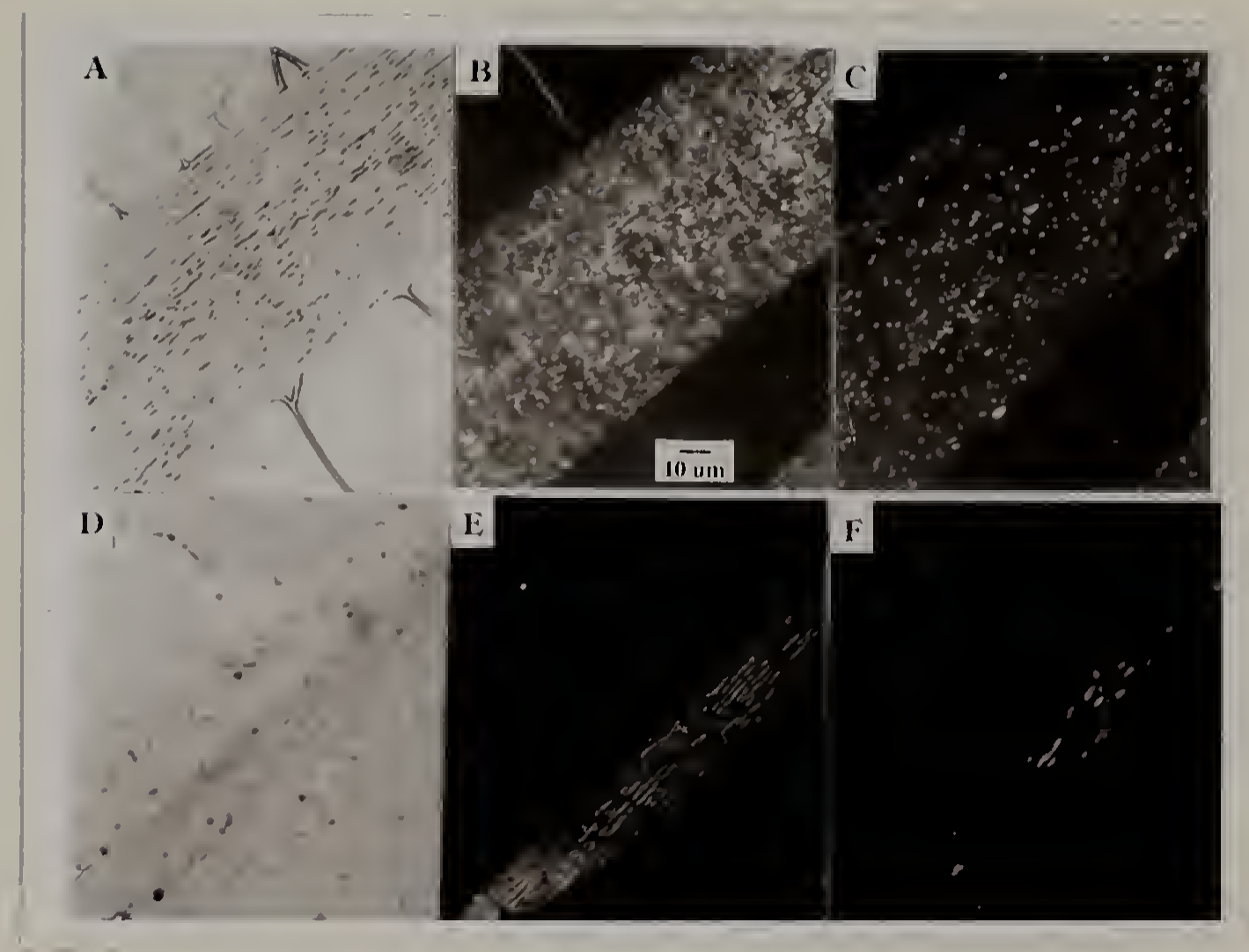

Figure 4.9 Thin section of the as-spun and post drawn 20/80 TR 4,6/PBT fiber. (A) as-spun, no polarizers, (B) post hot drawn, no polarizers, (C) as-spun, cross polarizers, (D) post hot drawn, cross polarizers, (E) as-spun, cross polarizers, $240^{\circ} \mathrm{C}(\mathrm{F})$ post hot drawn, cross polarizers, $240^{\circ} \mathrm{C}$. The polarizers are parallel to the image edges. 


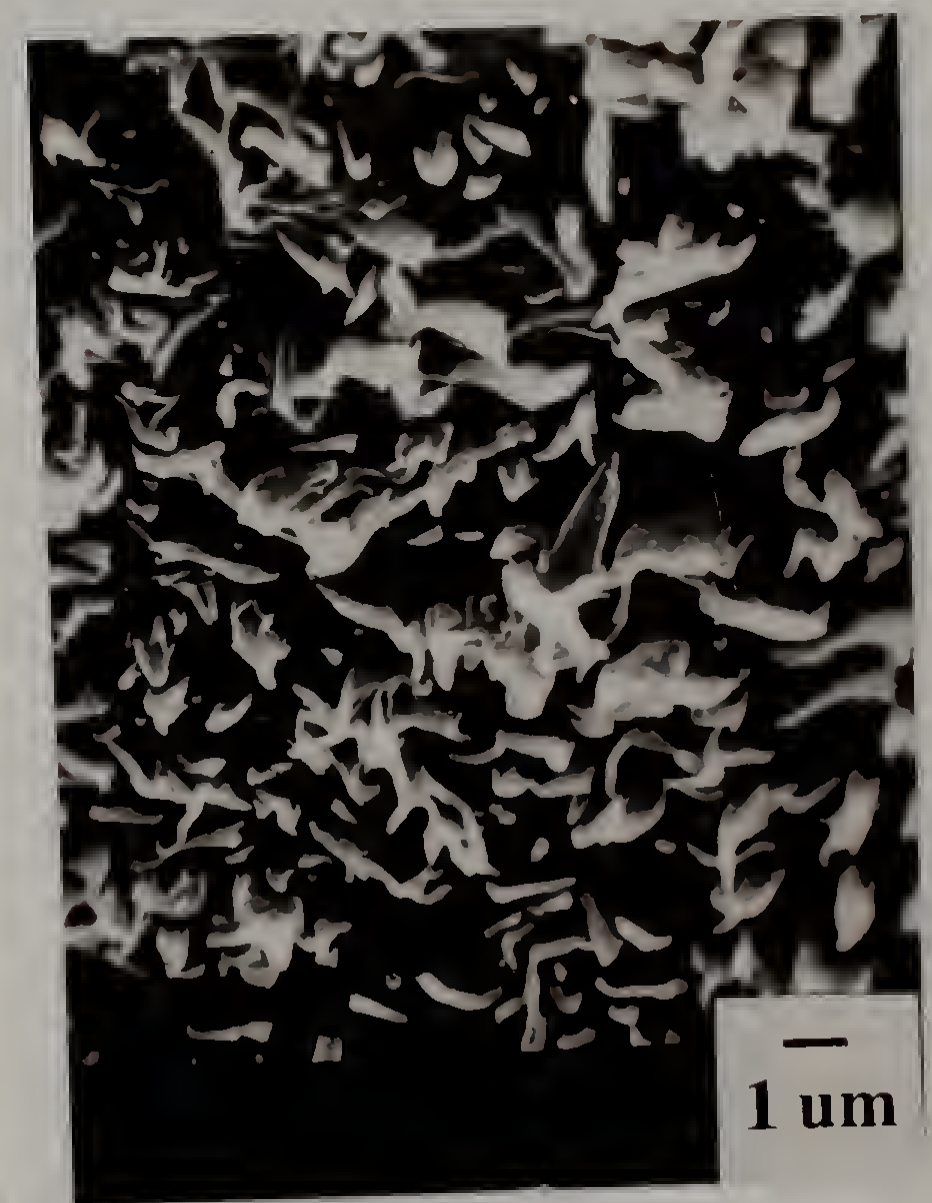

Figure 4.10

An SEM of a 20/80 TR 4,6/PBT as-spun fiber shows a transverse cryogenic fracture displaying the particulate nature of the LCP inclusions. 

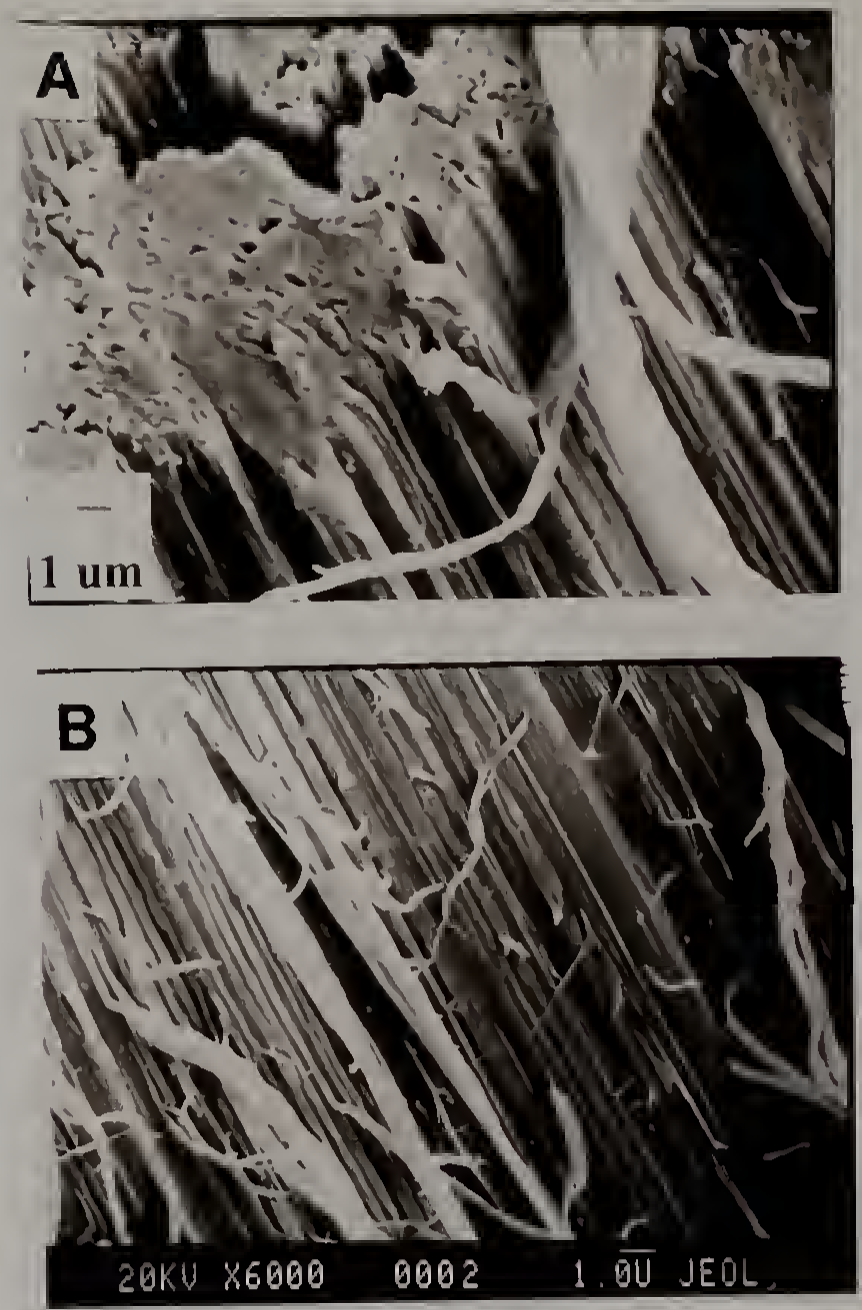

Figure 4.11 An SEM of a 20/80 TR 4,6/PBT hot drawn fiber showing an (A) transverse and (B) longitudinal cryogenic fracture displaying a fibrillar nature. 


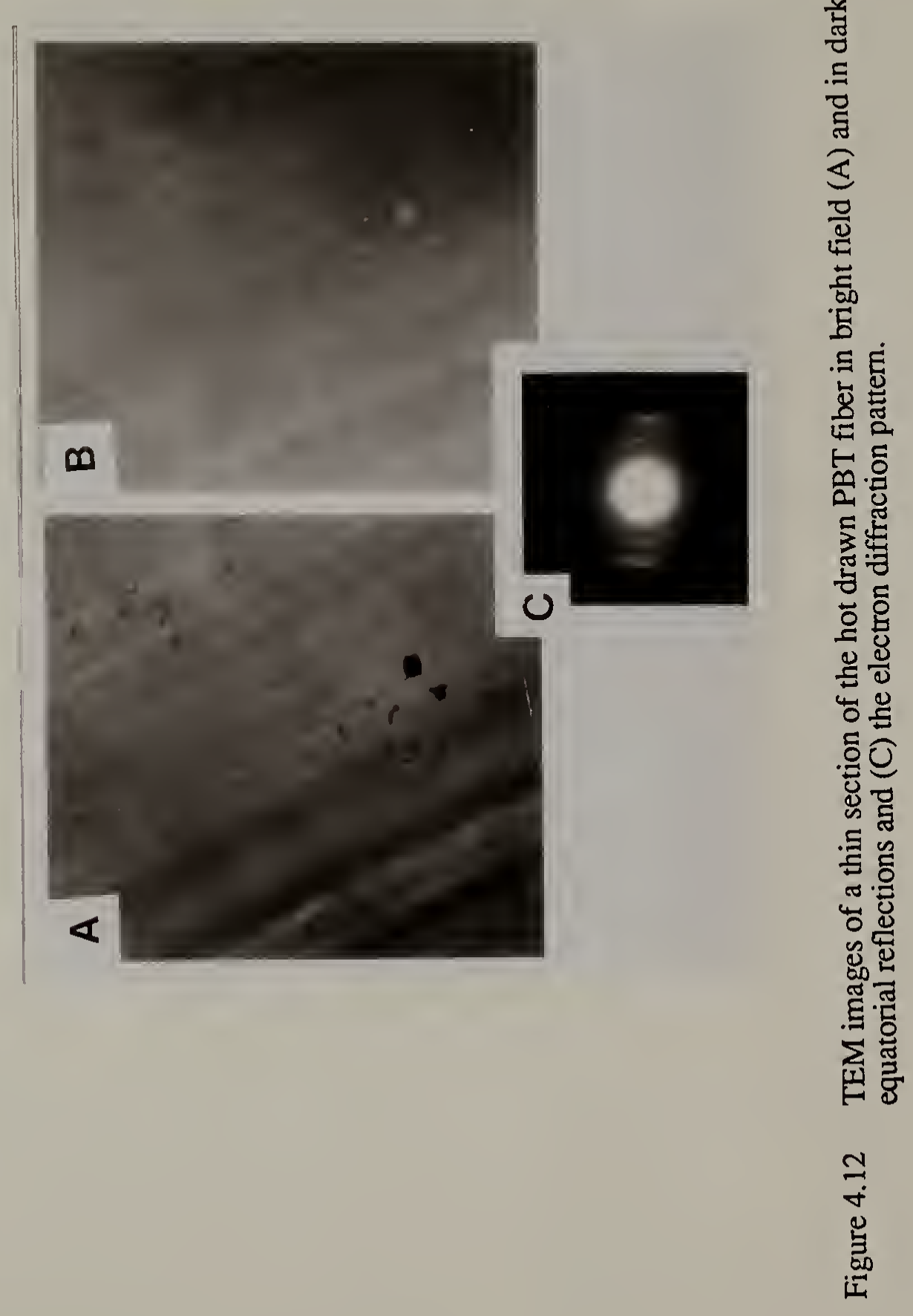




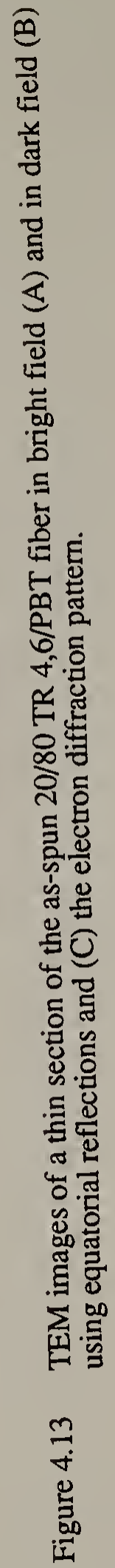




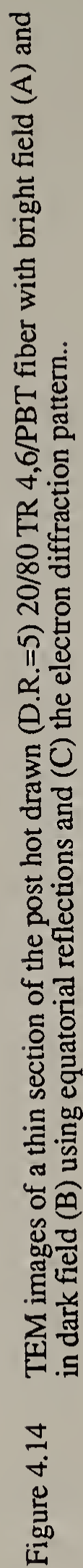




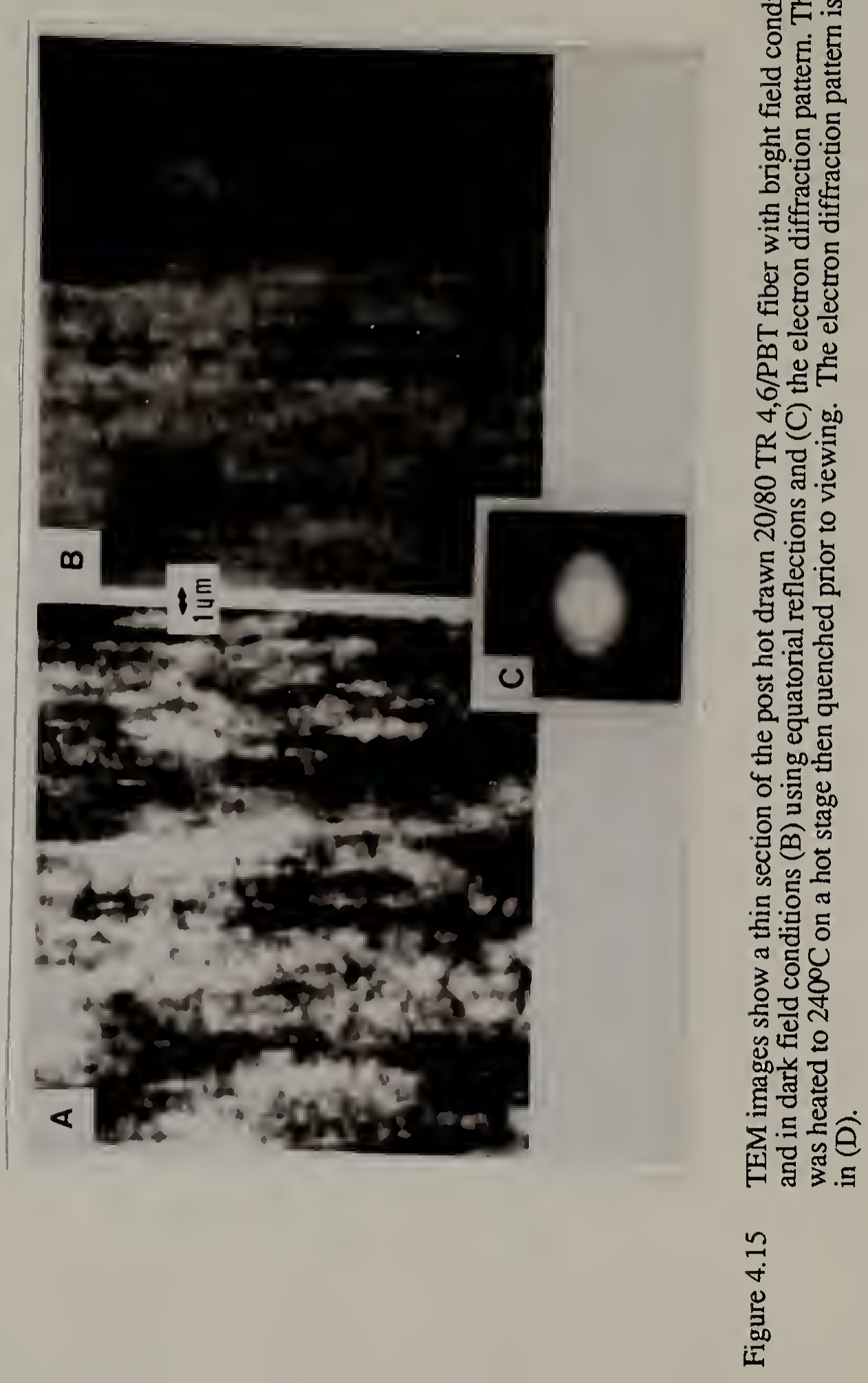

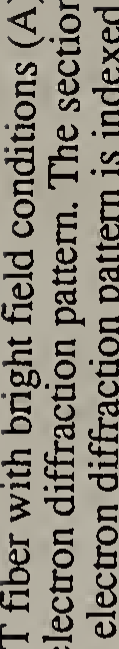

0

OO

政

.

옹

岛. 可

․ㅠㅁํㅇ

웜

正

य 다

o.

o.

蛋

论

ธ

릉

ธบ

응요

的

임

हี శึ

$\Sigma . \Xi \pm$

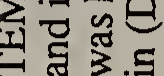

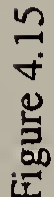


Ajji, A., Brisson, J. and Qu, Y., Journal of Polymer Science: Part B: Polymer Physics 30 , pp. 505-516(1992). "Orientation and Mechanical Properties of PBT and Its Blends with Liquid-Crystalline Copolyester".

Bassett, B. R. and Yee, A. F., Polymer Composites, 11, pp. 10-18(1990). "A Method of Forming Composite Structures Using In Situ-Formed Liquid Crystal Polymer Fibers in a Thermoplastic Matrix".

Beery, D., Kenig, S. and Siegmann, A., Polymer Engineering and Science, 31, pp. 451458(1991). "Structure Development During Flow of Polyblends Containing Liquid Crystalline Polymers".

Beery, D., Kenig, S. and Siegmann, A., Polymer Engineering and Science, 31, pp. 459 466(1991). "Structure and Properties of Molded Polyblends Containing Liquid Crystalline Polymers".

Delimoy, D.; Bailly, Christian; Devaux, J.; and Legras, R.; Polymer Engineering and Science, 28, pp. 104-112(1988). "Morphological Studies of PolycarbonatePolybutylene Terephthalate) Blends by Transmission Electron Microscopy".

De Teresa, S. J., Allen, S. R., Farris, R. J. and Porter, R. S., Journal of Materials Science, 19 , pp. 57(1984). "Compressive and Torsional Properties of Kevlar 49 Fibers".

Hwang, C. R., PhD. Dissertation, Polymer Science and Engineering Department, University of Massachusetts (1989). "Molecular' and 'In-situ Network' Composites of Poly(p-Phenylene benzobisthiazole) and Nylons".

Kenig, S., Polymer Engineering and Science, 27, pp. 887-892(1987). "Orientability of Liquid Crystal Polymers in Elongational Flow".

Kenig, S., Polymer Engineering and Science, 29, pp. 1136-1141(1989). "Orientability of Liquid Crystal Polymers in Elongational Flow".

Koningsveld, R.; Pure and Applied Chemistry, 61, pp. 1051-1064(1989).

Krause S. J., Haddock, T. , Price, G. E., Lengert, P. G., O'Brien, J. F., Helminiak, Thaddeus E., Adams, W. W., Journal of Polymer Science: Part B: Polymer Physics, 24,1991,(1986). "Morphology of a Phase-Separated and a Molecular Composite PBT/ABPBI Polymer Blend".

Ludwig, H.-J. and Eyerer, P., Polymer Engineering and Science 29, pp. 143-146(1988). "Influence of Processing Conditions on Morphology and Deformation Behavior of Poly(Butylene Terephthalate) (PBT)".

Misra, A. and Stein, R. S., Journal of Polymer Science: Part B: Polymer Physics, 18, pp.1145-1158(1980). "Morphological Studies on Poly(butylene terephthalate)". 
Pottick, L. A., PhD. Dissertation, Polymer Science and Engineering Department, University of Massachusetts (1986). "The Influence of Drying on the Structure and Mechanics of Poly(p-Phenylene Benzobisthiazole) Fibers".

Roche, E. J., Stein, R. S. and Thomas, E. L. Journal of Polymer Science: Part B: Polymer Physics, 18, pp.1145-1158(1980). "Electron Microscopy of the Structure of Normal and Abnormal Poly(butylene Terephthalate)".

Yeh, J. T. and Runt, J. . Journal of Polymer Science: Part B: Polymer Physics, 27, pp. 1543-1550(1989). "Multiple Melting in Annealed Poly(butylene Terephthalate)". 


\section{BIBLIOGRAPHY}

Ajji, A., Brisson, J. and Qu, Y., Journal of Polymer Science: Part B: Polymer Physics, $\underline{30}$, pp. 505-516(1992). "Orientation and Mechanical Properties of PBT and Its Blends with Liquid-Crystalline Copolyester".

Alexander, L. E., X-Ray Diffraction Methods in Polymer Science, Robert E. Krieger Publishing Co., Malabar, FL, 1985.

Allen, S. R., Journal of Materials Science, 22, pp. 853-859(1987). "Tensile recoil measurement of compressive strength for polymeric high performance".

Allen, S. R., Ph. D. Thesis, University of Massachusetts, Amherst, MA (1983). "Mechanical and Morphological Correlations in Poly(p-phenylene benzobisthiazole) Fibers".

Allen, S. R., Polymer, 29, pp. 1091-1094(1989). "Stress-coupling phenomena in anisotropic fibres".

Argon, A. S., Treatise on Materials Sciences and Technology, Volume 1, Academic Press, New York, (1972). "Fracture of Composites".

Ballauff, M., Journal of Polymer Science, Polymer Physics Edition, 25, pp. 739747(1987). "Compatibility of Coils and Rods Bearing Flexible Side Chains".

Ballauff, M., Molecular Crystals Liquid Crystals, 168, pp. 209-228(1989). "The Floury Lattice Model of Nematic Fluids".

Barbero, G. and Oldano, C., Molecular Crystals Liquid Crystals, 168, pp. 1-5(1989). "On the $\mathrm{K}_{13}$-Dependent Energy Term in Nematic Liquid Crystals".

Bassett, B. R. and Yee, A. F., Polymer Composites, 11, pp. 10-18(1990). "A Method of Forming Composite Structures Using In Situ-Formed Liquid Crystal Polymer Fibers in a Thermoplastic Matrix".

Beery, D., Kenig, S. and Siegmann, A., Polymer Engineering and Science, 31, pp. 451458(1991). "Structure Development During Flow of Polyblends Containing Liquid Crystalline Polymers".

Beery, D., Kenig, S. and Siegmann, A., Polymer Engineering and Science, 31, pp. 459466(1991). "Structure and Properties of Molded Polyblends Containing Liquid Crystalline Polymers".

Blundell, D. J.; Crick, R. A.; Fife, B.; Peacock, J.; Keller, A.; Waddon, A.; Journal of Materials Science, 24,2057-2064(1989). "Spherulitic Morphology of the Matrix of Thermoplastic PEEK/Carbon Fibre Aromatic Polymer Composites".

Boehm, R. E., Martire, D. E., and Madhusudana, N. V., Macromolecules, 19, pp. 23292341 (1986). "A Statistical Thermodynamic Theory of Thermotropic Linear Main-Chain Polymeric Liquid Crystals". 
Chandrasekhar, S., Liquid Crystals, Cambridge University Press, Cambrigde, 1977. Clark, M. G., Molecular $\frac{\text { Crystals Liquid }}{\text { Crystals, }}$ 127, pp. 1-41(1985). "Macroscopic
Properties of Liquid Crystals".

Cohen, Y. and Thomas, E. L., Macromolecules, 21, 433, (1988) "Microfibrillar Network of a Rigid Rod Polymer. 1. Visualization by Electron Microscopy".

Cohen, Y., Farris, R. J., and De Teresa, S. J., U.S. Patent Number 899910, 25 August 1986. "Novel Composites based on Reinforcement with Microfibrillar Networks of Rigid-Rod Polymers".

Cohen, Y., Farris, R. J., and De Teresa, S. J., U.S. Patent Number 4842924, 27 June 1989. "Novel Composites based on Reinforcement with Microfibrillar Networks of Rigid-Rod Polymers".

Cohen, Y., Farris, R. J., and De Teresa, S. J., U.S. Patent Number 5102601, 7 April 1992. "Process for Fabricating Novel Composites Based on Reinforcement with Microfibrillar Networks of Rigid-Rod Polymers".

Cohen, Y., Farris, R. J., and De Teresa, S. J., United Kingdom Patent 2195672, 14 August 1990. "Novel Composites based on Reinforcement with Microfibrillar Networks of Rigid-Rod Polymers".

Cohen, Y., "Structure Formation in Solutions of Rigid Polymers Undergoing Phase Transitions", AFWAL-TR-87-4030, AFWAL/MLBP, Wright Patterson Airforce Base, April, 1987.

Cotter, M. A., Phil. Trans. R. Soc. Lond, A309, pp. 127-144 (1983) "The Van der Waals Theory of Nematic Liquids".

Croxton, C., Molecular Crystals Liquid Crystals, 59, pp.219-240(1980). "Statistical Thermodynamics of the Liquid Crystal Surface, Smectic, Nematic and Isotropic System".

Dawson, P. C., Blundell, D. J., Polymer, 21, 578, (1980). "X-ray data for poly(arylether ketones).

Delimoy, D.; Bailly, Christian; Devaux, J.; and Legras, R.; Polymer Engineering and Science, 28, pp. 104-112(1988). "Morphological Studies of PolycarbonatePolybutylene Terephthalate) Blends by Transmission Electron Microscopy".

De Teresa, S. J., Allen, S. R., Farris, R. J. and Porter, R. S., Journal of Materials Science, 19 , pp. 57(1984). "Compressive and Torsional Properties of Kevlar 49 Fibers".

De Teresa, S. J., Ph. D. Thesis, University of Massachusetts, Amherst (1986). "Axial Compressive Strength of High Performance Polymer Fibers".

Doi, M. and Edwards, F. S., Theory of Polymer Dynamics, Claredon Press, Oxford, 1986. 
Drzaic, P., Molecular Crystals Liquid Crystals, 154, pp. 289-306(1988). "A New Director Alignment for Droplets of Nematic Liquid Crystal with Low Bend to Splay Ratio".

Farris, R. J.; Malone, M. F.; Schaffer, K. R.; "Morphological Studies of PBZT/PEKK Composite Fibers," October 1989 Report to E. I. du Pont de Nemours, Inc.

Fieser, R. G. and Goldsmith, C. C., Glass Technology, 19, pp. 10(1978). "Devitrification studies of Corning 7575 seal glass".

Flory, P. J. and Ranch, G., Molecular Crystals Liquid Crystals 54, 289-310(1979). "Theory of Systems of Rodlike Particles, I. Athermal systems".

Flory, P. J. and Ronca, G., Molecular Crystals Liquid Crystals 54, pp. 31 1-330(1979). "Theory of Systems of Rodlike Particles".

Flory, P. J., Macromolecules, 11, pp. 1138-1141(1978). "Statistical Thermodynamics of Mixtures of Rodlike Particles. 5. Mixtures with Random Coils".

Flory, P. J., Proceedings of the Royal Society, A, 234, pp. 73-89(1956). "Phase Equilibria in Solutions of Rod-like Particles".

Frank , F. C., Discussions of the Faraday Society, $\underline{25}$, pp. 19-28(1958). "On the Theory of Liquid Crystals".

Frederico, O., Ph. D. Dissertation, Polymer Science and Engineering Department, University of Massachusetts, Amherst, MA (1989). "In-situ Composites of a Thermotropic Liquid Crystalline Polymer and Polycarbonate: Processing, Morphology and Properties".

Gabriel, C. A., PhD. Dissertation, Polymer Science and Engineering Department, University of Massachusetts (1987). "Processing and Properties of Composite Fibers of Poly (p-phenylene Benzobisthiazole) and Poly(ether ether ketone) or Nylon 6,6".

Gennes, P. G. de, Liquid Crystals and Plastic Crystals, Volume 1, Ellis Horwood Limited, Chichester, (1974), G. W. Gray and P. A. Winsor, editors. "Principles of Continuum Theory".

Gennes, P. G. de, The Physics of Liquid Crystals, Clarendon Press, Oxford(1974).

Goosens, W. J. A., Molecular Crystals Liquid Crystals, 124, pp. 305-331(1985). "Bulk, Interfacial and Anchoring Energies of Liquid ".

Hedmark, P. G., Lopez, J. M. R., Westdahl, M., Werner, P., Jansson, J., and Gedde, U. W., Polymer Engineering and Science, 28(19), pp. 1248-1259(1988).

"Microstructure in Injection Molded Samples of Liquid Crystalline Poly(PHydroxy-Benzoic Acid-Co-Ethylene Terephthalate)".

Hermans, J. J., Hermans, P. H., Vermaas, D., and Weidinger, A., Rec. trav. chim. Paysbas, $\underline{65}$, pp. 427 427(1946). "Quantitative Evaluation of Orientation in Cellulose Fibres from X-ray Fibre Diagram". 
Hindeleh, A. M. and Johnson, D. J., Polymer, 1980, Volume 21, page 929. "An Empirical Estimation of Scherrer Parameters for the Evaluation of True Crystallite Size in Fibrous Polymers".

Hinov, H. P., Molecular Crystals Liquid Crystals, 168, pp. 7-12(1989). "Comment on the Criticism of the One-Dimensional Solution of the $\mathrm{K}_{13}$ Elastic Problems in Nematics".

Hwang, C. R., Malone, M. F. and Farris, R. J., Journal of Materials Science, 26, pp. 1762-1768(1991). "Molecular composites of poly(p-phenylene benzobisthizole) with thermoplastics: coagulation studies".

Hwang, C. R., Malone, M. F., Farris, R. J., Martin, D. C. and Thomas, E. L., Journal of Materials Science, 26, pp. 1768-1772(1991). "Microstructure and mechanical properties of in-situ network composite fibres of PBZT with nylon".

Hwang, C. R., PhD. Dissertation, Polymer Science and Engineering Department, University of Massachusetts (1989). "Molecular' and 'In-situ Network' Composites of Poly(p-Phenylene benzobisthiazole) and Nylons".

Hwang, W-F.; Wiff, D. R.; Verschoore, C., Price, G. E.; Helminiak, T. E.: Adams, W. W.; Polymer Engineering and Science, 23, pp. 784-788 (1983). "Solution Processing and Properties of Molecular Compostie Fibers and Films".

Katsaros, J. D., Malone, M. F., and Winter, H. H., Polymer Engineering and Science, 29, pp. 1434-1445(1989. "The Effects of Flow on Miscibility in a Blend of Polystyrene and Poly(Vinyl Methyl Ether)".

Kenig, S., Polymer Engineering and Science, 27, pp. 887-892(1987). "Orientability of Liquid Crystal Polymers in Elongational Flow".

Kenig, S., Polymer Engineering and Science, 29, pp. 1136-1141(1989). "Orientability of Liquid Crystal Polymers in Elongational Flow".

Kle'man, M., Points, Lines and Walls, John Wiley and Sons, New York, 1983.

Koningsveld, R.; Pure and Applied Chemistry, 61, pp. 1051-1064(1989). "Thermodynamics of Macromolecule Systems"

Kovar, R. F. and Lusignea, R. W., AFB 0097-FM- 8535-41, Air Force Office of Scientific Research, March, 1986. "Microcomposite Processing and Applications".

Kovar, R. F., AFB-0022.FM-8689-3, Airforce Office of Scientific Research, August, 1987. "Microcomposite Processing and Applications".

Kovar, R. F.; Haghighat, R.; and Lusignea, R. W.; Materials Research Society Symposium Proceedings, Vol. 134, (1989). "Improvement of Compressive Strength in Ordered Polymer Films and Fibers by Sol-Gel Processing". 
Krause S. J., Haddock, T. , Price, G. E., Lengert, P. G., O'Brien, J. F., Helminiak, Thaddeus E. , Adams, W. W., Journal of Polymer Science: Part B: Polymer Physics, 24,1991,(1986). "Morphology of a Phase-Separated and a Molecular Composite PBT/ABPBI Polymer Blend".

Krause S. J., Haddock, T. B.,Vezie,D. L., Lengert, P. G.,Hwang, W. F., Price, G E., Helminiak, T. E. , O'Brien, J F., Adams, W. W., Polymer, 29(1988).

"Morphology and properties of rigid-rod poly(p-phenylene benzobisoxazole) (PBO) and stiff-chain poly(2,5(6)-benzoxazole)(ABPBO) fibers".

Krough-Moe, J. and Wold-Hansen, P. S., Acta Cryst., B29, pp. 2242-2246 (1973).

"The Crystal Structure of Hexalead Pentaborate, $6 \mathrm{PbO} .5 \mathrm{~B}_{2} \mathrm{O}_{3}$ ".

Kumar, Satish and Wang, C. S., Polymer Communications, 29, pp. 355-356(1988). "Xray Study of PBT/nylon 66 Composite".

La Mantia, F. P.; Valenza, A.; Paci, M.; and Magagnini, P. L., Polymer Engineering and Science, 30, pp. 7-12(1990). "Rheology-Morphology Relationships in Nylon 6/Liquid-Crystalline Polymer Blends".

Lee, Sin-Doo and Meyer, Robert B., Physical Review Letters, 61, pp. 2217-2220. "Crossover Behavior of the Elastic Coefficients and Viscosities of a Polymer Nematic Liquid Crystal".

Lovinger, Andrew J. and Davis, D. D., Macromolecules, 19, pp. 1861-1867(1986).

"Solution Crystallization of Poly(ether ether ketone)".

Luckhurst, G. R., Nuclear Magnetic Resonance of Liquid Crystals, edited by J. W. Emley, pp. 85-97, NATO ASI Series, Series C: Mathematical and Physical Sciences Volume 141., 1983, D. Reidel Publishing Company, Dordrecht, Holland. "The Landau-de Gennes Theory of Liquid Crystals".

Luckhurst, G. R.; Nuclear Magnetic Resonance of Liquid Crystals, edited by J. W. Emley, pp. 53-83, NATO ASI Series, Series C: Mathematical and Physical Sciences Volume 141., 1983, D. Reidel Publishing Company, Dordrecht, Holland. "The Molecular Field Theories of Nematics: Systems Composed of Uniaxial, Biaxial or Flexible Molecules".

Ludwig, H.-J. and Eyerer, P., Polymer Engineering and Science, 29, pp. 143-146(1988). "Influence of Processing Conditions on Morphology and Deformation Behavior of Poly(Butylene Terephthalate) (PBT)".

Lusignea, Richard W., Materials Research Society Symposium Proceedings, Vol. 134, (1989).

Magliochetti, M., PhD. Dissertation, Polymer Science and Engineering Department, University of Massachusetts (1990). "Solution Viscosity and Phase Behavior of Lyotropic Liquid Crystalline and Flexible Polymers".

Matheson, R. R. and Flory, P. J., Macromolecules, 14, pp. 954-960(1981). "Statistical thermodynamics of Mixtures of Semirigid Macromolecules: Chains with Rodlike Sequences at Fixed Locations". 
McMurdie, Howard F. and Elmer N. Bunting, Journal of the National Bureau of $\frac{\text { Standards, }}{\mathrm{PbO}-\mathrm{SiO}_{2}}$ "

Meyer, Robert B., Molecular Fluids, Gordon and Breach Science Publishers, London, R. Balian and G. Weill,(1973) editors, pp. 271-343. "Structural Problems in
Liquid Crystal Physics".

Meyer, Robert B., Polymer Liquid Crystals, Academic Press, New York, 1982, A. Ciferri, W. R. Krigbaum, and R. B. Meyer, editors. Chapter 6, "Macroscopic Phenomenon in Nematic Polymers".

Minter,J. R., Structural Investigation of Fibers and Films of Poly(pphenylenebenzobisthiazole), AFWAL-TR-82.4097, Vol I, Materials Laboratory (AFWAK/MLBP), May 1982.

Misra, A. and Stein, R. S., Journal of Polymer Science: Part B: Polymer Physics, $\underline{18}$, pp.1145-1158(1980). "Morphological Studies on Poly(butylene terephthalate)".

Nehring, J., Saupe, A., Journal of Chemical Physics, 54, pp. 337-343(1971). "On the Elastic Theory of Uniaxial Liquid Crystals".

Nehring, J., Saupe, A., Journal of Chemical Physics, 56, pp. 5527-5528(1972).

"Calculation of the Elastic Constants of Nematic Liquid Crystals".

Newman, K. E., Zhang, P., Cuddy, L. J., and, Allara, D. L.; Journal of Materials $\underline{\text { Research }} \underline{6}$, pp. 1580-1594 (1991).

Ottino, J., The Kinematics of Mixing: Stretching, Chaos, and Transport, Cambridge University Press, Cambridge 1989.

Perloff, A., and Block, S., Acta Cryst., 20, pp. 274 (1966). "The Crystal Structure of the Strontium and Lead Tetraborates, $\mathrm{SrO} .2 \mathrm{~B}_{2} \mathrm{O}_{5}$ and $\mathrm{PbO} .2 \mathrm{~B}_{2} \mathrm{O}_{3}$ ".

Perusich, Renee, unpublished data presented February 14, 1990 for U.S. Air Force.

Pottick, L. A., PhD. Dissertation, Polymer Science and Engineering Department, University of Massachusetts (1986). "The Influence of Drying on the Structure and Mechanics of Poly(p-Phenylene Benzobisthiazole) Fibers".

Priest, R. G., Physical Review A, 7, pp. 720-729(1973). "Theory of the Frank Elastic Constants of Nematic Liquid Crystals".

Roche, E. J., Stein, R. S. and Thomas, E. L. Journal of Polymer Science: Part B: Polymer Physics, 18 , pp.1145-1158(1980). "Electron Microscopy of the Structure of Normal and Abnormal Poly(butylene Terephthalate)".

Rueda, D. R.; Ania, F.; Richarson A.; Ward I. M.; Balta, Calleja, F. J.; Polymer Communications, 24 pp. 258(1983), "X-ray Diffraction Study of Die Drawn Poly(aryletherketone) (PEEK)". 
Samulski, E. T., Polymeric Liquid Crystals, Plenum Press, New York and London 1985, Alexandre Blumstein, editor. "Some Physico-Chemical Aspects of Polymeric
Liquid Crystals".

Straley, J. P., Physical Review A, $\underline{8}$, pp. 2181-2183(1973). "Frank Elastic of the HardRod Liquid Crystals".

Takayanagi Motowo, Interrelations Between Processing Structure and Properties of Polymeric Materials, pp. 43-56, J. C. Seferis and P. S. Theocaris, editors, Elsevier Science Publishers B. V. Amsterdam, The Netherlands (1984), "Polymer Composites of Rigid and Flexible Molecules".

Telo da Gama, M. M., Molecular Physics, 52, pp. -611-630 (1984). "The Interfacial Properties of a Model of a Nematic Liquid Crystal II. Induced Orientational Order and Wetting Transitions at a Solid-Fluid Interface ".

Telo de Gama, M. M., Molecular Physics, 52, pp. 585-610 (1984). "The Interfacial Properties of a Model of a Nematic Liquid Crystal I. The Nematic-Isotropic and the Nematic-Vapor Interfaces".

Thomas, E. L. and Wood, B. A., Faraday Discussions of the Chemical Society 79, pp. 229-239 (1985). "Mesophase Texture and Defects in Thermotropic LiquidCrystalline Polymers".

Vertogen, G., de Jeu, W. H., Thermotropic Liquid Crystals, Fundamentals, SpringerVerlag, Berlin, 1985.

Warner, M.; Molecular Physics, 52, 677-690(1984). "Fluctuations, Mean Fields and the Order Parameters in Nematics".

Wood, B. A., Ph. D. Dissertation, Polymer Science and Engineering Department, University of Massachusetts, Amherst, MA (1985). "Ultrastructural Studies of Thermotropic Liquid Crystalline Polymers".

Wycliffe, S. M., Malone, M.F. and Farris, R.J., Journal of Applied Polymer Science, 35 , pp. 1955-1962(1987). "Processing and Properties of Composite Fibers of Poly (p-phenylene Benzobisthiazole)/ Nylon Fibers".

Yeh, J. T. and Runt, J. , Journal of Polymer Science: Part B: Polymer Physics, 27, pp. 1543-1550(1989). "Multiple Melting in Annealed Poly(butylene Terephthalate)".

Yokoyama, H., Molecular Crystals Liquid Crystals, 165 , pp. 265-316(1988). "Surface Anchoring of Nematic Liquid Crystals". 
